\title{
DETERMINAÇÃO DE RIGIDEZ DE ESTRUTURAS DE PAVIMENTOS ATRAVÉS DOS MÉTODOS DOS ELEMENTOS DE CONTORNO E FINITOS
}

\section{ALUNA: REGINA MARIA DOS SANTOS CARMO}

Tese apresentada à Escola de Engenharia de São Carlos, da Universidade de São Paulo, como parte dos requisitos para obtenção do título de Doutor em Engenharia de Estruturas.

ORIENTADOR: WILSON SÉRGIO VENTURINI

São Carlos 
Aos meus pais, irmãs e irmãos Com carinho. 


\section{AGRADECIMENTOS}

Em primeiro lugar, gostaria de agradecer profundamente ao Professor Dr. Wilson Sérgio Venturini, pelo trabalho de orientação e por todas as grandes oportunidades que me deu. Agradeço, também pela amizade e acima de tudo pela paciência nos momentos mais difíceis.

Agradeço, particular e especialmente aos meus pais e irmãos pela orientação espiritual e emocional e pelos conselhos sempre úteis.

Aos Professores Drs. Nelson K. Salgado (ITA), Humberto Breves Coda (EESC-USP), Fernando Amorim de Paula (UFMG) e respectivas famílias, por terem sido companheiros e orientadores em todos os momentos durante o estágio em Southampton-UK.

Ao Professor Dr. M.H. Aliabadi, pela orientação durante estágio no Wessex Institute of Technology, UK.

A todos os meus amigos, colegas pela amizade.

Aos funcionários do Departamento de Engenharia de Estruturas e da EESC pelos anos de convívio e excelentes serviços prestados. 


\section{SUMÁRIO}

CAPÍTULO 1 - INTRODUÇÃO 1

1.1 - OBJETIVO 5

1.2 - APRESENTAÇÃO POR CAPÍTULOS 6

CAPÍTULO 2 - TEORIA DE KIRCHHOFF PARA PLACAS DELGADAS 8

2.1 - INTRODUÇÃO 8

2.2 - ANÁLISE DE PLACAS FINAS PELA TEORIA DE KIRCHHOFF 8

$\begin{array}{ll}2.3 \text { - DESLOCAMENTOS } & 10\end{array}$

2.4 - DEFORMAÇÕES 11

2.5 - TENSÕES 12

2.6 - COMPONENTES ESFORÇOS 12

2.7 - EQUAÇÃO DIFERENCIAL DE PLACAS 13

2.8 - VALORES DE CONTORNO 14

2.8 - CORTANTE EQUIVALENTE 15

2.9 - FORÇAS CONCENTRADAS NOS CANTOS 16

2.10 - SOLUÇÃO FUNDAMENTAL 16

2.11 - REPRESENTAÇÃO INTEGRAL PARA PONTOS DO DOMÍNIO 19

2.12 - REPRESENTAÇÃO INTEGRAL PARA PONTOS DO ONTORNO 23

2.13 - INTEGRAIS DE DOMÍNIO 26

2.13.1 - CARGAS DISTRIBUÍDAS EM REGIÕES DA PLACA 26

2.13.2 - CARGAS DISTRIBUÍDAS EM LINHAS OU CARGAS 28

EM REGIÕES DISCRETAS

2.14 - O MEC APLICADO À ANÁLISE DE PLACAS DELGADAS 29

2.15 - INTEGRAÇÃO SOBRE OS ELEMENTOS 39

2.16.1 - INTEGRAIS NUMÉRICAS 41

2.16.2 - INTEGRAIS ANALÍTICAS 41

2.16.3 - INTEGRAIS NUMÉRICAS SUBELEMENTADAS 42 
CAPÍTULO 3 - MÉTODO DOS ELEMENTOS DE CONTORNO APLICADO À ANÁLISE DE CHAPAS

3.1 - INTRODUÇÃO 44

3.2 - HIPÓTESES BÁSICAS 45

3.3 - ESTADO DE TENSÕES 47

3.4 - ESTADO DE DEFORMAÇÕES 48

3.5 - RELAÇÕES CONSTITUTIVAS

3.6 - EQUAÇÃO DIFERENCIAL PARA O EPT (NAVIER) 50

3.7 - SOLUÇÃO FUNDAMENTAL 50

3.8 - REPRESENTAÇÃO INTEGRAL PARA PONTOS DO 52 DOMÍNIO

3.9 - REPRESENTAÇÃO INTEGRAL PARA PONTOS DO 53 CONTORNO

3.10 - O MEC APLICADO À ANÁLISE DE CHAPAS SOB EPT

CAPÍTULO 4 - ELEMENTO DE BARRA MODELADO PELO MÉTODO DOS ELEMENTOS FINITOS

4.1 - INTRODUÇÃO

61

4.2 - MATRIZ DE TRANSFORMAÇÃO DO VETOR DE FORÇA PARA

VIABILIZAR O ACOPLAMENTO

4.2.1 - A MATRIZ $\underline{\mathrm{C}}$ 63

4.2.2 - ORIENTAÇÃO DAS BARRAS - MATRIZ DE

TRANSFORMAÇÃO DE COORDENADAS

\section{CAPÍTULO 5 - TÉCNICA DAS SUBREGIÕES}

5.1 - INTRODUÇÃO 76

5.2 - FORMULAÇÃO BÁSICA SENTIDOS POSITIVOS 77

5.3 - SUB-REGIÕES EM PLACAS DE KIRCHHFF 83

5.4 - SUB-REGIÕES ACOPLADAS NUMA INTERFACE COM 83 INFLUÊNCIA EXTERNA

5.5 - MAIS DE DUAS SUB-REGIÕES ACOPLADAS NUMA MESMA INTERFACE

5.5 - UM PROCEDIMENTO ALTERNATIVO PARA A 90 CONSIDERAÇÃO DE SUBDIVISÃO DO DOMÍNIO 
5.6 - EXEMPLOS 92

5.6.1 - EXEMPLOS I, II E III 92

5.6.2 - EXEMPLO IV 95

CAPÍTULO 6 - COMBINAÇÃO MEC/MEF 98

6.1- INTRODUÇÃO 98

6.2 - COORDENADAS GLOBAIS DOS ELEMENTOS ESTRUTURAIS 100

6.3 - COORDENADAS DOS ELEMENTOS DE PLACA 101

6.3.1 - ESFORÇOS EM UM PONTO DA PLACA 101

6.3.2 - DESLOCAMENTOS EM UM PONTO DA PLACA 102

6.3.3 - TRANSFORMAÇÃO ENTRE COORDENADAS LOCAIS E 103

GLOBAIS PARA NN PONTOS DA PLACA

6.4 - COORDENADAS DOS ELEMENTOS DE BARRAS 104

6.5 - CONDIÇÕES DE CONTORNO DA COMBINAÇÃO 105

6.5.1 - CONDIÇÕES DE CONTORNO NO MEF 106

6.5.1.1 - NÓS NÃO-LIGADOS 106

6.5.1.2 - NÓS DE INTERFACE SEM INFLUÊNCIA 107

EXTERNA

6.5.1.3 - NÓS DE INTERFACE COM INFLUÊNCIA 108

EXTERNA

6.5.1.4 - NÓS DA BARRA LIGADOS A NÓS 109

INTERNOS DA PLACA

6.6 - EXEMPLOS 112

6.7 - EFEITO DE MEMBRANA - ESTADO PLANO DE TENSÃO 114

6.7.1 - COMBINAÇÃO DOS ELEMENTOS PLACA E CHAPA 116

6.7.2 - IDEALIZAÇÃO DO MODELO A PARTIR DO PTV 119

6.7.2.1-LIGAÇÃO ENTRE REGIÕES DE PLACAS 121

(MEC/MEC)

6.7.2.2 - LIGAÇÃO ENTRE REGIÕES DE PLACAS 129

E BARRAS (MEC/MEF)

6.7.2.3 - EXEMPLOS

132 


\section{POR SUBESTRUTURAÇÃO}

7.1 - INTRODUÇÃO 6.1- INTRODUÇÃO

7.2 - SOLUÇÃO DIRETA UTILIZANDO-SE ALGORITMO

BASEADO NA ELIMINAÇÃO DE GAUSS

7.3 - ANÁLISE POR SUBESTRUTURAÇÃO

139

7.4 - MÉTODO DA CONDENSAÇÃO ESTÁTICA PARA O SISTEMA

MODELADO PELA COMBINAÇÃO DO MEC COM O MEF 


\section{RESUMO}

O tema desta pesquisa refere-se ao tratamento numérico de estruturas de pavimentos, dando ênfase à utilização do método dos elementos de contorno - MEC para o tratamento de elementos planos, placas, enquanto os elementos lineares serão tratados através do método dos elementos finitos - MEF. Busca-se contribuir com uma série de trabalhos realizados nesta área, principalmente com a consideração do efeito da excentricidade do eixo neutro das barras em relação à superfície neutra da placa somando-o, portanto, ao fenômeno de flexão desta última. A técnica de acoplamento dos elementos estruturais utilizada foi a técnica das sub-regiões, que facilita a visualização do problema da combinação e viabiliza o uso da técnica de condensação estática na resolução do sistema de equações.

Palavras-chave: Método dos Elementos de Contorno; Análise de Placas Delgadas; Análise de Pavimentos; Combinação MEC/MEF. 


\section{ABSTRACT}

This research refers to the numeric analysis of structures, emphasizing the use of the boundary element method- BEM- in the discretization of the plates, while the linear elements will be formulated through the finite element method - FEM. The aim is to contribute with a number of works related to this subject, specially in the consideration of the effect of the stiffeners' eccentricity in relation to the plates neutral surface, in addition to its bending state. The tool adopted to fulfill the coupling among these kinds of structural elements is the well-known sub region or multi-domain technique, which works well with the static condensation technique for solving the sparse system of equation generated.

Keywords: Boundary Element Method; Thin Plates Bending Analysis; Structural Analysis of Buildings; BEM/FEM Coupling. 


\section{CAPÍTULO 1 - INTRODUÇÃO}

Neste trabalho desenvolveu-se um estudo da formulação do Método dos Elementos de Contorno (MEC) para a análise de placas finas, com base nas aproximações de Kirchhoff, associadas a elementos de barra modelados pelo Método dos Elementos Finitos (MEF). As barras podem estar dispostas seja no domínio seja no contorno das placas. O efeito da excentricidade do eixo neutro das barras em relação à superfície neutra da placa é considerado somando-se, portanto, ao fenômeno de flexão da mesma, um estado plano de tensões (EPT).

Este acoplamento foi feito considerando-se a associação espacial de subregiões que agrupam elementos de mesmas propriedades físicas e materiais. O sistema final de equações é constituído de acordo com o tratamento individual de cada elemento ou grupo de elementos estruturais (placas de mesma propriedades físicas e geométricas e estruturas constituídas por barras) como um subdomínio do pavimento. A compatibilização final é feita após uma devida transformação das coordenadas de cada sub-região. Esta técnica facilita a visualização do conjunto como um todo e também a viabilidade de combinações entre os métodos. 
Trabalhos como os de WEARING \& BETTAHAR (1994) discutem o uso da técnica da subdivisão do domínio da placa no MEC para a análise de sua flexão. Avalia-se também a eficiência da formulação proposta para algumas variações da geometria das placas. Como contribuição aos estudos da técnica de sub-regiões aplicada ao MEC, VENTURINI (1989) e VENTURINI \& PAIVA (1988) estabelecem avanço na formulação do elemento de contorno considerando o domínio de uma placa sob flexão subdividido, sem a separação física ao longo das interfaces. elaboram esta formulação para análise de placas sob flexão divididas em regiões de diferentes propriedades e geometria. Eliminam, desta forma, aproximações internas. Este trabalho gerou uma série de outros trabalhos como o de CHAVES et al. (1999) e mais recentemente FERNANDES, CODA \& VENTURINI (2000) que elaboraram uma formulação para combinar placas e barras, ambos modelados pelo MEC, sem que se necessite a construção das matrizes de influência de cada subregião separadamente.

Os primeiros trabalhos que trataram placas no contexto de estruturas de pisos de edifícios através do MEC, embora não especificados no texto, foram os de BÉZINE (1981), e HARTMANN \& ZOTEMANTEL (1986) e GUO-SHU (1986). No primeiro, o autor desenvolveu um algoritmo onde condições de domínio podem ser impostas, dando assim o primeiro passo para a simulação de apoios de lajes de edifícios (lajes contínuas). Nos dois outros trabalhos referenciados, os autores também possibilitaram a colocação de vínculos internos inclusive elásticos. Mais recentemente, HARTLEY(1996) apresenta a versatilidade do uso do MEC para a análise do comportamento da placa interconectada a outros elementos de estruturas complexas de edifícios. Enfatiza as aproximações adotadas para facilitar a elaboração da formulação, quando transfere para a placa a influência das barras sobre o seu comportamento estrutural.

Inúmeras pesquisas sobre placas modeladas pelo MEC, enrijecidas foram feitas devido à importância de se conhecer o seu comportamento em presença de carregamento transversal. Os enrijecedores são, em geral, modelados pelo MEF e, 
portanto, algumas técnicas de combinação entre os métodos numéricos são apresentadas de diversas formas. Como exemplo de trabalhos desenvolvidos neste departamento, citam-se PAIVA (1987), que mostrou associações diversas com estruturas de barras, pilares e vigas e mais recentemente OLIVEIRA NETO (1998), para análise de pavimentos de edifícios em que uma terceira variável de deslocamento é incluída. O equilíbrio é feito apenas entre forças verticais. Estes aspectos facilitam o acoplamento além e aproximar melhor os resultados. Nestes casos, a influência dos enrijecedores foi considerada como uma reação dos mesmos sobre a placa. SILVA (1996) desenvolveu um sistema semelhante ao de PAIVA(1987), porém tratando as placas com as hipóteses de Reissner. PAIVA \& VENTURINI (1985) e PAIVA (1987) formulam a influência da suportagem de lajes cogumelo como forças sobre elas atuantes, transformadas em equações integrais. Posteriormente, PAIVA \& VENTURINI (1987) fizeram a combinação entre placas e grelhas, sendo o equilíbrio na região da interface estabelecido entre forças verticais.

TANAKA \& BERCIN (1997) desenvolveram uma formulação para a análise de placas com enrijecedores de seções transversais variadas, levando-se em conta todas as rigidezes e a excentricidade dos eixos deles em relação aos eixos neutros das placas. Desta forma, ele combina os efeitos de flexão e do estado plano de tensão à placa. Segundo eles, a influência dos enrijecedores irá refletir na formulação de acordo com cada pesquisador. Em seu caso, as rigidezes das barras são incorporadas à equação de deslocamentos das placas finas (fig. 1.1).

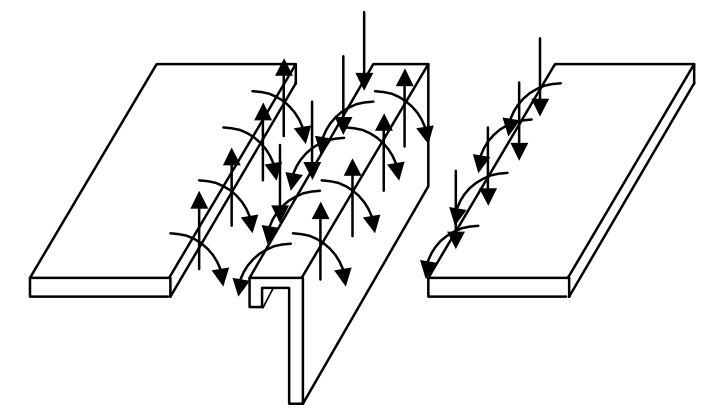

Fig. 1.1 - Exemplo utilizado por TANAKA \& BERCIN (1997)

Outro trabalho que se pode citar a respeito da combinação do efeito de flexão com o do EPT é o de PALERMO(1989) que estudou peças (barras) de seções 
abertas delgadas, analisadas como placas acopladas no espaço. Os efeitos de placa e de chapa são associado na mesma barra toda vez que ela for composta por placas não-coplanares.

Outros pesquisadores também apresentam trabalhos sobre o acoplamento entre regiões modeladas pelo MEC e MEF que não para o caso de pavimentos de edifícios, o que foi de grande valia para este trabalho. Através deles, vários aspectos sobre o problema da combinação entre os métodos a serem cuidados foram previstos para a elaboração do algoritmo desde o seu início. Neste sentido pode citar o trabalho de BREBBIA \& GEORGIOU (1979) examinam a combinação MEC/MEF aplicada a dois problemas elastostáticos de duas formas diferentes. O primeiro método trata a região modelada por elementos de contorno como uma região de elementos finitos. $\mathrm{O}$ segundo trata o elemento finito como elemento de contorno. Concluem que o primeiro método parece ser mais interessante pois pode ser facilmente incorporada a algoritmos de MEC já existentes.

Há trabalhos em que regiões de meio contínuo, 2 ou 3-D, tratadas pelo MEC e barras pelo MEF. Foi o caso do trabalho de CODA et al(1997), em que a análise não-linear também é feita.

MESSAFER \& COATES (1989) analisam placas de várias rigidezes à flexão apoiadas sobre solo elástico semi-infinito, utilizando a combinação MEC/MEF considerando elementos não-conformes. CODA (1993) avaliou este tipo de interação no conjunto solo-estrutura de barras. FERRO \& VENTURINI (1992) formularam a formulação com observação em detalhes particulares e característicos da modelagem de fundações sobre estacas. CALDERÓN \& VENTURINI (1997) apresentam modificação na representação integral de deslocamentos em placas finas, incluindo a influência do substrato sobre elas como integral de domínio proveniente da sua própria representação integral através de três métodos: pela subdivisão do domínio em células, pelo uso do método da reciprocidade dual e por outro processo alternativo também baseado em funções globais. 
BEER (1986) discute a eficiente implementação do elemento de contorno num algoritmo elaborado em elementos finitos já existente para aplicação em interação solo-estrutura. O resultado final desta implementação é avaliado através de exemplos de estruturas modeladas pelos dois métodos simultaneamente. Estes resultados são confrontados com os obtidos para estas mesmas estruturas modeladas pelo MEC.

CHAUDOUET-MIRANDA \& CRISTESCU (1993) demonstram o uso a eficiência da combinação entre o MEC e o MEF na industrial.

\section{1 - OBJETIVO DO PRESENTE TRABALHO}

O objetivo desta pesquisa é desenvolver uma formulação e o respectivo algoritmo numérico, com a devida implementação em microcomputador, para análise de pavimentos dede edifícios. Uma característica principal do desenvolvimento a ser feito é que o tratamento às placas é dado empregando-se o MEC para placas delgadas e os elementos lineares tratados pelo MEF. Em uma segunda fase a estrutura completa do edifício será tratada combinando o elemento desenvolvido com os elementos verticais.

A idéia é contribuir com a gama de trabalhos desenvolvidos no assunto, acrescentando-se, porém, a consideração do efeito da excentricidade do eixo das barras enrijecedoras em relação ao verdadeiro nível do acoplamento com a placa. Escolheu-se utilizar a técnica das sub-regiões ou dos multidomínios, para viabilizar a compatibilização ou acoplamentos dos dois métodos. Diversos aspectos desta técnica são abordados no intuito de facilitar a elaboração de sub-rotina e/ou adaptação de programas, visando utilizá-la para esta ou outra combinação entre métodos de análise estrutural desejada. A partir daí, pode-se proceder a eliminação de graus de liberdade da estrutura durante a análise por condensação estática. 
A programação dos algoritmos propostos foi feita em linguagem FORTRAN para microcomputadores.

\section{2 - APRESENTAÇÃO DO TRABALHO POR CAPÍTULOS}

O trabalho incluirá capítulo (capítulo 2) com conceitos básicos relativos aos problemas de flexão placas delgadas e à aplicação do MEC na resolução deste problema, de forma simplificada, por se tratar de assunto bastante conhecido e estudado (ver, p.e., VENTURINI(1988), PAIVA(1987), OLIVEIRA NETO(1998), CHUEIRI(1994), ALIABADI(1998), BREBBIA \& DOMINGUES(1989), BREBBIA, TELLES \& WRÖBEL(1984), HARTMANN (1991)). Num capítulo seguinte, expõem-se os mesmos aspectos do MEC aplicado ao Estado Plano de Tensões (EPT) (capítulo 3).

No capítulo 4 apresentam-se aspectos sobre o elemento finito de barra utilizado para discretizar os elementos estruturais lineares. Somente os aspectos principais que causarão influência no processo de combinação destes com os elementos estruturais de superfícies modelados pelo MEC serão abordados. A vasta bibliografia sobre o assunto permite que se faça apenas um breve detalhamento destes aspectos (p.e., BATHE(1982), ZIENKEWICZ(1971), CODA(1993)).

Como foi dito, diversos conceitos e aspectos da utilização da técnica de subregiões são discutidos capítulo 5, auxiliando na elaboração de uma sub-rotina a ser incorporada no programa de placas finas, com ênfase às modificações necessárias para adaptá-la aos algoritmos propostos nos capítulos anteriores. Diversos trabalhos e livros dão a orientação para a formulação básica desta técnica para uso geral em engenharia (p.e., VENTURINI (1983), BREBBIA \& DOMINGUES (1989), ALIABADI \& ROOKE (1992), dentre muitos outros), alguns com ênfase para o caso de placas finas (WEARING \& BETTAHAR (1994)). Os aspectos necessários para desenvolvimento de rotina para este fim, são detalhados neste capítulo. São 
apresentados alguns exemplos para demonstração de eficiência e de como tal técnica deve ser usada adequadamente.

O capítulo 6 aborda o acoplamento entre duas ou mais sub-regiões, modeladas pelo MEC e/ou o MEF, através da técnica das sub-regiões. O acoplamento de barras dispostas no domínio ou no contorno da placa é feito, no primeiro caso, entre os nós destas e os nós internos da placa de mesma posição. A solução obtém-se considerando a influência da barras como linhas ou pontos de carga sobre a placa ou subdividindo-se o domínio em estudo de forma adequada, de forma que exista a interação entre as sub-regiões. Exemplos de elementos e estruturas acoplados são apresentados. O efeito de membrana causado pela excentricidade do eixo neutro das barras em relação à superfície neutra da placa é considerado em seguida, com o modelo idealizado através do PTV..

Antecipando-se ao capítulo de conclusões, finalmente apresenta-se o capítulo que abordada os aspectos do processo de condensação estática adaptado ao sistema de equações resultante do acoplamento das sub-regiões de estruturas. Utiliza-se a técnica também utilizada por WILSON(1974) e que se apresenta como uma extensão do processo básico da eliminação de Gauss. 


\section{CAPÍTULO 2 - TEORIA DE KIRCHHOFF E O MEC APLICADO A PLACAS FINAS}

\section{1 - INTRODUÇÃ̃O}

Neste capítulo, objetiva-se analisar os efeitos do carregamento em placas finas -pela teoria de KIRCHHOFF - a partir de uma formulação do Método dos Elementos de Contorno (MEC). O algoritmo desenvolvido por CHUEIRI(1994) baseado nesta formulação é utilizado como uma rotina básica para o cálculo de estruturas formadas por placas delgadas.

\section{2 - ANÁliSE DE PLACAS FINAS PELA TEORIA DE KIRCHHOFF}

A análise de placas delgadas com base na teoria de Kirchhoff, a chamada Teoria Clássica, para pequenos deslocamentos, é uma simplificação do problema tridimensional na Teoria da Elasticidade. Para placas delgadas sob carregamento transversal, listam-se aqui as hipóteses básicas de cálculo estabelecidas nesta teoria: 
-o material de que é composta a placa é suposto homogêneo, isotrópico e elástico linear;

-a espessura da placa t é pequena se comparada às suas outras dimensões;

-os deslocamentos verticais resultantes desse carregamento são pequenos em comparação à espessura $\underline{\mathbf{t}}$ da placa;

-os deslocamentos horizontais dos pontos do plano médio da placa são negligenciados pois assume-se este ser a superfície neutra;

-as seções transversais inicialmente planas e normais à superfície neutra assim permanecem após a deformação da placa;

-as tensões normais $\sigma_{33}$, perpendiculares ao plano da placa, podem ser desprezadas (vide fig. 2.1) em presença das demais componentes de tensão.

A fig. 2.1 mostra os sentidos adotados como positivos dos eixos coordenados e componentes de tensão em um elemento de placa. Para a análise do problema de placas, pode-se obter as seguintes equações, com base nas condições básicas acima listadas e de acordo com o sistema ortogonal $\mathbf{x}_{1} \mathbf{x}_{2} \mathbf{x}_{3}$ com origem na superfície média:

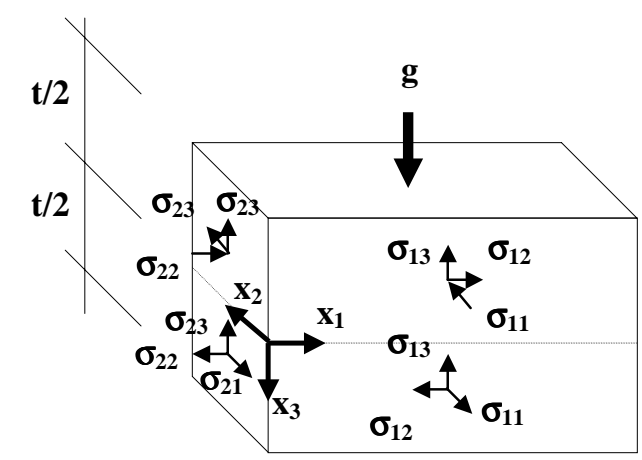

Fig. 2.1 - Tensões, forças e esforços sobre elemento de placa

Seguem-se as equações básicas necessárias para se formular a análise de placas delgadas com base nas hipóteses estabelecidas nesta teoria. 


\section{3 - DESLOCAMENTOS $\left(\mathrm{u}_{\mathrm{i}}\right)$ :}

Sobre as componentes de deslocamento $\mathbf{u}_{\mathrm{i}}$ de um ponto, sendo $\mathbf{i}=\mathbf{1 , 3}$ e levandose em conta as hipóteses básicas adotadas para placas finas, pode-se dizer que:

$$
\mathbf{u}_{3}=\mathbf{w}\left(\mathbf{x}_{1}, \mathbf{x}_{2}\right)
$$

onde $\mathbf{w}\left(\mathbf{x}_{1}, \mathbf{x}_{2}\right)$ representa os deslocamentos nos pontos do plano médio da placa, definido no plano $\mathbf{x}_{1} \mathbf{x}_{2}$.

Observando-se o elemento de placa da fig. 2.2 e analisando-o no plano $\mathbf{x}_{1} \mathbf{x}_{3}$ após a deformação, a superfície média apresenta uma rotação $\mathbf{w},{ }_{1}$ (a vírgula indicando derivação) num ponto $\mathbf{P}$ de uma determinada seção transversal que desloca $\mathbf{u}_{3}$ na direção $\mathbf{x}_{3}$. Após analisar-se o elemento da mesma forma, agora no plano $\mathbf{x}_{2} \mathbf{x}_{3}$, pode-se concluir que:

$$
u_{i}=-x_{3},,_{i} \quad i=1,2
$$
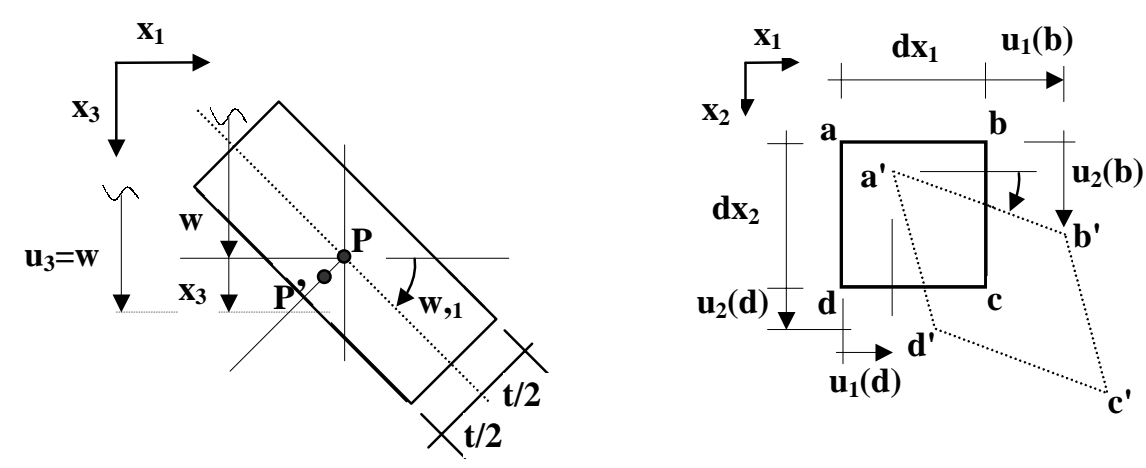

Fig. 2.2 - Componentes de deslocamento de elemento de placa 


\section{4 - DEFORMAÇÕES $\left(\varepsilon_{\mathrm{ij}}\right)$}

Analisando-se o elemento de placa abcd mostrado na fig.(2.2) no nível da superfície média após a mudança de forma, pode-se escrever as componentes de deslocamentos, por exemplo, dos pontos b e d:

$$
\begin{aligned}
& u_{1}(b)=u_{1}(a)+u_{1}, 1 d x_{1} \\
& u_{2}(b)=u_{2}(a)+u_{2}, 1 d x_{1}
\end{aligned}
$$

$\mathrm{e}$

$$
\begin{aligned}
& u_{1}(d)=u_{1}(a)+u_{1}, 2 d x_{2} \\
& u_{2}(d)=u_{2}(a)+u_{2}, 2 d x_{2}
\end{aligned}
$$

Daí,

$$
\begin{aligned}
& \varepsilon_{11}=\frac{u_{1}(b)-u_{1}(a)}{d x_{1}}=u_{1}, 1 \\
& \varepsilon_{22}=\frac{u_{2}(b)-u_{2}(a)}{d x_{2}}=u_{2}, 2
\end{aligned}
$$

e

$$
\varepsilon_{12}=\varepsilon_{21}=\frac{u_{1}, 2+u_{2}, 1}{2}
$$

De uma forma geral, obtém-se:

$$
\varepsilon_{i j}=\frac{u_{i},{ }_{j}+u_{j}, i}{2}
$$

ou, em termos de deslocamentos e a partir da eq. 2.2

$$
\boldsymbol{\varepsilon}_{\mathrm{ij}}=-\mathbf{x}_{\mathbf{3}} \mathbf{w}, \quad \mathrm{i}, \mathrm{j}=1,2
$$




\section{5 - TENSÕES $\left(\sigma_{\mathrm{ij}}\right)$}

De acordo com o a lei de Hooke as componentes de tensão são obtidas através da equação (na sua forma indicial):

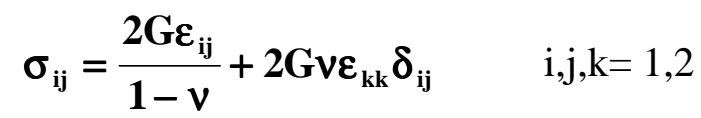

onde

$$
\mathbf{G}=\frac{\mathbf{E}}{2(1+v)}
$$

é o módulo de elasticidade transversal, bem como $\mathbf{E}$ é o módulo de elasticidade do material da placa, $\boldsymbol{v}$ é o coeficiente de Poisson e $\boldsymbol{\delta}_{\mathrm{ij}}$ é o delta de Kronecker

Em termos de deslocamentos, as componentes de tensão podem ser expressas por:

$$
\sigma_{i j}=\frac{-E x_{3}}{\left(1-v^{2}\right)}\left[v w,_{k k} \delta_{i j}+(1-v) w_{i j}\right] \quad i, j, k=1,2
$$

\section{6 - COMPONENTES ESFORÇOS $\left(\mathbf{m}_{\mathrm{ij}}\right.$ e $\left.\mathrm{q}_{\mathrm{i}}\right)$}

As componentes dos momentos fletor e volvente e também da força cortante são obtidas formulando-se o equilíbrio de um elemento de placa. Com base nas hipóteses básicas, estabelece-se que a distribuição de tensões pela espessura da placa é linear, podendo-se assim calcular suas resultantes e as respectivas componentes de momento e força cortante por unidade de comprimento, na forma:

$$
\mathbf{m}_{\mathrm{ij}}=\int_{-t / 2}^{t / 2} \sigma_{\mathrm{ij}} \mathbf{x}_{3} d \mathbf{x}_{3}
$$




$$
q_{i}=\int_{-t / 2}^{t / 2} \sigma_{i 3} d x_{3}
$$

Integrando-se as equações acima e fazendo as devidas transformações, chega-se às relações:

$$
\begin{array}{ll}
\mathbf{m}_{\mathrm{ij}}=-\mathbf{D}\left[\mathbf{v w},_{\mathbf{k k}} \boldsymbol{\delta}_{\mathrm{ij}}+(\mathbf{1}-\mathbf{v}) \mathbf{w},_{\mathrm{ij}}\right] & \\
\mathbf{q}_{\mathrm{i}}=-\mathbf{D w},_{\mathrm{kki}} & \mathrm{i}, \mathrm{j}, \mathrm{k}=1,2
\end{array}
$$

onde $\quad \mathbf{D}=\frac{\mathbf{E t}^{3}}{12\left(1-v^{2}\right)}$

representa a rigidez da placa à flexão.

\section{7 - EQUAÇÃO DIFERENCIAL DE PLACAS}

A análise da maioria das configurações de placa consiste em resolver uma equação diferencial dada em termos de deslocamentos, cargas aplicadas e rigidez da placa.

Como

$$
\mathbf{m}_{\mathrm{ij}},_{\mathrm{i}}=-\mathbf{D w},_{\mathrm{kkj}}=\mathbf{q}_{\mathbf{j}}
$$

$\mathrm{e}$

$$
\mathbf{q}_{\mathrm{i}}, \boldsymbol{i}_{\mathrm{i}}=-\mathbf{g}
$$

chega-se a 


$$
\mathbf{m}_{\mathrm{ij}},_{\mathrm{ij}}=-\mathbf{g}
$$

$\mathrm{e}$

$$
-\mathbf{D w},_{\mathbf{k k l l}}=-\mathbf{g} \Rightarrow \mathbf{w},_{\mathbf{k k l l}}=\frac{\mathbf{g}}{\mathbf{D}}
$$

$\mathrm{ou}$

$$
\nabla^{2} \nabla^{2} \mathbf{w}=\frac{\mathbf{g}}{\mathbf{D}} \quad \text { ou } \quad \nabla^{4} \mathbf{w}=\frac{\mathbf{g}}{\mathbf{D}}
$$

para $\mathbf{i}, \mathbf{j}, \mathbf{k}, \mathbf{l}=\mathbf{1 , 2}$ e $\nabla^{2}=\frac{\mathbf{d}^{2}()}{\mathbf{d x}_{1}^{2}}+\frac{\mathbf{d}^{2}()}{\mathbf{d x}_{2}^{2}} \quad$ (operador de Laplace).

A eq.(2.14) é a equação diferencial de placas, que relaciona o carregamento e os deslocamentos.

\section{8 - VALORES DE CONTORNO}

Pode-se obter os esforços em placas com relação a um sistema genérico de coordenadas $\overrightarrow{\mathbf{n}}$ e $\overrightarrow{\mathbf{s}}$. As componentes de cisalhamento e momento nas direções normal ( $\mathbf{m}_{\mathrm{n}}$, o momento fletor) e tangente $\overrightarrow{\mathbf{s}}\left(\mathbf{m}_{\mathrm{ns}}\right.$, o momento volvente) associadas à superfície analisada são também calculadas pelo estabelecimento do equilíbrio de um elemento de placa, cujas faces laterais são coincididas com as com os planos $\mathbf{x}_{1} \mathbf{x}_{3}$, $\mathbf{x}_{2} \mathbf{x}_{3}$, e $\mathbf{s} \mathbf{x}_{3}$ (fig.(2.3)). Obtém-se, portanto, considerando-se que as componentes $\mathbf{m}_{\mathbf{i j}} \mathrm{e}$ $\mathbf{q}_{\mathbf{i}}$ são uniformemente distribuídas ao longo dessas faces:

$$
\begin{aligned}
& \mathbf{m}_{\mathbf{n}}=\mathbf{m}_{\mathrm{ij}} \mathbf{n}_{\mathbf{i}} \mathbf{n}_{\mathbf{j}} \\
& \mathbf{m}_{\mathrm{ns}}=\mathbf{m}_{\mathrm{ij}} \mathbf{n}_{\mathrm{i}} \mathbf{s}_{\mathbf{j}} \\
& \mathbf{q}_{\mathbf{n}}=\mathbf{q}_{\mathrm{i}} \mathbf{n}_{\mathbf{i}} \quad \mathrm{i}, \mathrm{j}=1,2
\end{aligned}
$$


sendo $\mathbf{n}_{\mathrm{i}}$ e $\mathbf{s}_{\mathrm{i}}$ os cossenos diretores dos versores $\overrightarrow{\mathbf{n}}$ e $\overrightarrow{\mathbf{s}}$.

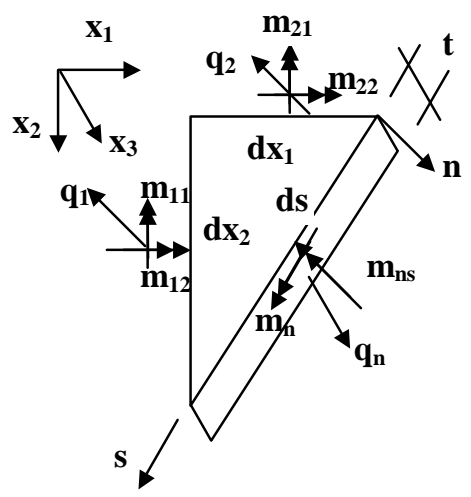

Fig. 2.3 - Componentes de esforços num elemento de placa

\section{8 - CORTANTE EQUIVALENTE $\left(\mathrm{V}_{\mathrm{n}}\right)$}

No contorno da placa existem três valores de forças de superfície (eqs. 2.15 a 2.17) para os quais há três valores de deslocamentos a eles relacionados $(\mathbf{w}, \mathbf{w}, \mathrm{n}$ e $\mathbf{w}$,s). Sendo a equação de equilíbrio do quarto grau o número de valores possíveis é quarto (ver FRANGI \& GIUGGIANI (1999a e b). Assim, torna-se necessário eliminar uma força de superfície, bem como o deslocamento a ela relacionado, por ser diretamente dependente das demais.

$$
\mathbf{W}, \mathrm{s}
$$

Os valores de contorno relativas a $\mathbf{q}_{\mathrm{n}}$ e $\mathbf{m}_{\mathrm{ns}}$ podem ser agrupadas recebendo a denominação de cortante equivalente $\mathbf{V}_{\mathrm{n}}$. Isto pode ser obtido através da análise do elemento infinitesimal ilustrado na fig.(2.4). Este elemento tem uma resultante de momento $\mathbf{M}_{\mathrm{ns}}$ que vale $\mathbf{M}_{\mathrm{ns}}=\mathbf{m}_{\mathrm{ns}} \mathbf{d s}$ e que pode ser representada por um binário de forças, cada uma valendo $\mathbf{m}_{\mathrm{ns}}$, aplicadas nas extremidades do elemento. Analisandose agora dois elementos consecutivos, num ponto do lado comum a ambos, resultará uma força $\mathbf{m}_{\mathrm{n}}$, s $\mathbf{d s}$ que se somará à força cortante resultante $\mathbf{q}_{\mathrm{n}} \mathbf{d} \mathbf{s}$ no ponto observado. Daí, surge a força cortante equivalente $\mathbf{V}_{\mathbf{n}} \mathbf{d s}=\mathbf{q}_{\mathbf{n}} \mathbf{d s}+\mathbf{m}_{\mathbf{n s} s \mathbf{s}} \mathbf{d s}$, que por unidade de comprimento vale: 
$\mathbf{V}_{\mathrm{n}}=\mathbf{q}_{\mathrm{n}}+\mathbf{m}_{\mathrm{ns}}, \mathrm{s}$

Os valores de contorno restantes são, portanto, $\mathbf{m}_{\mathrm{n}}, \mathbf{V}_{\mathrm{n}}, \mathbf{w}$ e $\mathbf{w}, \mathrm{n}$.
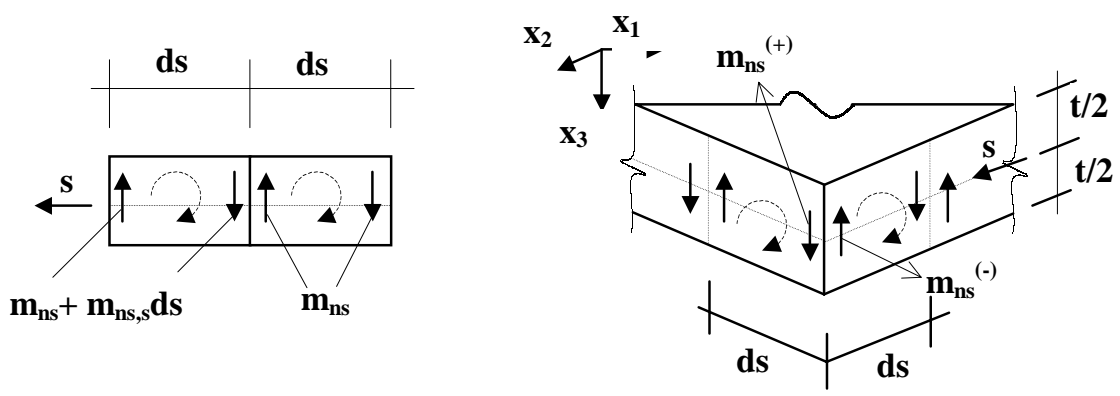

Fig. 2.4 - Momento volvente $\left(\mathrm{m}_{\mathrm{ns}}\right)$ no contorno

\section{9 - FORÇAS CONCENTRADAS NOS CANTOS $\left(\mathbf{R}_{\mathrm{c}}\right)$}

Seguindo-se a mesma análise feita no item $\mathbf{2 . 8}$, onde se substitui o momento volvente por binário de forças para se obter a cortante equivalente $\mathbf{V}_{\mathbf{n}}$, pode-se verificar também o surgimento de forças concentradas nos cantos, provenientes dos lados que os formam. Elas valem $\mathbf{m}_{\mathrm{ns}}{ }^{(+)}$ou $\mathbf{m}_{\mathrm{ns}}{ }^{(-)}\left(\mathbf{m}_{12}\right.$ ou $\mathbf{m}_{21}$ se as bordas são paralelas aos eixos $\mathbf{x}_{1}$ ou $\mathbf{x}_{2}$ ). Os sinais de (+) e (-) indicam o valor de $\mathbf{m}_{\mathrm{ns}}$ posterior e anterior ao canto $\underline{\mathbf{i}}$, respectivamente (fig.(2.4)). Portanto, forças externas $\mathbf{R}_{\mathrm{c}}$ devem ser aplicadas em cada canto $\underline{\mathbf{i}}$ quando para imobilizá-los e valem:

$$
\mathbf{R}_{\mathbf{c}} \mathbf{i}=\mathbf{m}_{\mathrm{ns}}^{(+)}-\mathbf{m}_{\mathrm{ns}}^{(-)}
$$

\subsection{0 - SOLUÇÃO FUNDAMENTAL (*)}

Para a formulação do MEC para o problema de flexão de placas, é necessário obter-se a solução fundamental que se define como o deslocamento w num 
determinado ponto $\mathbf{p}$ causado por uma força unitária aplicada à placa de domínio infinito, num ponto de carregamento q. Esta solução é classicamente dada pela solução do problema de uma placa circular carregada no seu centro por uma carga unitária que matematicamente pode ser representada pela distribuição do delta de $\operatorname{Dirac}(\Delta(\mathrm{q}, \mathrm{p}))$, cujas propriedades são as seguintes:

$$
\begin{array}{lll}
\Delta(\mathrm{q}, \mathrm{p})=0 & \text { para } & \mathrm{p} \neq \mathrm{q} \\
\Delta(\mathrm{q}, \mathrm{p})=\infty & \text { para } & \mathrm{p}=\mathrm{q}
\end{array}
$$

$\mathrm{e}$

$$
\int_{\Omega} \Delta(\mathrm{q}, \mathrm{p}) \mathbf{d} \Omega=\mathbf{1}
$$

Daí, definindo-se $\phi(p)$ como uma função contínua, tem-se

$$
\int_{\Omega} \phi(p) \Delta(q, p) d \Omega=\phi(q)
$$

que significa que a resultante da carga distribuída $\Delta(\mathrm{q}, \mathrm{p})$, aplicada em uma área infinitamente pequena, representa uma carga unitária aplicada no ponto q.

A solução do problema descrito acima resulta em:

$$
w^{*}(\mathbf{q}, \mathbf{p})=\frac{1}{8 \pi D} \mathbf{r}^{2}\left(\ln \mathbf{r}-\frac{1}{2}\right)
$$

sendo $\underline{\mathbf{r}}$ a distância entre os pontos $\mathbf{p}$ (ponto de resposta) e $\mathbf{q}$ (ponto de carga) definidos sobre a placa circular de raio infinito, valendo:

$$
\mathbf{r}=\left\{\left(\mathbf{x}_{\mathbf{i}}(\mathbf{p})-\mathbf{x}_{\mathbf{i}}(\mathbf{q})\right)^{2}\right\}^{1 / 2}
$$

Ainda da eq.(2.20) pode-se obter: 


$$
\begin{aligned}
& w_{,_{n}} *(q, p)=\frac{d\left(w^{*}(q, p)\right)}{d r} \frac{d r}{d n}=\frac{1}{4 \pi D} r \ln r\left(r, n_{i} n_{i}\right) \\
& m_{n} *(q, p)=\frac{-1}{4 \pi}\left[(1+v) \ln r+(1-v)\left(r, n_{i}\right)^{2}+v\right] \\
& m_{n s} *(q, p)=\frac{-1}{4 \pi}(1-v)\left(r, n_{i}\right)\left(r, s_{j} s_{j}\right) \\
& V_{n} *(q, p)=\frac{\left(r, n_{i}\right)}{4 \pi}\left[2(1-v)\left(r, s_{j} s_{j}\right)^{2}-3+v\right] \\
& R_{c i} *(q, p)=m_{n s}^{*(+)}-m_{n s}^{*(-)}
\end{aligned}
$$

No próximo item, mostra-se que será necessário conhecerem-se as derivadas dos deslocamentos e esforços fundamentais acima calculados, em relação a uma direção $\overrightarrow{\mathbf{m}}$ de origem em q. Neste caso, são necessárias as seguintes relações:

$$
\mathbf{r}=\left\{\left(\mathbf{x}_{\mathrm{i}}(\mathbf{q})-\mathbf{x}_{\mathbf{i}}(\mathbf{p})\right)^{2}\right\}^{1 / 2}
$$

e

$$
\begin{gathered}
\frac{\partial \mathbf{r}}{\partial \mathbf{x}_{i}(\mathbf{q})}=\frac{\mathbf{x}_{\mathrm{i}}(\mathbf{q})-\mathbf{x}_{\mathrm{i}}(\mathbf{p})}{\mathbf{r}}=-\mathbf{r},_{\mathrm{i}} \\
\frac{\partial \mathbf{r}_{\mathrm{i}}}{\partial \mathbf{x}_{\mathrm{j}}(\mathbf{q})}=-\frac{\delta_{\mathrm{ij}}-\mathbf{r},_{\mathrm{i}} \mathbf{r},_{j}}{\mathbf{r}}=-\mathbf{r},_{\mathrm{ij}}
\end{gathered}
$$

portanto, pode-se obter

$$
\frac{\partial w^{*}}{\partial \mathbf{m}}=\frac{d w^{*}}{d \mathbf{r}} \cdot \frac{\partial \mathbf{r}}{\partial \mathbf{m}}=\frac{d w^{*}}{d \mathbf{r}} \cdot\left[\frac{\partial \mathbf{r}}{\partial x_{i}(q)} \cdot \frac{\partial x_{i}(q)}{\partial \mathbf{m}}\right]=\frac{d w^{*}}{d \mathbf{r}} \cdot\left[-r_{i} \cdot m_{i}\right]
$$




$$
\begin{aligned}
& \mathbf{w},{ }_{\mathrm{m}} *(\mathbf{q}, \mathbf{p})=-\frac{1}{4 \pi \mathrm{D}} \mathbf{r} \ln r\left(\mathbf{r}, \mathrm{m}_{\mathrm{i}}\right) \\
& \mathbf{w},{ }_{n m} *(q, p)=-\frac{1}{4 \pi D}\left[\left(r, m_{i}\right)\left(r,{ }_{j} n_{j}\right)+\left(m_{i} n_{i}\right) \ln r\right] \\
& \mathbf{w},{ }_{\mathrm{sm}} *(\mathbf{q}, \mathbf{p})=-\frac{1}{4 \pi \mathrm{D}}\left[\left(\mathbf{r}, \mathbf{m}_{\mathrm{i}}\right)\left(\mathbf{r},{ }_{\mathrm{j}} \mathbf{s}_{\mathbf{j}}\right)+\left(\mathbf{m}_{\mathrm{i}} \mathbf{s}_{\mathrm{i}}\right) \ln \mathbf{r}\right]
\end{aligned}
$$

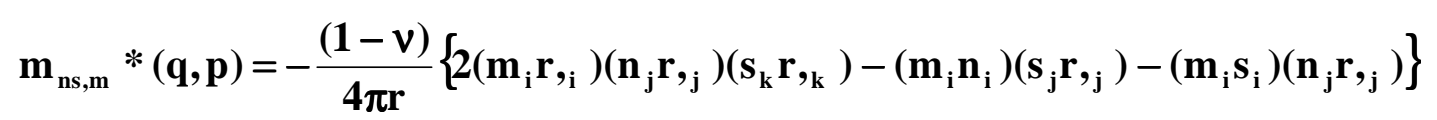

\subsection{1 - REPRESENTAÇÃO INTEGRAL PARA PONTOS DO DOMÍNIO}

É necessário obter-se a formulação integral que define o problema de placas finas o que é feito a partir do método dos resíduos ponderados ou pelo Teorema da Reciprocidade de $\boldsymbol{B e t t i .}$

Toma-se a placa da fig.(2.5) de domínio $\Omega$ como parte de um domínio infinito $\left(\boldsymbol{\Omega}_{\infty}\right)$. Considere-se ainda um carregamento distribuído $\mathbf{g}$ aplicado sobre a região $\boldsymbol{\Omega}_{\mathrm{g}}$. $\mathrm{O}$ carregamento $\mathbf{g}^{*}$ (da solução fundamental correspondente) aplicado à placa infinita produz deslocamentos transversais fundamentais já deduzidos $\mathbf{w}^{*}$ e os correspondentes estados de tensão $\sigma_{\mathrm{ij}} *$ e deformação $\varepsilon_{\mathrm{ij}} *$. Analogamente para 0 problema real, os seguintes valores estão relacionados: $\mathbf{g}, \mathbf{w}, \boldsymbol{\sigma}_{\mathrm{ij}}$ e $\boldsymbol{\varepsilon}_{\mathrm{ij}}$. Para esses dois estados de solicitação válidos no domínio $\Omega$, a seguinte relação de reciprocidade pode ser escrita:

$$
\int_{V} \sigma_{\mathrm{ij}} * \varepsilon_{\mathrm{ij}} \mathrm{dV}=\int_{\mathrm{V}} \sigma_{\mathrm{ij}} \varepsilon_{\mathrm{ij}} * d V
$$




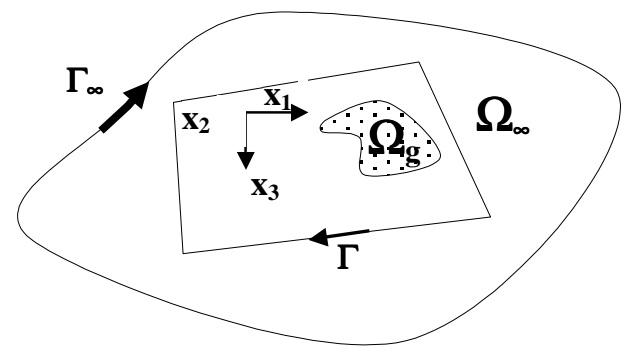

Fig. 2.5 - Domínio de placas

onde $\mathbf{V}$ é o volume do elemento considerado como um corpo tridimensional.

A carga distribuída $\mathbf{g}^{*}$ representa o carregamento fundamental arbitrada como unitária e cuja representação matemática é a distribuição delta de Dirac $\Delta(\mathrm{q}, \mathrm{p})$. Portanto, tensões, deformações, deslocamentos, momentos e forças cortantes associados a este carregamento serão indicados com o símbolo (*).

Com base nas hipóteses da teoria de Kichhoff e juntamente com a integração da eq.(2.33) ao longo da espessura da placa, tem-se:

$$
\int_{\Omega} \mathbf{m}_{\mathrm{ij}} * \mathbf{w},{ }_{\mathrm{ij}} \mathrm{d} \Omega=\int_{\Omega} \mathbf{m}_{\mathrm{ij}} \mathbf{w},{ }_{\mathrm{ij}} * \mathrm{~d} \Omega
$$

cuja dupla integração por partes fornece:

$$
\begin{aligned}
& \int_{\Gamma}\left(m_{\mathrm{ij}} \mathbf{w}_{\mathbf{i}_{\mathrm{i}}}{ }^{*} \mathbf{n}_{\mathrm{j}}-\mathbf{m}_{\mathrm{ij}},{ }_{\mathrm{j}} \mathbf{w},{ }_{\mathrm{j}}{ }^{*} \mathbf{n}_{\mathrm{i}}\right) \mathrm{d} \Gamma+\int_{\Omega} \mathbf{m}_{\mathrm{ij}},_{\mathrm{ij}} \mathbf{w} * \mathbf{d} \boldsymbol{\Omega}= \\
& \int_{\Gamma}\left(\mathbf{m}_{\mathrm{ij}} * \mathbf{w}, \mathbf{n}_{\mathrm{i}}-\mathbf{m}_{\mathrm{ij}},{ }_{\mathrm{j}}^{*} \mathbf{w}, \mathbf{n}_{\mathrm{j}}\right) \mathrm{d} \Gamma+\int_{\Omega} \mathbf{m}_{\mathrm{ij}},_{\mathrm{ij}} * \mathbf{w d} \Omega
\end{aligned}
$$

Considerando-se as equações de equilíbrio que envolvem o valor de $\mathbf{m}_{\mathrm{ij}}$ (eqs. 2.10 e 2.12)e também que:

$$
\mathbf{w},{ }_{i}=\mathbf{w},{ }_{n} \mathbf{n}_{\mathbf{i}}+\mathbf{w},{ }_{\mathrm{s}} \mathbf{s}_{\mathbf{i}}
$$


e, analiticamente

$\mathbf{w},{ }_{i}^{*}=\mathbf{w}, \mathbf{n}^{*} \mathbf{n}_{\mathbf{i}}{ }^{*}+\mathbf{w}, \mathbf{S}_{\mathbf{i}}{ }^{*}$

a eq.(2.34) é reescrita na forma:

$$
\begin{aligned}
& -\int_{\Gamma}\left(\mathbf{m}_{\mathbf{n}} \mathbf{w}, \mathbf{n}_{\mathbf{n}}{ }^{*}+\mathbf{m}_{\mathrm{ns}} \mathbf{w},{ }_{\mathrm{s}}^{*}-\mathbf{q}_{\mathrm{n}} \mathbf{w} *\right) \mathrm{d} \Gamma+\int_{\Omega} \mathbf{g w} * \mathrm{~d} \Omega= \\
& -\int_{\Gamma}\left(\mathbf{m}_{\mathrm{n}} * \mathbf{w},{ }_{\mathrm{n}}+\mathbf{m}_{\mathrm{ns}} * \mathbf{w},{ }_{\mathrm{s}}-\mathbf{q}_{\mathrm{n}} * \mathrm{w}\right) \mathrm{d} \Gamma+\int_{\Omega} \mathrm{g} * \mathbf{w d} \Omega
\end{aligned}
$$

Analisando-se o primeiro membro da eq.(2.35), fazendo-se a integração por partes do segundo termo da integral sobre o contorno, obtém-se

$$
\int_{\Gamma}\left(\mathbf{m}_{\mathrm{ns}} \mathbf{w},,_{\mathrm{s}}^{*}\right) \mathrm{d} \Gamma=\left[\mathbf{m}_{\mathrm{ns}} \mathbf{w} *\right]_{\Gamma_{1}}^{\Gamma_{2}}-\int_{\Gamma}\left(\mathbf{m}_{\mathrm{ns}}, \mathrm{s}, \mathrm{W}\right) \mathrm{d} \Gamma
$$

O termo $\mathbf{m}_{\mathbf{n s}} \mathbf{w}^{*}$ da equação acima, resultante da integração sobre todo o contorno, difere de zero somente onde há descontinuidade da normal, i.e., nos cantos, e vale:

$$
\mathbf{m}_{\mathrm{ns}} \mathbf{W}^{*}=-\sum_{\mathrm{i}=1}^{\mathrm{Nc}} \mathbf{R}_{\mathrm{ci}} \mathbf{w}_{\mathrm{ci}} *
$$

onde $\mathbf{N}_{\mathrm{c}}$ é o número de cantos da placa, $\mathbf{R}_{\mathrm{ci}}$ e $\mathbf{w}_{\mathrm{ci}}{ }^{*}$ são a reação e o valor de $\mathbf{w}^{*}$ no canto $\mathrm{i}$ respectivamente. $\mathrm{O}$ mesmo procedimento e conceitos podem ser aplicados ao segundo membro da eq.(2.35).

Deve-se lembrar que os deslocamentos, força cortante e momentos relacionados com a solução fundamental dependem da posição de ambos os pontos de colocação e o ponto fonte $(\mathbf{q}, \mathbf{p})$, de acordo com a definição de solução 
fundamental. Por outro lado, deslocamentos, forças cortantes e momentos relacionados com o problema real, dependem somente do ponto de colocação (p).

Substituindo-se eq.(2.36) e considerando-se a eq.(2.37) e o valor de $\mathbf{V}_{\mathrm{n}}$ (eq.(2.18)) na eq.(2.35), e ainda, lembrando-se que $\mathbf{g}^{*}$ representa o carregamento do problema fundamental dado pelo delta Dirac (ver item 2.10), isto é:

$$
\int_{\Omega} \Delta(\mathrm{q}, \mathrm{p}) \mathbf{w}(\mathrm{p}) \mathbf{d} \Omega=\mathbf{w}(\mathrm{q})
$$

obtém-se a representação integral dos deslocamentos dos pontos do domínio de placas sob carregamento $\mathbf{g}$ aplicado sobre uma região $\boldsymbol{\Omega}_{\mathrm{g}}$ que é:

$$
\begin{aligned}
& \mathbf{w}(\mathbf{q})+\int_{\Gamma}\left[\mathbf{V}_{\mathbf{n}} *(\mathbf{q}, \mathbf{P}) \mathbf{w}(\mathbf{P})-\mathbf{m}_{\mathrm{n}} *(\mathbf{q}, \mathbf{P}) \mathbf{w}, \mathbf{n}(\mathbf{P})\right] \mathrm{d} \Gamma(\mathbf{P})+\sum_{\mathrm{i}=1}^{\mathbf{N}_{\mathrm{c}}} \mathbf{R}_{\mathrm{ci}} *(\mathbf{q}, \mathbf{P}) \mathbf{w}_{\mathrm{ci}}(\mathbf{P})= \\
& \int_{\Gamma}\left(\mathbf{V}_{\mathrm{n}}(\mathbf{P}) \mathbf{w} *(\mathbf{q}, \mathbf{P})-\mathbf{m}_{\mathrm{n}}(\mathbf{P}) \mathbf{w},,_{\mathrm{n}} *(\mathbf{q}, \mathbf{P})\right) \mathrm{d} \Gamma(\mathbf{P})+ \\
& \sum_{\mathrm{i}=1}^{\mathbf{N}_{\mathrm{c}}} \mathbf{R}_{\mathrm{ci}}(\mathbf{P}) \mathbf{w}_{\mathrm{ci}} *(\mathbf{q}, \mathbf{P})+\int_{\Omega \mathrm{g}} \mathbf{g}(\mathbf{p}) \mathbf{w} *(\mathbf{q}, \mathbf{P}) \mathrm{d} \Omega_{\mathrm{g}}(\mathbf{p})
\end{aligned}
$$

que envolve os valores fundamentais listados nas eq. 2.20 e 2.22 a 2.26 e depende das variáveis de contorno $\mathbf{m}_{\mathrm{n}}(\mathbf{P}), \mathbf{V}_{\mathrm{n}}(\mathbf{P}), \mathbf{w}(\mathbf{P}), \mathbf{w},{ }_{\mathrm{n}}(\mathbf{P}), \mathbf{w}_{\mathrm{ci}}(\mathbf{P})$ e $\mathbf{R}_{\mathrm{ci}}(\mathbf{P})$.

Conforme já dito, é interessante escrever-se a representação integral relativa à derivada do deslocamento de um ponto $\mathbf{q}, \mathbf{w}(\mathbf{q})$, em relação a uma coordenada $\mathbf{m}$ de um sistema de coordenadas cartesianas $(\mathbf{m}, \mathbf{u})$ de origem em $\mathbf{q}$ : 


$$
\begin{aligned}
& \frac{\partial \mathbf{w}}{\partial \mathrm{m}}(\mathbf{q})+\int_{\Gamma}\left[\frac{\partial \mathbf{V}_{\mathbf{n}}^{*}}{\partial \mathrm{m}}(\mathbf{q}, \mathbf{P}) \mathbf{w}(\mathbf{P})-\frac{\partial \mathbf{m}_{\mathbf{n}}^{*}}{\partial \mathbf{m}}(\mathbf{q}, \mathbf{P}) \mathbf{w},_{\mathbf{n}}(\mathbf{P})\right] \mathbf{d} \Gamma(\mathbf{P})+\sum_{\mathrm{i}=1}^{\mathbf{N}_{\mathrm{c}}} \frac{\partial \mathbf{R}_{\mathrm{ci}}^{*}}{\partial \mathrm{m}}(\mathbf{q}, \mathbf{P}) \mathbf{w}_{\mathrm{ci}}(\mathbf{P})= \\
& \int_{\Gamma}\left(\mathbf{V}_{\mathbf{n}}(\mathbf{P}) \frac{\partial \mathbf{w}^{*}}{\partial \mathbf{m}}(\mathbf{q}, \mathbf{P})-\mathbf{m}_{\mathbf{n}}(\mathbf{P}) \frac{\partial \mathbf{w}_{\mathbf{n}_{\mathbf{n}}}^{*}}{\partial \mathbf{m}}(\mathbf{q}, \mathbf{P})\right) \mathrm{d} \Gamma(\mathbf{P})+ \\
& \sum_{i=1}^{N_{c}} R_{c i}(P) \frac{\partial w_{c i}^{*}}{\partial m}(q, P)+\int_{\Omega g} g(p) \frac{\partial w^{*}}{\partial m}(q, p) d \Omega_{g}(p)
\end{aligned}
$$

\subsection{2 - REPRESENTAÇÃO INTEGRAL PARA PONTOS DO CONTORNO}

Através da eq.(2.38) pode-se obter o deslocamento de um certo ponto do domínio. Torna-se necessário, porém, obter-se a formulação para o problema pelo MEC, relacionando deslocamentos e esforços quando o ponto fonte $\mathbf{q}$ situa-se sobre o contorno.Usa-se o seguinte artifício para tal: o domínio da placa é acrescido de um setor de raio $\xi$ centrado em $\mathbf{q}$ (fig.(2.6)). Definem-se, assim, novos domínio $\left(\boldsymbol{\Omega}+\boldsymbol{\Omega}_{\xi}\right)$ e contorno $\left(\Gamma-\bar{\Gamma}+\Gamma_{\xi}\right)$ que modificam a eq.(2.38). O deslocamento $\mathbf{w}(\mathbf{Q})$ é então calculado pela equação abaixo, considerando-se agora que $\mathbf{Q}$ é um ponto no contorno, e portanto, o raio $\xi$ tende a zero:

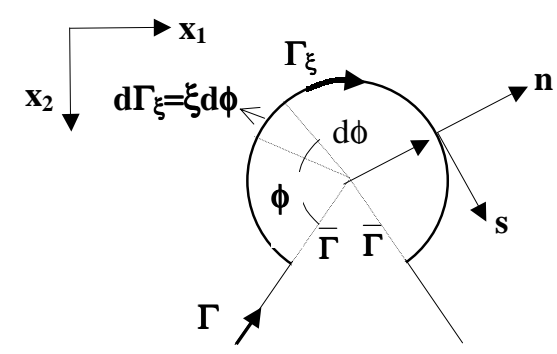

Fig. 2.6 - Ponto do Contorno 


$$
\begin{aligned}
& \mathbf{w}(\mathbf{Q})+\lim _{\bar{\Gamma} \rightarrow 0} \int_{\Gamma-\bar{\Gamma}}\left(\mathbf{V}_{\mathbf{n}} *(\mathbf{Q}, \mathbf{P}) \mathbf{w}(\mathbf{P})-\mathbf{m}_{\mathbf{n}} *(\mathbf{Q}, \mathbf{P}) \mathbf{w},,_{\mathbf{n}}(\mathbf{P})\right) \mathbf{d} \Gamma(\mathbf{P})+ \\
& \lim _{\xi \rightarrow 0} \int_{\Gamma_{\xi}}\left(V_{\mathbf{n}} *(\mathbf{Q}, \mathbf{P}) \mathbf{w}(\mathbf{P})-\mathbf{m}_{\mathbf{n}} *(\mathbf{Q}, \mathbf{P}) \mathbf{w},{ }_{\mathbf{n}}(\mathbf{P})\right) \mathbf{d} \Gamma_{\xi}(\mathbf{P})+ \\
& \sum_{\mathrm{i}=1}^{\left(\mathrm{N}_{\mathrm{c}}-1\right)} \mathbf{R}_{\mathrm{ci}} *(\mathbf{Q}, \mathbf{P}) \mathbf{w}_{\mathrm{ci}}(\mathbf{P})+\lim _{\xi \rightarrow 0}\left[\mathbf{R}_{\mathrm{ci}^{-}} *(\mathbf{Q}, \mathbf{P}) \mathbf{w}_{\mathrm{ci}^{-} \mathrm{i}}(\mathbf{P})+\mathbf{R}_{\mathrm{ci}^{+}} *(\mathbf{Q}, \mathbf{P}) \mathbf{w}_{\mathrm{ci}^{+}}(\mathbf{P})\right]= \\
& \lim _{\bar{\Gamma} \rightarrow 0} \int_{\Gamma-\bar{\Gamma}}\left(V_{n}(\mathbf{Q}, \mathbf{P}) \mathbf{w} *(\mathbf{P})-\mathbf{m}_{\mathbf{n}}(\mathbf{Q}, \mathbf{P}) \mathbf{w},,_{\mathbf{n}} *(\mathbf{P})\right) \mathrm{d} \Gamma(\mathbf{P})+ \\
& \lim _{\xi \rightarrow 0} \int_{\Gamma_{\xi}}\left(V_{n}(\mathbf{Q}, \mathbf{P}) \mathbf{w} *(\mathbf{P})-\mathbf{m}_{\mathbf{n}}(\mathbf{Q}, \mathbf{P}) \mathbf{w},{ }_{\mathbf{n}}{ }^{*}(\mathbf{P})\right) \mathrm{d} \Gamma_{\xi}(\mathbf{P})+ \\
& \sum_{\mathrm{i}=1}^{\left(\mathrm{N}_{\mathrm{c}}-1\right)} \mathbf{R}_{\mathrm{ci}}(\mathbf{Q}, \mathbf{P}) \mathbf{w}_{\mathrm{ci}} *(\mathbf{P})+\lim _{\xi \rightarrow 0}\left[\mathbf{R}_{\mathrm{ci}^{-}}(\mathbf{Q}, \mathbf{P}) \mathbf{w}_{\mathrm{ci}^{-}} *(\mathbf{P})+\mathbf{R}_{\mathrm{ci}^{+}}(\mathbf{Q}, \mathbf{P}) \mathbf{w}_{\mathrm{ci}^{+}} *(\mathbf{P})\right]+ \\
& \int_{\Omega g} g(p) w *(Q, p) d \Omega_{g}(p)
\end{aligned}
$$

Os limites da integral $\Gamma_{\xi}$ que envolve os termos $\mathbf{w}(\mathbf{P})$ e $\mathbf{w}$, podem ser reescritos na forma:

$$
\begin{aligned}
& \lim _{\xi \rightarrow 0} \int\left[V_{\Gamma_{\xi}}\left[V_{n}^{*}(\mathbf{Q}, \mathbf{P}) \mathbf{w}(\mathbf{P})-\mathbf{m}_{\mathbf{n}}^{*} \frac{\partial \mathbf{w}}{\partial \mathbf{n}}(\mathbf{P})\right] \mathrm{d} \Gamma_{\xi}(\mathbf{P})=\right. \\
& \lim _{\xi \rightarrow 0} \int_{\Gamma_{\xi}}\left\{\mathbf{V}_{\mathbf{n}}^{*}(\mathbf{Q}, \mathbf{P})[\mathbf{w}(\mathbf{P})-\mathbf{w}(\mathbf{Q})]-\mathbf{m}_{\mathbf{n}}^{*}\left[\frac{\partial \mathbf{w}}{\partial \mathbf{n}}(\mathbf{P})-\frac{\partial \mathbf{w}}{\partial \mathbf{n}}(\mathbf{Q})\right]\right\} \mathrm{d} \Gamma_{\xi}(\mathbf{P})= \\
& \lim _{\xi \rightarrow 0} \int_{\Gamma_{\xi}}\left[\mathbf{V}_{\mathbf{n}}^{*}(\mathbf{Q}, \mathbf{P}) \mathbf{w}(\mathbf{Q})\right] \mathrm{d} \Gamma_{\xi}(\mathbf{P})-\lim _{\xi \rightarrow 0} \iint_{\Gamma_{\xi}}\left[\mathbf{m}_{\mathbf{n}}^{*} \frac{\partial \mathbf{w}}{\partial \mathbf{n}}(\mathbf{Q})\right] \mathrm{d} \Gamma_{\xi}(\mathbf{P})
\end{aligned}
$$

cuja continuidade permite afirmar que, quando $\xi \rightarrow 0$ o ponto $\mathbf{P} \rightarrow \mathbf{Q}$, e os valores $\mathbf{w}(\mathbf{Q})$ e $\mathbf{w},{ }_{n}(\mathbf{Q})$ não variam sobre $\Gamma_{\xi}$ : 


$$
\begin{aligned}
& \lim _{\xi \rightarrow 0} \int_{\Gamma_{\xi}}\left(V_{n} *(\mathbf{Q}, \mathbf{P}) \mathbf{w}(\mathbf{P})-\mathbf{m}_{\mathbf{n}} *(\mathbf{Q}, \mathbf{P}) \mathbf{w},{ }_{\mathbf{n}}(\mathbf{P})\right) \mathrm{d} \Gamma_{\xi}(\mathbf{P})= \\
& \mathbf{w}(\mathbf{Q}) \lim _{\xi \rightarrow 0} \int_{\Gamma_{\xi}} V_{n} *(\mathbf{Q}, \mathbf{P}) \mathbf{d} \Gamma_{\xi}(\mathbf{P})-\mathbf{w},{ }_{n}(\mathbf{Q}) \lim _{\xi \rightarrow 0} \int_{\Gamma_{\xi}} m_{n} *(\mathbf{Q}, \mathbf{P}) \mathbf{d} \Gamma_{\xi}(\mathbf{P})
\end{aligned}
$$

Substituindo-se $\mathbf{V}_{\mathrm{n}}^{*}$ e $\mathbf{m}_{\mathrm{n}}{ }^{*}$ na eq.(2.41), lembrando-se que $\mathbf{r}_{\mathrm{i}} \mathbf{n}_{\mathrm{i}}=\mathbf{r}_{\mathrm{n}}=1$, $\mathbf{r}_{, i} \mathbf{S}_{\mathrm{i}}=\mathbf{r}_{, \mathrm{s}}=0, \mathbf{r}=\boldsymbol{\xi} \mathrm{ed} \Gamma_{\xi}(\mathbf{P})=\boldsymbol{\xi} \mathrm{d} \boldsymbol{\theta}$ para $0=\boldsymbol{\theta}=\left(2 \pi-\boldsymbol{\beta}_{\mathrm{c}}\right)$, obtém-se:

$$
\lim _{\xi \rightarrow 0} \int_{\Gamma_{\xi}}\left(V_{n} *(Q, P) w(P)-m_{n} *(Q, P) w, n(P)\right) d \Gamma_{\xi}(P)=-\frac{2 \pi-\beta_{c}}{2 \pi} \mathbf{w}(\mathbf{Q})
$$

sendo $\boldsymbol{\beta}_{\mathrm{c}}$ o ângulo interno em $\mathbf{Q}$.

Os limites de integração sobre $\Gamma_{\xi}$ restantes na eq.(2.40), se analisados da mesma forma que até então, tenderão a zero se $\xi \rightarrow 0$, e portanto, também os limites envolvendo $\mathbf{R}_{\mathrm{c}}$.

Finalmente, a seguinte equação para o cálculo dos valores num ponto do contorno é obtida:

$$
\begin{aligned}
& \frac{\beta_{\mathrm{c}}}{2 \pi} \mathbf{w}(\mathbf{Q})+\int_{\Gamma}\left(\mathbf{V}_{\mathbf{n}} *(\mathbf{Q}, \mathbf{P}) \mathbf{w}(\mathbf{P})-\mathbf{m}_{\mathbf{n}} *(\mathbf{Q}, \mathbf{P}) \mathbf{w}, \mathbf{n}(\mathbf{P})\right) \mathbf{d} \Gamma(\mathbf{P})+ \\
& \sum_{\mathrm{i}=1}^{\mathrm{N}_{\mathrm{c}}} \mathbf{R}_{\mathrm{ci}} *(\mathbf{Q}, \mathbf{P}) \mathbf{w}_{\mathrm{ci}}(\mathbf{P})= \\
& \int_{\Gamma}\left(\mathbf{V}_{\mathbf{n}}(\mathbf{Q}, \mathbf{P}) \mathbf{w} *(\mathbf{P})-\mathbf{m}_{\mathbf{n}}(\mathbf{Q}, \mathbf{P}) \mathbf{w},,_{\mathbf{n}} *(\mathbf{P})\right) \mathrm{d} \Gamma(\mathbf{P})+ \\
& \sum_{\mathrm{i}=1}^{\mathrm{N}_{\mathrm{c}}} \mathbf{R}_{\mathrm{ci}}(\mathbf{Q}, \mathbf{P}) \mathbf{w}_{\mathrm{ci}} *(\mathbf{P})+\int_{\Omega \mathrm{g}} \mathrm{g}(\mathbf{p}) \mathbf{w} *(\mathbf{Q}, \mathbf{p}) \mathrm{d} \Omega_{\mathrm{g}}(\mathbf{p})
\end{aligned}
$$

Pode-se consultar a equação para o cálculo da derivada de $\mathbf{w}(\mathbf{Q})$ em relação a uma direção qualquer $\underline{\mathbf{m}}$ na bibliografia sobre o assunto (PAIVA(1987), p.e.). 


\subsection{3 - INTEGRAIS DE DOMÍNIO}

Conforme se vê nas eqs.(2.38 e 2.43) há uma integral de domínio que corresponde a uma carga distribuída aplicada sobre uma região $\boldsymbol{\Omega}_{\mathrm{g}}$ da placa, cujo contorno é $\Gamma_{\mathrm{g}}$. No MEC, entretanto, é conveniente a transformação desta integral sobre o domínio em integral sobre o contorno $\left(\boldsymbol{\Gamma}_{\mathrm{g}}\right)$. Desta forma, facilita-se a sua resolução numérica pela divisão do contorno da região carregada em elementos, embora não se refira a variáveis incógnitas. Entretanto, conforme será visto adiante, para possibilitar a vinculação em pontos internos da placa a pontos de barras, é interessante também que se faça distinção do tipo e da geometria do carregamento quando da elaboração desta integral de contorno.

\subsection{1 - CARGAS DISTRIBUÍDAS EM REGIÕES DA PLACA}

Algumas técnicas são usadas para a migração das variáveis da integral de $\boldsymbol{\Omega}_{\mathrm{g}}$ para $\Gamma_{\mathrm{g}}$. Aqui, será feita seguindo o seguinte procedimento: observando-se a fig.(2.7) pode-se escrever que

$$
d \Omega_{g}=\mathbf{r d r} d \theta
$$

e

$$
\mathbf{d} \theta \frac{\mathbf{R}}{\cos \beta}=\mathbf{d} \Gamma_{\mathrm{g}} \quad \text { onde } \cos \boldsymbol{\beta}=\mathbf{r}_{\mathrm{n}}=\mathbf{r}_{\mathrm{i}} \mathbf{n}_{\mathrm{i}}
$$

Portanto

$$
d \Omega_{g}=r d r \frac{r,{ }_{n}}{R} d \Gamma_{g}
$$




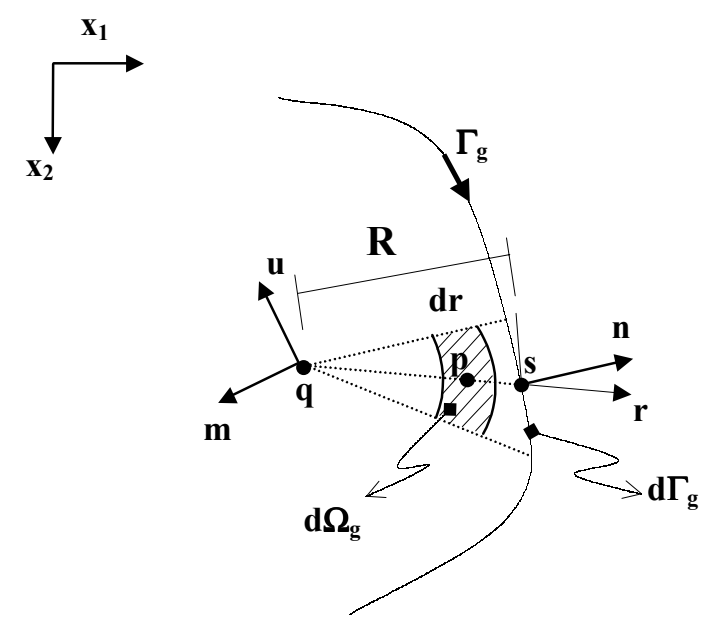

Fig. 2.7 - Domínio da região do carregamento

que transforma a integral de domínio em

$$
\int_{\Omega g} g(p) w *(Q, p) d \Omega_{g}(p)=\int_{\Gamma g 0}^{R} \int_{g} g(p) w *(Q, p) r d r \frac{r, g_{n}}{R} d \Gamma_{g}(p)
$$

Antes de proceder a integração acima, deve-se estabelecer a variação da carga sobre o sistema de coordenadas $\mathbf{x}_{1} \mathbf{x}_{2}$. Conforme CHUEIRI(1994), pode-se, por exemplo, supor que seja linear esta variação, ou seja:

$$
\mathbf{g}(\mathbf{p})=\mathbf{A} \mathbf{x}_{1}(\mathbf{p})+\mathbf{B} \mathbf{x}_{2}(\mathbf{p})+\mathbf{C}
$$

e em termos de $\mathbf{r}$ e $\boldsymbol{\theta}$ e sendo $\mathbf{A}, \mathbf{B}$ e $\mathbf{C}$ constantes e

$$
\begin{aligned}
& \mathbf{x}_{1}(\mathbf{p})=\mathbf{x}_{1}(\mathbf{q})+r \cos \theta \\
& \mathbf{x}_{2}(\mathbf{p})=\mathbf{x}_{2}(\mathbf{q})+r \sin \theta
\end{aligned}
$$

chega-se a uma variação de $\mathbf{g}(\mathbf{p})$ que é

$$
g(p)=A r \cos \theta+B r \sin \theta+g(q)
$$

uma vez que

$$
\mathbf{g}(\mathbf{q})=\mathbf{A} \mathbf{x}_{1}(\mathbf{q})+\mathbf{B} \mathbf{x}_{2}(\mathbf{q})+\mathbf{C} \quad \text { é um valor constante. }
$$

Substituindo-se eq.(2.45) e w* (eq.(2.20)) na eq.(2.44), calcula-se a integração sobre $\mathbf{r}$. Então, a integral de domínio se torna de contorno que vale: 


$$
\begin{aligned}
& \int_{\Omega g} g(p) w *(Q, p) d \Omega_{g}(p)=\frac{g(q)}{32 \pi D} \int_{\Gamma g} R^{3}\left(\ln R-\frac{3}{4}\right) r,{ }_{n} d \Gamma_{g}(p)+ \\
& \frac{1}{40 \pi D} \int_{\Gamma g} R^{4}\left(\ln R-\frac{7}{10}\right)(A \cos \theta+B \sin \theta) r,{ }_{n} d \Gamma_{g}(p)
\end{aligned}
$$

\subsection{2 - CARGAS DISTRIBUÍDAS EM LINHAS OU CARGAS EM REGIÕES DISCRETAS}

A eq.(2.46), permite o cálculo de deslocamentos e esforços no contorno e domínio de uma placa sob carregamento distribuído em regiões do domínio da placa e com o conhecimento de suas condições de contorno.

Existe o interesse, porém, de se analisar placas com condições de vinculação no seu domínio. Estas condições podem ter sua influência sobre a rigidez da placa considerada, se os esforços de interface forem interpretados como carregamento distribuído em pequenas regiões, como linhas de carga $\left(\mathbf{S}_{\mathbf{l i}}\right)$ ou cargas distribuídas em regiões discretas $\left(\mathbf{S}_{\mathbf{d i}}\right)$.

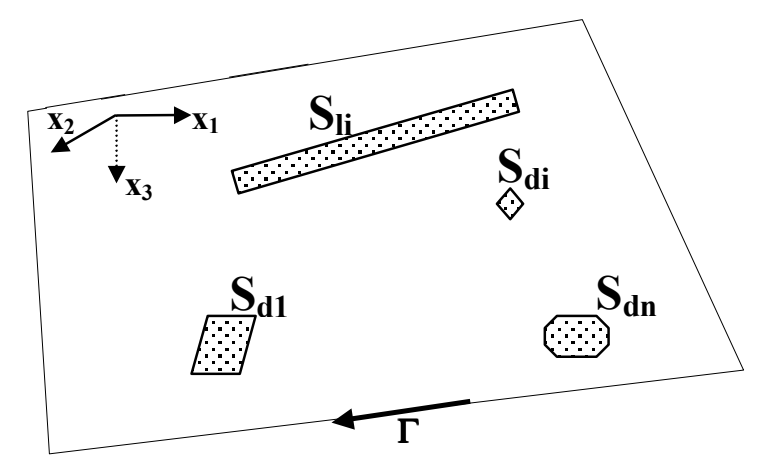

Fig. 2.8 - Carregamento discreto ou em linha

Daí, a parcela da integral de domínio da eq.(2.38), por exemplo, referente a esta influência pode ser:

$$
I_{q}=\sum_{i=1}^{n l} \int_{S l i} g_{l i} w^{*}(Q, p) d S_{l i}(p)+\sum_{i=1}^{n d} \int_{S d i} g_{d i} w^{*}(Q, p) d S_{d i}(p)
$$

para $\mathbf{n}_{\mathbf{l}}$ e $\mathbf{n}_{\mathbf{d}}$ representando o número de regiões com carregamento em linha ou em áreas discretas, respectivamente. Supondo-se as cargas sobre as áreas $\mathbf{S}_{\mathbf{l i}}$ (carga $\mathbf{g}_{\mathbf{l i}}$ ) e 
$\mathbf{S}_{\mathbf{d i}}$ (carga $\mathbf{g}_{\mathrm{di}}$ ) como uniformemente distribuídas, a integral pode ser matricialmente representada por:

$$
I_{q}=[S]\left\{P_{\Omega}\right\}
$$

Note-se que, quando os componentes de $\left\{\mathbf{P}_{\boldsymbol{\Omega}}\right\}$ são desconhecidos, irão aumentar o número de incógnitas do problema. Isto é resolvido escrevendo-se as representações integrais relativas ao deslocamento e à sua derivada (eqs. 2.38 e 2.39) para tantos quantos forem os nós da interface ou da linha de carga, considerando-se também a influência dos vínculos internos na integral de domínio. Neste caso, podem surgir, porém, problemas com singularidade nas integrais, pois os nós $\mathbf{p}$ e $\mathbf{q}$ coincidem. Estas integrais, embora para a placas tratadas pelas hipóteses de Reissner, se encontram excelentemente desenvolvidas por SILVA(1996), que também apresentou outras condições de carregamento possíveis no domínio da placa.

\subsection{4 - O MEC APLICADO À ANÁLISE DE PLACAS DELGADAS}

Para a aplicação do MEC ao problema de flexão de placas utilizando-se a formulação integral apresentada, é precisa dividir-se o seu contorno em elementos, cujo valor das variáveis $\mathbf{m}_{\mathrm{n}}, \mathbf{V}_{\mathrm{n}}$, $\mathbf{w}$ e $\mathbf{w}_{\mathrm{n}}$ sobre eles seja calculado por funções aproximadoras dos valores destas variáveis nos nós extremos dos elementos. A integral sobre todo o contorno da placa, portanto, é substituído pela soma das integrações sobre o contorno de cada elemento. Daí, a equação integral se transforma numa equação algébrica envolvendo valores nodais daquelas variáveis no contorno.

Escrevendo-se equações para os deslocamentos dos nós de contorno da placa, constrói-se um sistema de equações lineares onde os valores incógnitos são os deslocamentos e esforços nos pontos do contorno. A imposição das condições de contorno, portanto, permite que se resolva o sistema de equações para os valores destas incógnitas. Isto permite com que se calculem outros valores em qualquer ponto do domínio da placa.

Como se sabe, associam-se a cada ponto do contorno da placa, quatro variáveis, duas das quais são conhecidas através das condições de contorno. Necessitam-se então, duas equações por nó para resolver os sistema. Há autores que preferem uma equação para os deslocamentos lineares $\mathbf{w}$ e outra para a rotação $\theta_{\mathrm{n}}$ 
(PAIVA(1987), WEARING(1994)). Outros utilizaram, como CHUEIRI(1994), uma técnica alternativa que estabelece uma equação do deslocamento linear $\mathbf{w}$ para cada ponto e outra para um ponto externo a ele associado. CHUEIRI(1994) também utilizou as reações dos cantos das placas e seus respectivos deslocamentos como variáveis do problema, escrevendo equações de deslocamentos para estes pontos e utilizando as condições de contorno a eles equivalentes, para a resolução do sistema.

Portanto, um algoritmo foi desenvolvido com base na teoria aqui estudada. Neste algoritmo, os elementos de contorno utilizados tiveram a sua geometria aproximada por funções lineares e a distribuição das variáveis sobre eles aproximadas por funções quadráticas.

Como foi visto (ver eq. 2.43), a forma geral da equação integral para o cálculo do deslocamento de um ponto $\mathbf{Q}$ do contorno de uma placa delgadas é:

$$
\begin{aligned}
& \mathrm{C}(\mathrm{Q}) \mathrm{w}(\mathrm{Q})+\int_{\Gamma}\left(\mathrm{V}_{\mathrm{n}} *(\mathrm{Q}, \mathrm{P}) \mathrm{w}(\mathrm{P})-\mathrm{m}_{\mathrm{n}} *(\mathrm{Q}, \mathrm{P}) \mathrm{w},{ }_{\mathrm{n}}(\mathrm{P})\right) \mathrm{d} \Gamma(\mathrm{P})+ \\
& \sum_{i=1}^{N_{c}} R_{c i} *(Q, P) w_{c i}(P)= \\
& \int_{\Gamma}\left(V_{n}(P) w *(Q, P)-m_{n}(P) w,,_{n}^{*}(Q, P)\right) d \Gamma(P)+ \\
& \sum_{i=1}^{N_{c}} R_{c i}(P) w_{c i} *(Q, P)+\int_{\Omega g} g(p) w *(Q, p) d \Omega_{g}(p)
\end{aligned}
$$

que envolve os valores fundamentais e depende das variáveis de todo o contorno. $\mathrm{Na}$ eq.(2.49) $\mathbf{C}(Q)$ vale:

$$
C(Q)=\frac{\beta_{c}}{2 \pi}
$$

sendo $\boldsymbol{\beta}_{\mathbf{c}}$ o ângulo interno em $\mathbf{Q}$. Note-se que, quando $\mathbf{Q}$ está num contorno sem angulosidade $\left(\boldsymbol{\beta}_{\mathbf{c}}=\boldsymbol{\pi}\right), \mathbf{C}(\mathbf{Q})=\mathbf{1} / \mathbf{2}$; quando $\mathbf{Q}$ é um ponto interno $(\mathbf{q})$ pertencente ao domínio da placa, então $\mathbf{C}(\mathbf{Q})=\mathbf{1}$ e $\mathbf{C}(\mathbf{Q})=\mathbf{0}$ em caso contrário.

CHUEIRI (1994) simplifica a eq.(2.49) definindo o vetor de deslocamentos e o de seus valores fundamentais: 


$$
\begin{aligned}
& \underset{\sim}{\mathbf{u}}(\mathbf{P})=\left\{\begin{array}{l}
\mathbf{u}_{1}(\mathbf{P}) \\
\mathbf{u}_{2}(\mathbf{P})
\end{array}\right\}=\left\{\begin{array}{c}
\mathbf{w}(\mathbf{P}) \\
\mathbf{w}, \mathbf{n}(\mathbf{P})
\end{array}\right\} \\
& \underset{\sim}{\mathbf{u}^{*}}(\mathbf{Q}, \mathbf{P})=\left[\mathbf{w} *(\mathbf{Q}, \mathbf{P}) \quad-\mathbf{w},,_{\mathbf{n}}^{*}(\mathbf{Q}, \mathbf{P})\right] \\
& \mathbf{u}_{\mathrm{g}} *(\mathbf{Q}, \mathbf{p})=\mathbf{w} *(\mathbf{Q}, \mathbf{p}) \\
& \mathbf{u}(\mathbf{Q})=\mathbf{w}(\mathbf{Q})
\end{aligned}
$$

e mais o vetor das forças de superfície e o de seus valores fundamentais:

$$
\begin{aligned}
& \underset{\sim}{\mathbf{p}(P)}=\left\{\begin{array}{l}
\mathbf{p}_{1}(\mathbf{P}) \\
\mathbf{p}_{2}(\mathbf{P})
\end{array}\right\}=\left\{\begin{array}{c}
\mathbf{V}_{\mathbf{n}}(\mathbf{P}) \\
\mathbf{m}_{\mathrm{n}}(\mathbf{P})
\end{array}\right\} \\
& \underset{\sim}{\mathbf{p}}(\mathbf{Q}, \mathbf{P})=\left[\mathbf{V}_{\mathbf{n}} *(\mathbf{Q}, \mathbf{P}) \quad-\mathbf{m}_{\mathbf{n}} *(\mathbf{Q}, \mathbf{P})\right]
\end{aligned}
$$

Ao mesmo tempo, como foi dito, torna-se necessário dividir-se o contorno da placa em $\mathbf{N}_{\mathrm{e}}$ elementos para discretizar a integral eq.(2.49). Este processo de discretização permite que se transforme a equação integral geral sobre todo o contorno em uma somatória das integrais sobre o contorno de cada elemento $\mathbf{j}$, cujo contorno é $\Gamma_{\mathbf{j}}$. Portanto, a eq.(2.49) fica:

$$
\begin{aligned}
& \mathbf{C}(\mathbf{Q}) \mathbf{w}(\mathbf{Q})+\sum_{\mathrm{j}=1}^{\mathrm{N}_{\mathrm{e}}} \int_{\Gamma_{\mathrm{j}}}\left(\mathbf{p}^{*}(\mathbf{Q}, \mathbf{P}) \cdot \underset{\sim}{\mathbf{u}}(\mathbf{P}) \mathbf{d} \Gamma_{\mathrm{j}}(\mathbf{P})+\sum_{\mathrm{i}=1}^{\mathbf{N}_{\mathrm{c}}} \mathbf{R}_{\mathrm{ci}} *(\mathbf{Q}, \mathbf{P}) \mathbf{w}_{\mathrm{ci}}(\mathbf{P})=\right. \\
& \sum_{\mathbf{j}=1}^{N_{\mathrm{e}}} \int_{\Gamma_{\mathbf{j}}}\left(\mathbf{p}(\mathbf{P}) \cdot \underset{\sim}{\mathbf{u}^{*}}(\mathbf{Q}, \mathbf{P}) \mathbf{d} \Gamma_{\mathbf{j}}(\mathbf{P})+\right. \\
& \sum_{i=1}^{N_{c}} R_{c i}(P) w_{c i} *(Q, P)+\int_{\Omega g} g(p) u_{g} *(Q, p) d \Omega_{g}(p)
\end{aligned}
$$

A vantagem desta divisão do contorno da placa em elementos é também o fato de que a variável relativa a um ponto genérico $\underline{\mathbf{P}}$ pode ser escrita como uma interpolação de seus valores em pontos do elemento pré-estabelecidos, os chamados valores nodais. Isto é possível pois no MEC assume-se que as funções de forma da geometria de cada elemento e de suas variáveis são conhecidas, o que permite-se escrever funções de interpolação $\phi$ que as represente. Neste trabalho, como o 
programa utilizado como base dos seus objetivos (CHUEIRI(1994)) assume funções polinomiais linear para geometria e quadrática para as variáveis (formulação superparamétrica), esta será a aproximação aqui adotada.

Então, definindo-se o vetor valores nodais de deslocamento e esforços de um elemento como $\mathbf{U}^{\mathbf{N}}$ e $\mathbf{P}^{\mathbf{N}}$, respectivamente, de forma que:

$$
\begin{aligned}
& \underset{\sim}{\mathbf{U}^{\mathbf{N}}}=\left\{\mathbf{U}_{\mathbf{i}}^{\mathbf{N}}\right\}=\left\{\begin{array}{c}
\mathbf{U}_{1}^{\mathbf{N}} \\
\mathbf{U}_{2}^{\mathbf{N}}
\end{array}\right\}=\left\{\begin{array}{c}
\mathbf{w}^{\mathbf{N}} \\
\mathbf{w},{ }_{n}^{N}
\end{array}\right\} \\
& {\underset{\sim}{\mathbf{N}}}^{\mathbf{N}}=\left\{\mathbf{P}_{i}^{\mathbf{N}}\right\}=\left\{\begin{array}{l}
\mathbf{P}_{1}^{\mathbf{N}} \\
\mathbf{P}_{2}^{\mathbf{N}}
\end{array}\right\}=\left\{\begin{array}{c}
\mathbf{V}^{\mathbf{N}} \\
\mathbf{m}_{\mathbf{n}}^{\mathbf{N}}
\end{array}\right\}
\end{aligned}
$$

sendo que $\underline{\mathbf{N}}$ representa o número do ponto do elemento de contorno que varia de 1 a 3 já que o elemento escolhido foi o quadrático, conforme já comentado anteriormente. Pode-se dizer sobre um ponto genérico $\underline{\mathbf{P}}$ deste elemento que:

$$
\begin{aligned}
& \underset{\sim}{\mathbf{u}}(\mathbf{P})=\left\{\begin{array}{l}
\mathbf{u}_{1}(\mathbf{P}) \\
\mathbf{u}_{2}(\mathbf{P})
\end{array}\right\}=\left\{\begin{array}{c}
\mathbf{w}(\mathbf{P}) \\
\mathbf{w},{ }_{\mathrm{n}}(\mathbf{P})
\end{array}\right\}={\underset{\sim}{\Phi}}^{\mathrm{T}}(\mathbf{P}) \cdot{\underset{\sim}{\mathbf{U}^{\mathrm{N}}}}^{\mathrm{N}} \\
& \underset{\sim}{\mathbf{p}(\mathbf{P})}=\left\{\begin{array}{l}
\mathbf{p}_{1}(\mathbf{P}) \\
\mathbf{p}_{2}(\mathbf{P})
\end{array}\right\}=\left\{\begin{array}{c}
\mathbf{V}_{\mathbf{n}}(\mathbf{P}) \\
\mathbf{m}_{\mathbf{n}}(\mathbf{P})
\end{array}\right\}={\underset{\sim}{\Phi}}^{\mathbf{T}}(\mathbf{P}) \cdot{\underset{\sim}{\mathbf{P}}}^{\mathbf{N}}
\end{aligned}
$$

onde

$$
\underset{\sim}{\Phi}=\left[\begin{array}{cccccc}
\phi_{1}(\mathbf{P}) & \mathbf{0} & \phi_{2}(\mathbf{P}) & \mathbf{0} & \phi_{3}(\mathbf{P}) & \mathbf{0} \\
\mathbf{0} & \phi_{1}(\mathbf{P}) & \mathbf{0} & \phi_{2}(\mathbf{P}) & \mathbf{0} & \phi_{3}(\mathbf{P})
\end{array}\right]
$$

e $\phi_{i}$ são as funções interpolação quadrática.

Considerando-se $\xi$ a coordenada local homogênea, cuja vantagem de sua utilização será discutida adiante, as funções $\phi_{\mathrm{i}}$ são escritas: 


$$
\begin{aligned}
& \phi_{1}(\mathbf{P})=\frac{\left(\xi_{3}-\xi\right) \xi}{\xi_{1}\left(\xi_{3}-\xi_{1}\right)} \\
& \phi_{2}(\mathbf{P})=1-\frac{\left(\xi_{3}+\xi_{1}\right) \xi}{\xi_{1} \xi_{3}}+\frac{\xi^{2}}{\xi_{1} \xi_{3}} \\
& \phi_{3}(\mathbf{P})=\frac{\left(\xi_{1}-\xi\right) \xi}{\xi_{3}\left(\xi_{1}-\xi_{3}\right)}
\end{aligned}
$$

O formato das equações $\mathbf{2 . 5 6}$ considera a possibilidade da existência de um ou ambos pontos extremos do elementos com dois valores nodais da mesma variável cada um. Daí, pontos com esta particular condição, deve ser migrados para dentro do comprimento do elemento (elemento descontínuo). Caso isto não ocorra, o nó deve ser mantido na sua posição original as coordenadas locais valerão $\xi_{1}=-1$ ou $\xi_{3}=+1$ nas eqs.(2.56), dependendo da sua posição no elemento in. Se ambos nós extremos do elemento de contorno possuem um único valor para as variáveis a eles associadas, obviamente que ambos serão mantidos em suas posições originais caracterizando assim, um elemento chamado contínuo.

Reescreve-se a eq.(2.52) considerando-se as eqs.(2.54) na forma:

$$
\begin{aligned}
& \mathbf{C}(\mathbf{Q}) \mathbf{u}(\mathbf{Q})+\sum_{\mathrm{j}=1}^{\mathrm{N}_{\mathrm{e}}}\left[\underset{\sim \mathbf{j}}{\mathbf{h}}(\mathbf{Q}) \cdot \underset{\sim \mathrm{j}}{\mathbf{U}^{\mathrm{N}}}\right]+\sum_{\mathrm{i}=1}^{\mathrm{N}_{\mathrm{c}}} \mathbf{R}_{\mathrm{ci}} *(\mathbf{Q}, \mathbf{P}) \mathbf{w}_{\mathrm{ci}}(\mathbf{P})= \\
& \sum_{j=1}^{N_{e}}\left[\underset{\sim j}{g}(Q) \cdot{\underset{\sim j}{j}}_{\mathbf{P}^{\mathrm{N}}}^{\mathbf{g}}\right]+\sum_{\mathrm{i}=1}^{\mathbf{N}_{\mathrm{c}}} \mathbf{R}_{\mathrm{ci}}(\mathbf{P}) \mathbf{w}_{\mathrm{ci}} *(\mathbf{Q}, \mathbf{P})+\mathbf{t}(\mathbf{Q})
\end{aligned}
$$

onde

$$
\begin{aligned}
& \underset{\sim j}{\mathbf{h}}(Q)=\int_{\Gamma_{j}}\left[\mathbf{p}_{\sim}^{*}(Q, P) \cdot \underset{\sim}{\Phi^{T}}(P)\right] d \Gamma_{j}(P) \\
& \underset{\sim j}{g}(Q)=\int_{\Gamma_{j}}\left[\underset{\sim}{\mathbf{u}_{j}}(Q, P) \cdot \underset{\sim}{\Phi^{T}}(P)\right] d \Gamma_{j}(P) \\
& t(Q)=\int_{\Omega_{\mathrm{g}}}\left[g(p) \cdot \mathbf{u}^{*}(Q, p)\right] d \Omega_{g}(p)
\end{aligned}
$$

cujos valores das integrais para cada elemento $\mathbf{j}$ são conhecidos e são multiplicados pelos valores nodais $\mathbf{U}_{\mathbf{j}}^{\mathbf{N}}$ e $\mathbf{P}_{\mathbf{j}}^{\mathbf{N}}$. Esta é a vantagem em se utilizar o sistema local de 
coordenadas, o que consiste em se considerar as coordenadas de cada ponto do elemento como uma coordenada local. Então, as integrais sobre o elemento podem ser numericamente resolvidas.

Após somar-se a influência de cada elemento de contorno e dos cantos no cálculo do deslocamento de um determinado ponto $\mathbf{Q}$, os valores nodais multiplicados pelos seus coeficientes são agrupados, construindo-se assim, uma linha de uma matriz. Procedendo da mesma forma para todos os nós do contorno da placa, incluindo os cantos, obtém-se a seguinte forma matricial para a eq.(2.57):

$$
\mathbf{C}(\mathbf{Q}) \mathbf{u}(\mathbf{Q})+\underset{\sim}{\mathbf{H}}(\mathbf{Q}) \underset{\sim}{\mathbf{U}}+\underset{\sim c}{\mathbf{H}}(\mathbf{Q}) \underset{\sim c}{\mathbf{w}}=\underset{\sim}{\mathbf{G}}(\mathbf{Q}) \underset{\sim}{\mathbf{P}}+\underset{\sim \mathrm{c}}{\mathbf{G}}(\mathbf{Q}) \underset{\sim \mathrm{c}}{\mathbf{R}}+\mathbf{T}(\mathbf{Q})
$$

onde

$\rightarrow \underset{\sim}{\hat{H}}(\mathbf{Q})$ e $\underset{\sim}{\mathbf{G}(\mathbf{Q})}$ contêm os valores das duas primeiras das eqs.(2.58), respectivamente, agrupadas de acordo com os valores nodais a que se relacionam;

$\rightarrow \underset{\sim c}{\mathbf{H}}(\mathbf{Q})$ e $\underset{\sim \mathbf{c}}{\mathbf{G}}(\mathbf{Q})$ contêm os coeficientes que multiplicam os deslocamentos e reações nos cantos, respectivamente;

$\rightarrow \mathbf{T}(\mathbf{Q})$ refere-se aos valores calculados na última das eqs.(2.58);

$$
\begin{aligned}
& \rightarrow \underset{\sim}{\mathbf{U}^{T}}=\left\{\begin{array}{lllll}
\mathbf{W}^{1} & \mathbf{W},{ }_{\mathbf{n}}^{1} & \ldots & \mathbf{W}^{\mathbf{N}_{\mathbf{n}}} & \mathbf{W},,_{\mathbf{n}}^{\mathbf{N}_{\mathbf{n}}}
\end{array}\right\} \\
& \rightarrow \underset{\sim}{\mathbf{P}^{T}}=\left\{\begin{array}{lllll}
\mathbf{V}_{\mathbf{n}}^{1} & \mathbf{m}_{\mathbf{n}}^{1} & \ldots & \mathbf{V}_{\mathbf{n}}^{\mathbf{N}_{\mathbf{n}}} & \mathbf{m}_{\mathbf{n}}^{\mathbf{N}_{\mathbf{n}}}
\end{array}\right\}
\end{aligned}
$$

sendo $\mathbf{N}_{\mathrm{n}}$ o número de pontos do contorno e

$$
\begin{aligned}
& \rightarrow \underset{\sim c}{\mathbf{w}_{\mathbf{c}}^{\mathbf{T}}}=\left\{\begin{array}{llll}
\mathbf{W}_{\mathbf{c}_{1}} & \mathbf{w}_{\mathbf{c}_{2}} & \cdots & \mathbf{w}_{\mathbf{c}^{\mathrm{Nc}}}
\end{array}\right\} \\
& \rightarrow \underset{\sim \mathbf{c}}{\mathbf{R}}=\left\{\begin{array}{llll}
\mathbf{R}_{\mathbf{c}_{1}} & \mathbf{R}_{\mathbf{c}_{2}} & \cdots & \mathbf{R}_{\mathrm{c}^{\mathrm{Nc}}}
\end{array}\right\}
\end{aligned}
$$

Aqui, $\mathbf{N}_{\mathrm{c}}$ é o número de cantos da placa. 
Escrevendo-se a eq.(2.59) indicialmente, obtém-se uma linha (i) do sistema de equações:

$$
C^{i} u^{i}+\hat{H}{ }^{i j} U^{j}+H_{c} w_{c}=G^{i j} P^{j}+G_{c} R_{c}+T^{i s}
$$

onde

$$
\begin{array}{ll}
\mathbf{H}^{\mathrm{ij}}=\hat{\mathbf{H}}^{\mathrm{ij}} & \text { quando } \mathrm{i} \neq \mathrm{j} . \\
\mathbf{H}^{\mathrm{ij}}=\mathbf{C}^{\mathbf{i}}+\hat{\mathbf{H}}^{\mathrm{ij}} & \text { quando } \mathrm{i}=\mathrm{j} .
\end{array}
$$

Pode-se também escrever a eq.(2.60) como:

$$
\mathbf{H ~ U}+\mathbf{H}_{\mathbf{c}} \mathbf{w}_{\mathbf{c}}=\mathbf{G} \mathbf{P}+\mathbf{G}_{\mathbf{c}} \mathbf{R}_{\mathbf{c}}+\mathbf{T}
$$

A inclusão de $\mathbf{w}_{\mathrm{c}}$ e $\mathbf{R}_{\mathrm{c}}$ como variáveis do problema permite que se incorporem os termos em $\underset{\sim \mathbf{c}}{\mathbf{H}}(\mathbf{Q})$ e $\underset{\sim \mathbf{c}}{\mathbf{G}}(\mathbf{Q})$ em $\mathbf{H}(\mathbf{Q})$ e $\mathbf{G}(\mathbf{Q})$, transformando a eq.(2.61) na forma matricial:

$$
\underset{\sim}{\mathbf{H}}(\mathbf{Q}) \underset{\sim}{\mathbf{U}}=\underset{\sim}{\mathbf{G}}(\mathbf{Q}) \underset{\sim}{\mathbf{P}}+\mathbf{T}(\mathbf{Q})
$$

A partir dos resultados de deslocamentos e forças obtidos desta forma, podemse calcular os deslocamentos de qualquer ponto do contorno ou domínio da placa através das equações eqs. 2.38, 2.39 e 2.43. Para o cálculo de esforços e tensões e deformações nestes pontos, utilizam-se as equações dos itens 2.4 a $\mathbf{2 . 6}$ (ver, p.e., CHUEIRI(1994)). Para efeito de programação, dá-se a elas o mesmo tratamento dado à equação $\mathbf{2 . 4 3}$ que culminou na forma matricial do sistema em $\mathbf{2 . 6 2}$.

A matriz $\mathbf{H}$ dada em $\mathbf{2 . 6 2}$ é tal que possui propriedades que dizem respeito a configurações de equilíbrio de uma placa. Submetida a um carregamento nulo, podese escrever eq. $\mathbf{2 . 6 2}$ como:

$$
\underset{\sim}{\mathbf{H}}(\mathbf{Q}) \underset{\sim}{\mathbf{U}}=\mathbf{0}
$$

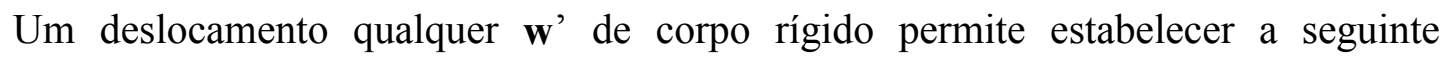
propriedade de $\mathbf{H}$ : 


$$
\sum_{j=1}^{N n} h_{i, 2 j-1}+\sum_{j=1}^{N n} h_{c_{i, j}}=0
$$

ou seja, a soma entre os valores das colunas ímpares de qualquer linha de $\underset{\sim}{\mathbf{H}}$ deve ser igual a zero.

Seguindo o mesmo raciocínio, agora para uma rotação $\alpha$ de corpo rígido em torno de um eixo qualquer, obtém-se a outra propriedade de $\mathbf{H}$ :

$$
\sum_{j=1}^{N n} h_{i, 2 j-1} \cdot D_{j}+\sum_{j=1}^{N n} h_{i, 2 j} \cdot \cos \beta_{j}+\sum_{j=1}^{N n} h_{i, j} \cdot D c_{j}=0
$$

onde $\mathbf{D}_{\mathbf{j}}$ é a distância do nó $\mathbf{j}$ ao eixo arbitrário de rotação e $\boldsymbol{\beta}_{\mathbf{j}}$ é o ângulo formado entre a normal ao contorno em $\mathbf{j}$ e o versor normal ao eixo em torno do qual a placa gira.

No problema de flexão de placas delgadas, como se sabe, quatro são as variáveis associadas a cada ponto do contorno, $\mathbf{w}(\mathbf{P}), \mathbf{w},{ }_{\mathbf{n}}(\mathbf{P}), \mathbf{V}_{\mathbf{n}}(\mathbf{P})$ e $\mathbf{m}_{\mathbf{n}}(\mathbf{P})$, duas das quais são determinadas pelas condições de contorno. Considerando-se todo o contorno da placa, restam $2 \mathbf{N}_{\mathbf{n}}$ valores desconhecidos, dois para cada nó. Além disto, com relação aos cantos, um dos dois valores associados a cada um deles, $\mathbf{w}_{\mathrm{c}}$ e $\mathbf{R}_{\mathrm{c}}$, novamente, é conhecido através das condições de apoio de cada canto. Restam, então, $\mathbf{N}_{\mathrm{c}}$ incógnitas por nó de canto.

Diante disso, resolve-se o problema de flexão de placas ao se escrever duas equações para cada nó do contorno e uma para cada canto através da eq.(2.59). O procedimento usual é se escrever uma equação integral para o derivada direcional do deslocamento $\mathbf{w}(\mathbf{Q})$ de cada ponto, relativa a uma direção genérica $\overrightarrow{\mathbf{m}}$, de forma que, da eq.(2.49): 


$$
\begin{aligned}
& \mathbf{C}(\mathbf{Q}) \mathbf{w},{ }_{\mathrm{m}}(\mathrm{Q})+\int_{\Gamma}\left[\mathrm{V}_{\mathrm{n}},_{\mathrm{m}} *(\mathrm{Q}, \mathrm{P}) \mathbf{w}(\mathrm{P})-\mathbf{m}_{\mathrm{n}},{ }_{\mathrm{m}} *(\mathrm{Q}, \mathrm{P}) \mathbf{w},,_{\mathrm{n}}(\mathrm{P})\right] \mathrm{d} \Gamma(\mathrm{P})+ \\
& \sum_{\mathrm{i}=1}^{\mathrm{N}_{\mathrm{c}}} \mathbf{R}_{\mathrm{ci}} \boldsymbol{\prime}_{\mathrm{m}} *(\mathbf{Q}, \mathbf{P}) \mathbf{w}_{\mathrm{ci}}(\mathbf{P})= \\
& \int_{\Gamma}\left[V_{n}(P) w,,_{m}^{*}(Q, P)-m_{n}(P) w,,_{n m} *(Q, P)\right] d \Gamma(P)+ \\
& \sum_{i=1}^{N_{c}} R_{c i}(P) w_{c i},_{m} *(Q, P)+\int_{\Omega g} g(p) w,,_{m} *(Q, p) d \Omega_{g}(p)
\end{aligned}
$$

Pode-se demonstrar que é possível transformar-se a eq.(2.63) em uma forma similar à eq.(2.62) procedendo-se da mesma maneira que ela foi obtida.

Porém, como já se mencionou, PAIVA(1987) e CHUEIRI(1994), dentre outros autores, utilizaram um artifício alternativo que consiste em escrever a segunda equação relacionada a um nó do contorno através da eq.(2.49), porém para um nó externo à placa a ele relacionado (Q') localizado fora do domínio da placa. VENTURINI (1989) e PAIVA \& VENTURINI (1992), dentre muitos outros autores, apresentam uma técnica de análise de flexão de placas através do MEC em que se evita a representação algébrica das rotações nos nós de contorno, através do uso de nós de colocação externos ao domínio, o que melhora os resultados obtidos. A definição da posição é extensamente estudada nos citados trabalhos. Estes pontos externos são posicionados na direção normal ao contorno no ponto em questão, a uma distância $\underline{\mathbf{d}}$ do mesmo, que é tal que (fig.2.9):

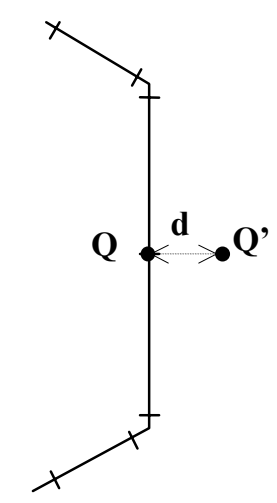

Fig. 2.9 - Ponto Externo Relativo ao do Contorno

$$
\mathbf{d}=\alpha \mathbf{l}_{\mathbf{m}}
$$


sendo $l_{\mathrm{m}}$ o valor médio entre os comprimentos dos elementos concorrentes neste ponto do contorno ou o comprimento do elemento, caso o nó em questão seja o nó central do elemento. Vários estudos existem com relação ao valor ideal para $\alpha$. CHUEIRI(1994) adotou $0.5 \leq \boldsymbol{\alpha} \leq 1.5$.

Pode-se escrever a forma matricial para a eq.(2.49) de forma a representar os deslocamentos de ambos os pontos $\mathbf{Q}$ e $\mathbf{Q}$ ':

$$
\begin{aligned}
& \underset{\sim}{\mathbf{C}}(\mathbf{Q}) \underset{\sim}{\mathbf{u}}(\mathbf{Q})+\int_{\Gamma}\left(\mathbf{p}_{\sim}^{*}(\mathbf{Q}, \mathbf{P}) \cdot \underset{\sim}{\mathbf{u}}(\mathbf{P}) \mathrm{d} \Gamma(\mathbf{P})+\sum_{\mathrm{i}=1}^{\mathbf{N}_{\mathrm{c}}} \underset{\sim \mathrm{ci}}{\mathbf{p}}{ }^{*}(\mathbf{Q}, \mathbf{P}) \mathbf{w}_{\mathrm{ci}}(\mathbf{P})=\right. \\
& \int_{\Gamma}(\mathbf{p}(\mathbf{P}) \cdot \underset{\sim}{\mathbf{u}} *(\mathbf{Q}, \mathbf{P}) \mathrm{d} \Gamma(\mathbf{P})+ \\
& \left.\sum_{\mathrm{i}=1}^{N_{\mathrm{c}}} \mathbf{R}_{\mathrm{ci}}(Q, P) \mathbf{u}_{\mathrm{ci}}^{*}(P)+\int_{\Omega \mathrm{g}} \mathrm{g}(\mathrm{p}) \underset{\sim_{\mathrm{g}}^{\mathrm{u}}}{*} \mathrm{Q}, \mathbf{p}\right) \mathrm{d} \Omega_{\mathrm{g}}(\mathrm{p})
\end{aligned}
$$

para a qual:

$$
\begin{aligned}
& \underset{\sim}{C}(Q)=\left[\begin{array}{cc}
\beta / 2 \pi & 0 \\
0 & 0
\end{array}\right] \\
& \underset{\sim}{\mathbf{u}}(\mathbf{Q})=\left\{\begin{array}{c}
\mathbf{w}(\mathbf{Q}) \\
\mathbf{w}\left(\mathbf{Q}^{\prime}\right)
\end{array}\right\}
\end{aligned}
$$

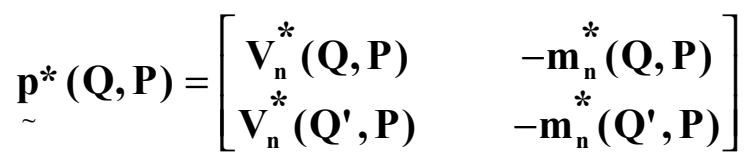

$$
\begin{aligned}
& \underset{\sim}{\mathbf{u}}(\mathbf{P})=\left\{\begin{array}{l}
\mathbf{u}_{1}(\mathbf{P}) \\
\mathbf{u}_{2}(\mathbf{P})
\end{array}\right\}=\left\{\begin{array}{c}
\mathbf{w}(\mathbf{P}) \\
\mathbf{w}, \mathbf{n}(\mathbf{P})
\end{array}\right\} \\
& \underset{\sim \mathrm{ci}}{\mathbf{p}}(\mathbf{Q}, \mathbf{P})=\left\{\begin{array}{c}
\mathbf{R}_{\mathrm{ci}}^{*}(\mathbf{Q}, \mathbf{P}) \\
\mathbf{R}_{\mathrm{ci}}^{*}\left(Q^{\prime}, P\right)
\end{array}\right\} \\
& \underset{\sim}{\mathbf{u}^{*}}(\mathbf{Q}, \mathbf{P})=\left[\begin{array}{lr}
\mathbf{w} *(\mathbf{Q}, \mathbf{P}) & -\mathbf{w},{ }_{\mathrm{n}}^{*}(\mathbf{Q}, \mathbf{P}) \\
\mathbf{w} *\left(\mathbf{Q}^{\prime}, \mathbf{P}\right) & -\mathbf{w},{ }_{\mathrm{n}}^{*}\left(\mathbf{Q}^{\prime}, \mathbf{P}\right)
\end{array}\right]
\end{aligned}
$$




$$
\begin{aligned}
& \underset{\sim}{\mathbf{p}}(\mathbf{P})=\left\{\begin{array}{l}
\mathbf{p}_{1}(\mathbf{P}) \\
\mathbf{p}_{2}(\mathbf{P})
\end{array}\right\}=\left\{\begin{array}{c}
\mathbf{V}_{\mathrm{n}}(\mathbf{P}) \\
\mathbf{m}_{\mathrm{n}}(\mathbf{P})
\end{array}\right\} \\
& \underset{\sim \mathrm{ci}}{\mathbf{u}}(\mathbf{Q}, \mathbf{P})=\left\{\begin{array}{c}
\mathbf{w}_{\mathrm{ci}}^{*}(\mathbf{Q}, \mathbf{P}) \\
\mathbf{w}_{\mathrm{ci}}^{*}\left(\mathbf{Q}^{\prime}, \mathbf{P}\right)
\end{array}\right\} \\
& \underset{\sim \mathrm{g}}{\mathbf{u}}(\mathbf{Q}, \mathbf{p})=\left\{\begin{array}{c}
\mathbf{w}^{*}\left(\mathbf{Q}^{\prime} \mathbf{p}\right) \\
\mathbf{w}^{*}\left(\mathbf{Q}^{\prime}, \mathbf{p}\right)
\end{array}\right\}
\end{aligned}
$$

Pode-se representar o sistema de equações na forma:

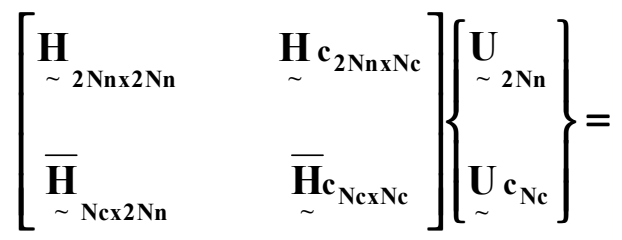

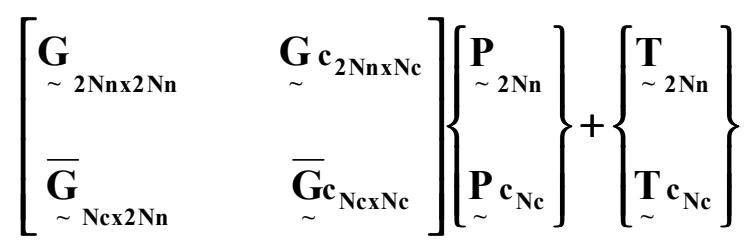

\subsection{5 - INTEGRAÇÃO SOBRE OS ELEMENTOS}

Na eq.(2.64), pode-se chamar de $\underset{\sim \mathbf{j}}{\mathbf{h}}(\mathbf{Q})$ e $\underset{\sim \mathbf{j}}{\mathbf{g}}(\mathbf{Q})$ as integrais:

$$
\underset{\sim j}{\mathbf{h}}(\mathbf{Q})=\int_{\Gamma_{j}}\left[\mathbf{p}_{\sim}^{*}(\mathbf{Q}, \mathbf{P}) \cdot \underset{\sim}{\Phi^{\mathrm{T}}}(\mathbf{P})\right] \mathrm{d} \Gamma_{\mathrm{j}}(\mathbf{P})
$$

e

$$
\left.\underset{\sim \mathbf{j}}{\mathbf{g}}(\mathbf{Q})=\int_{\Gamma_{\mathrm{j}}}{\underset{\sim}{\mathbf{u}}}^{*}(Q, P) \cdot \underset{\sim}{\Phi^{T}}(P)\right] \mathrm{d} \Gamma_{\mathbf{j}}(P)
$$

onde já foi visto que 


$$
\begin{aligned}
& \underset{\sim}{\mathbf{p}^{*}(Q, P)}=\left[\begin{array}{cc}
\mathbf{V}_{\mathbf{n}}^{*}(\mathbf{Q}, \mathbf{P}) & -\mathbf{m}_{\mathbf{n}}^{*}(\mathbf{Q}, \mathbf{P}) \\
\mathbf{V}_{\mathbf{n}}^{*}\left(\mathbf{Q}^{\prime}, \mathbf{P}\right) & -\mathbf{m}_{\mathbf{n}}^{*}\left(\mathbf{Q}^{\prime}, \mathbf{P}\right)
\end{array}\right] \\
& \underset{\sim}{\mathbf{u}^{*}}(\mathbf{Q}, \mathbf{P})=\left[\begin{array}{cc}
\mathbf{w}^{*}(\mathbf{Q}, \mathbf{P}) & -\mathbf{w}_{\mathbf{n}}^{*}(\mathbf{Q}, \mathbf{P}) \\
\mathbf{w}^{*}\left(\mathbf{Q}^{\prime}, \mathbf{P}\right) & -\mathbf{w},{ }_{\mathrm{n}}^{*}\left(\mathbf{Q}^{\prime}, \mathbf{P}\right)
\end{array}\right]
\end{aligned}
$$

e as funções interpoladoras das variáveis sobre os elementos

$$
\begin{aligned}
& \underset{\sim}{\Phi^{T}}=\left[\begin{array}{cccccc}
\phi_{1}(\mathbf{P}) & 0 & \phi_{2}(\mathbf{P}) & 0 & \phi_{3}(\mathbf{P}) & 0 \\
0 & \phi_{1}(\mathbf{P}) & 0 & \phi_{2}(\mathbf{P}) & 0 & \phi_{3}(\mathbf{P})
\end{array}\right] \\
& \Phi_{1}(\mathbf{P})=\frac{\left(\xi_{3}-\xi\right) \xi}{\xi_{1}\left(\xi_{3}-\xi_{1}\right)} \\
& \Phi_{2}(\mathbf{P})=1-\frac{\left(\xi_{3}+\xi_{1}\right) \xi}{\xi_{1} \xi_{3}}+\frac{\xi^{2}}{\xi_{1} \xi_{3}} \\
& \Phi_{3}(\mathbf{P})=\frac{\left(\xi_{1}-\xi_{)}\right)}{\xi_{3}\left(\xi_{1}-\xi_{3}\right)}
\end{aligned}
$$

As integrais da eq.(2.66) será facilmente efetuada se for expressa em termos de coordenadas adimensionais $\xi$ que é tal que:

$$
\Gamma_{\mathrm{j}}=\xi 1 / 2 \quad\left(\mathrm{~d} \Gamma_{\mathrm{j}} / \mathrm{d} \xi=1 / 2\right)
$$

Pode-se reescrever

$$
\begin{aligned}
& \underset{\sim \mathrm{j}}{\mathbf{h}}(\mathbf{Q})=\mathbf{h}_{\mathrm{ij}}^{\mathrm{n}}(\mathrm{Q})=\frac{1}{2} \int_{-1}^{1} \mathbf{p}_{\sim \xi}^{*}(Q, P) \Phi^{\mathrm{T}}(P) \mathrm{d} \xi(P) \\
& \underset{\sim \mathrm{j}}{\mathbf{g}}(\mathbf{Q})=\mathrm{g}_{\mathrm{ij}}^{\mathrm{n}}(\mathrm{Q})=\frac{1}{2} \int_{-1}^{1} \underset{\sim \xi}{\mathbf{u}^{*}}(Q, P) \Phi^{\mathrm{T}}(P) \mathrm{d} \xi(P)
\end{aligned}
$$

onde $\underset{\sim \xi}{\mathbf{p}}{ }_{\sim \boldsymbol{\sim}}^{*}$ e $\mathbf{u}_{\boldsymbol{\xi}}^{*}$ são $\underset{\sim \xi}{\mathbf{p}^{*}}$ e $\underset{\sim \xi}{\mathbf{u}^{*}}$ expressas em termos de $\xi$ e 1 o comprimento do elemento j. 


\subsection{1 - INTEGRAIS NUMÉRICAS:}

Efetuam-se as integrais na eq.(2.66) pela fórmula de quadratura de Gauss:

$$
\begin{aligned}
& \mathbf{h}_{\mathrm{ij}}^{\mathrm{n}}(\mathbf{Q})=\frac{1}{2} \sum_{\mathrm{i}=1}^{\mathrm{NG}} \varpi_{\mathrm{i}} \cdot{\underset{\sim}{\mathbf{p}_{\mathrm{i}}}}^{*}(\mathbf{Q}, \mathbf{P}) \cdot{\underset{\sim}{\Phi}}^{\mathrm{T}}(\mathbf{P}) \\
& \mathbf{g}_{\mathrm{ij}}^{\mathrm{n}}(\mathbf{Q})=\frac{1}{2} \sum_{\mathrm{i}=1}^{\mathrm{NG}} \bar{\Phi}_{\mathrm{i}} \cdot \underset{\sim \xi_{\mathrm{i}}}{\mathbf{u}^{*}}(\mathbf{Q}, \mathbf{P}) \cdot{\underset{\sim}{\Phi}}^{\mathrm{T}}(\mathbf{P})
\end{aligned}
$$

\subsection{2 - INTEGRAIS ANALÍTICAS:}

A integração efetuada analiticamente, é facilitada se as variáveis são escritas em função do raio $\mathbf{r}$, pois o sinal de $\Gamma_{\mathbf{j}}$ muda de acordo com a posição do nó singular. Estes resultados se encontram perfeitamente deduzido na vasta bibliografia sobre o assunto (ver, p.e., PAIVA(1987), CHUEIRI(1994) dentre outros), donde são aqui transferidos:

$$
\begin{aligned}
& h_{i 1}^{n}(Q)=\left(\int_{\Gamma_{j}} V * \Phi_{n} d \Gamma_{j}\right)_{i}=0 \\
& h_{i 2}^{n}(Q)=\lim _{\varepsilon \rightarrow 0}\left(\int_{a}^{\varepsilon} m_{n}^{*} \Phi_{n} d \Gamma_{j}+\int_{\varepsilon}^{b} m_{n}^{*} \Phi_{n} d \Gamma_{j}\right)_{i}= \\
& \frac{\ell_{j}}{4 \pi}\left\{C_{1}^{n}(1+v)\left[\phi_{2 s} \ln \left(\ell_{j} \phi_{2 s}\right)+\phi_{1 s} \ln \left(\ell_{j} \phi_{1 s}\right)-1\right]+\right. \\
& C_{2}^{n}(1+v)\left[\left(\phi_{2 s}\right)^{2} \ln \left(\ell_{j} \phi_{2 s}\right)-\left(\phi_{1 s}\right)^{2} \ln \left(\ell_{j} \phi_{1 s}\right)-\frac{\xi}{2}\right]+ \\
& C_{3}^{n}(1+v)\left[\frac{4}{3}\left(\phi_{2 s}\right)^{3} \ln \left(\ell_{j} \phi_{2 s}\right)+\frac{4}{3}\left(\phi_{1 s}\right)^{3} \ln \left(\ell_{j} \phi_{1 s}\right)-\frac{1}{9}\left(1+3 \xi^{2}\right)\right]+ \\
& \left.v\left[C_{1}^{n}+C_{2}^{n} \xi_{3}+\frac{C_{3}^{n}}{3}\left(1+3 \xi^{2}\right)\right]\right\}
\end{aligned}
$$




$$
\begin{aligned}
& \mathrm{g}_{\mathrm{i} 1}^{\mathrm{n}}(Q)=\lim _{\varepsilon \rightarrow 0}\left(\int_{\mathrm{a}}^{\varepsilon} \mathrm{w} * \Phi_{\mathrm{n}} \mathrm{d} \Gamma_{\mathrm{j}}+\int_{\varepsilon}^{\mathrm{b}} \mathrm{w} * \Phi_{\mathrm{n}} \mathrm{d} \Gamma_{\mathrm{j}}\right)= \\
& \frac{\ell_{\mathrm{j}}^{3}}{8 \pi \mathrm{D}}\left\{\frac{\mathrm{C}_{1}^{\mathrm{n}}}{3}\left[\left(\phi_{2 \mathrm{~s}}\right)^{3}\left(\ln \left(\ell_{\mathrm{j}} \phi_{2 \mathrm{~s}}\right)-\frac{5}{6}\right)-\left(\phi_{1 \mathrm{~s}}\right)^{3}\left(\ln \left(\ell_{\mathrm{j}} \phi_{1 \mathrm{~s}}\right)-\frac{5}{6}\right)\right]+\right. \\
& \frac{\mathrm{C}_{2}^{\mathrm{n}}}{2}\left[\left(\phi_{2 \mathrm{~s}}\right)^{4}\left(\ln \left(\ell_{\mathrm{j}} \phi_{2 \mathrm{~s}}\right)-\frac{3}{4}\right)-\left(\phi_{1 \mathrm{~s}}\right)^{4}\left(\ln \left(\ell_{\mathrm{j}} \phi_{1 \mathrm{~s}}\right)-\frac{3}{4}\right)\right]+ \\
& \left.\frac{4 C_{3}^{\mathrm{n}}}{5}\left[\left(\phi_{2 \mathrm{~s}}\right)^{5}\left(\ln \left(\ell_{\mathrm{j}} \phi_{2 \mathrm{~s}}\right)-\frac{7}{10}\right)+\left(\phi_{1 \mathrm{~s}}\right)^{5}\left(\ln \left(\ell_{\mathrm{j}} \phi_{1 \mathrm{~s}}\right)-\frac{7}{10}\right)\right]\right\} \\
& \mathrm{g}_{\mathrm{i} 2}^{\mathrm{n}}(Q)=\left(\int_{\Gamma_{\mathrm{j}}} \mathrm{w}_{\mathrm{n}}^{*} \Phi_{\mathrm{n}} \mathrm{d} \Gamma_{\mathrm{j}}\right)=0
\end{aligned}
$$

onde,

$$
\begin{aligned}
& C_{1}^{n}=\frac{-\xi^{2}+\left(\xi_{i}+\xi_{j}\right) \xi_{i}-\xi_{i} \xi_{j}}{\left(\xi_{i}-\xi_{n}\right)\left(\xi_{n}-\xi_{j}\right)} \\
& C_{2}^{n}=\frac{2 \xi_{i}-\left(\xi_{i}+\xi_{j}\right)}{\left(\xi_{i}-\xi_{n}\right)\left(\xi_{n}-\xi_{j}\right)} \\
& C_{1}^{n}=\frac{-1}{\left(\xi_{i}-\xi_{n}\right)\left(\xi_{n}-\xi_{j}\right)}
\end{aligned}
$$

$\operatorname{para} \mathbf{n}, \mathbf{i}, \mathbf{j}=\mathbf{1}, \mathbf{2}, \mathbf{3}$ e $\mathbf{n} \neq \mathbf{i} \neq \mathbf{j}$.

\subsection{3 - INTEGRAIS NUMÉRICAS SUBELEMENTADAS}

A proximidade do ponto de carga em relação aos elementos sobre os quais será feita a integração, aumenta a influência no valor da variável a ser calculada. Melhorase este resultado se a distância entre o ponto de carga e o ponto médio do elemento não for muito grande. Uma técnica eficiente, usada por alguns pesquisadores (CHAVES (1997), FERNANDES(1998), ROCHA(1999)), consiste em subdividir o 
elemento, dependendo da posição do ponto de colocação em relação a ele. Neste novo subdomínio realiza-se a integração numérica.

A condição da subdivisão pode ser baseada na limitação da distância entre estes pontos a no mínimo o comprimento do elemento que, quanto menor, maior será o número de Gauss para efeito de integração numérica. 


\section{CAPÍTULO 3 - MÉTODO DOS ELEMENTOS DE CONTORNO APLICADO À ANÁLISE DE CHAPAS}

\section{1 - INTRODUÇÃO}

Com o objetivo de desenvolver a formulação para análise linear de chapas através do MEC, serão expostos neste capítulo os aspectos necessários para a elaboração de um algoritmo. Este algoritmo será utilizado, portanto, como uma rotina básica para o cálculo de estruturas sob estado plano de tensão. Algumas simplificações adotadas para o presente trabalho serão também discutidas.

O que aqui se apresentará sobre a teoria de chapas planas pelo MEC é uma breve recapitulação do que já se encontra bem estudado na bibliografia sobre o assunto. (p.e. VENTURINI(1988), BREBBIA \& DOMINGUES(1989), PALERMO(1989)). A análise de chapas, ou placas planas sob estado plano de tensão ("plate stretching” - BREBBIA \& DOMINGUES(1989)), é também uma simplificação do problema tridimensional na Teoria da Elasticidade. Serão revistas as 
equações básicas desta teoria, apontando para o fato de que, normalmente no MEC, a formulação é desenvolvida com base no estado plano de deformações (EPD) e posteriormente convertidas ao estado plano de tensões (EPT). Conforme já se sabe, esta conversão é feita através de uma simples substituição de constantes elásticas por valores equivalentes, como se verá adiante (ver item 3.5).

\section{2 - HIPÓTESES BÁSICAS}

Com a intenção de facilitar o entendimento das condições básicas de cálculo no estado plano de tensões (EPT), considere-se o elemento infinitesimal da fig. 3.1, onde a origem do sistema de referências $\mathbf{x}_{1} \mathbf{x}_{2} \mathbf{x}_{3}$ pertence ao plano médio da chapa.

Para o EPT, portanto, assume-se que:

-o material de que é composta a chapa é suposto homogêneo, isotrópico e de comportamento elástico linear;

-a espessura da chapa t é pequena se comparada às suas outras dimensões;

-as forças de volume ( $\mathbf{b}_{\mathbf{i}}$; ver fig. 3.2) atuam apenas no plano $\mathbf{x}_{1}-\mathbf{x}_{2}\left(\mathbf{b}_{3}=\mathbf{0}\right)$ e não dependem $\mathbf{x}_{3}\left(\mathbf{b}_{1}=\mathbf{b}_{1}\left(\mathbf{x}_{1}, \mathbf{x}_{2}\right)\right.$ e $\left.\mathbf{b}_{2}=\mathbf{b}_{2}\left(\mathbf{x}_{1}, \mathbf{x}_{2}\right)\right)$;

-também as forças de superfície ( $\mathbf{p}_{\mathrm{i}}$; ver fig. 3.3) atuam no plano $\mathbf{x}_{1}-\mathbf{x}_{2}$ apenas $\left(\mathbf{p}_{3}=\mathbf{0}\right)$ e independem $\mathbf{x}_{3}\left(\mathbf{p}_{1}=\mathbf{p}_{1}\left(\mathbf{x}_{\mathbf{1}}, \mathbf{x}_{2}\right)\right.$ e $\left.\mathbf{p}_{2}=\mathbf{p}_{2}\left(\mathbf{x}_{\mathbf{1}}, \mathbf{x}_{2}\right)\right)$;

-não há forças de superfície atuantes nas superfícies externas da chapa, isto é, em $\mathbf{x}_{3}= \pm \mathbf{t} / \mathbf{2}, \mathbf{p}_{\mathrm{j}}=0$ (ver fig.(3.3));

-como conseqüência das hipóteses acima, $\boldsymbol{\sigma}_{3 \mathrm{i}} \cong 0,(\mathrm{i}=1,3)$ e $\boldsymbol{\sigma}_{11}, \boldsymbol{\sigma}_{22}, \boldsymbol{\sigma}_{12}$, bem como $\mathbf{u}_{11}, \mathbf{u}_{22}, \mathbf{u}_{12}$, variam apenas em função de $\mathbf{x}_{1}$ e $\mathbf{x}_{2}$.

Com base nestas condições, elaboram-se as equações básicas para a definição deste fenômeno, em que se consideram os estados planos de tensão e deformação, relacionados, como se sabe, por equações constitutivas.

Antes, porém, é importante lembrar que, como mencionado na introdução deste capítulo, o procedimento para a obtenção da descrição do problema de chapas através 
do MEC, é inicialmente desenvolvido com base no EPD e, quando necessário, convertidas ao EPT através do uso de constantes do material. Por isso, listam-se abaixo de forma resumida as hipóteses básicas de cálculo de ambos estados (ver figs. 3.1, a 3.3):

a) Para o EPT (i=1,3 e k,j=1,2):

$$
\begin{aligned}
& \sigma_{3 \mathrm{i}}=\mathbf{0} \\
& \sigma_{\mathrm{kj}}=\sigma_{\mathrm{kj}}\left(\mathbf{x}_{\mathbf{1}}, \mathbf{x}_{2}\right) \\
& \mathbf{b}_{3}=\mathbf{0} \\
& \mathbf{b}_{\mathbf{j}}=\mathbf{b}_{\mathbf{j}}\left(\mathbf{x}_{\mathbf{1}}, \mathbf{x}_{\mathbf{2}}\right) \\
& \mathbf{p}_{\mathbf{3}}=\mathbf{0} \\
& \mathbf{p}_{\mathbf{j}}=\mathbf{p}_{\mathbf{j}}\left(\mathbf{x}_{1}, \mathbf{x}_{\mathbf{2}}\right) \\
& \mathrm{em} \mathbf{x}_{3}= \pm \mathbf{t} / \mathbf{2}, \mathbf{p}_{\mathrm{i}}=0 \\
& \mathbf{u}_{\mathbf{j}}=\mathbf{u}_{\mathbf{j}}\left(\mathbf{x}_{\mathbf{1}}, \mathbf{x}_{\mathbf{2}}\right) \\
& \mathbf{u}_{3} \neq \mathbf{0}
\end{aligned}
$$

b) Para o EPD (i=1,3 e k,j=1,2):

$$
\begin{aligned}
& \varepsilon_{3 \mathrm{i}}=\mathbf{0} \\
& \varepsilon_{\mathrm{kj}}=\varepsilon_{\mathrm{kj}}\left(\mathbf{x}_{1}, \mathbf{x}_{2}\right) \\
& \mathbf{u}_{3}=\mathbf{0} \\
& \mathbf{u}_{\mathrm{j}}=\mathbf{u}_{\mathbf{j}}\left(\mathbf{x}_{1}, \mathbf{x}_{2}\right) \\
& \operatorname{em~} \mathbf{x}_{3}= \pm \mathbf{t} / 2, \mathbf{u}_{\mathrm{i}}=0 \\
& \boldsymbol{\sigma}_{33}
\end{aligned}
$$

e sobre a superfície cilíndrica do corpo alongado, perpendicular ao plano vertical:

$$
\begin{aligned}
& \mathbf{b}_{3}=\mathbf{0} \\
& \mathbf{b}_{\mathbf{j}}=\mathbf{b}_{\mathbf{j}}\left(\mathbf{x}_{\mathbf{1}}, \mathbf{x}_{2}\right) \\
& \mathbf{p}_{3}=\mathbf{0} \\
& \mathbf{p}_{\mathbf{j}}=\mathbf{p}_{\mathbf{j}}\left(\mathbf{x}_{1}, \mathbf{x}_{2}\right)
\end{aligned}
$$




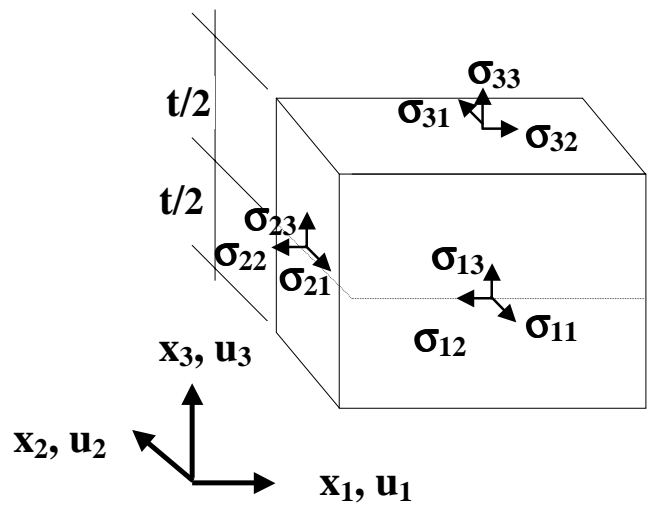

Fig. 3.1 - Estado de Tensões sobre elemento infinitesimal

\section{3 - ESTADO DE TENSÕES $\left(\sigma_{\mathrm{ij}}\right)$}

Considere-se o elemento infinitesimal da fig.3.1. Nela apresentam-se as componentes de tensão atuantes no elemento que se relacionam entre si através de equações de equilíbrio (a origem do sistema de coordenadas pertence ao plano médio da chapa). Das equações de equilíbrio de momento, obtém-se, para $\mathbf{i , j}=\mathbf{1 , 3}$ :

$$
\sigma_{\mathrm{ij}}=\sigma_{\mathrm{ji}} \quad \mathbf{i} \neq \mathbf{j}
$$

O equilíbrio de forças fornece, definindo-se $\mathbf{b}_{\mathbf{i}}$ como forças de volume (fig.3.2):

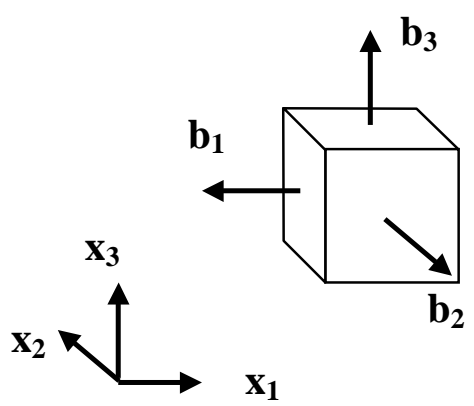

Fig. 3.2 - Forças de volume 


$$
\sigma_{\mathrm{ij}, \mathrm{j}}+\mathbf{b}_{\mathrm{i}}=\mathbf{0} \quad \mathbf{j}, \mathbf{i}=\mathbf{1 , 3}
$$

As forças de superfície $\mathbf{p}_{\mathbf{i}}$ ("tractions” - BREBBIA \& DOMINGUES(1989)), são resultantes da projeção das componentes de tensão sobre um diferencial do contorno da chapa $(\mathbf{d} \Gamma)$. Portanto, sendo $\mathbf{n}_{\mathbf{j}}=\cos \left(\mathbf{n}, \mathbf{x}_{\mathbf{j}}\right)$ os cossenos diretores do vetor normalà superfície $\mathbf{n}$, calculam-se as $\mathbf{p}_{\mathbf{i}}$ as na forma (fig.3.3):

$$
\mathbf{p}_{\mathrm{i}}=\boldsymbol{\sigma}_{\mathrm{ij}} \cdot \mathbf{n}_{\mathrm{j}}
$$

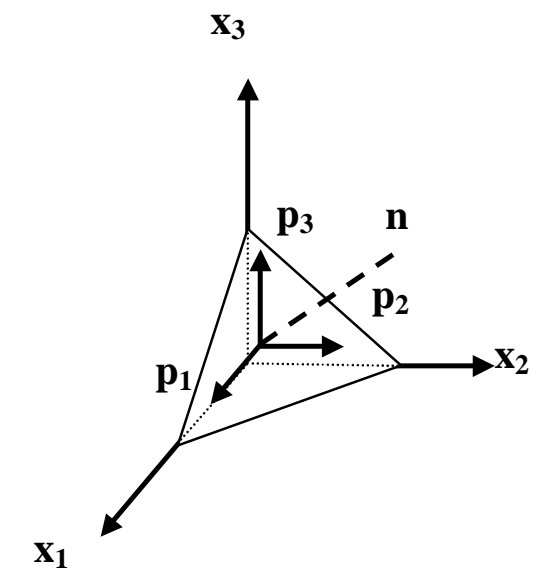

Fig. 3.3 - Forças de superfície

\section{4 - ESTADO DE DEFORMAÇÕES $\left(\varepsilon_{\mathrm{ij}}\right)$}

As componentes de deformação $\left(\boldsymbol{\varepsilon}_{\mathrm{ij}}\right)$ correspondentes às de tensão expostas no item anterior, são função dos deslocamentos $\mathbf{u}_{\mathbf{i}}$ (fig.3.1) de forma que, novamente:

$$
\varepsilon_{\mathrm{ij}}=\frac{1}{2}\left(\mathrm{u}_{\mathrm{i}, \mathrm{j}}+\mathrm{u}_{\mathrm{j}, \mathrm{i}}\right) \quad \mathrm{i}, \mathrm{j}=1,3
$$

onde

$$
\varepsilon_{\mathrm{ij}}=\varepsilon_{\mathrm{ji}} \quad \mathrm{i} \neq \mathbf{j}
$$




\section{5 - RELAÇÕES CONSTITUTIVAS}

Como se sabe, os estados de tensão e deformação estão relacionados por equações constitutivas do material em estudo (Hooke). Sendo ele de propriedades lineares, pode-se simplificadamente escrever esta relação, utilizando-se as constantes de Lamé, na seguinte forma indicial:

$$
\sigma_{\mathrm{ij}}=2 \mu \varepsilon_{\mathrm{ij}}+\lambda \delta_{\mathrm{ij}} \varepsilon_{\mathrm{kk}} \quad \mathrm{i}, \mathrm{j}, \mathrm{k}=1,3
$$

sendo

$$
\boldsymbol{\mu}=\mathbf{G}
$$

$\mathrm{e}$

$$
\lambda=\frac{2 G v}{(1-2 v)}
$$

lembrando que (eq.2.5)

$$
\mathbf{G}=\frac{\mathbf{E}}{2(1+v)}
$$

Conforme já comentado, estados planos são simplificações do problema elastostático tridimensional. Então, a eq.3.6 para o EPD, de acordo com as condições básicas listada no item 3.2, não muda em sua forma, mas a variação de $\mathbf{i}, \mathbf{j}, \mathbf{k}$ agora se dá entre 1 e 2. Para o EDT também i,j,k=1,2 , porém, a eq.3.6 reescreve-se:

$$
\sigma_{\mathrm{ij}}=2 \mu \varepsilon_{\mathrm{ij}}+\frac{2 \mu \lambda}{2 \mu+\lambda} \delta_{\mathrm{ij}} \varepsilon_{\mathrm{kk}} \quad \mathrm{i}, \mathrm{j}, \mathrm{k}=1,2
$$

BREBBIA \& DOMINGUES(1989) comentam que conhece-se a solução fundamental para o problema do EPD, pois isto, usa-se elaborar a formulação para este caso e depois migrar para o EPT, através da relação: 


$$
v^{\prime}=\frac{v}{(1+v)}
$$

que é proveniente da devida manipulação entre as eqs.3.6 e 3.8, e deve ser incorporado no cálculo das constantes da eqs. 2.5 e 3.7 .

\section{6 - EQUAÇÃO DIFERENCIAL PARA O EPT (Navier)}

Para se obter a forma diferencial para a equação de equilíbrio (eq. 3.2) do elemento da fig. 3.1, pode-se substituir os valores das eqs. 3.4 e 3.6, obtendo-se:

$$
\frac{1}{1-2 v} u_{j, j i}+u_{i, j j}+\frac{1}{\mu} b_{i}=0
$$

que é a equação equilíbrio em termos de deslocamentos (equação de Navier), cuja solução deve satisfazer a eq. 3.2, agora expressa em termos de deslocamentos, novamente a partir da substituição das eqs. 3.4 e 3.6 pelas variáveis que envolve:

$$
\mathbf{p}_{\mathrm{i}}=\mu\left(\mathbf{u}_{\mathrm{i}, \mathrm{j}}+\mathbf{u}_{\mathrm{j}, \mathrm{i}}\right) \mathbf{n}_{\mathrm{j}}+\lambda \mathbf{u}_{\mathrm{k}, \mathrm{k}} \mathbf{n}_{\mathrm{i}}
$$

\section{7 - SOLUÇÃO FUNDAMENTAL (*)}

Conforme visto no item 2.10, define-se como solução fundamental a solução do problema elástico a um domínio infinito de mesmo material, submetido a uma força unitária aplicada num determinado ponto. Para o EPT, obtém-se esta solução a partir da equação de equilíbrio (eq. 3.2 ou de Navier, eq. 3.10) escrita na forma:

$$
\sigma_{\mathrm{ij}, \mathrm{j}}(\mathbf{q}, \mathbf{p})+\Delta_{\mathrm{i}}(\mathbf{q}, \mathbf{p})=\mathbf{0}
$$


sendo a distribuição do delta de Dirac $\Delta(\mathbf{q}, \mathbf{p})$, conforme já visto, a representação matemática da carga unitária aplicada num ponto q, na direção i. As propriedades desta distribuição, vista no item $\mathbf{2 . 1 0}$, são aqui transcritas:

$$
\begin{aligned}
& \Delta(\mathrm{q}, \mathrm{p})=0 \quad \text { para } \quad \mathrm{p} \neq \mathrm{q} \\
& \Delta(\mathrm{q}, \mathrm{p})=\infty \quad \text { para } \quad \mathrm{p}=\mathrm{q} \\
& \int_{\Omega} \Delta(\mathrm{q}, \mathrm{p}) \mathrm{d} \Omega=\mathbf{1}
\end{aligned}
$$

$\mathrm{e}$

$$
\int_{\Omega} \phi(p) \Delta(q, p) d \Omega=\phi(q)
$$

$\operatorname{com} \phi(p)$ representando uma função contínua.

Porém, por não constituir uma equação de Laplace, a eq. 3.10 deve ser manipulada para obter-se a solução fundamental, neste caso. BREBBIA \& DOMINGUES(1989), PALERMO(1989) dentre outros, utilizaram a representação do deslocamento em termos do vetor de $\operatorname{Galerkin}\left(\mathbf{F}_{\mathbf{i}}\right)$, de forma que:

$$
\mathbf{u}_{\mathrm{j}}=\mathbf{F}_{\mathrm{j}, \mathrm{mm}}-\frac{1}{2(1-v)} \mathbf{F}_{\mathrm{m}, \mathrm{jm}}
$$

que substituída, juntamente com a função delta de Dirac na eq. 3.10, tem-se por solução

$$
u_{\mathrm{ik}}^{*}(q, p)=\frac{1}{8 \pi \mu(1-v)}\left[(3-4 v) \ln \left(\frac{1}{r}\right) \delta_{\mathrm{ik}}+r_{,_{\mathrm{i}}} \mathbf{r}_{\mathrm{k}}\right]
$$

que é a solução fundamental para o problema bidimensional do estado plano de deformação (EPD), sendo, $\mathbf{r}$ a distância entre o ponto de resposta (p) e ponto de carga (q), conforme visto no item 2.10. Para transformar esta solução para o EPT, basta substituir $\mathbf{v}$ por $\mathbf{v}$, conforme eq. 3.9. 
A partir da solução $\mathbf{u}^{*}{ }_{\text {ik }}(\mathbf{q}, \mathbf{p})$, pode-se obter, através das eq. 3.4, 3.6 e 3.3, as soluções para $\boldsymbol{\varepsilon}^{*}{ }_{\mathrm{ik}}(\mathbf{q}, \mathbf{p})$ e, conseqüentemente, $\operatorname{para} \boldsymbol{\sigma}^{*}{ }_{\mathrm{ik}}(\mathbf{q}, \mathbf{p})$ e:

$$
\mathbf{p}_{\mathrm{ik}}^{*}(\mathbf{q}, \mathbf{p})=-\frac{1}{4 \pi(1-v) r}\left[\mathbf{r},{ }_{\mathrm{n}}\left\{(1-2 v) \delta+2 \mathbf{r},{ }_{\mathrm{i}} \mathbf{r}_{\mathrm{k}}\right\}-(1-2 v)\left(\mathbf{r},{ }_{\mathrm{i}} \mathbf{n}_{\mathrm{k}}-\mathbf{r},{ }_{\mathrm{k}} \mathbf{n}_{\mathrm{i}}\right)\right]
$$

\section{8 - REPRESENTAÇÃO INTEGRAL PARA PONTOS DO DOMÍNIO}

Deseja-se obter a formulação integral que define o problema de chapas a partir do teorema da reciprocidade (ver, p.e., VENTURINI(1988)). Considere-se o domínio finito $\boldsymbol{\Omega}$ da chapa em questão, contido num domínio infinito $\boldsymbol{\Omega}^{*}$, e aos quais associam-se o carregamento real e outro correspondente ao problema fundamental, respectivamente. São relativos ao problema real os deslocamentos $\mathbf{u}_{\mathrm{k}}(\mathbf{p})$, as tensões $\boldsymbol{\sigma}_{\mathrm{ij}}(\mathbf{p})$, as deformações $\boldsymbol{\varepsilon}_{\mathrm{ij}}(\mathbf{p})$ e as forças volumétricas $\mathbf{b}_{\mathrm{i}}(\mathbf{p})$. A ele também pertencem os valores prescritos $\overline{\mathbf{u}}_{\mathbf{i}}(\mathbf{P})$, sobre $\Gamma_{1}$ e $\overline{\mathbf{p}}_{\mathbf{i}}(\mathbf{P})$, em $\Gamma_{2}$. Ao problema fundamental referem-se $\mathbf{u}_{\mathrm{k}}^{*}(\mathbf{q}, \mathbf{p}), \boldsymbol{\sigma}_{\mathrm{ij}}^{*}(\mathbf{q}, \mathbf{p}), \boldsymbol{\varepsilon}_{\mathrm{ij}}^{*}(\mathbf{q}, \mathbf{p})$ e das forças de superfície $\mathbf{p}_{\mathrm{i}}^{*}(\mathbf{q}, \mathbf{p})$. Através do teorema da reciprocidade, relacionam-se os estados de tensão e deformação de ambos os problemas de forma que:

$$
\int_{\Omega} \sigma_{\mathrm{ij}} \varepsilon_{\mathrm{ij}}^{*} \mathrm{~d} \Omega=\int_{\Omega} \varepsilon_{\mathrm{ij}} \sigma_{\mathrm{ij}}^{*} \mathrm{~d} \Omega \quad \mathrm{i}, \mathrm{j}=1,2
$$

da qual obtém-se, integrando por partes uma vez, a seguinte equação:

$$
\int_{\Gamma_{2}} p_{i} u_{i}^{*} d \Gamma-\int_{\Omega} \sigma_{i j, j} u_{i}^{*} d \Omega=\int_{\Gamma_{1}} u_{i} p_{i}^{*} d \Gamma-\int_{\Omega} \sigma_{i j, j}^{*} u_{i} d \Omega \quad i, j=1,2(3.16)
$$

Substituindo-se as eq. 3.2 e 3.12 na eq. 3.18 e considerando-se as propriedades da função delta de Dirac (eqs. 3.12a-c), obtém-se, portanto, a formulação integral para cálculo dos deslocamentos de pontos (q) localizados no interior do domínio da chapa, a partir de valores de contorno: 


$$
u_{i}(q)=-\int_{\Gamma} p_{i}^{*}(q, P) u_{i}(P) d \Gamma(P)+\int_{\Gamma} u_{i}^{*}(q, P) p_{i}(P) d \Gamma(P)+\int_{\substack{\Omega \\ i, j=1,2}}^{*}(q, p) b_{i}(p) d \Omega(p)
$$

\section{9 - REPRESENTAÇÃO INTEGRAL PARA PONTOS DO CONTORNO}

Da mesma forma que no capítulo anterior, será utilizado o mesmo artifício para a elaboração da formulação para o problema do EPT pelo MEC, a partir da eq. 3.17, quando o ponto fonte q está locado no contorno. Aqui, usa-se novamente o domínio da placa acrescido de um setor de raio $\xi$ centrado em $\mathbf{q}$ (fig.(2.6)). Com os novos domínio $\left(\boldsymbol{\Omega}+\boldsymbol{\Omega}_{\xi}\right)$ e contorno $\left(\boldsymbol{\Gamma}-\bar{\Gamma}+\Gamma_{\xi}\right)$ definidos a eq.(3.17) é então modificada, de forma a calcular o deslocamento $\mathbf{w}(\mathbf{Q})$, sendo que $\mathbf{Q}$ é um ponto do contorno, ou seja, o raio $\xi$ tende a zero.

Procedendo-se de forma análoga à que gerou a equação integral para pontos de contorno no problema de flexão de placas, obtém-se a representação integral dos deslocamentos dos pontos de contorno de chapas:

$$
\begin{gathered}
c_{i}(Q) u_{i}(Q)=-\int_{\Gamma} p_{i}^{*}(Q, P) u_{i}(P) d \Gamma(P)+\int_{\Gamma} u_{i}^{*}(Q, P) p_{i}(P) d \Gamma(P)+\int_{\Omega} u_{i}^{*}(Q, p) b_{i}(p) d \Omega(p) \\
i, j=1,2
\end{gathered}
$$

onde os valores para $\mathbf{u}_{\mathbf{i}}{ }^{*}$ e $\mathbf{p}_{\mathbf{i}}{ }^{*}$ são dados nas eq. 3.13 e 3.14. $\mathbf{c}_{\mathbf{i}}(\mathbf{Q})$ contém as características das tangentes que determinam a angulosidade do contorno em $\mathbf{Q}$.

\subsection{0 - O MEC APLICADO À ANÁLISE DE CHAPAS SOB EPT}

Da mesma forma que visto no problema de flexão de placas (cap. 2), há que se dividir o contorno da chapa em elementos, de valores para as variáveis $\mathbf{u}_{\mathrm{i}}$ e $\mathbf{p}_{\mathrm{i}}$ sobre 
eles calculados por funções aproximadoras dos valores nodais destas variáveis. Novamente, a integral sobre todo o contorno da chapa é substituída pela soma das integrações sobre o contorno de cada elemento, transformando a equação integral numa equação algébrica envolvendo valores nodais daquelas variáveis no contorno. Um sistema de equações é construído ao se escrever equações para os nós do contorno (eq. 3.18), uma para cada direção ị $(\mathbf{i}=1,2)$ por nó, cujo vetor de incógnitas possui os valores nodais de deslocamentos e forças de superfície. Uma outra opção, que foi inclusive adotado neste trabalho, construir este sistema com a eq. 3.18 escrita para nós externos à placa, evitando, assim, a resolução de integrais singulares. A imposição das condições de contorno é suficiente para resolver este sistema, possibilitando o cálculo de outros valores em qualquer ponto do domínio da chapa, através da eq. 3.17.

Como foi feito para ao problema de flexão de placas, a rotina desenvolvida, com base na teoria estudada até então, se refere a elementos de contorno de geometria aproximada por funções lineares e a distribuição das variáveis sobre eles aproximadas por funções quadráticas.

Assumindo-se esta subdivisão do contorno $\Gamma$ em $\mathbf{N}_{\mathbf{e}}$ elementos, cada elemento $\mathbf{j}$ com contorno $\Gamma_{\mathbf{j}}$, pode-se reescrever a eq. $\mathbf{3 . 1 8}$ na forma:

$$
\begin{aligned}
& \underset{\sim}{\mathbf{c}}(\mathbf{Q}) \underset{\sim}{\mathbf{u}}(\mathbf{Q})+\sum_{\mathbf{j}=1}^{\mathbf{N}_{\mathrm{e}}} \int_{\Gamma_{\mathbf{j}}}\left(\mathbf{p}^{*}(\mathbf{Q}, \mathbf{P}) \cdot \underset{\sim}{\mathbf{u}}(\mathbf{P}) \mathbf{d} \Gamma_{\mathbf{j}}(\mathbf{P})=\sum_{\mathbf{j}=1}^{\mathbf{N}_{\mathrm{e}}} \int_{\Gamma_{\mathbf{j}}}\left(\underline{\sim}^{*}(\mathbf{Q}, \mathbf{P}) \cdot \underset{\sim}{\mathbf{p}}(\mathbf{P}) \mathbf{d} \Gamma_{\mathbf{j}}(\mathbf{P})\right.\right. \\
& +\int_{\Omega_{b}}^{\mathbf{u}} *(Q, p) \underset{\sim}{b}(p) d \Omega_{b}(p)
\end{aligned}
$$

onde

$$
\underset{\sim}{\mathbf{c}}(\mathbf{Q})=\left[\begin{array}{ll}
\mathbf{c}_{11} & \mathbf{c}_{12} \\
\mathbf{c}_{21} & \mathbf{c}_{22}
\end{array}\right]
$$




$$
\begin{aligned}
& \underset{\sim}{\mathbf{u}}(\mathbf{Q})=\left\{\begin{array}{l}
\mathbf{u}_{1}(\mathbf{Q}) \\
\mathbf{u}_{2}(\mathbf{Q})
\end{array}\right\} \\
& \mathbf{p}^{*}(\mathbf{Q}, \mathbf{P})=\left[\begin{array}{cc}
\mathbf{p}_{11}^{*}(\mathbf{Q}, \mathbf{P}) & \mathbf{p}_{12}^{*}(\mathbf{Q}, \mathbf{P}) \\
\mathbf{p}_{12}^{*}\left(\mathbf{Q}^{\prime}, \mathbf{P}\right) & \mathbf{p}_{22}^{*}\left(\mathbf{Q}^{\prime}, \mathbf{P}\right)
\end{array}\right] \\
& \underline{u}(\mathbf{P})=\left\{\begin{array}{l}
\mathbf{u}_{1}(\mathbf{P}) \\
\mathbf{u}_{2}(\mathbf{P})
\end{array}\right\} \\
& \underset{\sim}{\mathbf{u}^{*}}(\mathbf{Q}, \mathbf{P})=\left[\begin{array}{ll}
\mathbf{u}_{11}^{*}(\mathbf{Q}, \mathbf{P}) & \mathbf{u}_{12}^{*}(\mathbf{Q}, \mathbf{P}) \\
\mathbf{u}_{21}^{*}(\mathbf{Q}, \mathbf{P}) & \mathbf{u}_{22}^{*}(\mathbf{Q}, \mathbf{P})
\end{array}\right] \\
& \underline{\mathbf{p}}(\mathbf{P})=\left\{\begin{array}{l}
\mathbf{p}_{1}(\mathbf{P}) \\
\mathbf{p}_{2}(\mathbf{P})
\end{array}\right\} \\
& \underset{\sim \mathbf{b}}{\mathbf{u}^{*}}(\mathbf{Q}, \mathbf{p})=\left[\begin{array}{ll}
\mathbf{u}_{11}^{*}(\mathbf{Q}, \mathbf{p}) & \mathbf{u}_{12}^{*}(\mathbf{Q}, \mathbf{p}) \\
\mathbf{u}_{21}^{*}(\mathbf{Q}, \mathbf{p}) & \mathbf{u}_{22}^{*}(\mathbf{Q}, \mathbf{p})
\end{array}\right] \\
& \underset{\sim}{\mathbf{b}}(\mathbf{p})=\left\{\begin{array}{l}
\mathbf{b}_{1}(\mathbf{p}) \\
\mathbf{b}_{2}(\mathbf{p})
\end{array}\right\}
\end{aligned}
$$

Observe-se que, como resultado do artifício usado no item 3.9, o valor dos componentes da matriz $\mathbf{c}(\mathbf{Q})$ dependerá do contorno ao qual pertence o ponto $\mathbf{Q}$. Se $\mathbf{Q}$ pertence ao domínio interno da chapa, então, $\mathbf{c}(\mathbf{Q})=\mathbf{I}_{2}$ (identidade de segunda ordem). Para $\mathbf{Q}$ situado no contorno sem a presença de angulosidade ("smooth

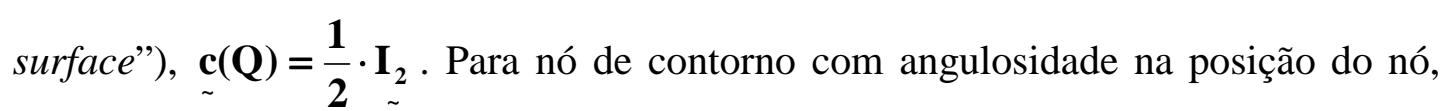
pode-se demonstrar que (BREBBIA \& DOMINGUES(1989)):

$$
\underset{\sim}{\mathbf{c}}(\mathbf{Q})=\underset{\sim}{\mathbf{I}_{2}}+\underset{\sim}{\overline{\mathbf{c}}}(\mathbf{Q})
$$


para a qual

$$
\bar{\sim}(Q)=\frac{-1}{8 \pi(1-v)}\left[\begin{array}{cc}
\overline{c_{11}}(Q) & \overline{c_{12}}(Q) \\
\tilde{\bar{c}}(Q) & \overline{\tilde{c}}(Q) \\
\tilde{c_{22}}(Q)
\end{array}\right]
$$

onde

$$
\begin{aligned}
& \bar{\sim} \overline{c_{11}}(Q)=4(1-v)\left(\pi+\theta_{2}-\theta_{1}\right)+\operatorname{sen} 2 \theta_{1}-\operatorname{sen} 2 \theta_{2} \\
& \overline{c_{11}}(Q)=4(1-v)\left(\pi+\theta_{2}-\theta_{1}\right)+\operatorname{sen} 2 \theta_{2}-\operatorname{sen} 2 \theta_{1} \\
& \overline{c_{12}}(Q)=\bar{\sim} \overline{c_{21}}(Q)=\cos 2 \theta_{2}-\cos 2 \theta_{1}
\end{aligned}
$$

Como no problema de flexão de placas, há a possibilidade de se usar o mesmo artifício alternativo que consiste em escrever a eq. (3.18) para um nó localizado fora do domínio da placa relacionado a um nó do contorno em estudo. Deve-se lembrar que, estes pontos externos são posicionados na direção normal ao contorno no ponto em questão, a uma distância $\underline{\mathbf{d}}$ do mesmo, (fig.2.8) que é tal que:

$$
\mathrm{d}=\alpha \ell_{\mathbf{m}}
$$

sendo $\ell_{\mathrm{m}} \mathrm{o}$ valor médio entre os comprimentos dos elementos concorrentes neste ponto do contorno ou o comprimento do elemento, caso o nó em questão seja o nó central do elemento.

Neste caso, $\underset{\sim}{\mathbf{c}}(\mathbf{Q})=\mathbf{0}_{2}$, além de ser evitado o cálculo das integrais analíticas a serem comentadas adiante. 
Definindo-se o vetor valores nodais de deslocamento e esforços de um elemento como $\tilde{\sim}^{\mathbf{N}}$ e $\underline{\sim}^{\mathbf{N}}$, respectivamente, de forma que:

$$
\begin{aligned}
& \underline{\sim}^{N}=\left\{\mathbf{U}_{i}^{N}\right\}=\left\{\begin{array}{c}
\mathbf{U}_{1}^{N} \\
\mathbf{U}_{2}^{N}
\end{array}\right\} \\
& \underline{\mathbf{P}}^{\mathrm{N}}=\left\{\mathbf{P}_{\mathrm{i}}^{\mathrm{N}}\right\}=\left\{\begin{array}{l}
\mathbf{P}_{1}^{\mathrm{N}} \\
\mathbf{P}_{2}^{\mathrm{N}}
\end{array}\right\}
\end{aligned}
$$

sendo que $\underline{\mathbf{N}}$ representa o número do ponto do elemento de contorno (1 a 3 para o elemento quadrático). Para um ponto genérico $\underline{\mathbf{P}}$ deste elemento:

$$
\begin{aligned}
& \underset{\sim}{\mathbf{u}}(\mathbf{P})=\left\{\begin{array}{l}
\mathbf{u}_{1}(\mathbf{P}) \\
\mathbf{u}_{2}(\mathbf{P})
\end{array}\right\}={\underset{\sim}{\Phi}}^{\mathrm{T}}(\mathbf{P}) \cdot \underline{\sim}^{\mathrm{N}} \\
& \underset{\sim}{\mathbf{p}(\mathbf{P})}=\left\{\begin{array}{l}
\mathbf{p}_{1}(\mathbf{P}) \\
\mathbf{p}_{2}(\mathbf{P})
\end{array}\right\}=\underline{\sim}^{\mathbf{T}}(\mathbf{P}) \cdot \underline{\mathbf{P}}^{\mathrm{N}}
\end{aligned}
$$

onde, conforme eq. 2.55

$$
{\underset{\sim}{\Phi}}^{\mathrm{T}}=\left[\begin{array}{cccccc}
\phi_{1}(\mathbf{P}) & \mathbf{0} & \phi_{2}(\mathbf{P}) & \mathbf{0} & \phi_{3}(\mathbf{P}) & \mathbf{0} \\
\mathbf{0} & \phi_{1}(\mathbf{P}) & \mathbf{0} & \phi_{2}(\mathbf{P}) & \mathbf{0} & \phi_{3}(\mathbf{P})
\end{array}\right]
$$

e $\phi_{\mathrm{i}}$ são as funções interpolação quadrática, já vistas na eq. 2.56 considerando-se a coordenada local homogênea $\boldsymbol{\xi}$. Também neste caso, as eq. $\mathbf{2 . 5 6}$ prevêem o uso de elemento descontínuo.

Reescreve-se a eq.(3.19) considerando-se as eqs.(3.21) na forma: 


$$
\left.\underset{\sim}{\mathbf{c}(Q)} \underset{\sim}{\mathbf{u}}(\mathbf{Q})+\sum_{\mathbf{j}=1}^{\mathbf{N}_{\mathrm{e}}}\left[\underset{\sim}{\mathbf{h}}(\mathbf{Q}) \cdot \underset{\sim}{\mathbf{U}^{\mathbf{N}}}\right]=\sum_{\mathbf{j}=1}^{\mathbf{N}_{\mathrm{e}}}[\underset{\sim \mathbf{j}}{\mathbf{g}}(\mathbf{Q}) \cdot \underset{\sim}{\mathbf{j}}]+\underset{\sim}{\mathbf{N}}\right] \mathbf{t}(\mathbf{Q})
$$

onde

$$
\begin{aligned}
& \underset{\sim j}{\mathbf{h}}(\mathbf{Q})=\int_{\Gamma_{\mathrm{j}}}\left[\mathbf{p}_{\sim}^{*}(\mathbf{Q}, \mathbf{P}) \cdot{\underset{\sim}{\boldsymbol{\Phi}}}^{\mathrm{T}}(\mathbf{P})\right] \mathrm{d} \Gamma_{\mathbf{j}}(\mathbf{P}) \\
& \underset{\sim \mathbf{j}}{\mathbf{g}}(\mathbf{Q})=\int_{\Gamma_{\mathbf{j}}}\left[\mathbf{u}^{*}(\mathbf{Q}, \mathbf{P}) \cdot \underset{\sim}{\boldsymbol{\Phi}}{ }^{\mathrm{T}}(\mathbf{P})\right] \mathrm{d} \Gamma_{\mathbf{j}}(\mathbf{P}) \\
& \left.\underset{\sim}{\mathbf{t}}(\mathbf{Q})=\int_{\Omega_{\mathrm{b}}} \underset{\sim \mathbf{b}}{\mathbf{u}_{\mathbf{b}}^{*}}(\mathbf{Q}, \mathbf{p}) \cdot \underset{\sim}{\mathbf{b}}(\mathbf{p})\right] \mathrm{d} \Omega_{\mathrm{b}}(\mathbf{p})
\end{aligned}
$$

cujas integrais têm valores conhecidos e podem ser resolvidas numericamente.

Da mesma forma que foi feito para o MEC aplicado à flexão de placas, somarse a influência de todos os elementos de contorno no cálculo do deslocamento de todos os nós do contorno da placa, constrói-se o seguinte sistema de equações:

$$
\underset{\sim}{\mathbf{H}}(\mathbf{Q}) \underset{\sim}{\mathbf{U}}=\underset{\sim}{\mathbf{G}}(\mathbf{Q}) \underset{\sim}{\mathbf{P}}+\underset{\sim}{\mathbf{T}}(\mathbf{Q})
$$

onde

$\rightarrow \mathbf{H}(\mathbf{Q})$ e $\mathbf{G}(\mathbf{Q})$ contêm os valores das duas primeiras das eqs.(3.24), respectivamente, agrupadas de acordo com os valores nodais a que se relacionam; $\underset{\sim}{\mathbf{H}}(\mathbf{Q})$ contem inclusive os valores de $\underset{\sim}{\mathbf{c}}(\mathbf{Q})$

$\rightarrow \mathbf{T}(\mathbf{Q})$ refere-se aos valores calculados na última das eqs.(3.24); 


$$
\begin{aligned}
& \rightarrow \underset{\sim}{\mathbf{U}^{\mathrm{T}}}=\left\{\begin{array}{lllll}
\mathbf{u}_{1}^{1} & \mathbf{u}_{2}^{1} & \ldots & \mathbf{u}_{1}^{2} & \mathbf{u}_{2}^{2}
\end{array}\right\} \\
& \rightarrow \underset{\sim}{\mathbf{P}^{\mathrm{T}}}=\left\{\begin{array}{lllll}
\mathbf{p}_{1}^{1} & \mathbf{p}_{2}^{1} & \ldots & \mathbf{p}_{1}^{2} & \mathbf{p}_{2}^{2}
\end{array}\right\}
\end{aligned}
$$

sendo $\mathbf{N}_{\mathrm{n}}$ o número de pontos do contorno.

Pode-se representar o sistema de equações na forma:

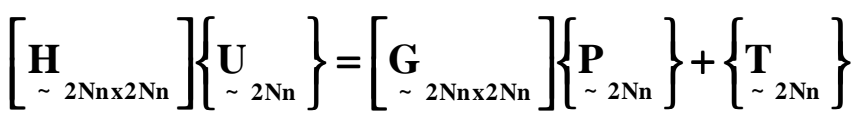

Da mesma forma que para o MEC aplicado ao problema de flexão de placas, partir dos resultados de deslocamentos e forças obtidos pela eq. 3.25, podem-se calcular os deslocamentos de qualquer ponto do contorno ou domínio da placa através das equações eqs. 3.17 e 3.18. Através das relações dos itens 3.4 e $\mathbf{3 . 5}$, calculam-se os esforços e tensões e deformações nestes pontos (ver, p.e., PALERMO(1989)). Novamente, para efeito de programação, estas equações são manipuladas como se fez à eq. 3.18, cujo resultado ilustra-se na eq. 3.25.

Da mesma forma que no problema de placas, pode-se verificar propriedades da matriz $\mathbf{H}$ através da imposição de movimento de corpo rígido ã chapa. Supondo este deslocamento dado na direção $\mathbf{x}_{\mathbf{1}}$ :

$$
\sum_{j=1}^{N n} h_{i, 2 j-1}=0
$$

ou seja, deve ser nula a soma entre os valores das colunas ímpares de $\underset{\sim}{\mathbf{H}}$. O mesmo vale para as colunas pares quando o deslocamento de corpo rígido se dá na direção de $\mathbf{x}_{2}$ :

$$
\sum_{j=1}^{N n} h_{i, 2 j}=0
$$


Para a integração sobre os elementos na eq. 3.24, procede-se da mesma forma como foi feito para o caso de flexão de placas (cap. 2), lembrando-se que, os valores fundamentais em $\mathbf{p}^{*}(\mathbf{Q}, \mathbf{P})$ e $\underset{\sim}{\mathbf{u}^{*}}(\mathbf{Q}, \mathbf{P})$ agora são os dados em eq. 3.13 e 3.14. Neste trabalho, como se disse, não haverá a necessidade de cálculo de integrais singulares, pois as equações foram todas escritas para nós externos ao domínio da placa. Utilizou-se também para esta análise de chapas, a técnica do subelemento para efeito da integração sobre elementos de contorno. 


\section{CAPÍTULO 4-ELEMENTO DE BARRA MODELADO PELO MÉTODO DOS ELEMENTOS FINITOS}

\section{1 - INTRODUÇÃO}

No presente capítulo apresentam-se aspectos sobre o elemento finito de barra utilizado para discretizar os elementos estruturais lineares. No entanto, apenas os principais aspectos para efeito de uniformização e as convenções de sinais relativos aos sistemas locais e globais de coordenadas. Isto visa facilitar a compreensão do processo de acoplamento destes com os elementos estruturais de superfícies modelados pelo MEC que se verá adiante. Esta breve recapitulação sobre estes aspectos se deve ao fato deste ser um método bastante conhecido, estudado e divulgado na ampla bibliografia sobre o assunto (ver, p.e., BATHE(1982), ZIENKEWICZ(1971), CODA(1993), RAMALHO(1990)).

Um dos citados aspectos diz respeito ao vetor de carga que originalmente, não se apresentam em termos de valores nodais, como ocorre no MEC. E ainda, a 
orientação dos sistemas de coordenadas locais e globais destas estruturas serão estabelecidos. Deve-se atentar para uma adaptação e possível ampliação universo de graus de liberdade dos elementos de superfície para efeito do acoplamento com barras (CODA (1993)), como se verá no cap. 6.

Um outro aspecto se refere à orientação do elemento de barra. Esta análise auxiliará na correta elaboração da entrada de dados, discretizando-o corretamente.

\section{2 - MATRIZ DE TRANSFORMAÇÃO DO VETOR DE FORÇA PARA VIABILIZAR O ACOPLAMENTO}

Para converter o vetor de forças nodais em tensões e cargas distribuídas no contorno, é necessário determinar-se a matriz de transformação aqui denominada matriz C. Este procedimento se encontra bem detalhado em CODA(1993).

Para o elemento de barra adotado, sabe-se que, a partir da aplicação do Princípio dos Trabalhos Virtuais (PTV) determina-se a equação de equilibro de corpos elásticos pode ser escrita na forma matricial como:

$$
\mathbf{K} \mathbf{U}=\mathbf{F}
$$

onde $\mathbf{K}$ é a matriz de rigidez do corpo definido por pontos discretos, $\mathbf{U}$ o vetor de deslocamentos destes pontos e $\mathbf{F}$ o vetor de forças aplicadas representadas por componentes nodais aplicadas aos nós do corpo.

As matrizes componentes desta equação poderão sofrer modificações, seja de transformação de coordenadas, seja conforme outras formas em que elas podem ser escritas, para que se proceda ao acoplamento com o corpo modelado pelo MEC, conforme se segue. 


\subsection{1 - A MATRIZ C}

Considere-se o elemento de comprimento $\mathbf{L}$ e valores nodais como demonstrados na fig. 4.1. Será analisada a variação do deslocamento ao longo do vão deste elemento. Primeiramente, será calculada a forma aproximada do deslocamento vertical $(\boldsymbol{\omega})$ ao longo do eixo $\mathbf{X}_{\mathbf{1}}$. Sendo considerada uma aproximação polinomial da função $\omega$, pode-se escrever:

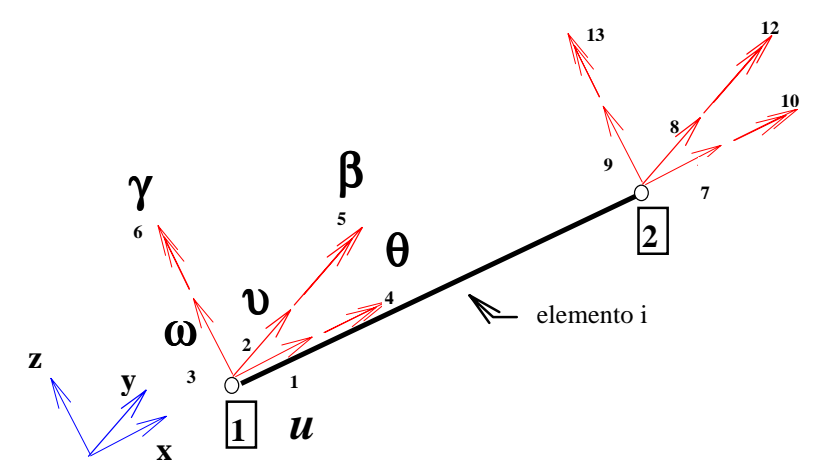

Fig. 4.1 - Coordenadas locais do elemento de barra

$$
\omega_{a}(x)=D_{0} x^{3}+C_{0} x^{2}+B_{0} x+A
$$

que em termos de coordenadas homogêneas fica:

$$
\omega_{\mathrm{a}}(\xi)=D \xi^{3}+C \xi^{2}+B \xi+A
$$

uma vez que

$$
\xi=\mathbf{x} / \mathbf{L}
$$

e sendo

$$
\begin{aligned}
& D=D_{0} L^{3} \\
& C=C_{0} L^{2} \\
& B=B_{0} L
\end{aligned}
$$

$\mathrm{e}$ 
$\omega_{\mathrm{a}}(\xi)$ é o valor aproximado de $\boldsymbol{\omega}$ no nó de posição $\xi$.

A primeira derivada da eq. $\mathbf{4 . 3}$ relação à coordenada $\mathbf{x}$ é

$$
\omega_{\mathrm{a}}^{\prime}(\xi)=\left(3 D \xi^{2}+2 C \xi+B\right) \frac{1}{L}=-\beta_{a}(\xi)
$$

uma vez que

$$
\omega_{\mathrm{a}}^{\prime}(\xi)=\frac{\partial \omega_{\mathrm{a}}(\xi)}{\partial \mathrm{x}}=\frac{\partial \omega_{\mathrm{a}}(\xi)}{\partial \xi} \cdot \frac{\partial \xi}{\partial \mathbf{x}}
$$

e

$$
\frac{\partial \omega_{\mathrm{a}}(\xi)}{\partial \xi}<0
$$

Os coeficientes constantes $\mathbf{A}, \mathbf{B}, \mathbf{C}$ and $\mathbf{D}$ podem ser calculados ao se impor as condições de contorno às eqs. $\mathbf{4 . 3}$ e 4.4 da forma:
a) $\operatorname{para} x=0(\Rightarrow \xi=0) ; \omega_{\mathbf{a}}(\mathbf{0})=\omega_{1}$
b) $\operatorname{para} x=0(\Rightarrow \xi=0) ; \omega^{\prime}(0)=\beta_{1}$
c) $\operatorname{para} \mathrm{x}=\mathrm{L}(\Rightarrow \xi=1) ; \boldsymbol{\omega}_{\mathrm{a}}(\mathbf{1})=\omega_{2}$
d) $\operatorname{para} x=0(\Rightarrow \xi=1) ; \omega^{\prime}{ }_{a}(\mathbf{1})=\beta_{2}$

o que dá o seguinte sistema de equações:

$$
\begin{aligned}
& A=\omega_{1} \\
& B=-\beta_{1} L \\
& A+B+C+D=\omega_{2} \\
& B+2 C+3 D=-\beta_{2} L
\end{aligned}
$$

que resolvido transforma as eqs. 4.3 e $4.4 \mathrm{em}$ : 


$$
\begin{aligned}
& \omega_{\mathrm{a}}(\xi)=\omega_{1} \varphi_{1}-\beta_{1} \varphi_{2}+\omega_{2} \varphi_{3}-\beta_{2} \varphi_{4} \\
& -\beta_{\mathrm{a}}(\xi)=\omega_{1} \varphi_{1}^{\prime}-\beta_{1} \varphi_{2}^{\prime}+\omega_{2} \varphi_{3}^{\prime}-\beta_{2} \varphi_{4}^{\prime}
\end{aligned}
$$

onde

$$
\begin{array}{lll}
\varphi_{1}=\left(1-3 \xi^{2}+2 \xi^{3}\right) & \varphi_{1}^{\prime}=\left(-6 \xi+6 \xi^{2}\right) / L \\
\varphi_{2}=\left(\xi-2 \xi^{2}+\xi^{3}\right) / L & \text { e } & \varphi^{\prime}{ }_{2}=\left(1-4 \xi+3 \xi^{2}\right) \\
\varphi_{3}=\left(3 \xi^{2}-2 \xi^{3}\right) & & \varphi^{\prime}{ }_{3}=\left(6 \xi-6 \xi^{2}\right) / L \\
\varphi_{4}=\left(-\xi^{2}+\xi^{3}\right) / L & & \varphi^{\prime}{ }_{4}=\left(-2 \xi+3 \xi^{2}\right)
\end{array}
$$

O mesmo procedimento pode ser feito para se obter a equação aproximada dos deslocamentos $\boldsymbol{v}$ em termos de coordenadas homogêneas e valores nodais, dando:

$$
\begin{aligned}
& v_{\mathrm{a}}(\xi)=v_{1} \varphi_{1}+\gamma_{1} \varphi_{2}+v_{2} \varphi_{3}+\gamma_{2} \varphi_{4} \\
& v_{\mathrm{a}}^{\prime}(\xi)=v_{1} \varphi_{1}^{\prime}+\gamma_{1} \varphi_{2}^{\prime}+v_{2} \varphi_{3}^{\prime}+\gamma_{2} \varphi_{4}^{\prime}=\gamma_{\mathrm{a}}(\xi)
\end{aligned}
$$

uma vez que $\frac{\partial v_{a}(\xi)}{\partial \xi}>\mathbf{0}$ pois ambos os eixos $\mathbf{X}_{\mathbf{3}}$ e $\mathbf{X}_{\mathbf{2}}$ são eixos de flexão. Com relação ao eixo $\mathbf{X}_{\mathbf{1}}$, entretanto, a forma aproximada para $\boldsymbol{u}$ e $\boldsymbol{\alpha}$ deve ser linear (pois existem somente 2 valores nodais em cada nó). Daí vem que:

$$
u_{\mathrm{a}}(\mathbf{x})=\mathbf{E}_{\mathbf{o}} \mathbf{x}+\mathbf{F}
$$

que em coordenadas homogêneas fica:

$$
u_{\mathrm{a}}(\xi)=\mathbf{E} \xi+\mathbf{F}
$$

sendo

$$
\mathbf{E}=\mathbf{E}_{\mathbf{0}} \mathbf{L}
$$


Novamente, impondo-se as condições de contorno à eq. 4.12:
a) $\operatorname{para} \mathbf{x}=\mathbf{0}(\Rightarrow \xi=0) ; u_{\mathrm{a}}(\mathbf{0})=\boldsymbol{u}_{1}$
b) $\operatorname{para} x=\mathbf{L}(\Rightarrow \xi=\mathbf{1}) ; \boldsymbol{u}_{\mathrm{a}}(\mathbf{1})=\boldsymbol{u}_{\mathbf{2}}$

Resultando no sistema de equações:

$$
\begin{aligned}
& \mathrm{F}=u_{1} \\
& \mathrm{E}=\boldsymbol{u}_{2}-u_{1}
\end{aligned}
$$

Transformando a eq. $\mathbf{4 . 1 2}$ em:

$$
\begin{aligned}
& u_{\mathrm{a}}(\xi)=(1-\xi) u_{1}+(\xi) u_{2} \quad \text { ou } \\
& u_{\mathrm{a}}(\xi)=\theta_{1} u_{1}+\theta_{2} u_{2}
\end{aligned}
$$

sendo

$$
\begin{aligned}
& \theta_{1}=(1-\xi) \quad \mathrm{e} \\
& \theta_{2}=\xi
\end{aligned}
$$

Analogamente, portanto, obtém-se:

$$
\alpha_{\mathrm{a}}(\xi)=\theta_{1} \alpha_{1}+\theta_{2} \alpha_{2}
$$

Agrupando-se as eqs. 4.8, 4.10, 4.15 e 4.17, pode-se dizer que a função interpolação para os deslocamentos é: 


$$
\Psi=\left[\begin{array}{cccccccccccc}
\theta_{1} & 0 & 0 & 0 & 0 & 0 & \theta_{2} & 0 & 0 & 0 & 0 & 0 \\
0 & \varphi_{1} & 0 & 0 & 0 & \varphi_{2} & 0 & \varphi_{3} & 0 & 0 & 0 & \varphi_{4} \\
0 & 0 & \varphi_{1} & 0 & -\varphi_{2} & 0 & 0 & 0 & \varphi_{3} & 0 & -\varphi_{4} & 0 \\
0 & 0 & 0 & \theta_{1} & 0 & 0 & 0 & 0 & 0 & \theta_{2} & 0 & 0 \\
0 & 0 & -\varphi_{1}^{\prime} & 0 & \varphi^{\prime}{ }_{2} & 0 & 0 & 0 & -\varphi^{\prime} & 0 & \varphi^{\prime} & 0 \\
0 & \varphi^{\prime} & 0 & 0 & 0 & \varphi^{\prime} & 0 & \varphi^{\prime} & 0 & 0 & 0 & \varphi^{\prime} \\
\end{array}\right]_{6 \times 12}
$$

pois

$$
\left\{\begin{array}{c}
u_{\mathrm{a}}(\xi) \\
v_{\mathrm{a}}(\xi) \\
\omega_{\mathrm{a}}(\xi) \\
\alpha_{\mathrm{a}}(\xi) \\
\beta_{\mathrm{a}}(\xi) \\
\gamma_{\mathrm{a}}(\xi)
\end{array}\right\}_{6 \times 1}=\Psi\left\{\begin{array}{c}
u_{1} \\
v_{1} \\
\omega_{1} \\
\alpha_{1} \\
\beta_{1} \\
\gamma_{1} \\
u_{2} \\
v_{2} \\
\omega_{2} \\
\alpha_{2} \\
\beta_{2} \\
\gamma_{2}
\end{array}\right\}_{12 \times 1}
$$

$\mathrm{Na}$ formulação MEF que gerou a eq. 4.1, porém o termo relacionado ao carregamento distribuído é escrito em sua forma integral como (fig. 4.2):

$$
\int_{0}^{L_{i}} \varphi(x) \cdot P(x) d x=L_{i} \int_{0}^{1} \varphi(\xi) \cdot P(\xi) d \xi
$$

definida sobre o comprimento do elemento $\mathbf{i}$. 


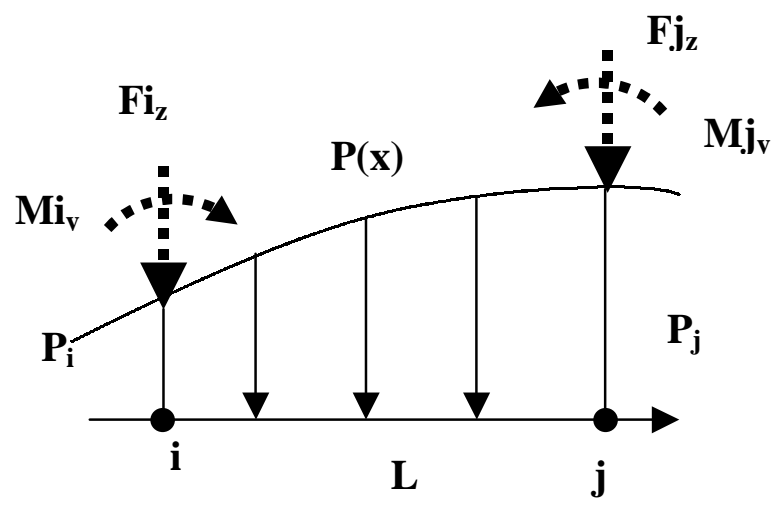

Fig. 4.2 - Cálculo de forças equivalentes

$\mathbf{P}(\mathbf{x})$ é o carregamento aplicado ao elemento i que varia ao longo do seu vão e de valores interpolado por $\boldsymbol{\varphi}$. Pode-se interpolar estes valores nos nós extremos do elemento, com o auxílio de funções lineares como as utilizadas na aproximação de deslocamentos nodais.

$$
\sim \underset{P}{\sim}\left\{\begin{array}{c}
\mathbf{P}_{\mathrm{aX}}(\xi) \\
\mathbf{P}_{\mathrm{aY}}(\xi) \\
\mathbf{P}_{\mathrm{aZ}}(\xi) \\
\mathbf{M}_{\mathrm{aX}}(\xi) \\
\mathbf{M}_{\mathrm{aY}}(\xi) \\
\mathbf{M}_{\mathrm{aZ}}(\xi)
\end{array}\right\}_{6 \mathrm{x} 1} \sim\left\{\begin{array}{c}
\mathbf{P}_{1 \mathrm{X}} \\
\mathbf{P}_{1 \mathrm{Y}} \\
\mathbf{P}_{1 \mathrm{Z}} \\
\mathbf{M}_{1 \mathrm{X}} \\
\mathbf{M}_{1 \mathrm{Y}} \\
\mathbf{M}_{1 \mathrm{Z}} \\
\mathbf{P}_{2 \mathrm{X}} \\
\mathbf{P}_{2 \mathrm{Y}} \\
\mathbf{P}_{2 \mathrm{Z}} \\
\mathbf{M}_{2 \mathrm{X}} \\
\mathbf{M}_{2 \mathrm{Y}} \\
\mathbf{M}_{2 \mathrm{Z}}
\end{array}\right\}_{12 \mathrm{x} 1} \sim \mathbf{P}
$$

onde 


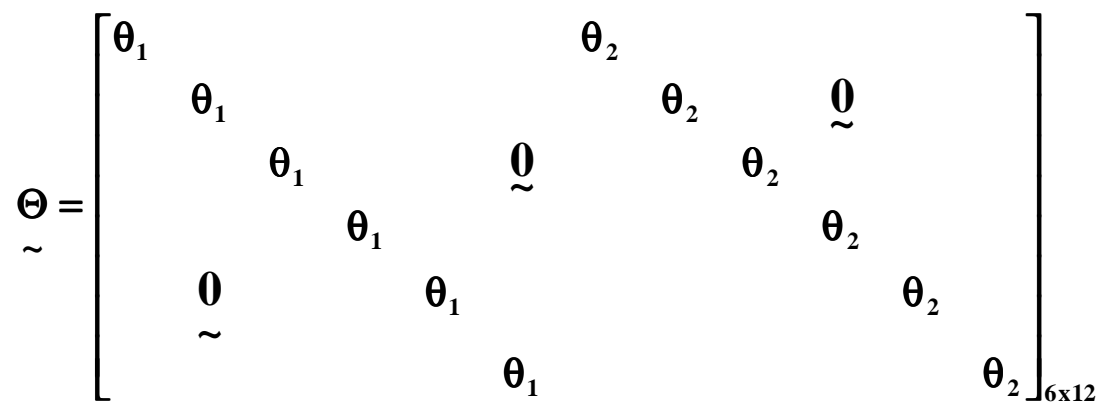

$\mathrm{Na}$ forma matricial, eq. 4.20 fica:

$$
\mathbf{L}_{\mathrm{i}} \int_{0}^{1} \varphi(\xi) \cdot \mathbf{P}(\xi) \mathrm{d} \xi=\mathbf{L}_{\mathrm{i}} \int_{0}^{1} \underset{\sim}{\Psi^{\mathrm{T}}} \underset{\sim}{\Theta} \underset{\sim}{\mathbf{P}} \mathbf{d} \xi=\mathbf{L}_{\mathrm{i}}\left[\int_{0}^{1} \underset{\sim}{\Psi^{\mathrm{T}}} \underset{\sim}{\Theta} \mathrm{d} \xi\right] \underset{\sim}{\mathbf{P}}
$$

sendo $\underset{\sim}{\Psi}$ dado pela eq. $\mathbf{4 . 1 8}$

O vetor de forças nodais pode ser substituído pela expressão 4.23, isto é:

$$
\mathbf{F}=\mathbf{C P}
$$

onde $\mathbf{P}$ pode ser obtido de eq. 4.21 e: 


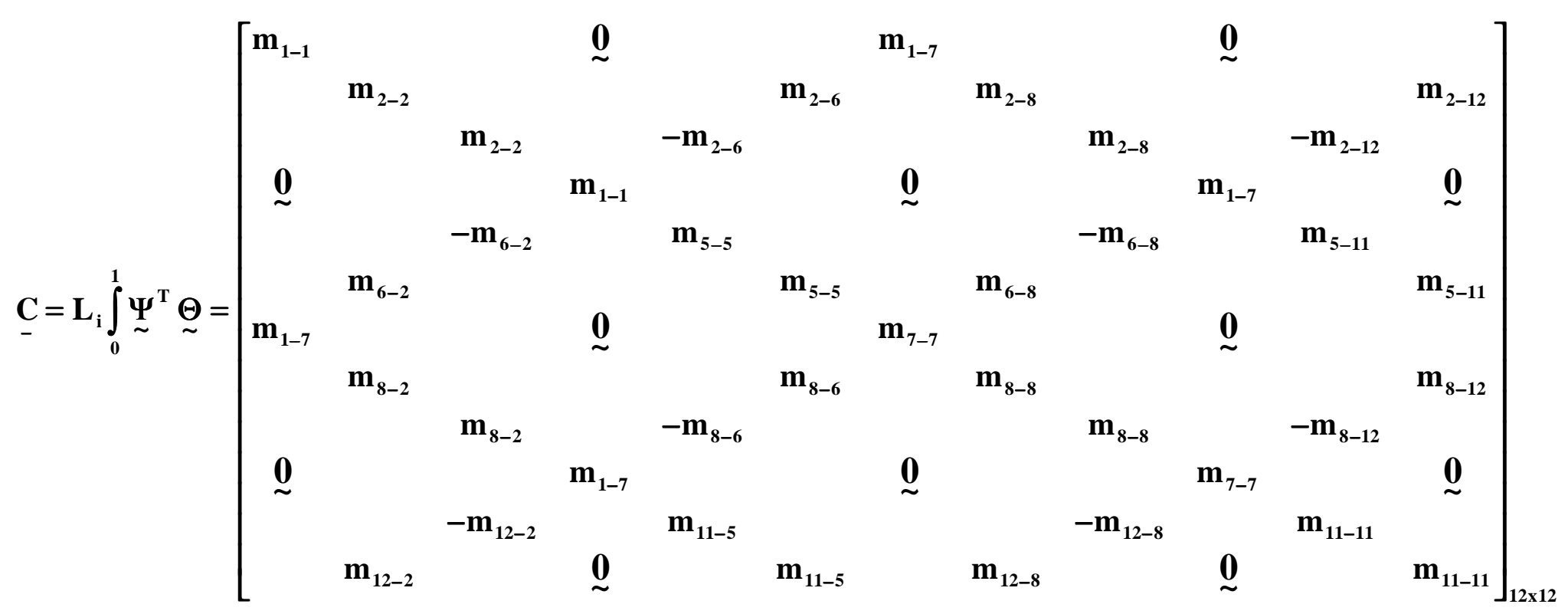

onde

$$
\begin{aligned}
& \mathbf{m}_{1-1}=L_{i} \int_{0}^{1} \theta_{1}{ }^{2} d \xi=L_{i} / 3 \\
& m_{2-2}=L_{i} \int_{0}^{1} \varphi_{1} \theta_{1} d \xi=7 L_{i} / 20
\end{aligned}
$$




$$
\begin{aligned}
& \mathbf{m}_{5-5}=L_{\mathrm{i}} \int_{0}^{1} \varphi_{2}^{\prime} \theta_{1} d \xi=L_{i} / 12 \\
& \mathbf{m}_{7-7}=\mathbf{L}_{\mathrm{i}} \int_{0}^{1} \theta_{2}{ }^{2} \mathbf{d} \xi=\mathrm{L}_{\mathrm{i}} / 3 \\
& m_{8-8}=L_{i} \int_{0}^{1} \varphi_{3} \theta_{2} d \xi=7 L_{i} / 20 \\
& m_{11-11}=L_{i} \int_{0}^{1} \varphi^{\prime}{ }_{4} \theta_{2} d \xi=L_{i} / 12 \\
& m_{2-6}=L_{i} \int_{0}^{1} \varphi_{1}^{\prime} \theta_{1} d \xi=-1 / 2 \\
& \mathbf{m}_{1-7}=L_{\mathrm{i}} \int_{0}^{1} \theta_{1} \theta_{2} d \xi=L_{\mathrm{i}} / 6 \\
& m_{2-8}=L_{i} \int_{0}^{1} \varphi_{1} \theta_{2} d \xi=3 L_{i} / 20 \\
& m_{2-12}=L_{i} \int_{0}^{1} \varphi_{1}^{\prime} \theta_{2} d \xi=-1 / 2 \\
& \mathbf{m}_{6-2}=L_{i} \int_{0}^{1} \varphi_{2} \theta_{1} d \xi=L_{i}^{2} / 20 \\
& \mathbf{m}_{6-8}=L_{i} \int_{0}^{1} \varphi_{2} \theta_{2} d \xi=L_{i}^{2} / 30 \\
& m_{5-11}=L_{i} \int_{0}^{1} \varphi^{\prime}{ }_{2} \theta_{2} d \xi=-L_{i} / 12 \\
& m_{8-2}=L_{i} \int_{0}^{1} \varphi_{3} \theta_{1} d \xi=3 L_{i} / 20 \\
& m_{8-6}=L_{i} \int_{0}^{1} \varphi^{\prime}{ }_{3} \theta_{1} d \xi=-1 / 2 \\
& \mathbf{m}_{8-12}=L_{i} \int_{0}^{1} \varphi^{\prime}{ }_{3} \theta_{2} d \xi=-1 / 2 \\
& m_{12-2}=L_{i} \int_{0}^{1} \varphi_{4} \theta_{1} d \xi=-L_{i}^{2} / 30
\end{aligned}
$$




$$
\begin{aligned}
& m_{11-5}=L_{i} \int_{0}^{1} \varphi^{\prime}{ }_{4} \theta_{1} d \xi=-L_{i} / 12 \\
& m_{12-8}=L_{i} \int_{0}^{1} \varphi_{4} \theta_{2} d \xi=-L_{i}^{2} / 20
\end{aligned}
$$

(4.26)cont.

Observa-se que a matriz $\mathbf{C}_{-}$não é simétrica.

Um outro aspecto desta matriz deve ser considerado no momento do acoplamento da barra com outros elementos estruturais modelados por outros métodos numéricos, como o MEC, por exemplo. Neste caso o vetor $\mathbf{C P}$ é interpretado como contendo os valores nodais de componentes força de superfície. $\mathrm{O}$ exemplo da barra da fig. 4.3 ilustra bem este problema. Compondo-se a matriz conforme foi elaborado acima, o resultado seria interpretado como se interação entre as barras fosse refletida ao longo do vão do segundo trecho dela.
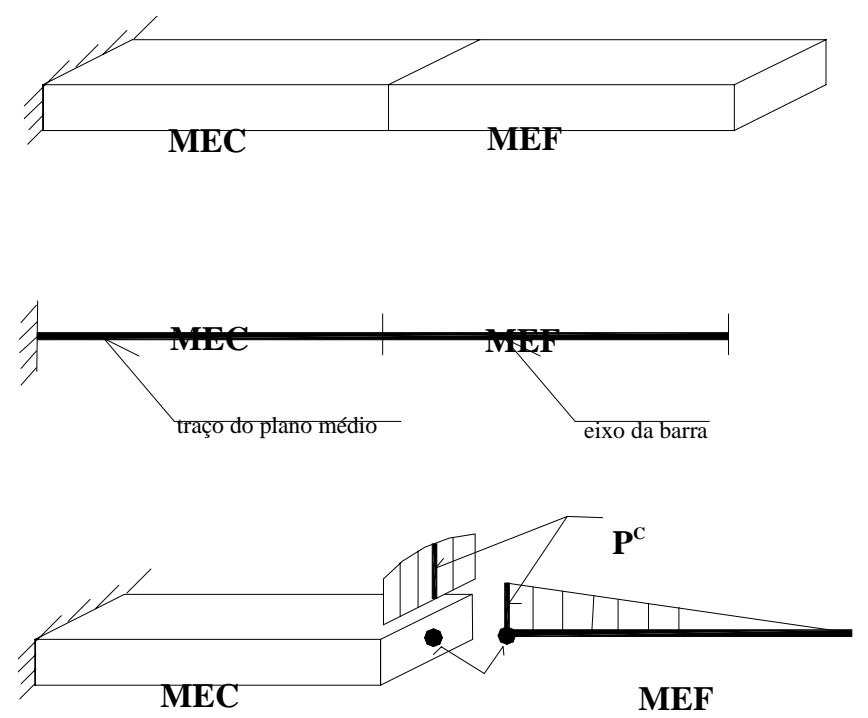

Fig. 4.3 - Ligação entre barras modeladas pelo MEC e MEF

Uma solução seria escrever a matriz $\mathbf{C}$ a partir da matriz elementar de equilíbrio (eq. 4.1 escrita para o elemento) ou utilizar apoios discretos no ponto de ligação e suprimir a segunda barra (laje cogumelo, p.e., os pilares podem ser interpretados tanto como apoios rígidos na direção vertical e flexíveis às rotações) ou 
pode-se utilizar o modelo da fig. 4.4, adotado por CODA \& VENTURINI(1999) para uma viga em balanço como a da fig. 4.3.

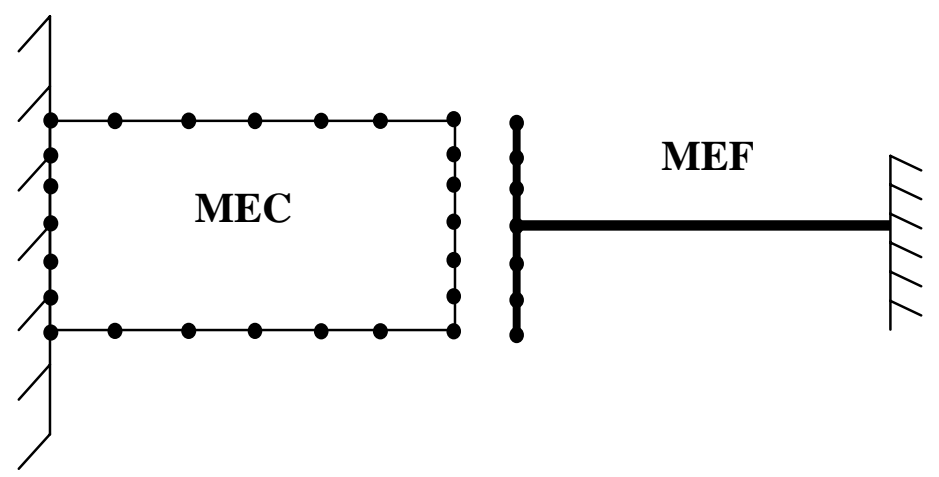

Fig. 4.4 - Modelo adotado por CODA \& VENTURINI (1999)

\subsection{2 - ORIENTAÇÃO DAS BARRAS - MATRIZ DE TRANSFORMAÇÃO DE COORDENADAS}

Precisa-se, agora, escrever a matriz $\mathbf{C}$ associada às coordenadas globais para efeito de consideração do elemento associado a outros elementos estruturais. Sabe-se que a matriz de transformação entre os sistemas locais e globais :

$$
\underset{\sim}{\mathbf{T}_{\mathrm{e}}^{\mathrm{i}}}=\left[\begin{array}{lll}
\mathrm{l}_{1} & \mathrm{~m}_{1} & \mathbf{n}_{1} \\
\mathrm{l}_{2} & \mathrm{~m}_{2} & \mathrm{n}_{2} \\
\mathrm{l}_{3} & \mathrm{~m}_{3} & \mathrm{n}_{3}
\end{array}\right]
$$

sendo $\mathbf{l}_{\mathbf{k}}, \mathbf{m}_{\mathbf{k}}$ e $\mathbf{n}_{\mathbf{k}}$ cossenos diretores das coordenadas locais em relação às globais $\mathbf{X}_{\mathbf{K}}$. Podem ser determinados seguindo-se o esquema da fig. 4.5. 

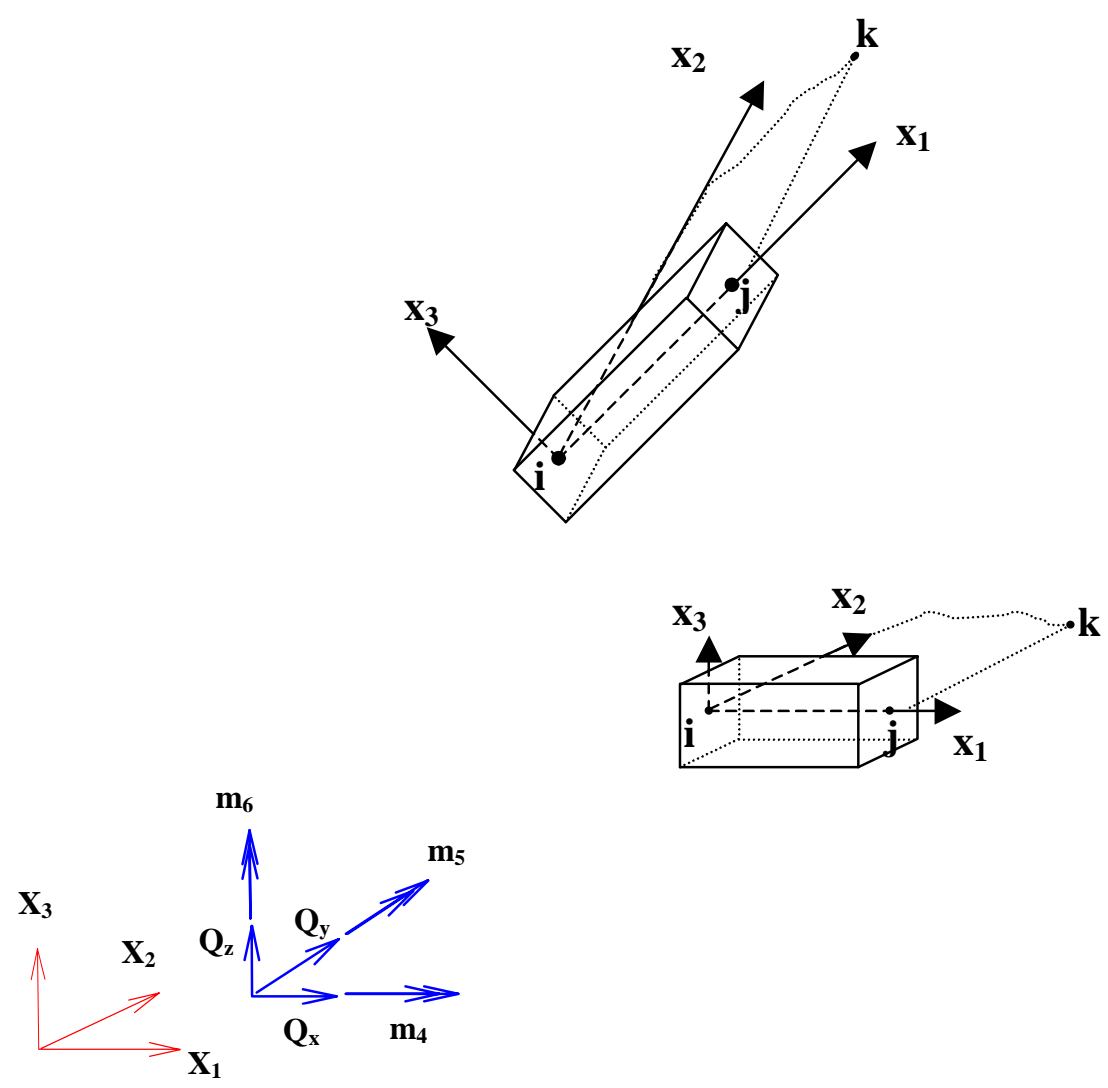

Fig. 4.5 - Sistemas de coordenadas locais e globais do elemento de barra

Para o elemento de barra, portanto, a transformação das coordenadas locais para globais será feita através da matriz (fig. 4.6):

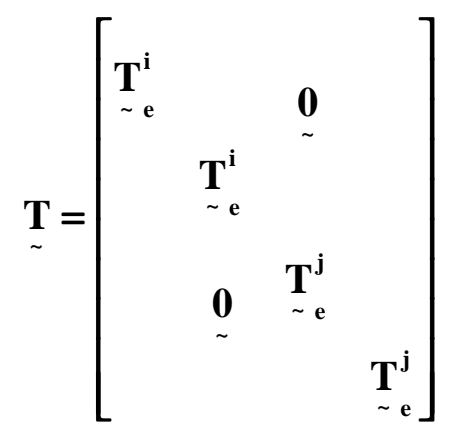

Portanto, pode-se dizer que o vetor de forças pode ser transformado do sistema local para global por:

$$
\mathbf{F}=\mathbf{T}^{\mathrm{T}} \mathbf{F}_{\mathrm{e}}=\mathbf{T}^{\mathrm{T}}\left(\mathbf{C}_{\mathrm{e}} \mathbf{P}_{\mathrm{e}}\right)
$$




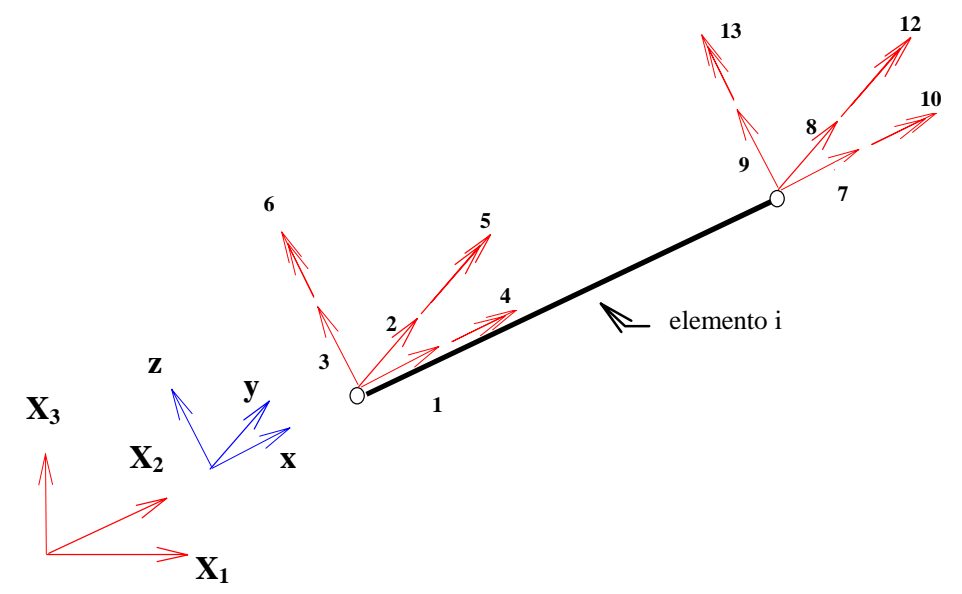

Fig. 4.6 - Sistemas de coordenadas locais e globais do elemento de barra

Como, de forma análoga:

$$
\mathbf{P}=\mathbf{T}^{\mathbf{T}} \mathbf{P}_{\mathrm{e}}
$$

a eq. 4.28 pode ser reescrita como:

$$
\begin{aligned}
& \mathbf{F}=\mathbf{T}^{\mathrm{T}}\left(\mathrm{C}_{\mathrm{e}} \mathbf{T P}\right) \\
& \sim \underbrace{\sim \sim \sim}_{\mathrm{C}} \sim
\end{aligned}
$$

de onde obtém-se

$$
\underset{\sim}{\mathbf{C}}=\mathbf{T}_{\sim}^{\mathbf{T}} \mathbf{C}_{\mathbf{e}} \mathbf{T}
$$




\section{CAPÍTULO 5 - TÉCNICA DAS SUBREGIÕES}

\section{1 - INTRODUÇÃO}

Esta técnica consiste em dividir um domínio em partes que agrupem propriedades homogêneas e aplicar o MEC ou MEF a cada sub-região assim definida. Então, os subsistemas de equações obtidos para cada subdomínio são agrupados em um único para se atingir o resultado requerido. Este é o exatamente o caso de pavimentos de edifícios usuais, considerando-se, não apenas o material de que são compostos, mas principalmente a variação de espessura (t) que ocorre facilmente nos diversos casos.

Ainda, no caso de sistemas estrutural placa-viga, mesmo quando não há variação entre as espessuras das placas sobre o pavimento, surgem vinculações no domínio do mesmo que devem ser levadas em conta. Portanto, o fato desta técnica facilitar esta consideração a torna uma ferramenta muito útil. Além disso, ela provoca o surgimento de blocos de zero no sistema final de equações que representa o comportamento do domínio. VENTURINI(1987) aponta como vantagens para este 
fato, não ser necessário armazenar estes blocos e a redução número de operações durante a resolução do sistema, como se verá mais tarde neste trabalho.

A técnica das sub-regiões é bastante conhecida, portanto aqui serão expostos o aspecto computacional e as modificações a serem feitos em casos especiais. ALIABADI(1991), CODA(1993), VENTURINI(1987, 1983), PALERMO(1989), BREBBIA\&GEOUGIOU(1979), KOMATSU \& al(1997), WEARING \& BETTAHAR (1994) e muitos outros, resolveram seus problemas em estudo através da técnica das sub-regiões.

A divisão do domínio da placa em estudo em sub-regiões, porém, deve ser feita quando necessário e critério (VENTURINI(1987)), a introdução da descontinuidade onde ela inexiste, provoca alterações nos resultados calculados (vide tab.5.1).

\section{2 - FORMULAÇÃO BÁSICA}

Do que se estudou sobre o MEC aplicado às placas de Kirchhoff, torna-se necessário obter a formulação para placas divididas em várias sub-regiões, também conhecida como formulação multi-domínio ("multi-domain formulation" ALIABADI(1991)). Para elaborá-la, o seguinte exemplo de uma placa dividida em três sub-regiões será utilizado (fig.5.1):

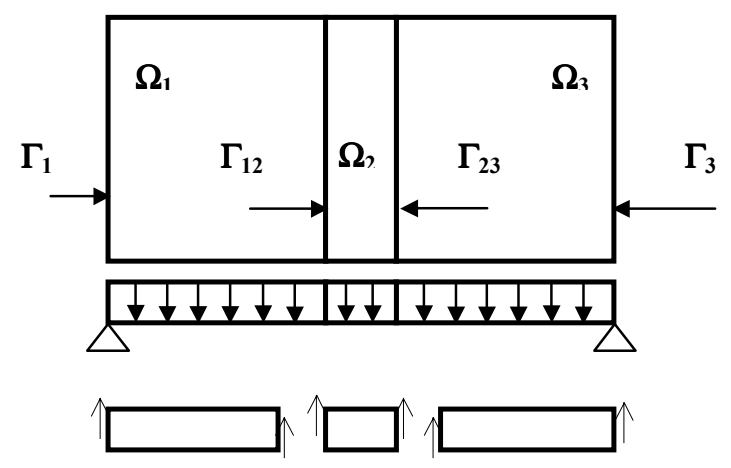

Fig. 5.1 - Exemplo para placa apoiada dividida em três subdomínios 
Nesta figura, sendo aqui $\mathbf{i}, \mathbf{j}=1,3$,

$\rightarrow \Gamma^{\mathrm{i}}$ contorno da região $\Omega^{\mathrm{i}}$ que não pertence a uma interface (contorno comum às duas placas)

$\rightarrow \Gamma^{\mathrm{ij}}$ contorno da interface entre as sub-regiões $\Omega^{\mathrm{i}}$ e $\Omega^{\mathrm{j}}$.

Como visto em anteriormente, a representação matricial para único domínio é:

$$
[\mathbf{H}]\{\mathbf{U}\}=[\mathbf{G}]\{\mathbf{P}\}+\{\mathbf{T}\}
$$

Então, esta equação toma a seguinte forma, considerando-se a matriz global particionada de acordo com a subdivisão do domínio neste exemplo específico como:

$$
\left[\begin{array}{ll}
H^{1} & \mathbf{H}^{12}
\end{array}\right]\left\{\begin{array}{c}
\mathbf{U}^{1} \\
\mathbf{U}^{12}
\end{array}\right\}=\left[\begin{array}{ll}
\mathbf{G}^{1} & \mathbf{G}^{12}
\end{array}\right]\left\{\begin{array}{c}
\mathbf{P}^{1} \\
\mathbf{P}^{12}
\end{array}\right\}+\left\{\mathrm{T}^{1}\right\}
$$

para o subdomínio $\mathbf{\Omega}^{1}$,

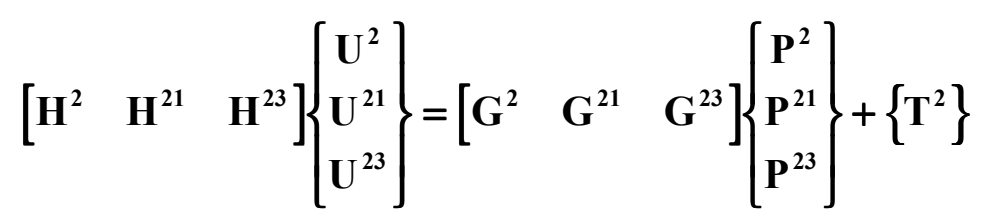

para o subdomínio $\boldsymbol{\Omega}^{2}$,

$$
\left[\begin{array}{ll}
\mathbf{H}^{3} & \mathbf{H}^{32}
\end{array}\right]\left\{\begin{array}{c}
\mathbf{U}^{3} \\
\mathbf{U}^{32}
\end{array}\right\}=\left[\begin{array}{ll}
\mathbf{G}^{3} & \mathbf{G}^{32}
\end{array}\right]\left\{\begin{array}{c}
\mathbf{P}^{3} \\
\mathbf{P}^{32}
\end{array}\right\}+\left\{\mathbf{T}^{3}\right\}
$$

para o subdomínio $\Omega^{3}$, onde

$\rightarrow \mathbf{U}^{\mathrm{i}}, \mathbf{P}^{\mathrm{i}}$ são os deslocamentos e forças de superfície nodais nos pontos do contorno $\Gamma^{\mathrm{i}}$ da sub-região $\boldsymbol{\Omega}^{\mathrm{i}}$.

$\rightarrow \mathbf{U}^{\mathrm{ij}}, \mathbf{P}^{\mathrm{ij}}$ são os deslocamentos e forças de superfície nodais nos pontos do contorno $\Gamma^{\mathrm{ij}}$ da sub-região $\boldsymbol{\Omega}^{\mathrm{i}}$.

$\rightarrow \mathbf{H}^{\mathrm{i}}, \mathbf{G}^{\mathrm{i}}$ são coeficientes das matrizes $\mathbf{H}$ e $\mathbf{G}$ calculados para a sub-região $\boldsymbol{\Omega}^{\mathrm{i}}$ que multiplicam $\mathbf{U}^{\mathrm{i}}$ e $\mathbf{P}^{\mathrm{i}}$ respectivamente.

$\rightarrow \mathbf{H}^{\mathrm{ij}}, \mathbf{G}^{\mathrm{ij}}$ são coeficientes das matrizes $\mathbf{H}$ e $\mathbf{G}$ calculados para a sub-região $\boldsymbol{\Omega}^{\mathrm{i}}$ que multiplicam $\mathbf{U}^{\mathrm{ij}}$ e $\mathbf{P}^{\mathrm{ij}}$ respectivamente.

$\rightarrow \mathbf{T}^{\mathrm{i}}$ são os termos de $\{\mathbf{T}\}$ relativos à sub-região $\Omega^{\mathrm{i}}$. 
Este resultado corresponde alternativamente à aplicação da eq.(5.1) a cada subdomínio separadamente.

Como ambos os deslocamentos e forças de superfície de nós locados sobre uma interface são valores incógnitos, os sistemas de equações mostrados nas equações (eqs. 5.2 a 5.4) não são suficientes para resolver o problema. Torna-se necessário, portanto, obter-se 4 grupos de equações adicionais uma vez que existem quatro novos grupos de incógnitas $\left(\mathbf{U}^{12}\right.$ ou $\mathbf{P}^{12} ; \mathbf{U}^{21}$ ou $\mathbf{P}^{21} ; \mathbf{U}^{23}$ ou $\mathbf{P}^{23} ; \mathbf{U}^{32}$ ou $\left.\mathbf{P}^{32}\right)$. Considerando que as sub-regiões $\mathbf{i}$ e $\mathbf{j}$ exercem uma relação entre si ao longo da interface $\Gamma^{\mathrm{ij}}$, podese escrever $\mathbf{U}^{\mathrm{ij}}$ como uma combinação de $\mathbf{U}^{\mathrm{ji}}$ o mesmo valendo para $\mathbf{P}^{\mathrm{ij}}$ relativamente a $\mathbf{P}^{\mathrm{ji}}$. Este fato é obtido através das condições de equilíbrio e compatibilidade de deslocamentos:

$$
\begin{aligned}
& \mathbf{U}^{12}=\mathbf{U}^{21} \\
& \mathbf{U}^{23}=\mathbf{U}^{32} \\
& \mathbf{P}^{12}=-\mathbf{P}^{21} \\
& \mathbf{P}^{23}=-\mathbf{P}^{32}
\end{aligned}
$$

donde a eq.(5.2) fica:

$$
\left[\begin{array}{ll}
\mathbf{H}^{1} & \mathbf{H}^{12}
\end{array}\right]\left\{\begin{array}{c}
\mathbf{U}^{1} \\
\mathbf{U}^{21}
\end{array}\right\}=\left[\begin{array}{ll}
\mathbf{G}^{1} & \mathbf{G}^{12}
\end{array}\right]\left\{\begin{array}{c}
\mathbf{P}^{1} \\
-\mathbf{P}^{21}
\end{array}\right\}+\left\{\mathrm{T}^{1}\right\}
$$


e a eq.(5.3):

$$
\left[\begin{array}{lll}
\mathbf{H}^{2} & \mathbf{H}^{21} & \mathbf{H}^{23}
\end{array}\right]\left\{\begin{array}{c}
\mathbf{U}^{2} \\
\mathbf{U}^{21} \\
\mathbf{U}^{32}
\end{array}\right\}=\left[\begin{array}{lll}
\mathbf{G}^{2} & \mathbf{G}^{21} & \mathbf{G}^{23}
\end{array}\right]\left[\begin{array}{c}
\mathbf{P}^{2} \\
\mathbf{P}^{21} \\
-\mathbf{P}^{32}
\end{array}\right\}+\left\{\mathbf{T}^{2}\right\}
$$

Os sistemas de equações de cada uma das três sub-regiões podem ser agrupados em um único que represente o domínio global da placa e depois de consideradas as condições de contorno da forma:

$$
\left[\begin{array}{ccccccc}
\mathbf{A}^{1} & \mathbf{0} & \mathbf{0} & \mathbf{H}^{12} & \mathbf{0} & \mathbf{G}^{12} & \mathbf{0} \\
\mathbf{0} & \mathbf{A}^{2} & \mathbf{0} & \mathbf{H}^{21} & \mathbf{H}^{23} & -\mathbf{G}^{21} & \mathbf{G}^{23} \\
\mathbf{0} & \mathbf{0} & \mathbf{A}^{3} & \mathbf{0} & \mathbf{H}^{32} & \mathbf{0} & -\mathbf{G}^{32}
\end{array}\right]\left\{\begin{array}{l}
\mathbf{X}^{1} \\
\mathbf{X}^{2} \\
\mathbf{X}^{3} \\
\mathbf{U}^{21} \\
\mathbf{U}^{32} \\
\mathbf{P}^{21} \\
\mathbf{P}^{32}
\end{array}\right\}=\left\{\begin{array}{l}
\mathbf{B}^{1} \\
\mathbf{B}^{2} \\
\mathbf{B}^{3}
\end{array}\right\}+\left\{\begin{array}{c}
\mathbf{T}^{1} \\
\mathbf{T}^{2} \\
\mathbf{T}^{3}
\end{array}\right\}
$$

onde $\mathbf{H}^{\mathrm{ij}}$ e $\mathbf{G}^{\mathrm{ij}}$ valem para $\mathbf{i} \neq \mathbf{j}$ e estão relacionadas a nós sobre interfaces cujos valores nodais são normalmente incógnitos, como visto anteriormente. Nesta equação, o termo $\mathbf{A}^{\mathrm{i}}$ representa os coeficientes de $\mathbf{H}^{\mathrm{i}}$ ou $\mathbf{G}^{\mathrm{i}}$ relativos a valores incógnitos de $\mathbf{U}^{\mathrm{i}}$ ou $\mathbf{P}^{\mathrm{i}}$, estes representados por $\mathbf{X}^{\mathrm{i}}$ na eq. 5.7. Por outro lado, os termos $\mathbf{B}^{\mathrm{i}}$ representam o produto dos coeficientes de $\mathbf{H}^{\mathrm{i}}$ ou $\mathbf{G}^{\mathrm{i}}$ por seus respectivos valores de contorno de, $\mathbf{U}^{\mathrm{i}}$ ou $\mathbf{P}^{\mathrm{i}}$. Note que, ambos os coeficientes $\mathbf{H}^{\mathrm{ij}}$ e $\mathbf{G}^{\mathrm{ij}}$ relacionados a interfaces são mantidos do lado direito da equação, mantendo seus sinais originais, exceto os coeficientes $\mathbf{G}^{\mathrm{ji}}$. Os coeficientes de uma sub-região estão sobre a mesma coluna que os correspondentes coeficientes das sub-regiões a ela adjacentes em cada interface. Note-se ainda que os sinais originais dos coeficientes $\mathbf{G}^{\mathrm{ji}}$, para $\mathbf{j}>\mathbf{i}$, são invertidos.

\footnotetext{
${ }^{1} \mathrm{O}$ sistema tem número de linhas suficiente para calcular uma das incógnitas, U ou P. A outra incógnita se obtém pela aplicação das condições de contorno, aspecto válido apenas para os nós fora das interfaces. Faltam, portanto, as variáveis que estão sobre as interfaces.
} 
Este exemplo é um caso simples em que cada interface conecta apenas duas sub-regiões, e ainda, as equações de equilíbrio e compatibilidade de deslocamentos, estabelecidas para os nós destas interfaces, não consideram influências externas. Portanto, os próximos passos serão reescrever a equação eq. 5.7 contando com estas possibilidades.

\section{3 - SUB-REGIÕES EM PLACAS DE KIRCHHOFF}

Usando o exemplo de duas placas, para o subdomínio $\boldsymbol{\Omega}^{1}$,

$\mathbf{H}_{\mathbf{u}}{ }^{1} \mathbf{u}^{1}+\mathbf{H}_{\theta}{ }^{1} \theta^{1}+\mathbf{H}_{\mathbf{u}}{ }^{12} \mathbf{u}^{12}+\mathbf{H}_{\theta}{ }^{12} \theta^{12}=\mathbf{G}_{\mathbf{V}}{ }^{1} \mathbf{V}^{1}+\mathbf{G}_{\mathbf{M}}{ }^{1} \mathbf{M}^{1}+\mathbf{G}_{\mathbf{V}}{ }^{12} \mathbf{V}^{12}+\mathbf{G}_{\mathbf{M}}{ }^{12} \mathbf{M}^{12}+\mathbf{T}^{1}$

e para o subdomínio $\Omega^{2}$

$$
\mathbf{H}_{\mathbf{u}}{ }^{2} \mathbf{u}^{2}+\mathbf{H}_{\theta}{ }^{2} \theta^{2}+\mathbf{H}_{\mathbf{u}}{ }^{21} \mathbf{u}^{21}+\mathbf{H}_{\theta}{ }^{21} \theta^{21}=\mathbf{G}_{\mathbf{V}}{ }^{2} \mathbf{V}^{2}+\mathbf{G}_{\mathbf{M}}{ }^{2} \mathbf{M}^{2}+\mathbf{G}_{\mathbf{V}}{ }^{21} \mathbf{V}^{21}+\mathbf{G}_{\mathbf{M}}{ }^{21} \mathbf{M}^{21}+\mathbf{T}^{2}
$$
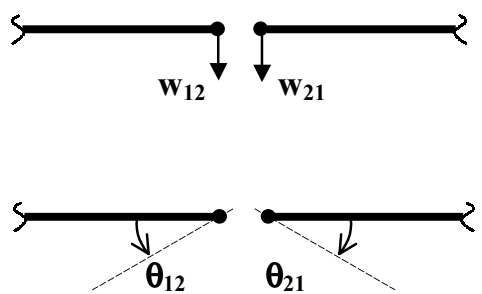
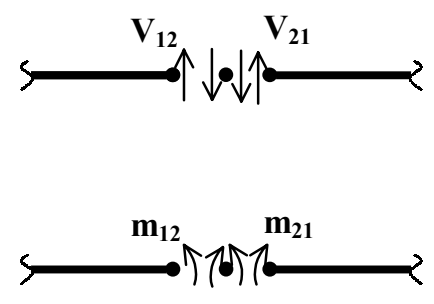

Fig. 5.2 - Variáveis associadas aos pontos do contorno das sub-regiões na região da interface -sentidos positivos-

Quando as variáveis envolvidas são relativas ao vetor $\overrightarrow{\mathbf{n}}$ normal ao contorno, as condições de equilíbrio e compatibilidade de deslocamentos serão, conforme a figura:

$$
\begin{array}{ll}
\mathbf{u}^{12}=\mathbf{u}^{21} & \mathbf{V}^{12}=-\mathbf{V}^{21} \\
\boldsymbol{\theta}^{12}=-\boldsymbol{\theta}^{21} & \mathbf{m}^{12}=\mathbf{m}^{21}
\end{array}
$$


Fazendo

$$
\begin{aligned}
& A^{1}=\mathbf{H}_{u}{ }^{1} \text { ou }-G_{v}{ }^{1} \text { ou } H_{\theta}{ }^{1} \text { ou }-G_{m}{ }^{1} \\
& \mathbf{X}^{1}=u^{1} \text { ou } V^{1} \text { ou } \theta^{1} \text { ou } \mathbf{m}^{1} \\
& \mathbf{B}^{1}=\mathbf{G}_{\mathbf{v}}{ }^{1} \mathbf{V}^{1} \text { ou }-\mathbf{H}_{\mathbf{u}}{ }^{1} \mathbf{u}^{1} \text { ou } \mathbf{G}_{\mathrm{m}}{ }^{1} \mathrm{~m}^{1} \text { ou }-\mathbf{H}_{\theta}{ }^{1} \boldsymbol{\theta}^{1}
\end{aligned}
$$

o que depende das condições de contorno, obtém-se

$$
\left.\left[\begin{array}{cccccc}
\mathbf{A}^{1} & \mathbf{0} & \mathbf{H}_{\mathrm{u}}{ }^{12} & \mathbf{H}_{\theta}{ }^{21} & \mathbf{G}^{12} & -\mathbf{G}^{12} \\
\mathbf{0} & \mathbf{A}^{2} & \mathbf{H}_{\mathrm{u}}{ }^{21}-\mathbf{H}_{\theta}{ }^{21} & -\mathbf{G}^{21} & -\mathbf{G}^{12}
\end{array}\right] \begin{array}{c}
\mathbf{X}^{1} \\
\mathbf{X}^{2} \\
\mathbf{u}^{12} \\
\theta^{12} \\
\mathbf{V}^{21} \\
\mathbf{m}^{12}
\end{array}\right\}=\left\{\begin{array}{l}
\mathbf{B}^{1} \\
\mathbf{B}^{2}
\end{array}\right\}+\left\{\begin{array}{l}
\mathbf{T}^{1} \\
\\
\mathbf{T}^{2}
\end{array}\right\}
$$

Outro aspecto a se cuidar é com relação às reações de canto na região da interface. A consideração deste esforço naquela região provoca perturbações nos resultados das variáveis calculadas nas proximidades do canto. Além disto, na maioria dos casos, o canto não existe efetivamente. Vários estudos sobre esta situação foram feitos (BETTAHAR \& WEARING(1993), VENTURINI (1988), PALERMO JR. (1989)) com a intenção de modelar o canto de interfaces adequadamente.

A exemplo da solução adotada por pesquisadores como, por exemplo, PALERMO JR.(1989), adotou-se, neste trabalho desprezar a parcela $\mathbf{w}_{\mathbf{c}}^{*} \mathbf{R}_{\mathbf{c}}$ da equação integral de deslocamentos. Como o mesmo não pode ser feito com o termo $\mathbf{R}_{\mathbf{c}}^{*} \mathbf{w}_{\mathbf{c}}$, este valor foi redistribuído entre os nós duplos da placa anterior (nant) e posterior (npos) ao canto em questão. Substitui-se, portanto, $\mathbf{w}_{\mathbf{c}}$ por: 


$$
\mathbf{w}_{\mathrm{c}}=\frac{\mathbf{w}_{(\text {nant })}+\mathbf{w}_{(\text {npos })}}{2}
$$

Uma conseqüência deste procedimento é a alteração no valor calculado de $\mathbf{V}_{\mathbf{n}}$ sobre os nós próximos ao canto (fig. 5.3) de $\mathbf{H}_{\mathrm{c}} / \mathbf{2}$.

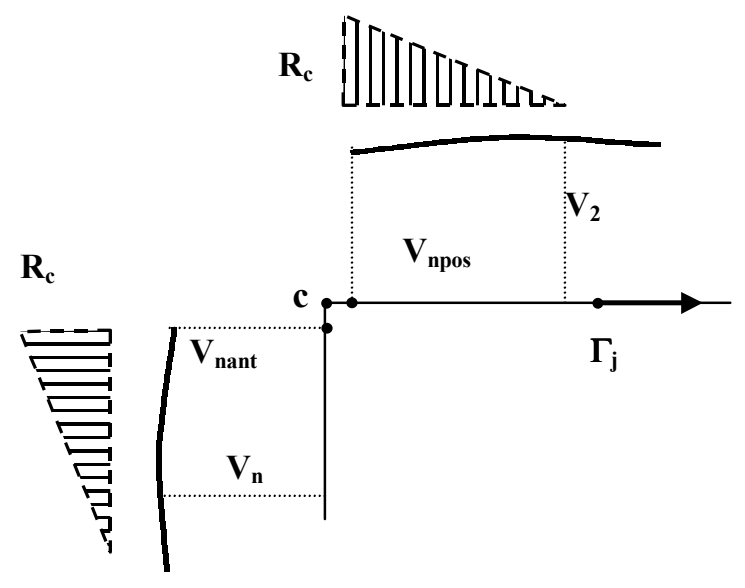

Fig. 5.3 - Redistribuição da reação do canto

\section{4 - SUB-REGIÕES ACOPLADAS NUMA INTERFACE COM INFLUÊNCIA EXTERNA}

Suponha-se que existem duas sub-regiões, $\boldsymbol{\Omega}^{1}$ e $\boldsymbol{\Omega}^{2}$ interconectadas ao longo da interface $\Gamma_{12}$, como mostra abaixo a fig.5.3:

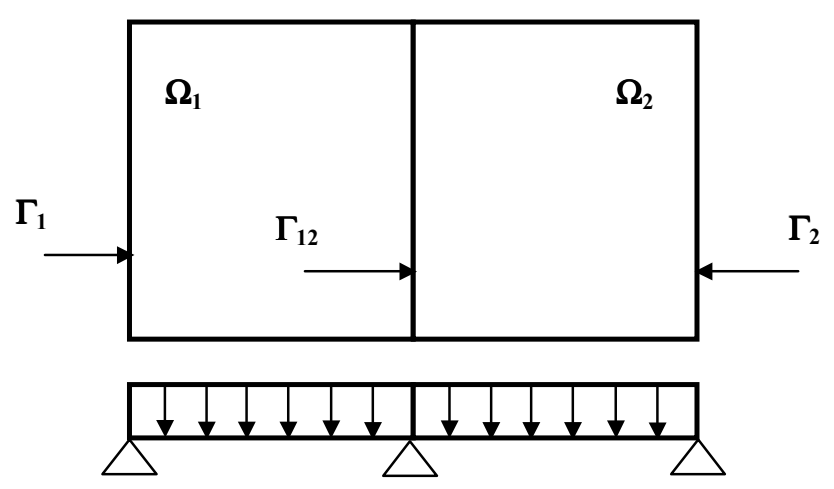


Fig. 5.4 - Interface entre sub-regiões apoiada

Considere-se agora, o caso em que as variáveis $\mathbf{P}$ e $\mathbf{U}$ em pontos da interface não são ambas desconhecidas, i.e., uma delas tem valor de contorno conhecido. Isto pode ocorrer, p.e., no caso de um apoio locado ao longo de uma interface ou num ponto da mesma (fig. 5.3). Ou ainda, se uma força externa é ali aplicada. No primeiro exemplo, surgirá uma reação de apoio na interface, $\mathbf{R}_{12}$, e serão conhecidos os deslocamentos $\mathbf{U}^{12}=\mathbf{U}^{21}$.

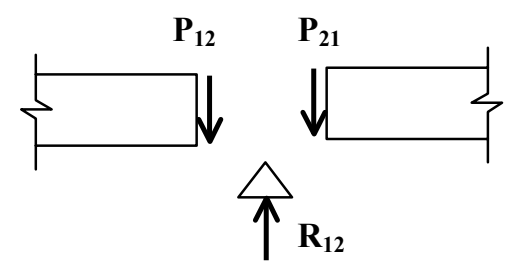

Fig. 5.5 - Esforços na região da interface $\Gamma_{12}$

Assim, têm-se dois grupos de esforços incógnitos na interface e apenas um de reações de apoio. Como existe um grupo de valores incógnitos a mais sobre o contorno da interface (comparando á análise de interfaces na seção anterior) resta então se calcular mais um conjunto de equações que permitam a resolução do problema. Torna-se necessário reescrever a equação equilíbrio:

$$
\mathbf{P}^{12}+\mathbf{P}^{21}+\mathbf{R}_{12}=0
$$

ou

$$
\mathbf{P}^{12}=-\left(\mathbf{P}^{21}+\mathbf{R}_{2}\right)
$$

Daí pode-se escrever para a sub-região 1:

$$
\mathbf{H}^{1} \mathbf{U}^{1}+\mathbf{H}^{12} \mathbf{U}^{21}=\mathbf{G}^{1} \mathbf{P}^{1}+\mathbf{G}^{12} \mathbf{P}^{12}+\mathbf{T}^{1}
$$

$\mathrm{ou}$

$$
\mathbf{H}^{1} \mathbf{U}^{1}+\mathbf{H}^{12} \mathbf{U}^{21}=\mathbf{G}^{1} \mathbf{P}^{1}-\mathbf{G}^{12} \mathbf{P}^{21}-\mathbf{G}^{12} \mathbf{R}_{12}+\mathbf{T}^{1}
$$


e para a sub-região 2:

$$
\mathbf{H}^{2} \mathbf{U}^{2}+\mathbf{H}^{21} \mathbf{U}^{21}=\mathbf{G}^{2} \mathbf{P}^{2}+\mathbf{G}^{21} \mathbf{P}^{21}+\mathbf{T}^{2}
$$

Para a sub-região 1:

$$
\left[\begin{array}{ll}
H^{1} & \mathbf{H}^{12}
\end{array}\right]\left\{\begin{array}{c}
\mathbf{U}^{1} \\
\mathbf{U}^{21}
\end{array}\right\}=\left[\begin{array}{lll}
\mathbf{G}^{1} & -\mathbf{G}^{12} & -\mathbf{G}^{12}
\end{array}\right]\left\{\begin{array}{l}
\mathbf{P}^{1} \\
\mathbf{P}^{21} \\
\mathrm{R}_{12}
\end{array}\right\}+\left\{\mathbf{T}^{1}\right\}
$$

E depois de consideradas as condições de contorno:

$$
\left[\begin{array}{lll}
A^{1} & G^{12} & G^{12}
\end{array}\right]\left\{\begin{array}{l}
X^{1} \\
P^{21} \\
R_{12}
\end{array}\right\}=\left\{B^{1}\right\}+\left\{T^{1}\right\}
$$

E, portanto, para a sub-região 2:

$$
\left[\begin{array}{ll}
\mathbf{A}^{2}-\mathbf{G}^{21}
\end{array}\right]\left\{\begin{array}{l}
\mathbf{X}^{2} \\
\mathbf{P}^{21}
\end{array}\right\}=\left\{\mathbf{B}^{2}\right\}+\left\{\mathbf{T}^{2}\right\}
$$

A forma final do sistema será agora:

$$
\left[\begin{array}{cccc}
A^{1} & 0 & G^{12} & G^{12} \\
0 & A^{2} & 0 & -G^{21}
\end{array}\right]\left\{\begin{array}{l}
X^{1} \\
X^{2} \\
R_{12} \\
P^{21}
\end{array}\right\}=\left\{\begin{array}{l}
B^{1} \\
B^{2}
\end{array}\right\}+\left\{\begin{array}{l}
T^{1} \\
T^{2}
\end{array}\right\}
$$


Este caso pode ocorrer em qualquer ponto de interface ao qual se pode aplicar as condições de contorno. No caso das placas de Kirchhoff isto pode acontecer facilmente nos cantos, ou em lajes cogumelo.

Note-se que apenas os coeficientes $\mathbf{G}^{\mathrm{ij}}$ para os pontos de interface são mantidos e repetidos do lado esquerdo da equação e apenas para uma das sub-regiões que se interfaceiam. Os respectivos coeficientes $\mathbf{H}^{\mathrm{ij}}$ foram multiplicados pelos seus correspondentes valores de $\mathbf{U}^{\mathrm{ij}}$ e resultados adicionados em $\mathbf{A}^{\mathrm{i}}$. Para a outra subregião (subdomínio $\boldsymbol{\Omega}^{2}$ neste exemplo) mantêm-se os $\mathbf{G}^{\mathrm{ij}}$ apenas uma vez e as colunas relativas às reações $\mathbf{R}_{12}$ de apoio são preenchidas por zeros. Como no caso analisado na seção anterior, os sinais originais dos coeficientes $\mathbf{G}^{\mathrm{ji}}$, para $\mathbf{j}>\mathbf{i}$, são invertidos. Aqui também os coeficientes de uma sub-região estão sobre a mesma coluna que os correspondentes coeficientes das sub-regiões a ela adjacentes em cada interface.

\section{5 - MAIS DE DUAS SUB-REGIÕES ACOPLADAS NUMA MESMA INTERFACE}

Considere-se agora o caso em que mais de duas sub-regiões estejam conectadas por uma mesma interface, como nos exemplos da fig.(5.5). $\mathrm{Na}$ fig.(5.5-a), p.e., existem três sub-regiões, $\boldsymbol{\Omega}^{1}, \boldsymbol{\Omega}^{2}$ e $\boldsymbol{\Omega}^{3}$ e quatro interfaces, $\Gamma^{12}, \Gamma^{13}, \Gamma^{23}$ (estas três excluindo o ponto $\mathbf{k}$ ) e o ponto $\mathbf{k}$ (um ponto que interconecta as três sub-regiões). $\mathrm{Na}$ fig.(5.5-c),considerando a viga $\mathbf{\Omega}^{3}$ como uma sub-região que compatibiliza os deslocamentos de ambas as sub-regiões $\boldsymbol{\Omega}^{1}$ e $\boldsymbol{\Omega}^{2}$, existe agora uma única interface $\Gamma^{123}$. Na fig.(5.5-b), a interface entre as três sub-regiões é o ponto $\mathbf{k}$. 

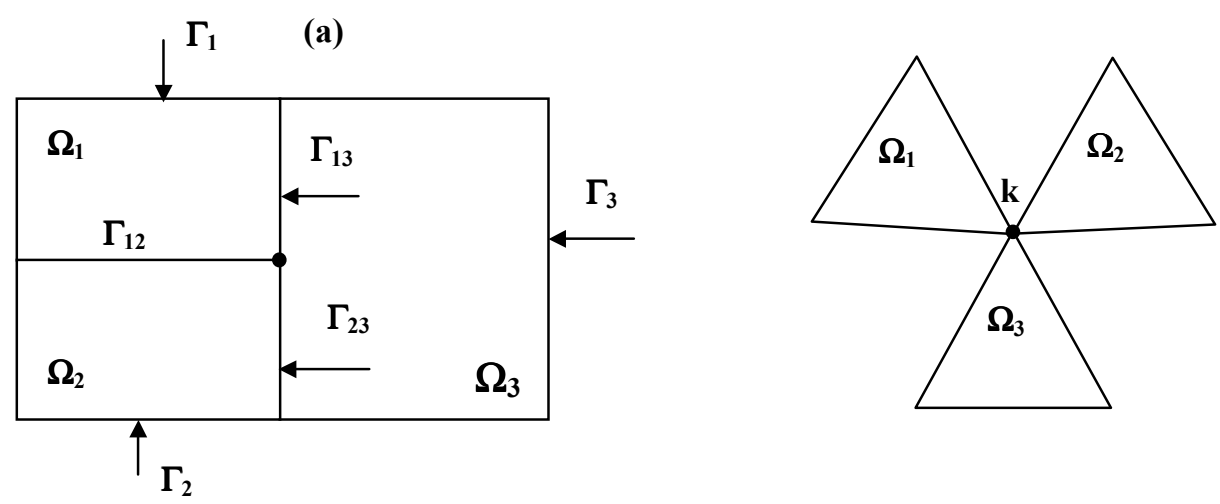

(b)

Fig. 5.6a e b-Mais de uma sub-região sobre por interface

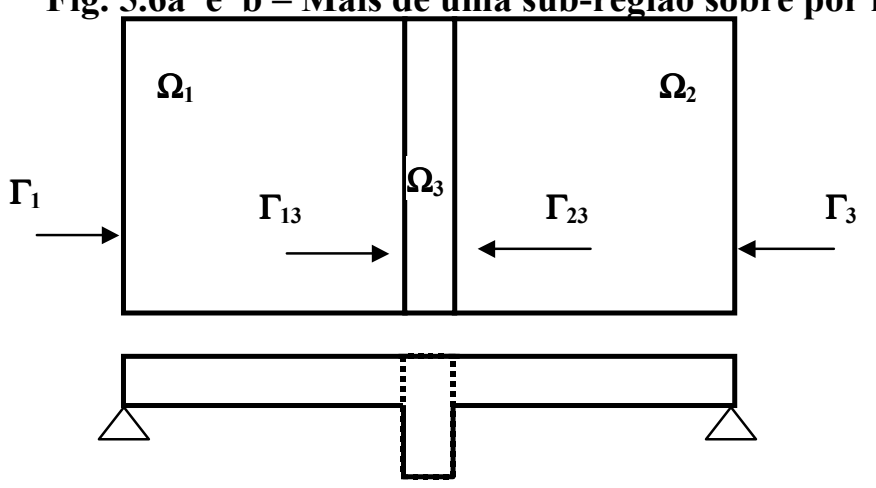

(c)

Fig. 5.6c - Mais de uma sub-região sobre por interface

A forma matricial geral do sistema que define o exemplo na fig.(5.5-b) é:

$$
\mathbf{H U}=\mathbf{G P}+\mathbf{T}
$$

que particionado para considerar os coeficientes relativos aos pontos externos e sobre a interface (neste caso o nó $\mathbf{k}$ apenas) pode ser escrito para cada sub-região:

$$
\left[\begin{array}{ll}
\mathbf{H}^{\mathrm{i}} & \mathbf{H}_{k}^{\mathrm{i}}
\end{array}\right]\left\{\begin{array}{c}
\mathbf{U}^{\mathrm{i}} \\
\mathbf{U}_{k}^{\mathrm{i}}
\end{array}\right\}=\left[\begin{array}{ll}
\mathbf{G}^{\mathrm{i}} & \mathbf{G}_{\mathrm{k}}^{\mathrm{i}}
\end{array}\right]\left\{\begin{array}{l}
\mathbf{P}^{\mathrm{i}} \\
P_{k}^{i}
\end{array}\right\} \quad \mathbf{i}=\mathbf{1 , 3}
$$

As condições de equilíbrio e compatibilidade de deslocamentos fornecerão as três equações que faltam: 


$$
\begin{array}{ll}
\mathbf{U}^{1}{ }_{\mathrm{k}}=\mathbf{U}_{\mathrm{k}}{ }_{\mathrm{k}}=\mathbf{U}_{\mathrm{k}}^{3} & \left(=\mathbf{U}_{\mathrm{k}}\right) \\
\mathbf{P}_{\mathrm{k}}^{1}+\mathbf{P}_{\mathrm{k}}{ }_{\mathrm{k}}+\mathbf{P}_{\mathrm{k}}^{3}=\mathbf{0} & \text { ou } \quad \mathbf{P}_{\mathrm{k}}^{1}=-\left(\mathbf{P}_{\mathrm{k}}^{2}+\mathbf{P}^{3}{ }_{\mathrm{k}}\right)
\end{array}
$$

Seguindo o mesmo procedimento adotado até aqui, a forma matricial para este sistema, considerando-se agora todo o domínio agrupado e juntamente com as condições de contorno é:

$$
\left[\begin{array}{cccccc}
\mathbf{A}_{1} & \mathbf{0} & \mathbf{0} & \mathbf{H}_{1}^{\mathbf{k}} & \mathbf{G}_{1}^{\mathbf{k}} & \mathbf{G}_{1}^{\mathbf{k}} \\
\mathbf{0} & \mathbf{A}_{2} & \mathbf{0} & \mathbf{H}_{2}^{\mathbf{k}} & -\mathbf{G}_{2}^{\mathbf{k}} & \mathbf{0} \\
\mathbf{0} & \mathbf{0} & \mathbf{A}_{3} & \mathbf{H}_{3}^{\mathbf{k}} & \mathbf{0} & -\mathbf{G}_{3}^{\mathbf{k}}
\end{array}\right]\left\{\begin{array}{l}
\mathbf{X}_{1} \\
\mathbf{X}_{2} \\
\mathbf{X}_{3} \\
\mathbf{U}^{\mathbf{k}} \\
\mathbf{P}_{2}^{\mathbf{k}} \\
\mathbf{P}_{3}^{\mathbf{k}}
\end{array}\right\}=\left\{\begin{array}{l}
\mathbf{B}_{1} \\
\mathbf{B}_{2} \\
\mathbf{B}_{3}
\end{array}\right\}+\left\{\begin{array}{l}
\mathbf{T}_{1} \\
\mathbf{T}_{2} \\
\mathbf{T}_{3}
\end{array}\right\}
$$

Resolvido o sistema, resta obter-se os esforços $\mathbf{P}_{\mathbf{1}}{ }^{\mathbf{k}}$, que são calculados na eq.(5.12).

Para efeito de programação, para cada interface Int com um total de número de sub-regiões nela concorrentes NSPI(Int), observa-se a partir do exemplo apresentado o seguinte:

- como cada ponto da interface Int terá um valor de deslocamento $\mathbf{U}^{\mathbf{k}}$, aloca-se apenas $\mathbf{2}$-Nnint(Int) colunas na matriz do sistema final para armazenamento dos coeficientes da matriz $\mathbf{H}$, sendo Nnint(Int) o número de nós da interface Int. Isto é, todos os coeficientes $\mathbf{H}$ relativos aos nós da interface Int estarão sobre a mesma coluna.

- como resultado da imposição do equilíbrio da forma apresentada neste trabalho pela eq.(5.6), o resultado da solução do sistema fornecerá os esforços nos nós de interface relativos às sub-regiões $\boldsymbol{\Omega}_{\mathbf{2}}$ a $\boldsymbol{\Omega}_{\mathrm{NSPI}(\mathrm{Int}) \cdot}$. Os esforços para a $\boldsymbol{\Omega}_{1}$ vêm do equilíbrio, que de uma forma geral se escreve:

$$
\mathbf{P}_{1}^{(\text {Int })}=-\sum_{i=2}^{\text {NSPI (Int) }} \mathbf{P}_{i}^{(\text {Int })}
$$


- o número de colunas da matriz $\mathbf{G}$ a se anexar à matriz final, portanto, será 2·Nnint(Int)·[NSPI(Int)-1]. Nas linhas relativas à sub-região $\boldsymbol{\Omega}_{\mathbf{1}}$, os $\mathbf{G}_{\mathbf{1}}{ }^{\text {(Int) }}$ serão armazenados com seus sinais originais e repetidamente tantas vezes quanto for o número de sub-regiões menos 1 (NSPI(Int)-1). Nas linhas das sub-regiões restantes, nas colunas relativas aos seus respectivos valores de esforços, serão armazenados apenas uma vez os coeficientes $\mathbf{G}_{\mathbf{i}}{ }^{(\mathbf{I n t})}$, porém com sinais trocados. As demais colunas serão preenchidas por zeros.

Desta forma, pode-se escrever para o exemplo da fig. 5.5-a:

$$
\left[\begin{array}{cccccccccccc}
\mathbf{A}_{1} & \mathbf{0} & \mathbf{0} & \mathbf{H}_{12} & \mathbf{H}_{13} & \mathbf{0} & \mathbf{H}_{1}^{\mathrm{k}} & \mathbf{G}_{12} & \mathbf{G}_{13} & \mathbf{0} & \mathbf{G}_{1}^{\mathbf{k}} & \mathbf{G}_{1}^{\mathbf{k}} \\
\mathbf{0} & \mathbf{A}_{2} & \mathbf{0} & \mathbf{H}_{21} & \mathbf{0} & \mathbf{H}_{23} & \mathbf{H}_{2}^{\mathrm{k}} & -\mathbf{G}_{21} & \mathbf{0} & \mathbf{G}_{23} & -\mathbf{G}_{2}^{\mathbf{k}} & \mathbf{0} \\
\mathbf{0} & \mathbf{0} & \mathbf{A}_{3} & \mathbf{0} & \mathbf{H}_{31} & \mathbf{H}_{32} & \mathbf{H}_{3}^{\mathrm{k}} & \mathbf{0} & -\mathbf{G}_{31} & -\mathbf{G}_{32} & \mathbf{0} & -\mathbf{G}_{3}^{\mathbf{k}}
\end{array}\right]\left\{\begin{array}{l}
\mathbf{X}_{1} \\
\mathbf{X}_{2} \\
\mathbf{X}_{3} \\
\mathbf{U}_{21} \\
\mathbf{U}_{31} \\
\mathbf{U}_{32} \\
\mathbf{U}^{\mathbf{k}} \\
\mathbf{P}_{21} \\
\mathbf{P}_{31} \\
\mathbf{P}_{32} \\
\mathbf{P}_{2}^{\mathbf{k}} \\
\mathbf{P}_{3}^{\mathbf{k}}
\end{array}\right\}=\left\{\begin{array}{l}
\mathbf{B}_{1} \\
\mathbf{B}_{2} \\
\mathbf{B}_{3}
\end{array}\right\}+\left\{\begin{array}{c}
\mathbf{T}_{1} \\
\mathbf{T}_{2} \\
\mathbf{T}_{3}
\end{array}\right\}
$$

- Nenhuma modificação é sofrida pelos termos independentes $\left\{\mathbf{B}_{\mathbf{i}}\right\}$ e $\left\{\mathbf{T}_{\mathbf{i}}\right\}$

Esta é a forma geral a formulação de uma placa dividida em sub-regiões. Um cuidado, porém deve ser tomado quando da elaboração do algoritmo: ao se trocar o sinal original do coeficiente $\mathbf{G}^{\mathbf{j i}}$, para $\mathbf{j}>\mathbf{i}$, deve-se observar a convenção de sinais adotada para os esforços no programa básico para a análise de flexão em placas. No programa elaborado por CHUEIRI(1994), por exemplo, os sentidos dos esforços e são tais que, para 2 sub-regiões adjacentes, o equilíbrio nos pontos da interface se estabelece na forma (vide fig. 5.2):

$$
\mathrm{V}_{\mathrm{I}}+\mathrm{V}_{\mathrm{II}}=\mathbf{0} \quad \Rightarrow \quad \mathrm{V}_{\mathrm{I}}=-\mathrm{V}_{\mathrm{II}}
$$

mas, 
$\mathbf{M}_{\mathrm{I}}-\mathrm{M}_{\mathrm{II}}=\mathbf{0} \quad \Rightarrow \quad \mathbf{M}_{\mathrm{I}}=\mathrm{M}_{\mathrm{II}}$

ou seja, a mudança do sinal dependerá de que tipo de esforço o coeficiente $\mathbf{G}^{\mathbf{j i}},(\mathbf{j}>\mathbf{i})$ está multiplicando.

\section{5 - UM PROCEDIMENTO ALTERNATIVO PARA A CONSIDERAÇÃO DE SUBDIVISÃO DO DOMÍNIO}

VENTURINI \& PAIVA (1987) apresentaram um procedimento alternativo para se considerar o domínio em estudo composto por sub-regiões. A proposta apresentada pelos autores foi a de se evitar a subdivisão física do domínio em regiões de mesma rigidez. A idéia básica era se considerar uma proporcionalidade entre as soluções fundamentais definidas para cada sub-região, uma vez que cada uma delas teria espessuras (t) distintas. Nesta seção, este método será apresentado, apesar de não ter sido o método usado nesta tese e sim o da subdivisão física do domínio em regiões.

Utilizando-se o exemplo analisado por VENTURINI \& PAIVA (1987), os termos relativos à solução fundamental para a sub-região $\boldsymbol{\Omega}_{1}$ usará o símbolo “*” e para a sub-região $\boldsymbol{\Omega}_{2}$, “**'. Para cada região $\mathbf{m}$ :

$$
\begin{aligned}
& w *(q, p)=\frac{1}{8 \pi D_{1}} r^{2}\left(\ln r-\frac{1}{2}\right) \\
& w * *(q, p)=\frac{1}{8 \pi D_{2}} r^{2}\left(\ln r-\frac{1}{2}\right)
\end{aligned}
$$

onde

$$
D_{m}=\frac{E t_{m}^{3}}{12\left(1-v^{2}\right)}
$$

é a rigidez à flexão da placa. 
VENTURINI \& PAIVA (1987) utilizaram o símbolo ‘*'para os esforços de modo geral, pois as variáveis fundamentais independem de $\mathbf{D}$.

Pode-se escrever a solução fundamental de qualquer placa como função de $\mathbf{w} *(\mathbf{q}, \mathbf{p})$ na forma:

$$
\mathbf{D}_{2} \mathbf{w} *(\mathbf{q}, \mathbf{p})=\mathbf{D}_{1} \mathbf{w} *(\mathbf{q}, \mathbf{p})
$$

ou melhor:

$$
\mathbf{w} *(\mathbf{q}, \mathbf{p})=\left(\mathbf{D}_{1} / \mathbf{D}_{2}\right) \mathbf{w} *(\mathbf{q}, \mathbf{p})
$$

De uma forma geral, portanto, pode-se dizer que qualquer sub-região $\boldsymbol{\Omega}_{\mathrm{k}} \mathrm{de}$ rigidez $\mathbf{D}_{\mathbf{k}}$ terá a sua solução fundamental $\left(\mathbf{w}^{\mathbf{k} *}\right)$ escrita em função de $\left(\mathbf{w}^{*}\right)$ proveniente da sub-região de rigidez $\mathbf{D}$, de forma que:

$$
w^{k} *(q, p)=\left(D / D_{k}\right) w *(q, p)
$$

Do teorema de Betti pode-se escrever para cada sub-região $\mathbf{k}$ a relação:

$$
\int_{\Omega_{\mathrm{k}}} m_{\mathrm{ij}} * w,_{\mathrm{ij}} \mathrm{d} \Omega_{\mathrm{k}}=\int_{\Omega_{\mathrm{k}}} m_{\mathrm{ij}} \mathbf{w},{ }_{\mathrm{ij}}{ }^{k} \mathrm{~d} \Omega_{\mathrm{k}}
$$

Computando-se a influência de todos os subdomínios, considerando-se $\mathbf{w}^{*}$ a solução fundamental relativa à sub-região de rigidez $\mathbf{D}$, pode-se escrever:

$$
\int_{\Omega} m_{\mathrm{ij}} * \mathbf{w}, \mathrm{ij} \Omega=\int_{\Omega} \mathbf{m}_{\mathrm{ij}} \mathrm{w}_{\mathrm{ij}}{ }^{*} \mathrm{~d} \Omega
$$

Então, deve-se suprimir o efeito de $\mathbf{w}^{*}$ sobre a sub-região $\boldsymbol{\Omega}_{\mathrm{k}}$ e finalmente somar a contribuição de suas próprias soluções fundamentais $\mathbf{w}^{* *}$ ao domínio global: 


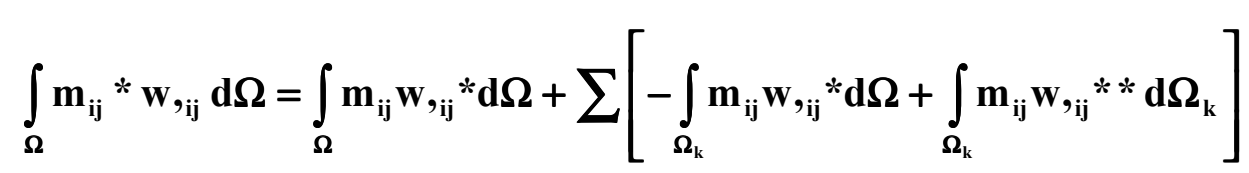

Considerando-se a relação em eq. 5.20:

$$
\int_{\Omega} m_{\mathrm{ij}} * \mathbf{w},{ }_{\mathrm{ij}} \mathrm{d} \Omega=\int_{\Omega} \mathbf{m}_{\mathrm{ij}} \mathbf{w},{ }_{\mathrm{ij}} * \mathrm{~d} \Omega+\sum \frac{D_{\mathrm{k}}}{D}\left[-\int_{\Omega} m_{\mathrm{ij}} \mathbf{w},{ }_{\mathrm{ij}} * * d \Omega+\int_{\Omega_{\mathrm{k}}} \mathbf{m}_{\mathrm{ij}} \mathbf{w},,_{\mathrm{ij}} * * d \Omega_{\mathrm{k}}\right]
$$

e finalmente aplicando-se a igualdade eq. 5.21, obtém-se:

$$
\int_{\Omega} m_{\mathrm{ij}} * w_{\mathrm{ij}} d \Omega=\int_{\Omega} m_{\mathrm{ij}} \mathbf{w}_{\mathrm{ij}} * \mathrm{~d} \Omega-\sum \frac{D_{\mathrm{k}}-\mathrm{D}}{D}\left[\int_{\Omega_{\mathrm{k}}} \mathrm{m}_{\mathrm{ij}} * \mathbf{w},_{\mathrm{ij}} \mathrm{d} \Omega_{\mathrm{k}}\right]
$$

Usando-se, portanto o procedimento para se obter a equação integral para o cálculo dos deslocamentos verticais $\mathbf{w}$ para placas isoladas sobre a eq. 5.25 acima, chega-se finalmente a:

$$
\begin{aligned}
& \mathbf{w}(\mathbf{q})+\int_{\Gamma}\left(\mathbf{V}_{\mathrm{n}} *(\mathbf{q}, \mathbf{P}) \mathbf{w}(\mathbf{P})-\mathbf{m}_{\mathrm{n}} *(\mathbf{q}, \mathbf{P}) \mathbf{w},{ }_{\mathrm{n}}(\mathbf{P})\right) \mathrm{d} \Gamma(\mathbf{P})+\sum_{\mathrm{i}=1}^{\mathrm{N}_{\mathrm{c}}} \mathbf{R}_{\mathrm{ci}} *(\mathbf{q}, \mathbf{P}) \mathbf{w}_{\mathrm{ci}}(\mathbf{P})= \\
& \int_{\Gamma}\left(V_{n}(q, P) w *(P)-m_{n}(q, P) w,,_{n}^{*}(P)\right) d \Gamma(P)+ \\
& \sum_{i=1}^{N_{c}} R_{c i}(q, P) w_{c i} *(P)+\int_{\Omega g} g(p) w *(q, p) d \Omega_{g}(p)- \\
& \sum \frac{D_{k}-D}{D}\left[\int_{\Gamma}\left(V_{n} *(q, P) w(P)-m_{n} *(q, P) w, ~(P)\right) d \Gamma(P)+\sum_{i=1}^{N_{c}} R_{c i} *(q, P) w_{c i}(P)\right]
\end{aligned}
$$

Os autores confrontaram com sucesso resultados desta formulação para exemplos numéricos com resultados obtidos através de um programa de elementos finitos para análise de placas.

\section{6 - EXEMPLOS}


Nesta seção, apresentam-se exemplos da aplicação da técnica das sub-regiões, no intuito de demonstrar a eficiência da formulação apresentada neste capítulo.

\subsection{1 - EXEMPLOS I, II e III}

VENTURINI \& PAIVA (1987) utilizaram os três primeiros dos exemplos aqui mostrados, nos quais a mesma placa quadrada é analisada sob carregamento uniformemente distribuído (q) e para três condições de apoio diferentes. Esta placa é dividida em duas sub-regiões retangulares de mesma largura e propriedades físicas e geométricas (especialmente espessuras) diferentes, conforme se vê nos diagramas (figs. 5.7a a 5.7c).

No primeiro exemplo (figs. 5.7a), a placa quadrada simplesmente apoiada nos bordos, foi dividida em duas sub-regiões de rigidezes a flexão $\mathbf{D}_{\mathbf{1}}$ e $\mathbf{D}_{\mathbf{2}}$. A mesma placa é analisada no exemplo II (figs. 5.7b), porém com os bordos externos engastados. No terceiro exemplo (figs. 5.7c), dois bordos opostos da placa estão simplesmente apoiados e os outros dois em balanço.

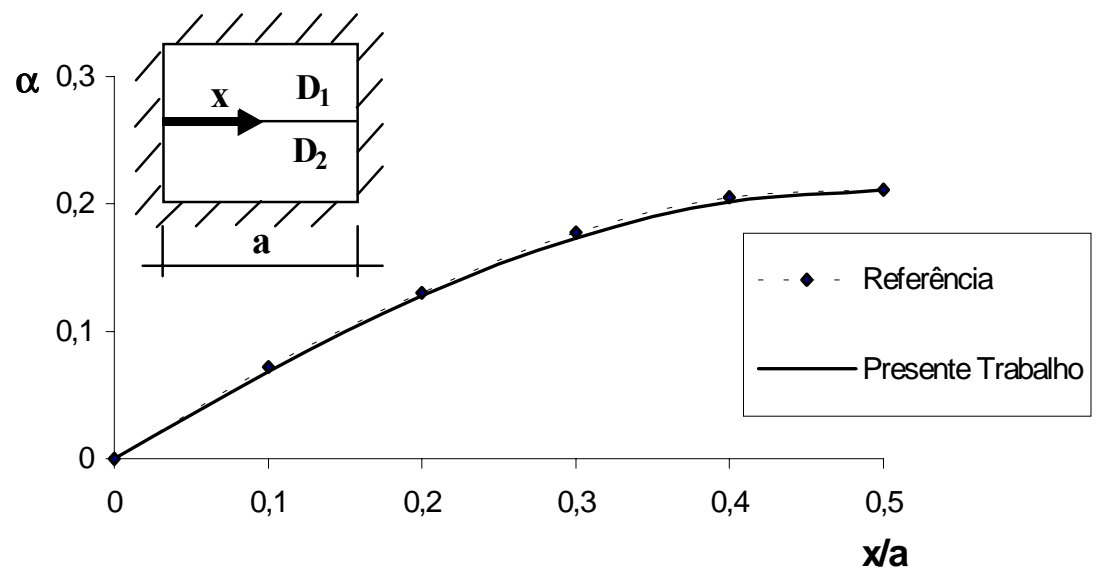

Fig. 5.7a - EXEMPLO I: Placa quadrada simplesmente apoiada. ( $v=0)$

Fator multiplicativo $\alpha=\left(w \cdot 10^{2} \cdot D_{1}\right) /\left(q^{2} a^{2}\right)$ 
Nos diagramas das fig. 5.7a, b e c, pode-se observar a grande proximidade entre os resultados obtidos para o presente trabalho (que utiliza sub-rotina de acoplamento entre sub-regiões) e os calculados através do método apresentado por VENTURINI \& PAIVA (1987) (item 5.5).

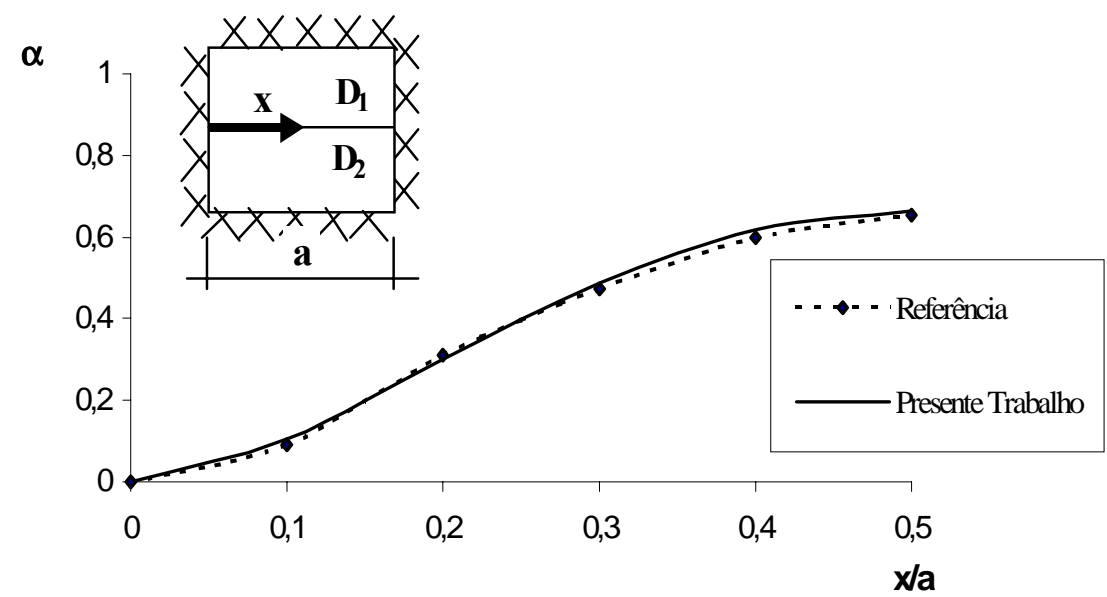

Fig 5.7b- EXEMPLO II: Placa quadrada totalmente engastada $(v=0)$ Fator multiplicativo $\quad \alpha=\left(w \cdot 10^{3} \cdot D_{1}\right) /\left(q^{2}{ }^{2}\right)$ 


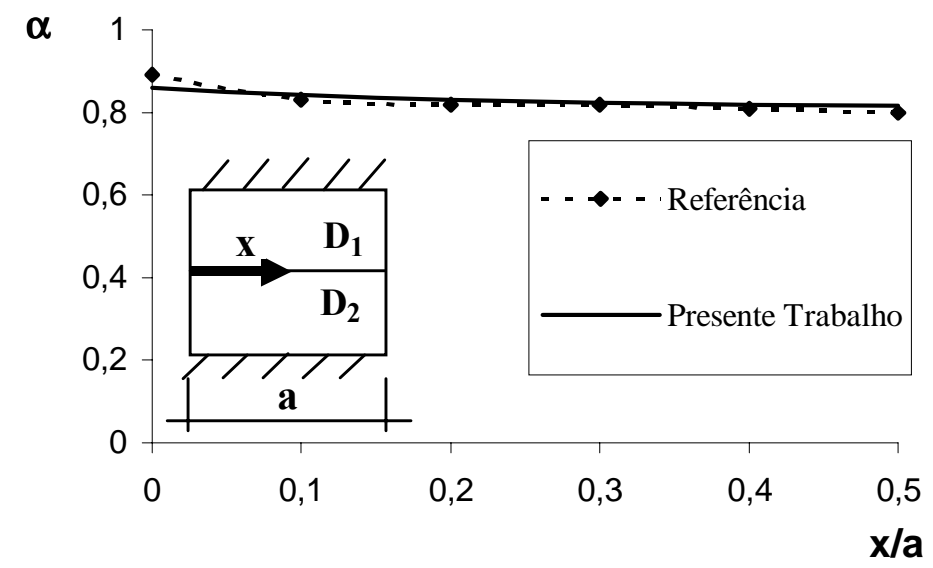

Fig. 5.7c- EXEMPLO I: Placa quadrada com dois bordos opostos livres e dois $\operatorname{apoiados}(v=0,3)$

$$
\text { Fator multiplicativo } \quad \alpha=\left(w \cdot 10^{2} \cdot D_{1}\right) /\left(\mathbf{q a}^{2}\right)
$$

\subsection{3 - EXEMPLO IV}

Neste exemplo, deseja-se demonstrar porque se deve fazer a subdivisão do domínio de uma placa de forma criteriosa e apenas quando realmente existir a descontinuidade nas regiões das interfaces definidas. Para tanto, utiliza-se uma placa quadrada simples, retirada de TIMOSHENKO (1959), de espessura constante no seu domínio (fig. 5.8). O carregamento sobre ela é uniformemente distribuído e a placa possui dois dos seus bordos opostos simplesmente apoiados e os outros dois engastados. 


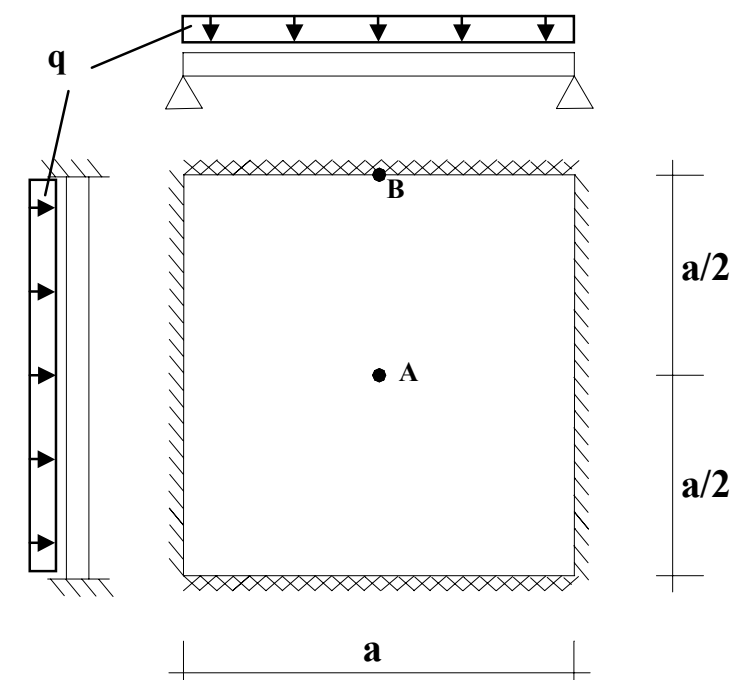

Fig. 5.8-Placa quadrada com dois bordos opostos engastados e dois apoiados $\mathbf{v}=\mathbf{0 , 3}$ (Timoshenko (1959))

Os resultados de deslocamentos verticais e momentos em $\mathbf{x}$ e em $\mathbf{y}$ nos pontos A e B, obtidos nesta tese e os calculados por TIMOSHENKO (1959) para a placa sem subdivisões, são mostrados na tab. 5.1. As figuras nas primeiras colunas desta tabela indicam a direção da interface e a posição em que o ponto $\mathbf{A}$ foi considerado.

Tab. 5.1-Placa quadrada com dois bordos opostos livres e dois apoiados (Timoshenko (1959) - resultados

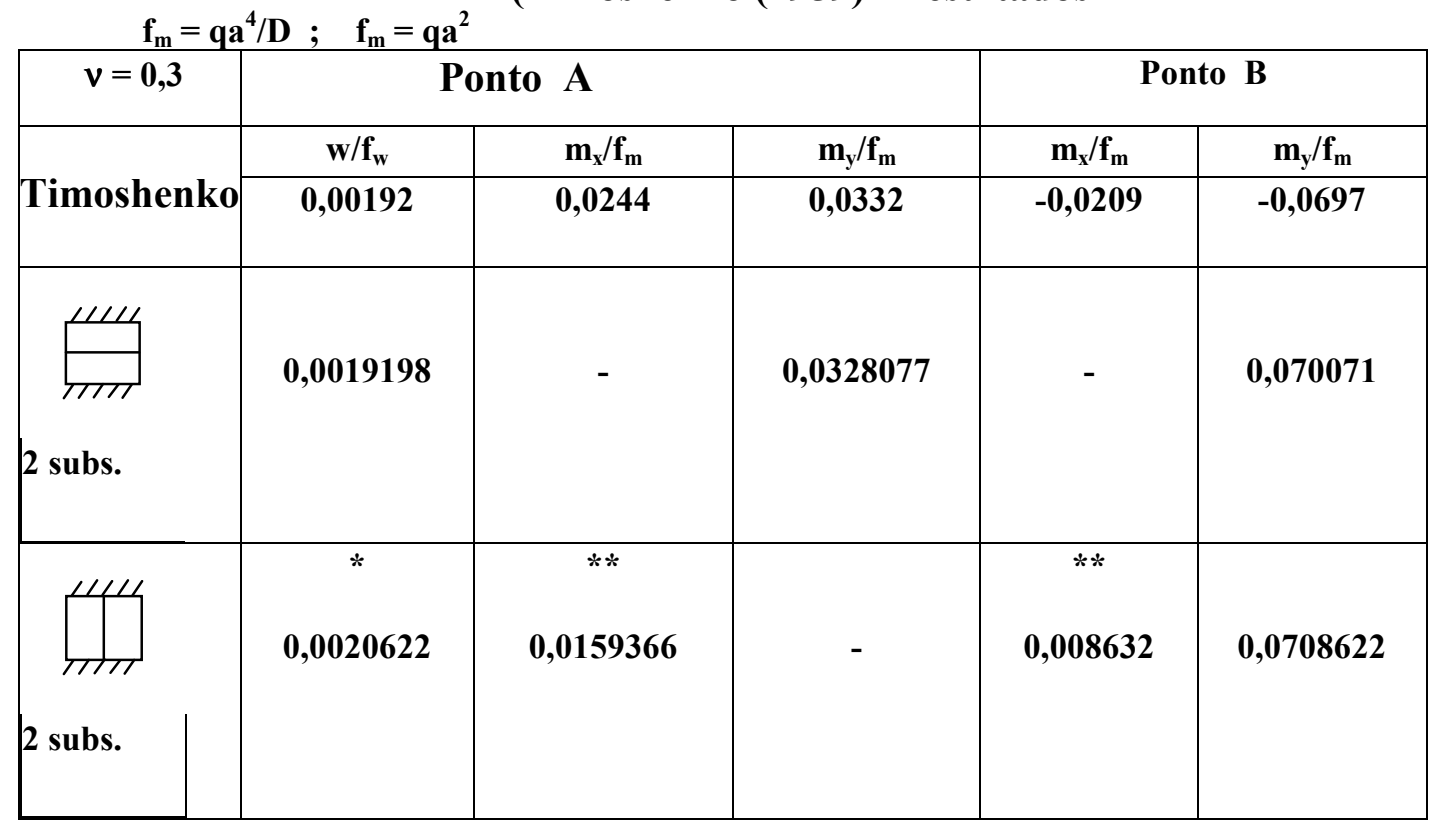




\begin{tabular}{|c|c|c|c|c|c|}
\hline 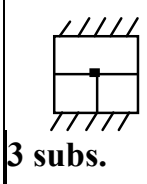 & 0,0019576 & - & 0,031784 & - & 0,06951 \\
\hline 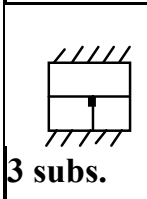 & $\begin{array}{c}* \\
0,0022179\end{array}$ & 0,02595567 & - & $\begin{array}{c}* * \\
0,003822\end{array}$ & $\begin{array}{c}* \\
0,0591\end{array}$ \\
\hline $\begin{array}{l}\qquad 114 \\
y=1 \\
4 \text { subs. }\end{array}$ & 0,001989 & - & $\begin{array}{c}* \\
0,0293933\end{array}$ & $\begin{array}{c}* * \\
0,0038\end{array}$ & $\begin{array}{c}* \\
0,059329\end{array}$ \\
\hline $\begin{array}{l}\qquad 4 \\
4 \pi \\
4 \text { subs. }\end{array}$ & $\begin{array}{c}* * \\
0,0021469\end{array}$ & 0,0263675 & - & $\begin{array}{c}* * \\
0,0038\end{array}$ & 0,059329 \\
\hline
\end{tabular}

Comparando-se estes valores, percebem-se as discrepâncias que podem ocorrer nos resultados se a divisão do domínio é feita sem critério e desnecessariamente, como alertou VENTURINI(1987) (os asteriscos ' *' indicam os valores que mais se distanciam dos resultados teóricos de TIMOSHENKO (1959); dois asteriscos '**, indicam os valores muito diferentes dos calculados pela teoria)

Como se viu em 5.5, a formulação elaborada por VENTURINI \& PAIVA (1987) para se evitar a subdivisão física do domínio em regiões inclui menos aproximações na equação dos deslocamentos na região das interfaces. A comparação entre os resultados obtidos por este método ou pela formulação exata (como se apresenta em TIMOSHENKO (1959), p.e.) demonstra tanto que a rotina desenvolvida nesta tese conduz ao cálculo de resultados com boa aproximação. Além disso, qualquer que seja a técnica de subdivisão do domínio da placa adotada, há que se usar do bom senso para ao definir as posições e direções das interfaces. 


\section{CAPÍTULO 6 - COMBINAÇÃO MEC/MEF}

\section{1- INTRODUÇÃO}

Diversas técnicas e sugestões de pesquisadores foram utilizadas nos vários tipos de problemas estruturais para combinar o MEC e o MEF, como HARTLEY(1996), PAIVA(1996), BREBBIA \& GEOUGIOU (1979), VENTURINI \& PAIVA(1987), CODA(1993), KOMATSU et al. (1997), BEER(1986), TANAKA \& BERCIN (1997), MESSAFER \& COATES (1989). Estes trabalhos, dentre vários outros, contribuíram para evidenciar a possibilidade da utilização do MEC combinado com outros métodos numéricos. Pode-se, portanto combinar regiões de quaisquer propriedades mecânicas, de comportamentos estruturais de diferentes naturezas, equacionadas por métodos numéricos que melhor descrevam seus comportamentos.

De acordo com VENTURINI(1988), atribui-se aos trabalhos de ZIENKIEWICZ et al. (1977), SHAW \& FALBY (1977), OSIAS et al. (1977) os primeiros trabalhos propostos de combinação entre o MEC e o MEF. 
TANAKA \& BERCIN (1997) trabalharam sobre o pavimento e se preocuparam em incluir o efeito da excentricidade do eixo dos enrijecedores de placas, de seções quaisquer, na formulação do comportamento do conjunto MEC/MEF. O efeito dos enrijecedores é transferido, como na maioria dos trabalhos sobre o assunto, para formulação da placa.

PALERMO JR. (1989) estudou peças (barras) de seções abertas delgadas, analisadas como placas acopladas no espaço. Os efeitos de placa e de chapa foram associados, porém, independentemente entre si, numa mesma barra, e associados sempre que esta fosse composta por placas não-coplanares.

Conforme exposto anteriormente, neste trabalho será feita a ligação entre os elementos estruturais planos do pavimento modelados pelo MEC e os lineares modelados pelo MEF. As equações algébricas, como se sabe, foram elaboradas separadamente. Para efetuar a junção será utilizada a técnica das sub-regiões, cujas vantagens já foram expostas no capítulo 5.

Na formulação utilizada para a análise de flexão de placas consideram-se, para cada um de seus pontos discretos do contorno, duas componentes de esforços $\left(\mathbf{V}_{\mathbf{n}} \mathrm{e}\right.$ $\mathbf{m}_{\mathbf{n}}$ ) e duas de deslocamentos ( $\mathbf{w}$ e $\boldsymbol{\theta}_{\mathbf{n}}$ ), sendo as variáveis com sub-índice $\underline{\mathbf{n}}$ são definidas em relação ao versor normal ao seu contorno. No presente estudo, estas serão as variáveis consideradas no estabelecimento do equilíbrio e da compatibilidade de deslocamentos, respectivamente, na região da ligação com as barras, procedendo as devidas correções, como se verá adiante. Nestas regiões, portanto, haverá o equilíbrio entre os esforços cortantes e dos momentos fletor e torsor da barra. A compatibilidade, da mesma forma, será verificada entre os deslocamentos correspondentes a estes esforços.

Existem trabalhos, como PAIVA(1996), PAIVA(1987), NG, CHEUNG \& $\mathbf{X U}(\mathbf{1 9 9 0})$, por exemplo, em que se considera o equilíbrio apenas entre as forças verticais internas existentes entre placas e barras. Outros, como 
OLIVEIRA NETO(1998) e novamente PAIVA(1987) apresentam formulação com a consideração da rotação tangencial ao contorno da placa, permitindo a compatibilização inclusive nesta direção.

No presente trabalho, escolheu-se adotar um sistema global de coordenadas para o conjunto placa/barras para propiciar o acoplamento entre regiões de EC e EF. As coordenadas locais das barras e placas (estas últimas definidas em relação ao versor normal ao seu contorno) serão, portanto, transformadas para o citado sistema global de coordenadas.

Diante das características de cada método, portanto, foram feitas algumas aproximações para que este acoplamento seja possível, conforme as considerações expostas nos itens que seguem. Inicialmente e para facilitar o entendimento, irá se considerar a união entre os efeitos de flexão de placas e de barras, sem a consideração do efeito de membrana. A partir do item 6.7 então, as variáveis relativas ao EPT serão introduzidas no sistema e novas e devidas aproximações serão feitas.

\section{2 - COORDENADAS GLOBAIS DOS ELEMENTOS ESTRUTURAIS}

Para elaboração da rotina de acoplamento, será adotado o seguinte sistema global de referência (fig. 6.1), coincidente com o do EF, definido no cap. 4.

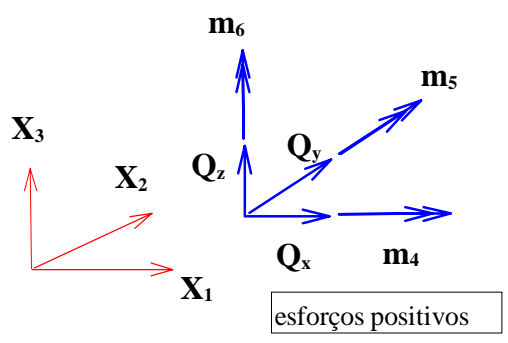

Fig. 6.1 - Coordenadas globais dos elementos estruturais 
Na rotina de cálculo dos elementos de contorno, as componentes $\mathbf{V}_{\mathbf{n}}, \mathbf{M}_{\mathbf{n}}$ e $\boldsymbol{\theta}_{\mathbf{n}}$ foram definidas em relação ao versor normal ao contorno, conforme a fig. 6.2:

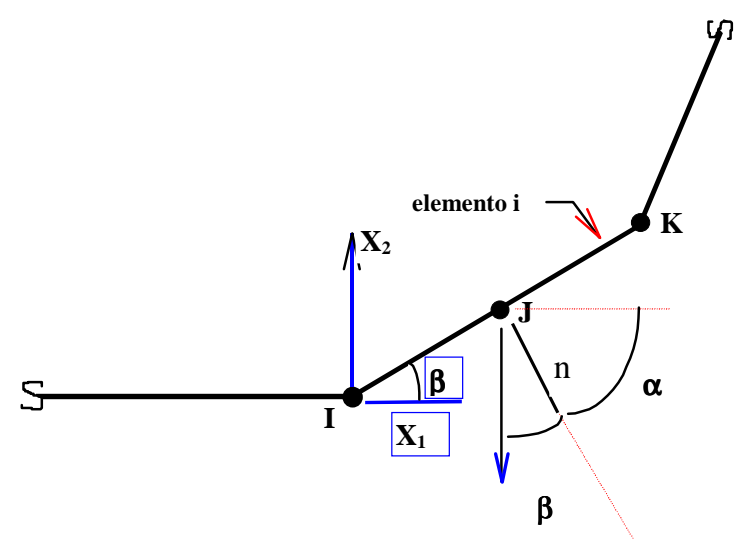

Fig. 6.2 - Sistema local de coordenadas dos elementos de placa

\subsection{1 - ESFORÇOS EM UM PONTO DA PLACA}

Pode-se obter as componentes dos esforços da placa do sistema local de coordenadas, considerando-se o sistema global da forma apresentada na fig. 6.3.

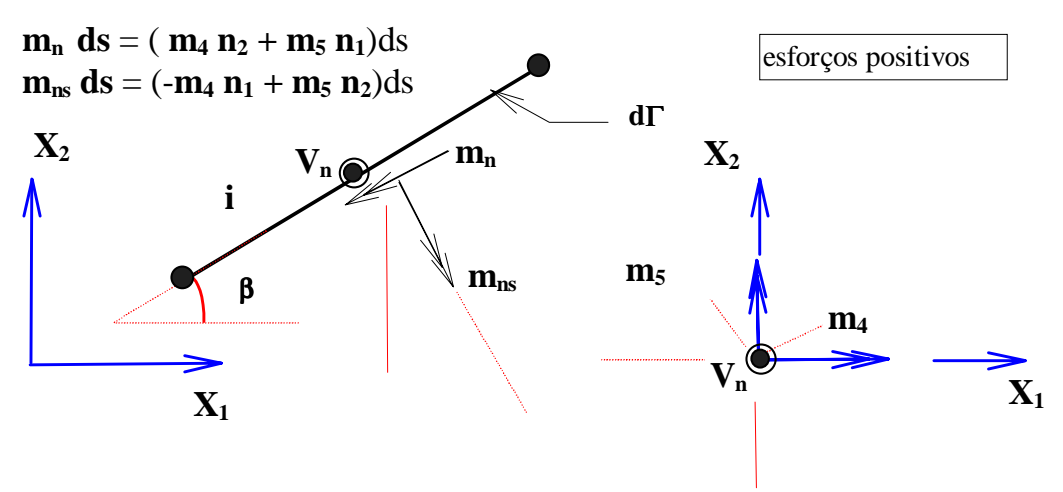

Fig. 6.3 - Transformação das componentes de esforços da placa

Para que os esforços da placa sejam escritos considerando-se o sistema global adotado, deve-se proceder a transformação, de uma forma geral e matricial para cada nó i, na forma: 


$$
\left\{\begin{array}{l}
\mathbf{V}_{n} \\
\mathbf{m}_{\mathbf{n}} \\
\mathbf{m}_{\mathrm{ns}}
\end{array}\right\}=\underbrace{\left[\begin{array}{ccc}
1 & 0 & 0 \\
\mathbf{0} & \mathbf{n}_{2} & \mathbf{n}_{1} \\
\mathbf{0} & -\mathbf{n}_{1} & \mathbf{n}_{2}
\end{array}\right]}_{\mathbf{T}_{\mathrm{e}}}\left\{\begin{array}{l}
\mathbf{Q}_{3} \\
\mathbf{m}_{4} \\
\mathbf{m}_{5}
\end{array}\right\}
$$

ou

$$
\begin{aligned}
\mathbf{P}_{\mathbf{n}}{ }^{\mathrm{i}} & =\mathbf{T}_{\mathrm{e}} \mathbf{P}_{\mathrm{g}}^{\mathrm{i}} \\
& =\mathbf{T}_{\mathbf{p}} \mathbf{P}_{\mathrm{g}}^{\mathbf{i}}
\end{aligned}
$$

onde os sub-índices $\mathbf{n}$ e $\mathbf{g}$ referem-se, respectivamente, aos sistemas local normal e ao global; $\mathbf{T}_{\mathbf{e}}$ é a matriz de transformação das variáveis entre os dois sistemas e $\mathbf{P}$ referese aos esforços.

\subsection{2 - DESLOCAMENTOS EM UM PONTO DA PLACA}

No sistema de referências local do elemento da placa, para o qual foi desenvolvido o algoritmo, sabe-se que:

$$
\theta_{\mathbf{n}}=d \mathbf{d w} / \mathrm{dn}
$$

Analogamente ao que foi feito para os esforços, pode-se escrever a transformação das componentes de deslocamentos do sistema local para o global de coordenadas, conforme a fig.6.4:

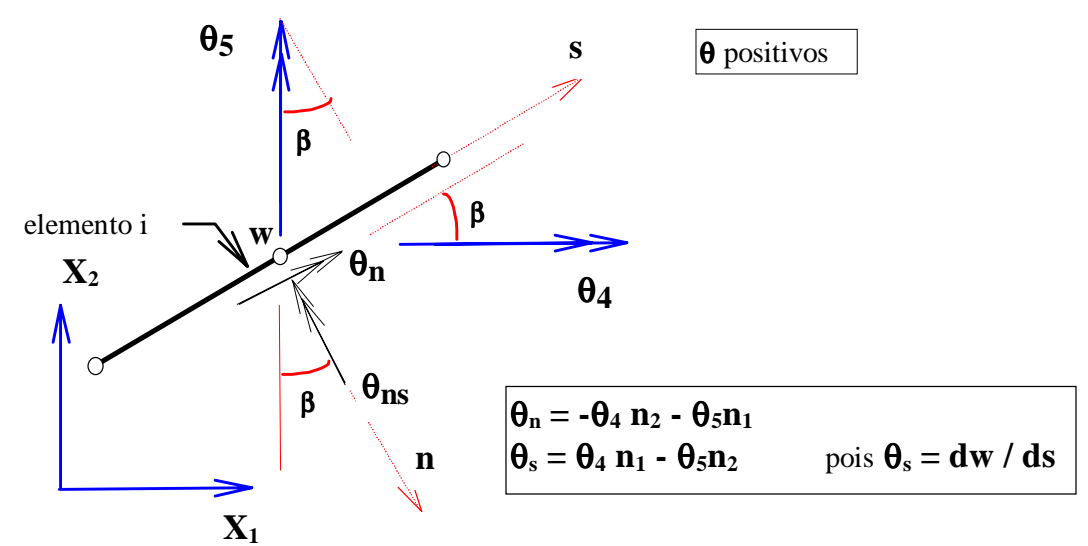

Fig. 6.4 - Transformação das componentes de deslocamento da placa 
Generalizando-se para cada nó i:

$$
\left\{\begin{array}{l}
w \\
\theta_{n} \\
\theta_{s}
\end{array}\right\}=\underbrace{\left[\begin{array}{ccc}
-1 & 0 & 0 \\
0 & -n_{2} & -n_{1} \\
0 & n_{1} & -n_{2}
\end{array}\right]}_{-T_{e}}\left\{\begin{array}{l}
w_{3} \\
\theta_{4} \\
\theta_{5}
\end{array}\right\}
$$

$\mathrm{Ou}$

$$
\begin{aligned}
\mathbf{U}_{\mathbf{n}}{ }^{i} & =-\mathbf{T}_{\mathbf{e}} \mathbf{U}_{\mathbf{g}}{ }^{\mathbf{i}} \\
& =\mathbf{T}_{\mathbf{U}} \mathbf{U}_{\mathbf{g}}{ }^{\mathbf{i}}
\end{aligned}
$$

o índice $\mathbf{U}$ refere-se aos deslocamentos.

\subsection{3 - TRANSFORMAÇÃO ENTRE COORDENADAS LOCAIS E GLOBAIS PARA N $\mathrm{N}_{n}$ PONTOS DA PLACA}

O vetor de esforços e deslocamentos de toda a placa de $\mathbf{N}_{n}$ pontos será:

$$
\begin{aligned}
& P_{\mathrm{n}}=T \mathbf{P}_{\mathrm{G}} \\
& \mathrm{U}_{\mathrm{n}}=-T \mathrm{U}_{\mathrm{G}}
\end{aligned}
$$

sendo, para $\mathbf{i}=\mathbf{1 , N}$ :

$$
\begin{aligned}
& P_{n}=\left\{P_{n}^{i}\right\}=\left\{\begin{array}{llllllllllll}
V_{n}^{1} & m_{n}^{1} & m_{n s}^{1} & \ldots & V_{n}^{i} & m_{n}^{i} & m_{n s}^{i} & \ldots & V_{n}^{N} & m_{n}^{N} & m_{n s}^{N}
\end{array}\right\}^{T} \\
& U_{n}=\left\{\begin{array}{lllllllllll}
U_{n}^{i}
\end{array}\right\}=\left\{\begin{array}{lllllllllll}
w^{1} & \theta_{n}^{1} & \theta_{n s}^{1} & \ldots & w^{i} & \theta_{n}^{i} & \theta_{n s}^{i} & \ldots & w^{N} & \theta_{n}^{N} & \theta_{n s}^{N}
\end{array}\right\}^{T} \\
& P_{G}=\left\{P_{g}^{i}\right\}=\left\{\begin{array}{llllllllllll}
Q_{3}^{1} & m_{4}^{1} & m_{5}^{1} & \ldots & Q_{3}^{i} & m_{4}^{i} & m_{5}^{i} & \ldots & Q_{3}^{N} & m_{4}^{N} & m_{5}^{N}
\end{array}\right\}^{T} \\
& \left.U_{G}=\left\{U_{g}^{i}\right\}=\begin{array}{lllllllllll}
w_{3}^{1} & \theta_{4}^{1} & \theta_{5}^{1} & \ldots & w_{3}^{i} & \theta_{4}^{i} & \theta_{5}^{i} & \ldots & w_{3}^{N} & \theta_{4}^{N} & \theta_{5}^{N}
\end{array}\right\}^{T}
\end{aligned}
$$


e

$$
\mathbf{T}=\left[\begin{array}{ccccc}
\mathbf{T}_{\mathrm{e}}^{\mathbf{1}} & \cdots & \mathbf{0} & \cdots & \mathbf{0} \\
\vdots & \ddots & \vdots & & \vdots \\
\mathbf{0} & \cdots & \mathbf{T}_{\mathrm{e}}^{\mathrm{i}} & \cdots & \mathbf{0} \\
\tilde{\vdots} & & \vdots & \ddots & \vdots \\
\mathbf{0} & \cdots & \mathbf{0} & \cdots & \mathbf{T}_{\mathrm{e}}^{\mathrm{N}}
\end{array}\right]_{3 \mathrm{~N} \times 3 \mathbf{N}}
$$

onde $\mathbf{T}_{\mathbf{e}} \mathbf{i}$ é a já vista matriz $\mathbf{T}_{\mathbf{e}}$ para cada nó $\mathbf{i}$. Observe-se que, apesar de serem apenas $\mathbf{w}, \boldsymbol{\theta}_{\mathrm{n}}, \mathbf{V}_{\mathrm{n}}$ e $\mathbf{m}_{\mathrm{n}}$ as variáveis do problema de flexão de placas, a dimensão da matriz $\mathbf{T}$ é exibida como $\mathbf{3} \mathbf{N}_{\mathrm{n}} \mathbf{3} \mathbf{3} \mathbf{N}_{\mathrm{n}}$. As matrizes $\mathbf{H}$ e $\mathbf{G}$, portanto, deverão ser expandidas também para $\mathbf{3} \mathbf{N}_{\mathrm{n}}$ linhas por $\mathbf{3} \mathbf{N}_{\mathrm{n}}$ colunas, ao invés de $\mathbf{2} \mathbf{N}_{\mathrm{n}} \mathrm{x} \mathbf{2} \mathbf{N}_{\mathrm{n}}$, para possibilitar a combinação com o sistema de barras. Estas linhas e colunas extras serão preenchidas por zeros. Como este procedimento só se realizará quando o acoplamento for feito entre sub-regiões de MEC com MEF, a singularidade do sistema final total será evitada pelas linhas equacionadas pelo MEF.

Daí, levando-se em conta as eq. 6.3, a eq. 2.62, relativa a coordenadas locais da placa, fica:

$$
\mathbf{H}_{\mathrm{G}} \mathbf{U}_{\mathrm{G}}=\mathbf{G}_{\mathrm{G}} \mathbf{P}_{\mathrm{G}}+\mathbf{T}
$$

onde $\mathbf{U}_{\mathrm{G}}$ e $\mathbf{P}_{\mathrm{G}}$ vêm das eq. 6.5, $\mathbf{H}_{\mathrm{G}}=\mathbf{- H} \cdot \mathbf{T}$ e $\mathbf{G}_{\mathrm{G}}=\mathbf{G} \cdot \mathbf{T}$.

\section{4 - COORDENADAS DOS ELEMENTOS DE BARRAS}

Conforme já visto, num elemento finito de barra, as coordenadas locais são indicadas abaixo, juntamente com os valores nodais de deslocamentos e esforços: 


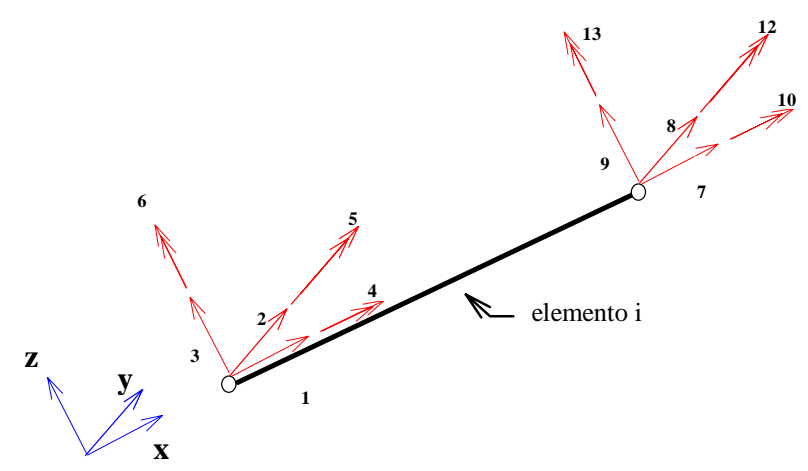

Fig. 6.5 - Coordenadas locais do elemento de barra

O algoritmo desenvolvido para descrever o comportamento estrutural das barras através do método dos elementos finitos, fornece as matrizes de rigidez $\underset{\sim}{\mathbf{K}}$ e de transformação do vetor de forças $\underset{\sim}{\mathbf{C}}$ (ver cap. 4) relacionadas ao sistema global de coordenadas. Este sistema é coincidente com o sistema aqui adotado como o global de referência para a ligação entre barras e placas.

\section{5 - CONDIÇÕES DE CONTORNO DA COMBINAÇÃO}

No presente trabalho, as condições de contorno das barras são consideradas em relação às coordenadas globais. No domínio das placas, porém, as condições de contorno são originalmente aplicadas em relação ao sistema local relativo à normal ao contorno, para efeito de utilização da rotina aqui apresentada. Deve-se, portanto, proceder a transformação das equações de condições de contorno, também, quando do acoplamento entre placas e barras.

Como se sabe, as condições de contorno de cada sub-região, bem como o equilíbrio de forças e a compatibilidade de deslocamentos entre qualquer par de subregiões sobre uma interface comum, são usadas para auxiliar na resolução do sistema de equações gerado. Serão abordados, a seguir, alguns aspectos particulares para efeito de utilização das condições de contorno com este fim, quando há combinação entre os sistemas gerados pelo MEC e pelo MEF. 


\subsection{1 - CONDIÇÕES DE CONTORNO NO MEF}

\subsubsection{1 - NÓS NÃO-LIGADOS}

Analisa-se agora o sistema de equações final, resultante da combinação dos sistemas formulados pelo MEC e MEF, separadamente, com relação aos nós não pertencentes a interfaces. A maneira com que se consideram as condições de contorno para a região do MEC, nos pontos externos a interfaces, permanece a mesma usada para a placa isolada. Pode-se, inclusive, fazê-lo durante a montagem das equações da sub-região, antes da combinação com o sistema de barras. O mesmo, porém, não pode ser dito a respeito da região de $\mathbf{E F}$, cuja equação de equilíbrio aqui se transcreve:

$$
\underset{\sim}{\mathbf{U}}=\underset{\mathbf{C}}{\mathbf{P}}+\underset{\sim}{\mathbf{E}}
$$

A forma convencional com que se consideram as condições de contorno na análise matricial de estrutura, porém, poderá implicar na colocação de valor nulo na diagonal principal do sistema de equações misto, isto é, o sistema formado por subregiões modeladas pelo MEC e pelo MEF, conforme a organização adotada neste trabalho. Não se pode, muito menos, trocar colunas entre $\underset{\sim}{\mathbf{K}}$ e $\underset{\sim}{\mathbf{C}}$, pois os valores de $\mathbf{P}$ são conhecidos inclusive para os pontos fora das regiões de interface. Por esta razão, um artifício será utilizado e se reescreverá a equação matricial eq. 6.8 da forma:

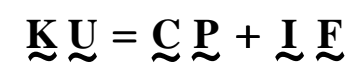

e a troca de colunas (relativas aos graus de liberdades restritos) será feita, portanto, entre $\underset{\sim}{\mathbf{K}}$ e a identidade $\underset{\mathbf{I}}{\mathbf{I}}$. Para um exemplo de uma barras discretizada por 4 nós, esta operação na região de $\mathbf{E F}$ resulta em: 


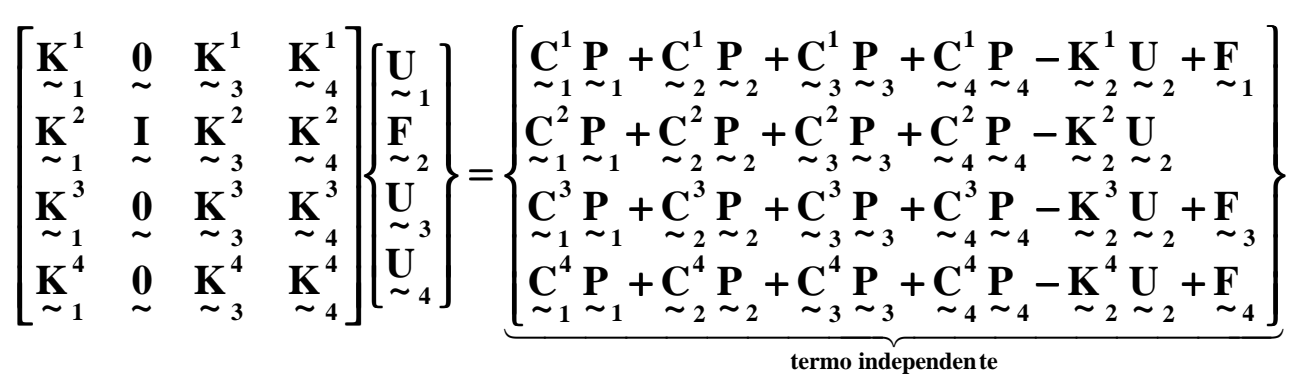

As incógnitas nestes pontos serão agora as forças e reações $\underset{\sim}{\mathbf{F}}$. Evita-se, desta forma, a introdução de uma linha de zeros na posição da variável em questão.

\subsubsection{2 - NÓS DE INTERFACE SEM INFLUÊNCIA EXTERNA}

Estudou-se no cap. 5 a formulação para o caso geral da união de duas subregiões. Para o caso específico da combinação de sistemas do MEC e do MEF, segue-se o mesmo esquema, lembrando-se apenas que ambos sistemas estarão transformados para o sistema global de coordenadas no momento da junção. As equações de equilíbrio e compatibilidade na região da interface serão:

$$
\begin{aligned}
& \underset{\sim}{\mathbf{P}_{\mathrm{F}}^{\mathrm{I}}}=-\underline{\sim}_{\underline{B}}^{\mathrm{I}} \\
& \underset{\sim}{\mathbf{U}_{\mathrm{F}}^{\mathrm{I}}}={\underset{\sim}{\mathrm{U}}}_{\mathbf{I}}^{\mathrm{I}}
\end{aligned}
$$

resultando no sistema final, após a consideração das condições de contorno de ambos os sub-domínios da forma:

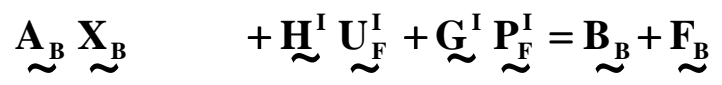

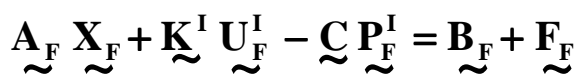


onde o sub-índice $\underline{\mathbf{F}}$ se refere aos nós da região de MEF ("finite") internos vinculados e $\underline{\mathbf{B}}$ aos nós da região de MEC (“boundary”). I está relacionado aos nós de interfaces.

\subsubsection{3 - NÓS DE INTERFACE COM INFLUÊNCIA EXTERNA}

No cap. 5 elaborou-se a formulação para o caso geral da união de duas subregiões onde são conhecidas as condições de contorno na região da interface. Aqui nesta seção, será realçada apenas a forma matricial para a união entre regiões de MEC e MEF para esta mesma situação. Após a devida transformação para o sistema global de coordenadas, as equações de equilíbrio e compatibilidade na região da interface serão:

$$
\begin{aligned}
& {\underset{\sim}{\mathrm{F}}}^{\mathrm{I}}=-\underset{\sim}{\mathbf{P}_{\mathbf{B}}^{\mathrm{I}}}-\underset{\sim}{\mathbf{R}^{\mathrm{I}}} \\
& \underset{\sim}{\mathbf{U}_{\mathbf{F}}^{\mathrm{I}}}=\underset{\sim}{\mathbf{U}_{\mathbf{B}}^{I}}
\end{aligned}
$$

resultando no sistema final:

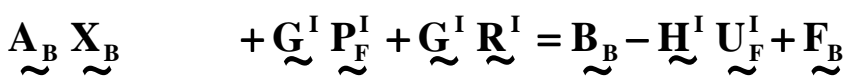

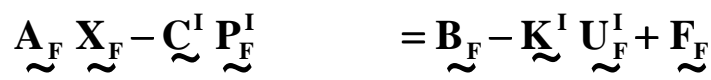

Esta é uma forma de se evitar o cálculo de integrais singulares que surgem quando a determinação das reações $\mathbf{R}^{\mathbf{I}}$ é feita através da vinculação de pontos do domínio da placa. Quando se vincula nós no domínio, a restrição dos deslocamentos provoca o surgimento de reações neles, introduzindo, desta forma, novas incógnitas ao problema. Tradicionalmente, novas equações são escritas para os deslocamentos dos citados nós de domínio vinculados, como se viu no cap. 2. 
Uma outra vantagem do procedimento acima, que utiliza a técnica das subregiões, sobre o tradicional, é que as rigidezes dos elementos de barras não precisam ser transferidas para a equação das sub-regiões de placas.
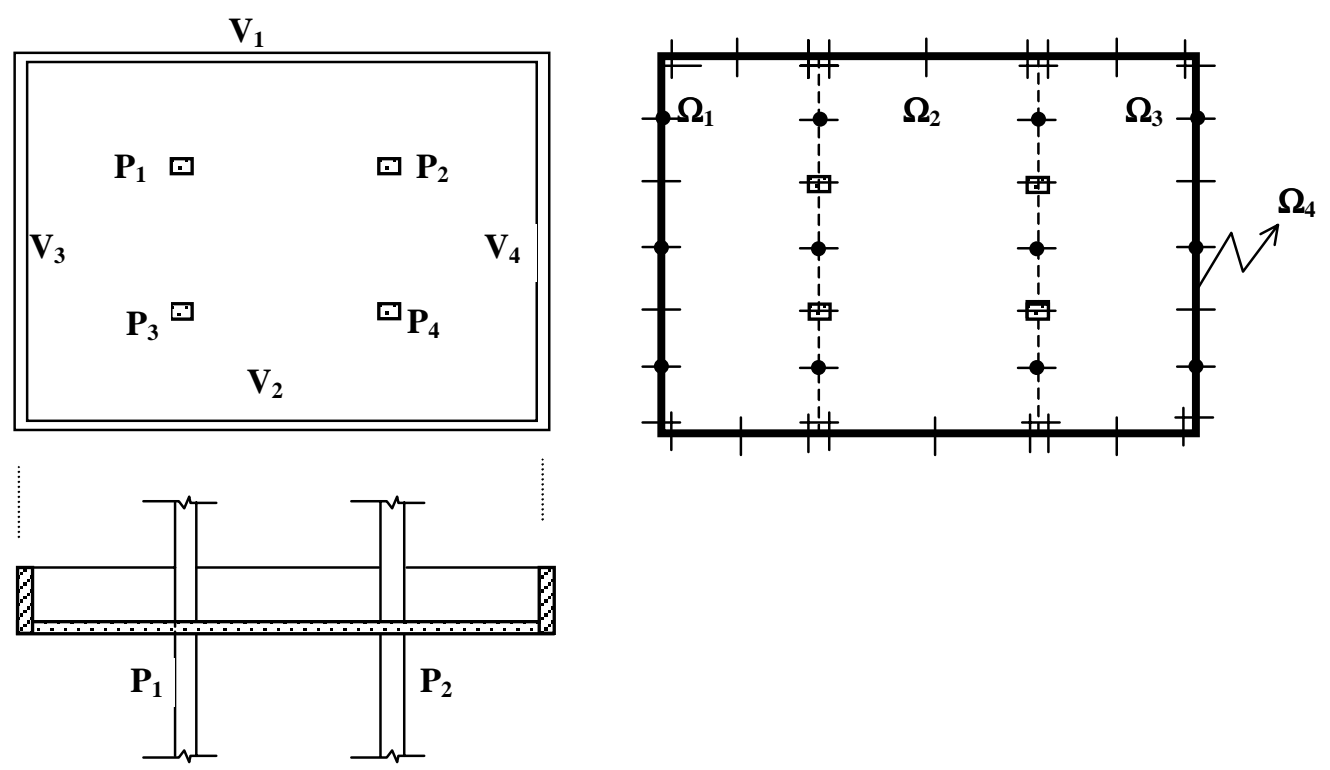

Fig. 6.6 - Sub-divisão de laje cogumelo para o cálculo da reações nos pilares

Um mínimo de sub-divisões da placa, entretanto, deve ser feita, para evitar o empobrecimento dos resultados (fig.6.6), como se viu no cap. 5.

Para o caso de lajes cogumelo, contudo, deve-se dar especial atenção para o cálculo das componentes da matriz $\underset{\sim}{\mathbf{C}}$, como se viu no cap. 4. A vinculação de nós de domínio fica, portanto, como alternativa neste caso.

\subsubsection{4 - NÓS DA BARRA LIGADOS A NÓS INTERNOS DA PLACA}

Como já mencionado na seção anterior, esta é uma opção muito utilizada por diversos pesquisadores para se fazer a análise de pavimentos. Consiste na consideração da vinculação com barras no domínio como linhas de carga (barras no plano das placas) ou como apoios discretos ou distribuídos em pequenas áreas, locados no interior das placas (barras transversais ao plano da placa). 
No cap. 2, apresentou-se a forma como se pode calcular estas regiões de carga. Menciona-se, também, a forma como se deve proceder para que pontos destas regiões sejam considerados apoios discretos ou contínuos. O desconhecimento dos valores nodais nesta região, porém, causa o aumento do número de incógnitas, conforme já discutido acima. O problema é resolvido escrevendo-se tantas equações integrais de deslocamentos e/ou rotações para os nós em questão, quantos forem necessários e de acordo com cada caso.

Como se disse, para que a vinculação destes nós influa sobre o comportamento dos outros nós da placa, as suas reações são consideradas como carregamentos discretos ou distribuídos em linha agindo sobre o domínio da placa. São, portanto, considerados na equação integral para o cálculo dos deslocamentos de qualquer nó da placa, inclusive os internos, vinculados ou não. A singularidade surge quando os deslocamentos são escritos para os nós vinculados, pois os nós campo e fonte passam a coincidir. SILVA (1996) apresenta, detalhadamente, a dedução destas integrais singulares para diversos tipos de vinculação de nós internos para as placas de Reissner.

Neste caso, então a consideração dessas novas cargas sobre a placa, transforma a eq.2.62 em:

$$
\underset{\sim}{\mathbf{H}_{\mathrm{B}}} \underset{\sim}{\mathbf{U}_{\mathrm{B}}}={\underset{\sim}{\mathbf{G}_{\mathrm{B}}} \mathbf{P}_{\mathrm{B}}}^{\mathbf{S}_{\mathrm{Bi}}}{\underset{\sim}{\mathbf{P}_{\mathrm{i}}}}^{\mathbf{B}_{\mathrm{B}}}
$$

onde o sub-índice $\underline{\mathbf{i}}$ refere-se aos nós internos vinculados e $\underline{\mathbf{B}}$ aos nós do contorno da placa. Sendo, pois, os deslocamentos nos nós internos vinculados $\left(\mathbf{U}_{\mathbf{i}}\right)$ conhecidos, as incógnitas conseqüentes deste fato, as forças $\mathbf{P}_{\mathbf{i}}$, podem ser determinadas com o auxílio das equações abaixo, conforme visto:

$$
\underset{\sim}{\mathbf{H}_{\mathrm{iB}}} \underset{\sim}{\mathbf{U}_{\mathrm{B}}}+\underset{\mathbf{I}_{\mathrm{ii}}}{\mathbf{U}_{\mathrm{i}}}={\underset{\sim}{\mathrm{iB}}}_{\sim}^{\mathbf{P}_{\mathrm{B}}}+\underset{\sim \mathrm{ii}}{\mathbf{P}_{\mathrm{i}}}+\mathbf{B}_{\mathrm{iB}}
$$


escritas para estes nós (i). As matrizes $\underset{\sim}{S_{\mathbf{B i}}}$ e $\boldsymbol{S}_{\sim}$, que consideram a influência das barras sobre o comportamento estrutural da placa, foram aqui escritas para pontos fonte no contorno e interno, respectivamente. O cálculo das suas componentes pode ser deduzido a partir do cap. 2.

Pode-se considerar os apoios em pilares locados no interior de lajes cogumelo como apoios em pontos discretos, que impedem apenas os deslocamentos verticais e desprezam a rigidez à flexão dos pilares. Porém, para qualquer tipo de estrutura de barras, basicamente, pode-se transferir a sua rigidez para a equação da placa considerando-se que:

$$
\underset{\sim}{K_{i}} \underset{\sim}{U_{i}}=\underset{\sim}{\mathbf{C}_{i}} \underset{\sim}{\mathbf{P}_{i}}+\underset{\sim}{\mathbf{F}_{\mathrm{ei}}}
$$

A partir de eq. 6.17 escrita a barra ou estrutura de barras vinculadas, obtém $\underset{\sim}{\mathbf{P}_{\mathbf{i}}}$ para substituí-lo em eqs. 6.15 e 6.16 . 


\section{6 - EXEMPLOS}

EXEMPLO I: O exemplo a seguir, foi analisado por SILVA (1996) e TANAKA \& BERCIN (1997). Ambos consideraram a influência do enrijecedor sobre a placa, como uma linha de carga distribuída, porém, o primeiro utilizou a teoria de Reissner para definir o comportamento da placa à flexão. Além disso, estes resultados não levam em conta a excentricidade do eixo da barra em relação à posição de ligação. Na tab. 6.1 estão comparados os resultados dos dois trabalhos e os obtidos nesta tese que trata as regiões equacionadas por métodos numéricos diferentes como subdomínios distintos.

Os resultados são bastante próximos, confirmando a eficiência da técnica aqui adotada.

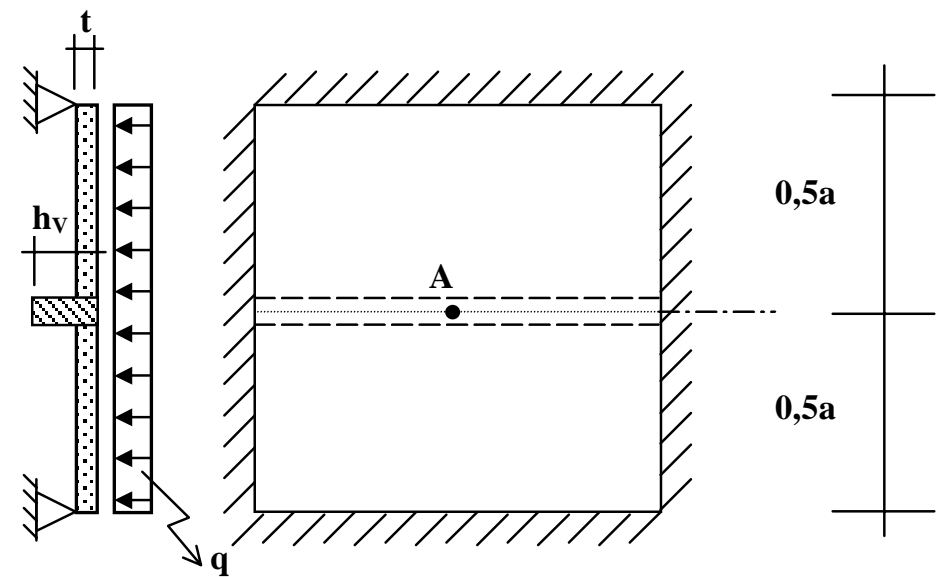

Fig. 6.7a - Exemplo I - Laje Quadrada Apoiada com enrijecedor

\begin{tabular}{|c|c|c|c|}
\hline \multicolumn{4}{|c|}{ Tab. 6.1 - Exemplo I - Resultados do exemplo-fator de deslocamento no pto. A } \\
\hline \multirow{2}{*}{ Referências } & Fator multiplicativo & \multicolumn{2}{|c|}{$\alpha_{\mathrm{A}}=\left(\mathrm{w} \cdot 10^{2} \cdot \mathrm{D}_{1}\right) /\left(\mathrm{qa}^{4}\right)$} \\
\hline & $\begin{array}{c}\text { S/ enrijecedor } \\
\alpha_{A}\end{array}$ & $\underset{\alpha_{A}}{\text { C/ enrijecedor }}\left(h_{V}=t\right)$ & C/ enrijecedor $\left(h_{V} \neq t\right)$ \\
\hline Silva (1996) & $-0,408$ & $-0,408$ & $-0,129$ \\
\hline $\begin{array}{c}\text { Tanaka \& Bercin } \\
\text { (1997) }\end{array}$ & - & - & -0.131 \\
\hline Presente trabalho & $-0,408$ & $-0,408$ & $-0,135$ \\
\hline
\end{tabular}


EXEMPLO II: Este exemplo vem ilustrar uma viga prismática, com metade do vão modelada pelo MEC e a outra metade pelo MEF (fig. 6.7b). A matriz $\underline{\mathbf{C}}$ foi adequadamente calculada para este caso de ligação, conforme comentado em 4.2.1, fig. 4.3. Esta viga foi submetida a uma carga concentrada no meio do vão e posteriormente a uma carga uniformemente distribuída no trecho $\overline{\mathbf{A C}}$.

Comparam-se os resultados de deslocamentos no ponto $\mathbf{C}$ (tab. 6.2 e 6.3) obtidos no presente trabalho, com os seus valores analíticos e com os deslocamentos calculados pela consideração da influência da rigidez da barra modelada pelo MEF na formulação do MEC para a barra $\overline{\mathbf{A C}}$. Novamente confirma-se a proximidade dos resultados.

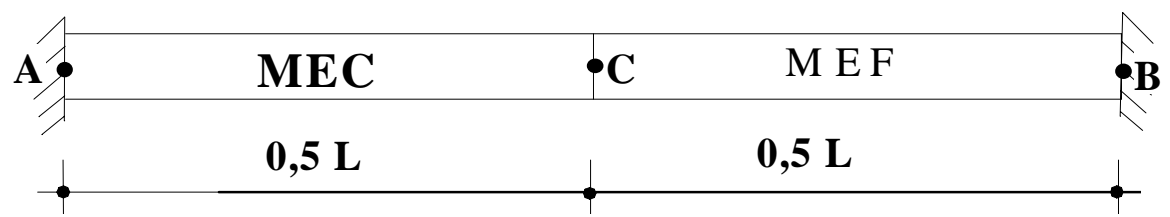

Fig. 6.7b - Viga modelada pelo MEC e MEF

Tab. 6.2 - Carga (F) Concentrada no meio do vão

\begin{tabular}{|c|c|c|c|c|}
\hline Deslocamentos & $\begin{array}{c}\text { Resultado } \\
\text { Analítico }\end{array}$ & $\begin{array}{c}\mathbf{H U}=\mathbf{G P +} \\
\mathbf{G}\left(\mathbf{C}^{-1} \mathbf{K}\right) \mathbf{U}+\mathbf{B}\end{array}$ & $\begin{array}{c}\text { Presente } \\
\text { Trabalho }\end{array}$ & $\begin{array}{c}\text { fator } \\
\text { multiplicativo }\end{array}$ \\
\hline $\mathbf{w}_{\mathbf{C}}$ & $\mathbf{1 , 0}$ & $\mathbf{1 , 0 1 7 6}$ & $\mathbf{0 , 9 9 9}$ & $\mathbf{F}(\mathbf{a L})^{3} / 24 \mathrm{EI}$ \\
\hline
\end{tabular}

Tab. 6.3 - Carga (q) Uniformemente Distribuída no Trecho $\overline{\mathrm{AC}}$

\begin{tabular}{|c|c|c|c|c|}
\hline Deslocamentos & $\begin{array}{c}\text { Resultado } \\
\text { Analítico }\end{array}$ & $\begin{array}{c}\mathbf{H U = G P +} \\
\mathbf{G}\left(\mathbf{C}^{-1} \mathbf{K}\right) \mathbf{U}+\mathbf{B}\end{array}$ & $\begin{array}{c}\text { Presente } \\
\text { Trabalho }\end{array}$ & $\begin{array}{c}\text { fator } \\
\text { multiplicativo }\end{array}$ \\
\hline $\mathbf{w}_{\mathbf{C}}$ & $\mathbf{1 , 0}$ & $\mathbf{1 , 0 3 0 0}$ & $\mathbf{1 , 0 3 0 0}$ & $\mathbf{q}(\mathbf{a L})^{4} / \mathbf{4 8 E I}$ \\
\hline$\theta_{\mathbf{C}}$ & $\mathbf{1 , 0}$ & $\mathbf{1 , 0 9 1 0}$ & $\mathbf{1 , 0 9 1 7}$ & $\mathbf{q}(\mathbf{a L})^{3} / 96 E \mathbf{E I}$ \\
\hline
\end{tabular}




\section{7 - EFEITO DE MEMBRANA - ESTADO PLANO DE TENSÃO}

Nos capítulos anteriores, foram expostas, separadamente, as formulações para flexão de placas, o estado plano de tensões e a análise de esforços em barras. Neste capítulo, até então, foram abordados os detalhes e aproximações adotadas para a união entre os elementos de superfície e lineares, de acordo com os métodos numéricos utilizados para a análise de cada um.

Da forma como foi elaborado o acoplamento até agora, seja entre sub-regiões formuladas pelo MEC ou entre regiões do MEC e do MEF, tudo se comporta como se os planos das superfícies neutras das placas ou os seus traços sobre o plano da interface com os eixos neutros das vigas (quando existentes) coincidissem. Estariam as placas, então, trabalhando apenas a flexão, quando solicitados por carregamento vertical. Analisando-se o multi-domínio desta forma, os resultados obtidos dirão respeito a situações como as ilustradas nas figs. 6.8 a 6.10 (a partir de agora, as espessuras das placas serão representadas por $\mathbf{h}_{\mathbf{p}}$ ).

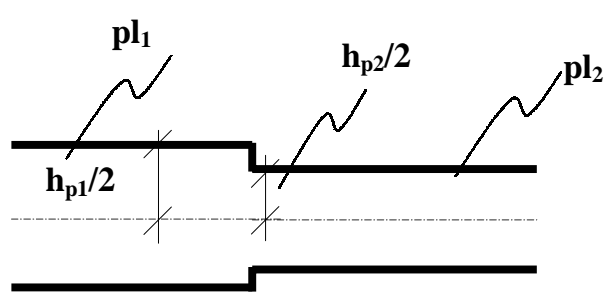

Fig. 6.8 - Sub-regiões $\left(h_{1} \neq h_{2}\right)$ com superfícies neutras no mesmo nível;apoio no nível das superfícies neutras

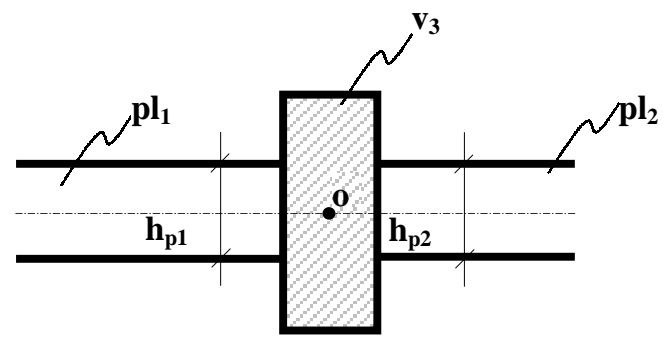

Fig. 6.9 - Sub-regiões com $h_{p 1}=h_{p 2}$, apoios no nível da superfície neutra e do eixo neutro da viga; viga apoiada ou não (quando sim, no nível do eixo neutro). 


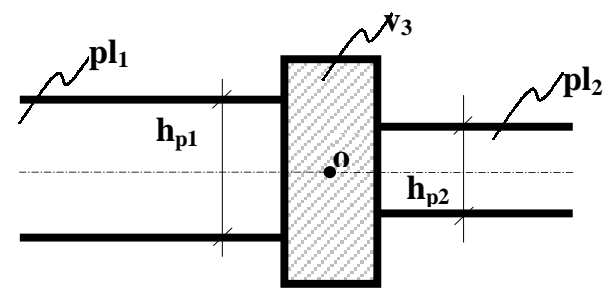

Fig. 6.10 - Idem à Fig. 6.9, $\operatorname{com} h_{p 1} \neq h_{p 2}$

Na prática, esta coincidência não ocorre com freqüência. VENTURINI(1988) alerta para o fato de que, durante a análise de pavimentos deve-se levar em conta a excentricidade entre o eixo neutro da barra e a superfície neutra da placa, ou ainda, que a excentricidade entre as superfícies neutras de placas adjacentes, seja levado em conta (fig.(6.11)). Surge, assim, o esforço normal, paralelo ao plano médio da placa que é combinado com a flexão causada pelo carregamento transversal que ela suporta. Considera-se que os apoios das placas e barras estão ao nível da placa de menor altura, chamada de placa referência.
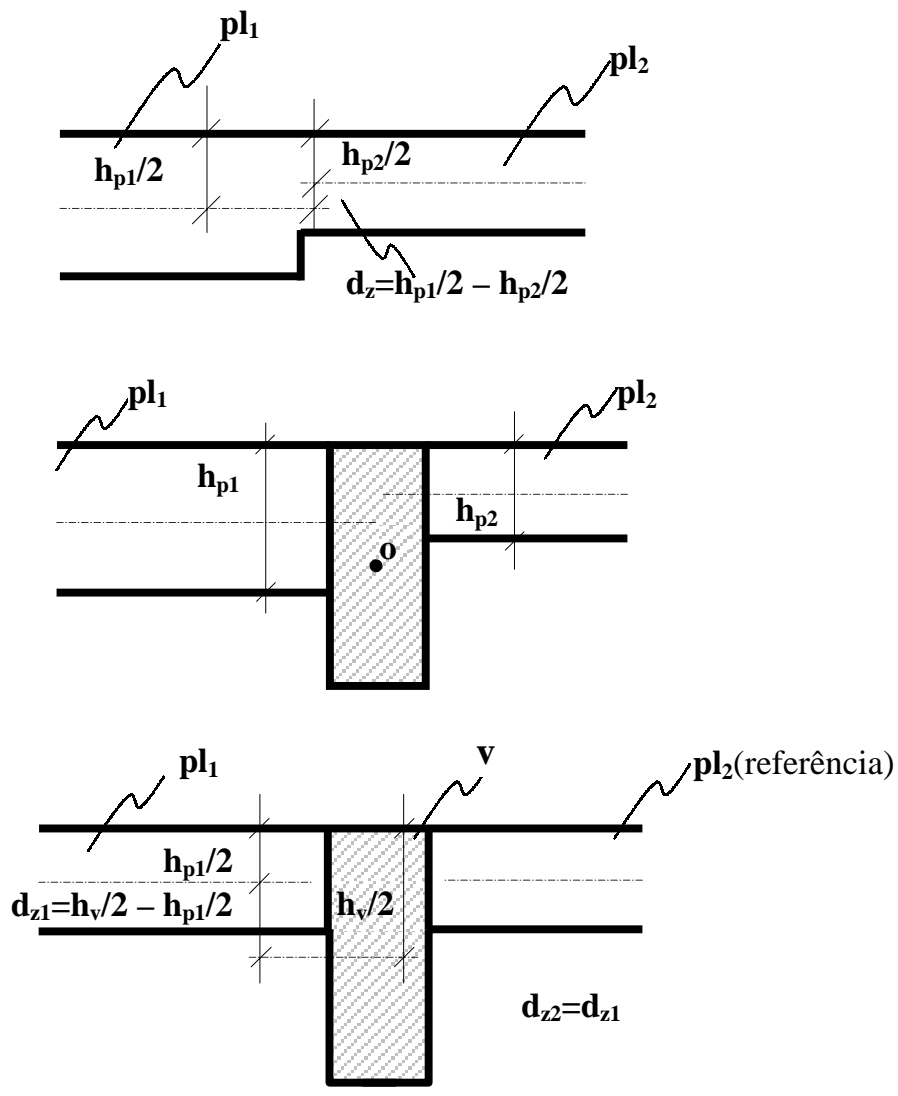

Fig. 6.11 - Apoios ao nível da placa referência $\left(\mathbf{p l}_{2}\right)$ 
No presente estudo, a exemplo do método usado por PALERMO JR.(1989), estes efeitos são considerados individualmente para cada elemento estrutural. Se o carregamento sobre a estrutura for apenas vertical, a combinação dos dois fenômenos surgirá apenas se houver excentricidade entre as superfícies e eixos neutros.

\subsection{1 - COMBINAÇÃO DOS ELEMENTOS PLACA E CHAPA}

É oportuno relembrar que, para a maneira com que foram concebidas as formulações que descrevem o comportamento dos elementos estruturais de superfície até então, tanto para a flexão de placas, quanto no estado plano de tensão, não há interdependência entre variáveis envolvidas pelos dois fenômenos. Deve-se prever, porém, a possibilidade do surgimento de uma excentricidade entre elementos estruturais que introduza a combinação entre os dois fenômenos (fig. 6.12).
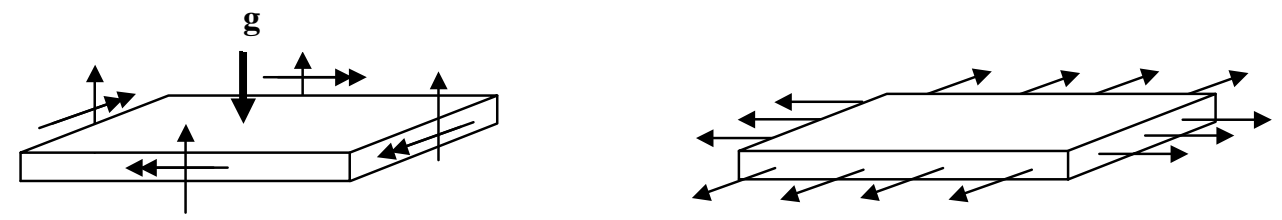

Fig. 6.12 - Ações e efeitos sobre elementos placa e chapa

Aplicando-se as equações integrais para o cálculo das variáveis para os estados de flexão de placas (eq. 2.43 ) e para o EPT (eq. 3. 18) sobre os mesmos $\mathbf{N}_{\text {n }}$ pontos de contorno das sub-regiões de elementos de superfícies, obtém-se um total de equações, portanto, de duas vezes o seu número $\left(2 \mathbf{N}_{\mathrm{n}}\right.$, relativos à placa) mais novamente $2 \mathbf{N}_{\mathrm{n}}$ (relativos à chapa) quando se pretende ligar duas regiões de placas. Quando a ligação é entre regiões de placas e barras o total de equações passa a ser $3 \mathbf{N}_{\mathrm{n}}$ (relativos à placa) mais $2 \mathbf{N}_{\mathrm{n}}$ (relativos à chapa). $\mathrm{O}$ sistema de equações resultante terá a forma da eq. 2.62, sendo que, agora, as componentes dos vetores de 
deslocamentos e esforços, para o caso de um nó i do contorno das sub-regiões de superfície são:

$$
\begin{aligned}
& \underset{\sim_{i}}{\mathbf{U}^{T}}=\left\{\begin{array}{llll}
u_{n} & u_{s} & w & \theta_{n}
\end{array}\right\}_{i} \\
& \underset{\sim_{i}}{\mathbf{P}_{i}^{T}}=\left\{\begin{array}{lllll}
N & S & V_{n} & m_{n}
\end{array}\right\}_{i}
\end{aligned}
$$

se a ligação será entre placas, $\mathbf{N}_{\mathbf{i}}$ e $\mathbf{S}_{\mathbf{i}}$ sendo as componentes nas direções normal e tangente ao contorno em $\mathbf{i}$, calculados a partir de $\mathbf{p}_{\mathbf{1}}$ e $\mathbf{p}_{\mathbf{2}}$. $\mathbf{u}_{\mathbf{n i}}$ e $\mathbf{u}_{\mathbf{s i}}$ são os deslocamentos correspondentes a $\mathbf{N}_{\mathbf{i}}$ e $\mathbf{S}_{\mathbf{i}}$, respectivamente. Ainda, conforme visto em 6.2 e 6.3 :

$$
\begin{aligned}
& \underset{\sim}{\mathbf{U}_{i}^{T}}=\left\{\begin{array}{lllll}
u_{1} & \mathbf{u}_{2} & \mathbf{w}_{3} & \theta_{4} & \theta_{5}
\end{array}\right\}_{i} \\
& \underset{\sim}{\mathbf{P}_{i}^{T}}=\left\{\begin{array}{llllll}
p_{1} & \mathbf{p}_{2} & \mathbf{Q}_{3} & \mathbf{m}_{4} & \mathbf{m}_{5}
\end{array}\right\}_{i}
\end{aligned}
$$

para ligação a ser feita entre placas e barras. Observe-se que se abordam aqui apenas os vetores e matrizes gerados para os elementos de superfície. Já os gerados para as regiões de barras, como se viu em 6.4, não sofrem modificações neste sentido.

As matrizes $\underset{\sim}{\mathbf{H}}$ e $\underset{\sim}{\mathbf{G}}$, cujos componentes são calculados pela integração das citadas equações(eqs. 2.43 e 3.18), apresentam o seguinte aspecto para um determinado nó i para ligações entre placas (MEC/MEC):

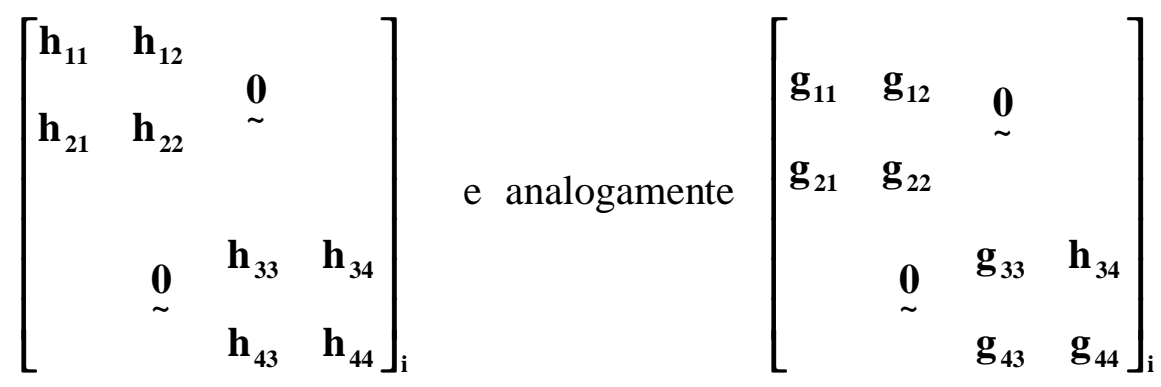


ou, para ligações entre placas e barras (MEC/MEF)

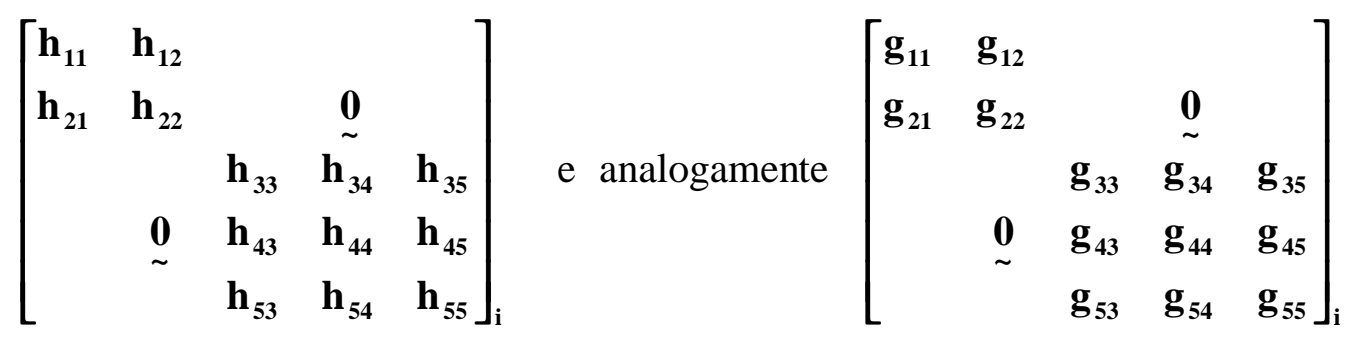

O vetores $\underset{\sim}{\mathbf{U}}$ e $\underset{\sim}{\mathbf{P}}$ contém as variáveis de deslocamentos e de forças de superfície, respectivamente que serão determinadas com o auxílio das condições de contorno, no caso de um domínio isolado em equilíbrio. Para o caso de sub-regiões acopladas, onde há interfaces, para cada nó i sobre estas linhas, surgem novas equações que permitem resolver o problema. Supondo inicialmente o exemplo de apenas duas subregiões acopladas e sem influências externas, sabe-se que estas equações surgem da verificação da compatibilidade de deslocamentos e do estabelecimento do equilíbrio nos nós de interface. Às equações já vistas no cap. 5 (que estabelecem a compatibilidade entre os deslocamentos verticais e as rotações dos nós de interface das sub-regiões, bem como o equilíbrio entre os esforços cortantes e momentos distribuídos), juntam-se as equações abaixo, envolvendo as variáveis relativas ao EPT. Para ligações entre sub-regiões do tipo MEC/MEC, elas são:

$$
\begin{aligned}
& \mathbf{u}_{\mathrm{ni}}^{\mathrm{I}}=-\mathbf{u}_{\mathrm{ni}}^{\mathrm{II}} \\
& \mathbf{u}_{\mathrm{si}}^{\mathrm{I}}=-\mathbf{u}_{\mathrm{si}}^{\mathrm{II}} \\
& \mathbf{N}_{\mathrm{i}}^{\mathrm{I}}=\mathbf{N}_{\mathrm{i}}^{\mathrm{II}} \\
& \mathbf{S}_{\mathbf{i}}^{\mathrm{I}}=\mathbf{S}_{\mathbf{i}}^{\mathrm{II}}
\end{aligned}
$$

e para ligações do tipo MEC/MEF: 


$$
\begin{aligned}
& \mathbf{u}_{1 \mathbf{i}}^{\mathrm{I}}=\mathbf{u}_{1_{\mathrm{i}}}^{\mathrm{II}} \\
& \mathbf{u}_{2 \mathrm{i}}^{\mathrm{I}}=\mathbf{u}_{2 \mathrm{i}}^{\mathrm{II}} \\
& \mathbf{p}_{1_{\mathrm{i}}}^{\mathrm{I}}=-\mathbf{p}_{1_{\mathrm{i}}}^{\mathrm{II}} \\
& \mathbf{p}_{2 \mathrm{i}}^{\mathrm{I}}=-\mathbf{p}_{2_{\mathrm{i}}}^{\mathrm{II}}
\end{aligned}
$$

Para estender-se ao caso de várias sub-regiões unidas numa mesma interface e/ou no caso de interface com condições de contorno conhecidas, deve-se seguir as deduções feitas no cap. 5 para estes casos.

\subsection{2 - IDEALIZAÇÃO DO MODELO A PARTIR DO PTV}

CORRÊA(1991) expôs passo a passo a técnica de translação de coordenadas para efeito da elaboração da consideração de trechos rígidos na estrutura. Usando a mesma técnica baseada no princípio dos trabalhos virtuais (PTV), será aqui elaborado o efeito de membrana que surge em presença de excentricidade $\left(\mathbf{d}_{\mathbf{z}}\right)$ entre as superfícies neutras das placas e barras (fig.(6.13)).
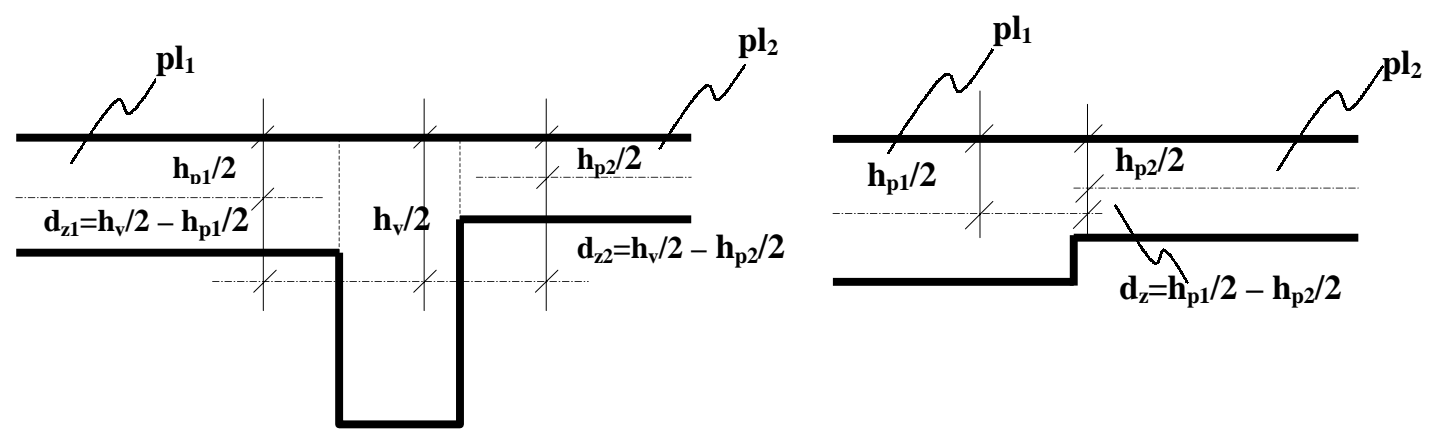

Fig. 6.13 - Excentricidades entre os planos e eixos neutros

Conforme o PTV, os trabalhos realizados por dois sistemas de forças estaticamente equivalentes, aos quais correspondem sistemas de deslocamento de pontos do corpo, são iguais quando é dado um deslocamento virtual de corpo rígido, a saber (fig.(6.14)): 


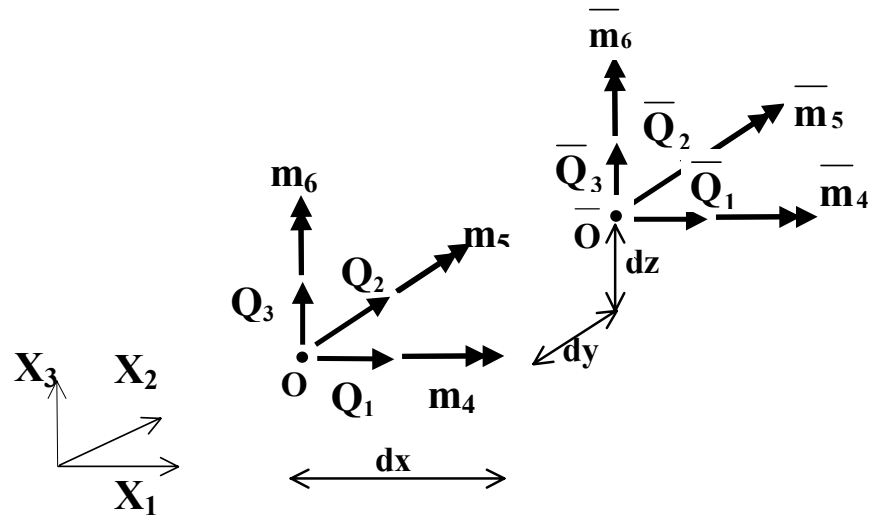

Fig. 6.14 - Sistemas de forças equivalentes

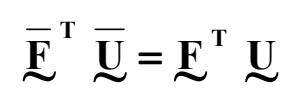

sendo, para o problema 3-D, num determinado ponto i:

$$
\begin{aligned}
& \stackrel{\mathbb{L}}{\mathrm{T}}^{\mathrm{T}}=\left\{\mathbf{V}_{\mathrm{x}} \mathbf{V}_{\mathrm{y}} \mathbf{V}_{\mathrm{z}} \mathbf{M}_{\mathrm{x}} \mathbf{M}_{\mathrm{y}} \mathbf{M}_{\mathrm{z}}\right\} \\
& \underline{\sim}^{\mathrm{T}}=\left\{\begin{array}{llll}
\boldsymbol{u} \mathbf{v} \mathbf{w} & \boldsymbol{\theta}_{\mathrm{x}} & \boldsymbol{\theta}_{\mathrm{y}} & \boldsymbol{\theta}_{\mathrm{z}}
\end{array}\right\}
\end{aligned}
$$

que representam os sistemas de força e deslocamentos, respectivamente. Como $\underset{\mathbf{E}}{\mathbf{L}} \underset{\mathbf{\mathbf { E }}}{\sim}$ são equivalentes,

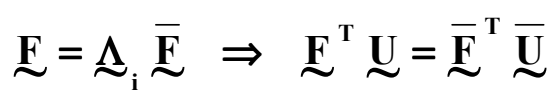

donde se conclui que

$$
\overline{\mathbf{U}}=\underline{\sim}_{\mathbf{i}}^{\mathrm{T}} \mathbf{U}
$$


que são as correlações entre variáveis estáticas (eq. 6.26) e cinemáticas (eq. 6.27), respectivamente. Conforme se pode observar em CORRÊA(1991), a matriz de transformação ${\underset{\sim}{i}}_{i}$ das componentes de forças e deslocamentos entre sistemas de eixos coordenados paralelos entre si, para o problema 3-D contém os valores

$$
\underline{\sim}=\left[\begin{array}{cc}
\mathbf{I}_{3} & \mathbf{0}_{3} \\
\boldsymbol{\Omega}_{\mathrm{i}_{3}} & \underline{\mathbf{I}}_{3}
\end{array}\right]
$$

onde $\underset{\mathbf{I}_{3}}{\sim}, \mathbf{0}_{3}$ e $\Omega_{\mathrm{i} 3}$ são, respectivamente, as matrizes de ordem três identidade, nula e:

$$
\stackrel{\Omega}{i 3}_{\sim}=\left[\begin{array}{ccc}
0 & -d_{z} & d_{y} \\
d_{z} & 0 & -d_{x} \\
-d_{y} & d_{x} & 0
\end{array}\right]
$$

sendo $\boldsymbol{\Omega}_{\mathrm{i} 3}^{\mathrm{T}}=-\boldsymbol{\Omega}_{\mathrm{i} 3}$ e $\mathbf{d}_{\mathbf{x}}, \mathbf{d}_{\mathbf{y}}$ e $\mathbf{d}_{\mathbf{z}}$ as componentes da distância entre os dois sistemas (vide fig. 6.14).

As influências da translação das coordenadas dos pontos dos elementos das barras e/ou das placas sobre as equações de equilíbrio que descrevem seu comportamento, serão avaliados nas seções que seguem. Serão ser analisados tanto o caso de placas acopladas com excentricidade entre os planos das suas superfícies neutras, como a união de placas e barras de superfícies e eixos neutros não coplanares paralelos.

\subsubsection{1-LIGAÇÃO ENTRE REGIÕES DE PLACAS (MEC/MEC)}

Originalmente, conforme visto no último item, os efeitos da flexão e do EPT sobre os elementos estruturais de superfície ocorrem individualmente, de acordo com 
a natureza da solicitação a eles imposta. Em 6.7, comentou-se que a existência da excentricidade entre os planos neutros das placas acopladas provoca a combinação dos dois fenômenos. Será agora, portanto, orientado o desenvolvimento da formulação para atender a este caso.

O sistema de equações que permite descrever o comportamento à flexão e ao EPT de placas se refere originalmente à superfície neutra de cada uma delas (superfície esta que passa pelo ponto $\mathbf{O}$ - fig. 6.16):

$$
\underset{\sim}{\mathbf{H}_{\mathbf{o}} \mathbf{U}_{\mathbf{0}}}=\underset{\sim}{\mathbf{G}_{\mathbf{0}} \mathbf{P}_{\mathbf{O}}}
$$

escrita para cada placa.

Neste caso, como se sabe, os esforços $\underset{\sim}{\mathbf{P}_{\mathbf{O}}}$ e deslocamentos $\underset{\sim}{\mathbf{U}_{\mathbf{O}}}$, relativos ao EPT, se referem ao sistema de coordenadas paralelo a $\mathbf{x}_{1}$ e $\mathbf{x}_{2}$, conforme elaborado no cap. 3. Eles podem ser expressos em relação a coordenadas locais, isto é, em relação aos versores normal $\overrightarrow{\mathbf{n}}$ e tangente $\overrightarrow{\mathbf{s}}$, que para um determinado ponto do contorno a transformação é feita através das relação (fig. 6.15):

$$
\left\{\begin{array}{l}
\mathbf{p}_{1} \\
\mathbf{p}_{2}
\end{array}\right\}=\left[\begin{array}{ll}
n_{1} & \mathbf{s}_{1} \\
n_{2} & \mathbf{s}_{2}
\end{array}\right]\left\{\begin{array}{l}
\mathbf{N} \\
\mathbf{S}
\end{array}\right\}
$$

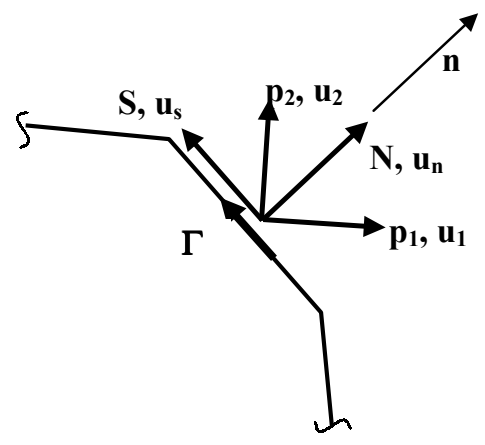

FIG. 6.15 - Componentes normal e tangente de esforços, gerados pelo EPT 
sendo $\mathbf{n}_{\mathbf{i}}$ e $\mathbf{s}_{\mathbf{i}}$ os cossenos diretores de $\overrightarrow{\mathbf{n}}$. Analogamente, determinam-se os deslocamentos pela relação:

$$
\left\{\begin{array}{l}
u_{1} \\
u_{2}
\end{array}\right\}=\left[\begin{array}{ll}
n_{1} & s_{1} \\
n_{2} & s_{2}
\end{array}\right]\left\{\begin{array}{l}
u_{n} \\
u_{s}
\end{array}\right\}
$$

Para todos os $\mathbf{N}_{\mathbf{n}}$ pontos do contorno da placa, a transformação para os esforços fica:

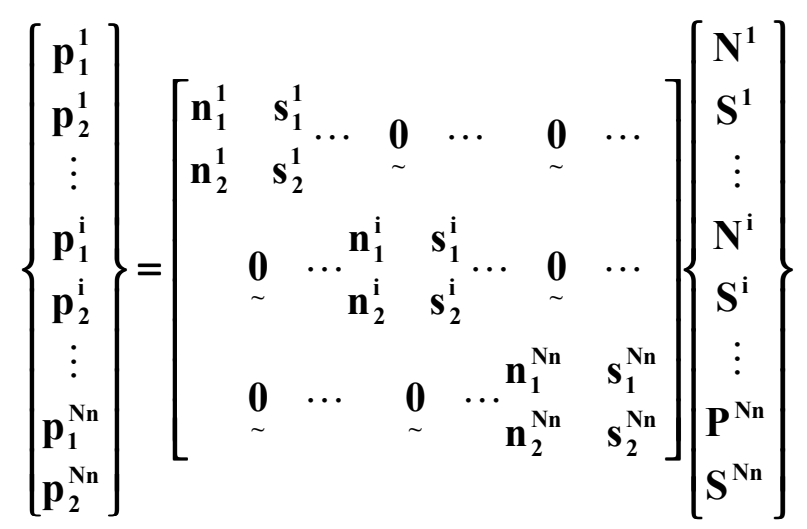

sendo $\mathbf{n}_{\mathbf{m}}^{\mathbf{i}}$ referente à direção $\underline{\mathbf{m}}$, igual a 1 ou $\mathbf{2}$, no nó $\underline{\mathbf{i}}$. Deve-se agir da mesma forma para os deslocamentos.

Esta transformação é feita pelo menos para todas os nós de interface entre subregiões. Para os outros nós de contorno, será feita apenas de houver interesse em conhecer os esforços e deslocamentos do EPT na direção normal e tangente ao contorno nestes pontos.

Feito isto, chame-se de $\mathbf{A}$ o nó de contorno da placa $\mathbf{p l}_{\mathbf{2}}$ ao nível da superfície neutra da placa referencial $\left(\mathbf{p l}_{\mathbf{1}}\right)$. Este nó está relacionado ao nó $\mathbf{O}$ que se situa ao nível da superfície neutra de $\mathbf{p l}_{2}$. A distância (ou excentricidade) entre $\mathbf{A}$ e $\mathbf{O}$ vale $\underline{\mathbf{e}}$ (fig. 6.16), suposta constante ao longo da linha da interface.

Analisem-se, inicialmente, os esforços na interface, no contorno da placa $\mathbf{p l}_{\mathbf{2}}$. Nos pontos $\mathbf{A}$ e $\mathbf{O}$, estes esforços relativos à normal ao contorno nestes pontos, estão 
representados na fig. 6.16. Para se escrever, a equação de equilíbrio do sistema de forças de origem em A e que é equivalente ao de origem em $\mathbf{O}$, utiliza-se o PTV (6.7.2) ou recorre-se diretamente às relações expostas naquela figura:
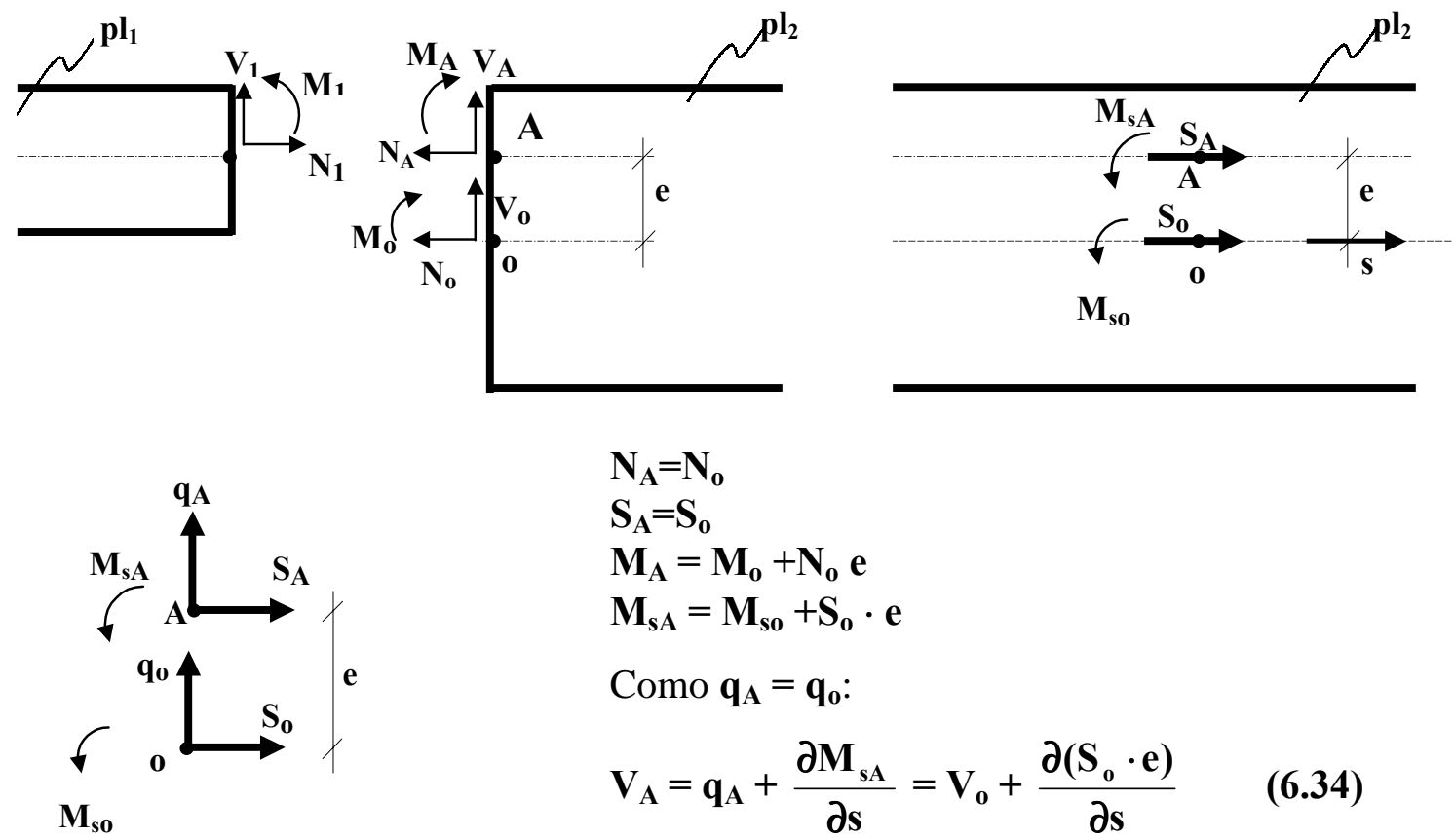

$$
\begin{aligned}
& \mathbf{N}_{\mathrm{A}}=\mathbf{N}_{\mathbf{o}} \\
& \mathbf{S}_{\mathrm{A}}=\mathbf{S}_{\mathbf{o}} \\
& \mathbf{M}_{\mathrm{A}}=\mathbf{M}_{\mathbf{0}}+\mathbf{N}_{\mathbf{o}} \mathbf{e} \\
& \mathbf{M}_{\mathbf{s A}}=\mathbf{M}_{\mathbf{s o}}+\mathbf{S}_{\mathbf{o}} \cdot \mathbf{e} \\
& \text { Como } \mathbf{q}_{\mathrm{A}}=\mathbf{q}_{\mathbf{o}}: \\
& \mathbf{V}_{\mathrm{A}}=\mathbf{q}_{\mathrm{A}}+\frac{\partial \mathbf{M}_{\mathrm{sA}}}{\partial \mathbf{s}}=\mathbf{V}_{\mathbf{o}}+\frac{\partial\left(\mathbf{S}_{\mathbf{o}} \cdot \mathbf{e}\right)}{\partial \mathbf{s}}
\end{aligned}
$$

Fig. 6.16 - Esforços em um ponto da região da interface de ligação entre duas placas

de onde passa-se a chamar $\Delta \mathbf{V}_{\mathbf{o}}$ o acréscimo ao esforço $\mathbf{V}_{\mathbf{0}}$, que vale:

$$
\Delta \mathbf{V}_{\mathrm{o}}=\frac{\partial\left(\mathrm{S}_{\mathrm{o}} \cdot \mathrm{e}\right)}{\partial \mathrm{s}}
$$

Para calcular $\Delta \mathbf{V}_{\mathbf{0}}$, pode-se escrever a componente $\mathbf{S}_{\mathbf{0}}$ como uma aproximação dos valores nodais $\mathbf{S}_{\mathbf{i}}$ deste elemento, sendo $\underline{\mathbf{O}}$ um nó sobre um elemento $\boldsymbol{\Gamma}_{\mathbf{j}}$, de comprimento $\ell$ e $\mathbf{i}=\mathbf{1}, \mathbf{2}$ ou $\mathbf{3}$, que são os nós inicial, do meio e final do elemento:

$$
\mathbf{S}_{0}=\phi_{1} \mathbf{S}_{1}+\phi_{2} \mathbf{S}_{2}+\phi_{3} \mathbf{S}_{3}
$$




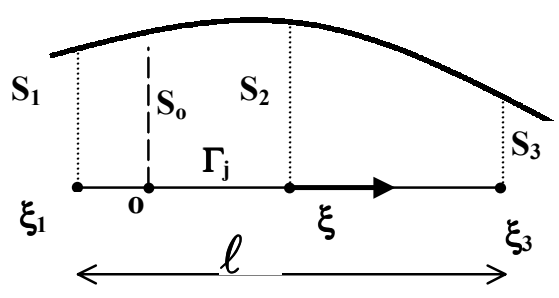

Fig. 6.17 - Aproximação da componente tangencial $S_{0}$

Sendo $\phi_{\mathrm{i}}$ as funções aproximadoras já apresentadas no cap. 2 (eq. 2.68).

Daí, pode-se desenvolver a eq.6.35, transformando-a em:

$$
\Delta \mathbf{V}_{\mathrm{o}}=\mathbf{e} \cdot \sum_{\mathrm{i}=1}^{3} \frac{\partial \phi_{\mathrm{i}}}{\partial \mathrm{s}} \mathbf{S}_{\mathrm{i}}
$$

para aproximação quadrática das variáveis de contorno. Ainda, sabe-se também que:

$$
\frac{\partial \phi_{i}}{\partial s}=\frac{\partial \phi_{i}}{\partial \xi} \frac{\partial \xi}{\partial s}=\frac{2}{\ell} \frac{\partial \phi_{i}}{\partial \xi} \quad i=1,3
$$

Para o cálculo de $\Delta \mathbf{V}_{\mathbf{0}}$, quando o nó $\underline{\mathbf{O}}$ é um nó interno do elemento, a transformação acima é suficiente. Para os nós de extremidade de um elemento, existem duas outras condições a serem consideradas. A primeira é quando o nó $\underline{\mathbf{0}}$ de extremidade pertence a um elemento com descontinuidade neste nó (fig. 6.18). Daí,:

$$
\Delta \mathbf{V}_{\mathbf{o}}=\Delta \mathbf{V}_{\mathbf{o}(\text { ant })}
$$

se o nó é do extremo final do elemento descontínuo, sendo $\Delta \mathbf{V}_{\mathbf{o}(\mathbf{a n t})}$ relativo ao elemento anterior a este nó. Se o nó é do extremo inicial deste tipo de elemento,

$$
\Delta \mathbf{V}_{\mathbf{o}}=\Delta \mathbf{V}_{\mathbf{o}(\text { post })}
$$


sendo $\Delta \mathbf{V}_{\mathbf{o}(\text { post) }}$ relativo ao elemento posterior ao nó.

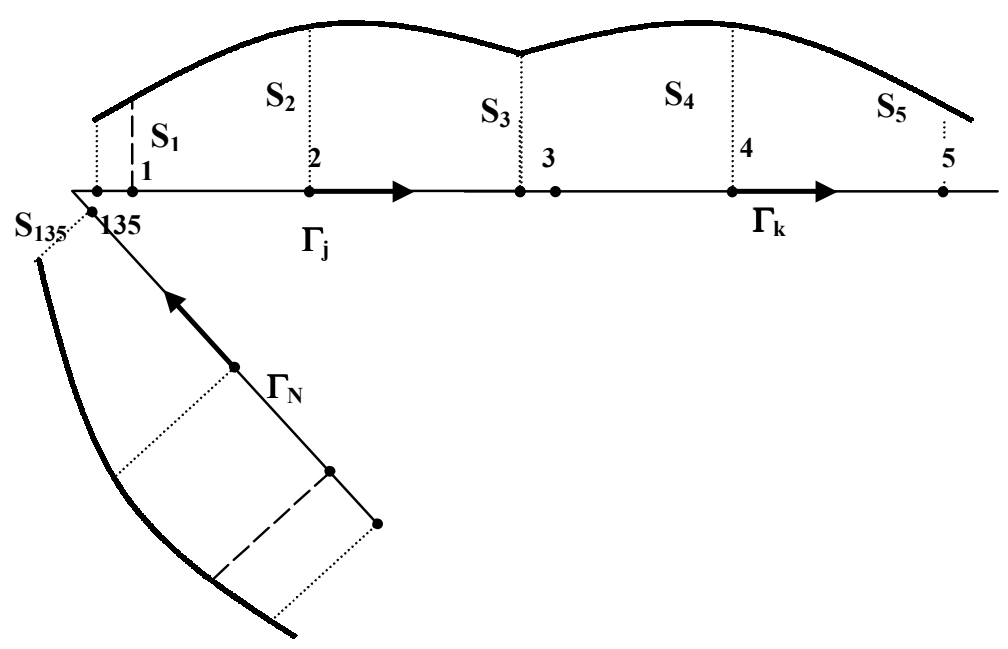

Fig. 6.18 - Elementos contínuos e descontínuos.

A segunda possibilidade é o nó $\underline{\mathbf{O}}$ coincidente com a extremidade de elemento contínuo (fig. 6.18). Neste caso, deve-se considerar a contribuição dos resultados calculados para os dois elementos aos quais pertence da forma:

$$
\Delta \mathrm{V}_{\mathrm{o}}=\frac{1}{2}\left(\Delta \mathrm{V}_{\mathrm{o}(\mathrm{ant})}+\Delta \mathrm{V}_{\mathrm{o}(\mathrm{post})}\right)
$$

Com o auxílio das eqs. $\mathbf{2 . 6 8}$ e 6.38, pode-se calcular as derivadas da eq. 6.37, obtendo:

$$
\begin{aligned}
& \frac{\partial \phi_{1}(\mathbf{O})}{\partial \xi}=\frac{\left(\xi_{3}-2 \xi\right)}{\xi_{1}\left(\xi_{3}-\xi_{1}\right)} \\
& \frac{\partial \phi_{2}(\mathbf{O})}{\partial \xi}=-\frac{\left(\xi_{3}+\xi_{1}\right)}{\xi_{1} \xi_{3}}+\frac{2 \xi}{\xi_{1} \xi_{3}} \\
& \frac{\partial \phi_{3}(\mathbf{O})}{\partial \xi}=\frac{\left(\xi_{1}-2 \xi_{)}\right)}{\xi_{3}\left(\xi_{1}-\xi_{3}\right)}
\end{aligned}
$$

para elemento descontínuo, onde $\xi$ é a coordenada genérica do nó $\mathbf{O}$ e: 


$$
\begin{aligned}
& \frac{\partial \phi_{1}(O)}{\partial \xi}=-\frac{1}{2}+\xi \\
& \frac{\partial \phi_{2}(O)}{\partial \xi}=-2 \xi \\
& \frac{\partial \phi_{3}(O)}{\partial \xi}=\frac{1}{2}+\xi
\end{aligned}
$$

para o nós de elementos contínuos, o que se obtém da eq. $\mathbf{6 . 4 2}$ fazendo $\boldsymbol{\xi}_{1}=-\mathbf{1}, \boldsymbol{\xi}_{2}=\mathbf{0}$ e $\boldsymbol{\xi}_{3}=\mathbf{1}$. Deve-se sempre lembrar de dividir os valores de $\partial \phi_{1}(\mathbf{O}) / \partial \xi$ e/ou $\partial \phi_{3}(\mathbf{O}) / \partial \xi$ por dois, conforme eq. $\mathbf{6 . 4 1}$.

Analisem-se, agora os deslocamentos relacionados àqueles esforços da interface. Da mesma maneira, pode-se escrever a relação entre os componentes de deslocamento no sistema de origem em $\mathbf{A}$ e o de origem em $\mathbf{O}$ conforme a fig. 6.19:
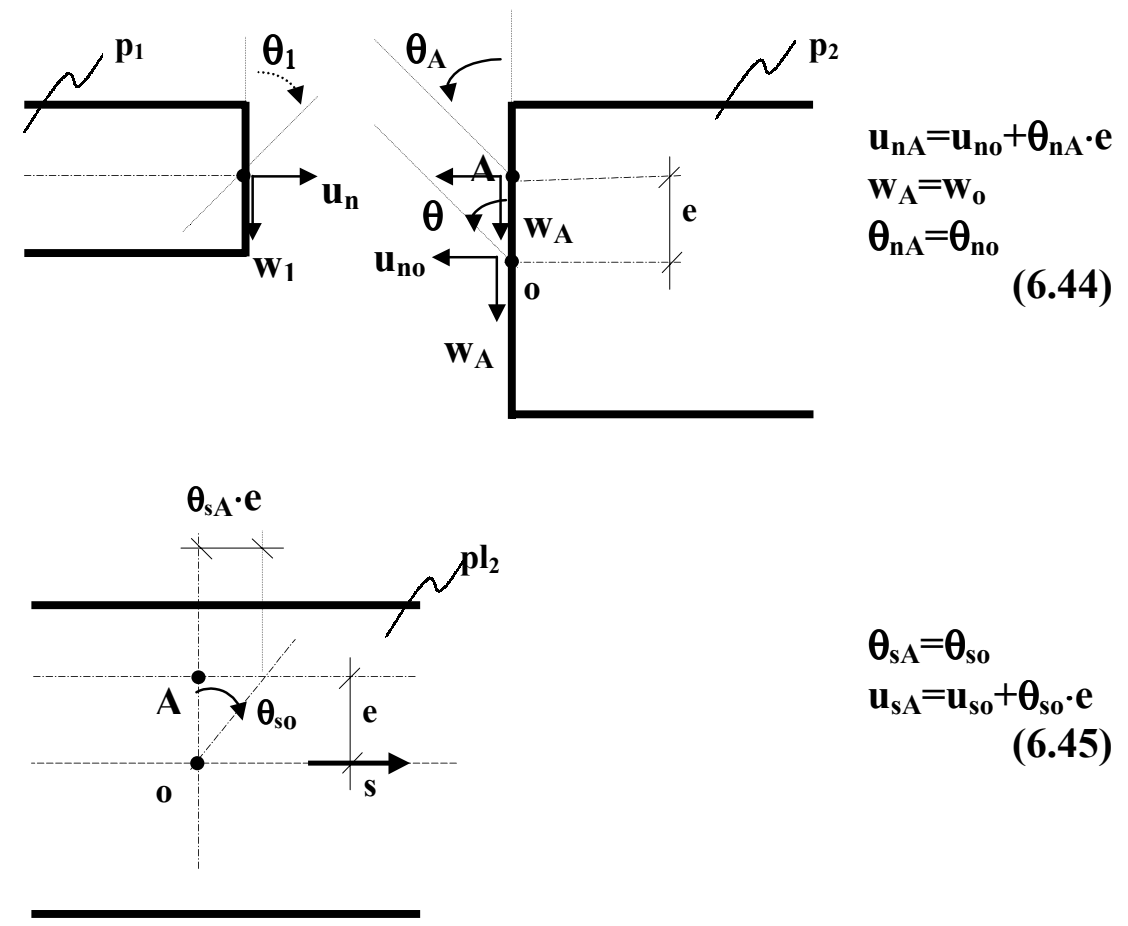

$$
\begin{aligned}
& \theta_{\mathrm{sA}}=\theta_{\text {so }} \\
& \mathbf{u}_{\mathrm{sA}}=\mathbf{u}_{\mathrm{so}}+\theta_{\mathrm{so}} \cdot \mathrm{e}
\end{aligned}
$$

Fig. 6.19 - Deslocamentos em um ponto da região da interface de ligação entre duas placas

A variação no deslocamento na direção tangente $\left(\Delta \mathbf{u}_{\mathbf{s o}}=\boldsymbol{\theta}_{\mathbf{s o}} \cdot \mathbf{e}\right)$, a exemplo do que se deduziu para a componente tangente $\mathbf{S}_{\mathbf{0}}$, pode ser reescrita como: 


$$
\Delta \mathbf{u}_{\mathrm{s} 0}=\mathrm{e} \cdot \frac{\partial \mathrm{w}}{\partial \mathrm{s}}=\mathrm{e} \sum_{\mathrm{i}=1}^{3} \frac{\partial \phi_{\mathrm{i}}}{\partial \mathrm{s}} \mathbf{w}_{\mathrm{i}}
$$

para $\mathbf{w}$ escrito como uma aproximação dos valores nodais $\mathbf{w}_{\mathbf{i}}$ do elemento ao qual pertence $\underline{\underline{\mathbf{O}}}$, isto é (fig. 6.20):

$$
\mathbf{w}_{\mathbf{0}}=\phi_{1} \mathbf{w}_{1}+\phi_{2} \mathbf{w}_{2}+\phi_{3} \mathbf{w}_{3}
$$

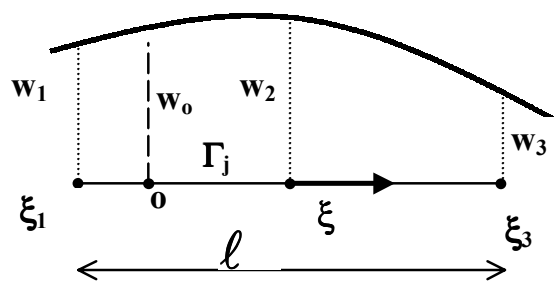

Fig. 6.20 - Aproximação da componente de deslocamento $w_{0}$

As derivadas $\frac{\partial \phi_{i}}{\partial s}$, com $\mathbf{i}=\mathbf{1 , 3}$, são calculadas através das eq. 6.38 e $\mathbf{6 . 4 2}$.

Para o cálculo de $\Delta \mathbf{u}_{\mathbf{s o}}$, valem também as condições observadas na determinação de $\Delta \mathbf{V}_{\text {so }}$ para o caso do nó $\mathbf{O}$ estar situado no interior ou nas extremidades do elemento em estudo, em presença ou não de descontinuidade.

Observe-se que todos os nós de contorno (de interface ou não) terão as variáveis escritas em relação à superfície neutra da placa referência (a placa $\mathbf{p l}_{1}$ no exemplo das figs. 6.16 e 6.19). Na região da interface, $\mathbf{N}_{\mathbf{A}}$ e $\mathbf{S}_{\mathbf{A}}\left(\right.$ ou $\mathbf{p}_{1}^{\mathrm{A}}$ e $\left.\mathbf{p}_{2}^{\mathbf{A}}\right), \mathbf{V}_{\mathrm{A}}$ e $\mathbf{M}_{\mathrm{A}}$ são desconhecidas, bem como as variáveis $\mathbf{u}_{\mathbf{n A}}$ e $\mathbf{u}_{\mathrm{sA}}, \mathbf{w}_{\mathrm{A}}$ e $\boldsymbol{\theta}_{\mathbf{n A}}$. Como se sabe, estas incógnitas são calculadas através da técnica das sub-regiões (aplicada, inclusive, no elemento de chapa, conforme visto em 6.7.1) pelo estabelecimento do equilíbrio e compatibilidade de deslocamentos nos seus pontos. 
Transfere-se, desta forma, o referencial para elaboração das equações de deslocamentos de todos os nós cada sub-região. Isto é, as variáveis dos deslocamentos dos nós ao nível de suas respectivas superfícies neutras passam a representar os deslocamentos dos nós ao nível da superfície neutra da placa referência.

São construídas, assim matrizes que promovem esta transferência, $\left[\overline{\mathbf{T}}_{\mathbf{u}}\right]$ e $\left[\overline{\mathbf{T}}_{\mathbf{P}}\right]$, transformando a eq. $\mathbf{6 . 3 0} \mathrm{em}$ :

$$
\underbrace{\mathbf{\sim}_{\mathrm{o}}\left[\overline{\mathbf{T}}_{\mathrm{u}}\right]}_{\mathbf{H}_{\mathrm{A}}} \underbrace{\mathbf{G}_{\mathrm{O}}\left[\overline{\mathbf{T}}_{\mathrm{P}}\right]}_{\mathbf{U}_{\mathrm{A}}} \underbrace{\mathbf{P}_{\mathrm{A}}}_{\mathbf{G}_{\mathrm{A}}}
$$

\subsubsection{2 - LIGAÇÃO ENTRE REGIÕES DE PLACAS E BARRAS (MEC/MEF)}

Agora, da mesma maneira que analisado para ligação de placas, analisa-se aqui a influência da excentricidade entre os planos neutros das placas e eixo neutro de barras acopladas, que leva a um estado plano de tensão sobre o pavimento.

Originalmente, a equação de equilíbrio das regiões de barras se refere ao eixo neutro de cada barra (origem em $\mathbf{O}$ - fig. 6.21):

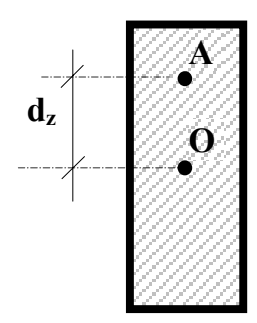

Fig. 6.21- Seção transversal de barra reta

$$
\underset{\sim}{\mathbf{K}_{\mathrm{o}}} \mathbf{U}_{\mathrm{o}}={\underset{\sim}{\mathrm{O}} \mathbf{P}_{\mathrm{O}}}^{\mathbf{F}_{\mathrm{e} 0}}
$$

ou, escrevendo-se na sua forma mais comum, com o segundo membro apenas em termos de forças equivalente: 


$$
\underset{\sim}{\mathbf{K}_{\mathrm{o}} \mathbf{U}_{\mathbf{0}}}=\underset{\sim}{\mathbf{F}_{0}}
$$

São equivalentes, portanto, os sistemas de forças com origens nos nós $\mathbf{A}$ e $\mathbf{O}$, sendo então, válida a relação:

$$
\underset{\sim_{\mathrm{A}}}{\mathbf{F}}=\underset{\sim}{\Lambda} \cdot{\underset{\sim}{\mathbf{F}}}_{\mathbf{O}}
$$

Para se escrever agora, a equação de equilíbrio em relação ao eixo que passa por A a partir O, utiliza-se o PTV, isto é, pode-se afirmar que:

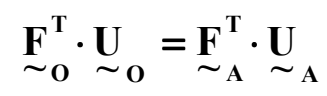

e a partir de eq. 6.51 obter-se

$$
\mathbf{U}_{\mathrm{o}}={\underset{\sim}{\Lambda}}^{\mathrm{T}} \cdot{\underset{\sim}{\mathrm{A}}}_{\mathrm{U}}
$$

Transcrevem-se aqui as componentes da matriz $\underset{\sim}{\Lambda}$ (eq. 6.28), que para um determinado nó i, vale:

$$
\underset{\sim}{\Lambda_{i}}=\left[\begin{array}{cc}
\mathbf{I}_{3} & 0_{3} \\
\sim & \sim \\
\Omega_{3} & \sim \\
\sim & \sim
\end{array}\right]
$$

sendo $\mathbf{I}_{3}, \mathbf{0}_{3}$ e ${\underset{\sim}{i}}_{i}$, respectivamente, as matrizes de ordem três identidade, nula e:

$$
\underset{\sim}{\Omega_{3}}=\left[\begin{array}{ccc}
0 & -d_{z} & d_{y} \\
d_{z} & 0 & -d_{x} \\
-d_{y} & d_{x} & 0
\end{array}\right]_{3}=-\Omega_{3}^{T}
$$


Substituindo-se eqs. 6.50, e 6.53 em 6.51, chega-se a :

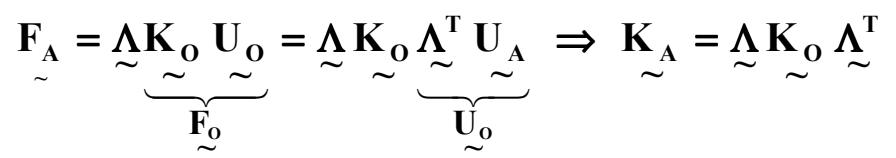

sendo que para uma barra $\overline{\mathbf{i j}}$, a matriz $\underset{\sim}{\Lambda}$ é:

$$
\underline{\sim}=\left[\begin{array}{cc}
\Lambda_{\mathrm{i}} & \mathbf{0}_{6} \\
\underset{\mathbf{0}_{6}}{\Lambda_{\mathrm{j}}} & \sim
\end{array}\right]
$$

Para obter-se a representação para a matriz de transformação do vetor de forças $\underset{\sim}{\text { C sabe-se que: }}$

$$
\underset{\sim}{\mathbf{F}_{0}}=\underset{\sim}{\mathbf{C}_{0}} \mathbf{P}_{\mathbf{O}}
$$

e a partir das eq. 6.51 chega-se a:

$$
\underset{\sim}{\mathbf{C}_{\mathrm{A}}}=\underset{\sim}{\Lambda} \mathbf{C}_{\mathrm{\sim}_{0}} \Lambda_{\sim}^{-1}
$$

E para o vetor de forças externas:

$$
\underset{\sim}{\mathbf{F}_{\mathrm{eA}}}=\underset{\sim}{\Lambda} \mathbf{F}_{\mathrm{eO}}
$$

Supondo-se que haja apenas a excentricidade $\mathbf{d}_{\mathrm{z}}, \quad\left(\mathbf{d}_{\mathrm{x}}=0, \mathbf{d}_{\mathrm{y}}=0\right)$, para um determinado nó ị, a matriz de rigidez terá, originalmente, os componentes: 


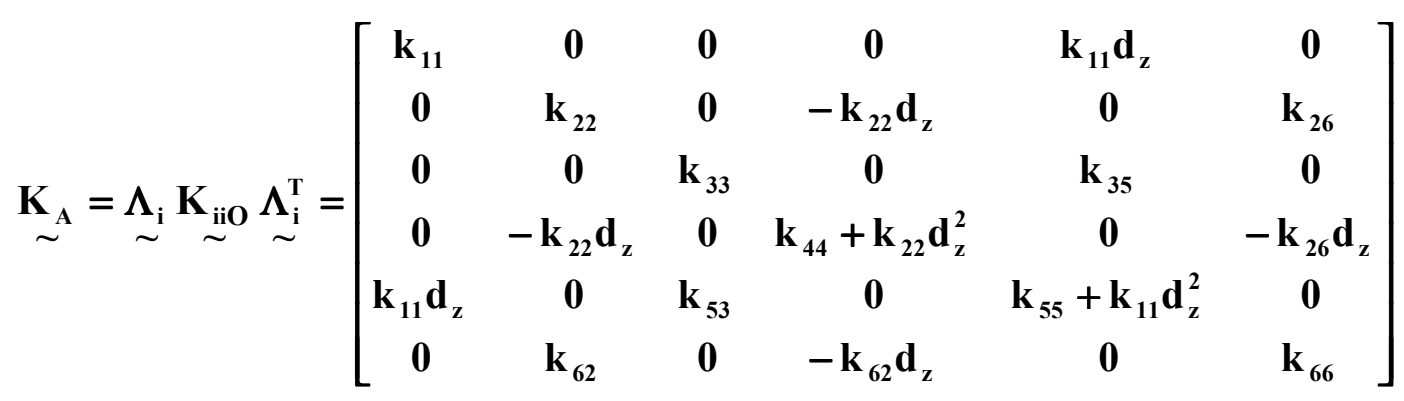

onde $\mathbf{k}_{\mathbf{m j}}$ são componentes da matriz de rigidez da barra $\mathbf{i}$, escrita para um de seus nós.

Neste caso, observe-se o surgimento de componentes nas direções de $\mathbf{u}_{\mathbf{1}}$ e $\mathbf{u}_{\mathbf{2}}$. Após a transformação de $\mathbf{C}_{\mathbf{o}}$, surgem também componentes nas direções de $\mathbf{p}_{\mathbf{1}}$ e $\mathbf{p}_{\mathbf{2}}$. Como o presente estudo elabora a combinação entre sub-domínios através da técnica de sub-regiões, a verificação do equilíbrio e da compatibilidade de deslocamento na região da interface estarão garantido(vide item 6.5).

\subsubsection{3 - EXEMPLOS}

\section{EXEMPLO I:}

Este exemplo é o mesmo apresentado na fig. 6.7a. Desta vez, será considerada a excentricidade vertical $\mathbf{d z}$ do eixo da viga em relação ao plano neutro da placa. Este caso foi também analisado por TANAKA \& BERCIN (1997).

Neste exemplo, o pavimento foi subdividido em duas regiões iguais de placa e uma barra, esta última disposta ao longo da interface entre as placas. O contorno das placas foi subdividido em 12 elementos de contorno, três por lado. A barra foi subdividida em 6 elementos finitos. Na fig. 6.22a estão os dados de geometria e de propriedades físicas do material da viga. Nesta mesma figura encontram-se representados os deslocamentos de uma linha de nós perpendicular à barra, e que se estende de um bordo apoiado da placa ao ponto $\mathbf{A}$. 
Os resultados calculados por TANAKA \& BERCIN (1997) para o nó A, estão indicados nos gráficos (para $\mathbf{x} / \mathbf{a}=\mathbf{0 , 5}$ ). Observe-se que, tanto no caso da viga excêntrica como no da concêntrica, os resultados de deslocamentos em A calculados pelos dois métodos são bastante próximos. Outro fato a se observar é a grande redução no valor dos deslocamentos em geral quando se considera a barra excêntrica:

Tanaka et al. (1997) Presente Trabalho viga concêntrica

$\left(\mathrm{wA}^{*} \mathbf{1 0}^{-3} \mathrm{~cm}\right)$

1,147

1,175 viga excêntrica

$\left(\mathrm{wA}^{*} \mathbf{1 0} \mathbf{0}^{-3} \mathrm{~cm}\right)$

0,314

$\mathbf{0 , 3 8 4}$

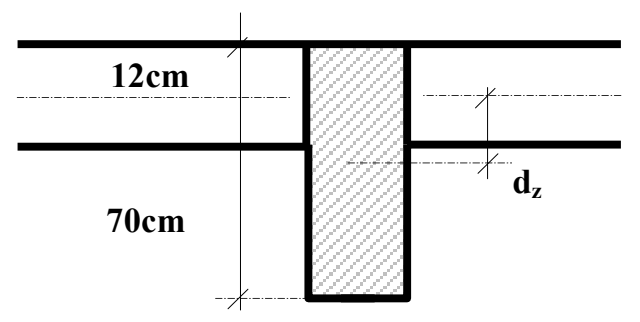

$\mathrm{a}=\mathbf{2 , 0 \mathrm { m }}$

$\mathbf{t}=\mathbf{0 , 1 2} \mathbf{m}$

$v=0,0$

$\mathrm{E}=1,18 \times 10^{7} \mathrm{kN} / \mathrm{m}^{2}$

$\mathrm{q}=\mathbf{0 , 4 5} \mathrm{kN} / \mathrm{m}^{2}$

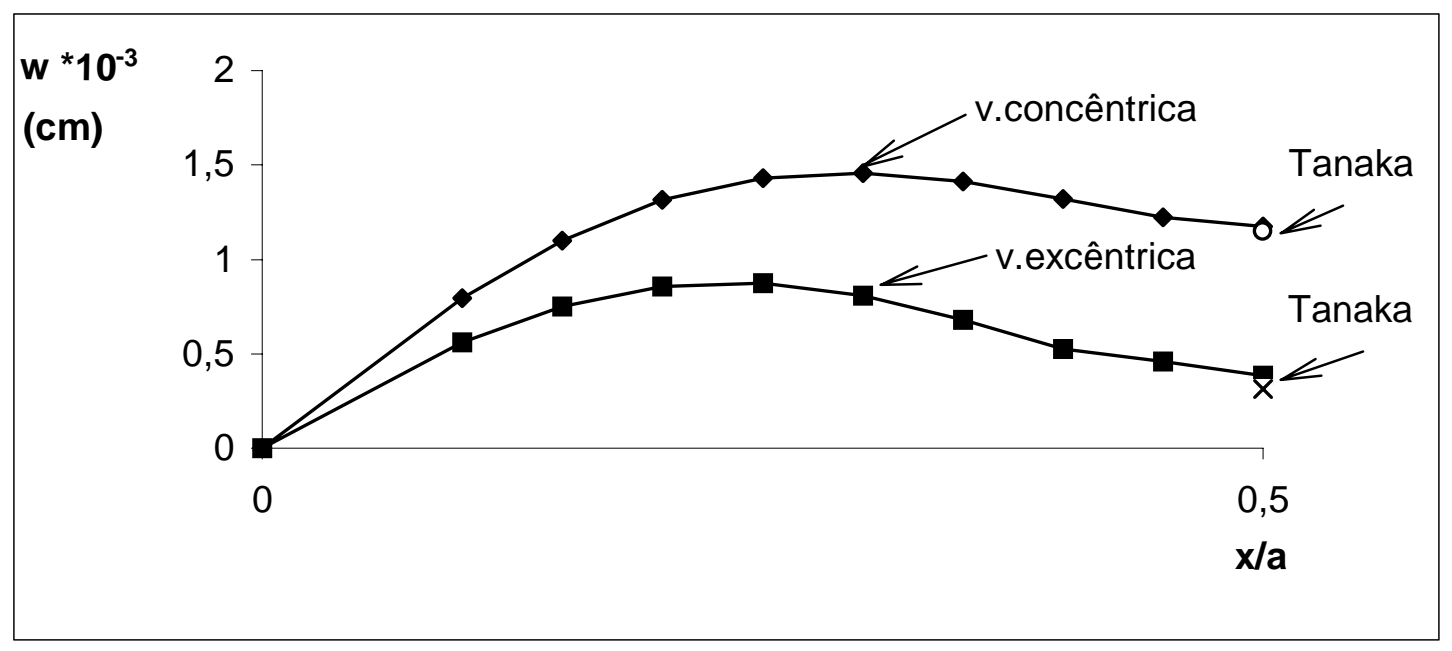

Fig. 6.22a - Exemplo I - Laje da fig. 6.7a com viga concêntrica e excêntrica 
Estes exemplos (figs. 6.22b e 6.22c), onde são investigados os deslocamentos dos nós A, também foram analisados por TANAKA \& BERCIN(1997) e PAIVA(1987), respectivamente, porém apenas para o caso das vigas concêntricas. Nesta tese, calculou-se os deslocamentos dos pontos A dos exemplos, inclusive com a consideração da excentricidade das vigas.

No exemplo II e no caso das vigas concêntricas, TANAKA \& BERCIN(1997) calcularam $\mathbf{w}_{\mathbf{A}}=\mathbf{4 , 1 2} \mathbf{f w}$, sendo $\mathbf{f w}=\mathbf{1 0}^{-4} \mathbf{q} \mathbf{L}^{4} / \mathbf{D}$. Neste trabalho, obteve-se $\mathbf{w}_{\mathrm{A}}=\mathbf{4 , 2 1} \mathrm{fw}$ para as vigas concêntricas e $\mathbf{w}_{\mathrm{A}}=\mathbf{4 , 0 5} \mathrm{fw}$ para as excêntricas.

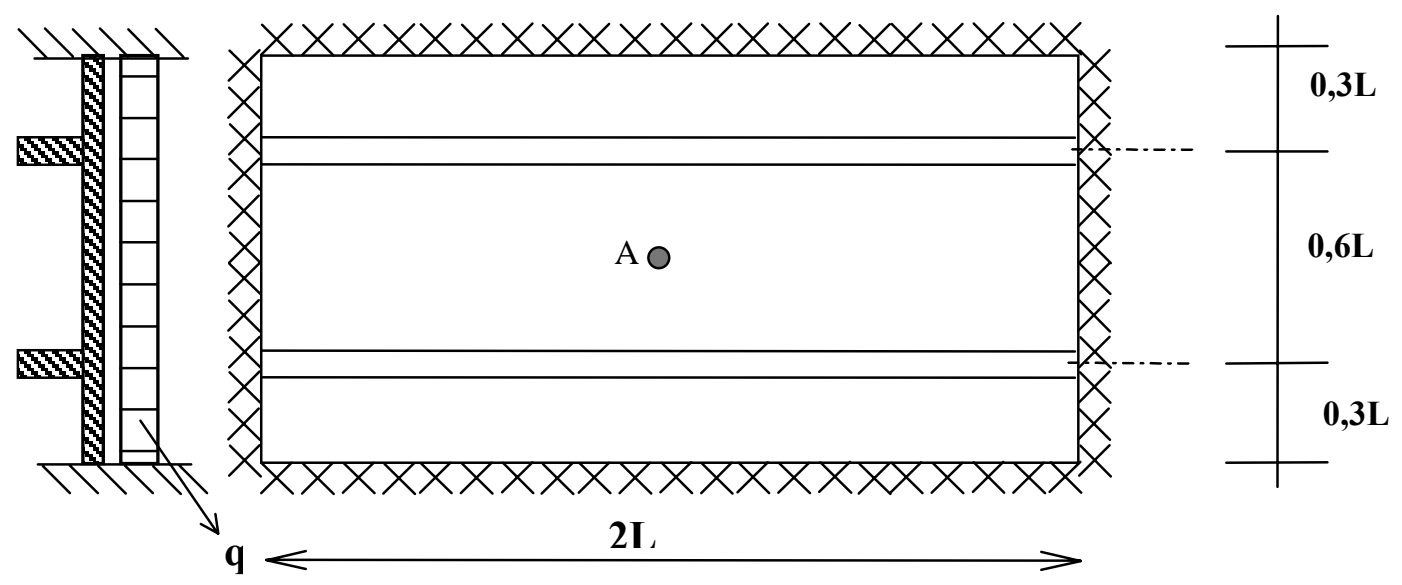

Fig. 6.22b - Exemplo II

No exemplo III, considerando-se agora $f \mathbf{w}=\mathbf{1 0}^{-3} \mathbf{q a}^{\mathbf{4}} / \mathrm{D}$, PAIVA(1987) obteve $\mathbf{w}_{\mathbf{A}}=\mathbf{4 , 7 0 5} \mathbf{f w}$ para as vigas concêntricas. Os resultados calculados pelo algoritmo desenvolvido nesta tese para este exemplo foram $\mathbf{w}_{\mathbf{A}}=\mathbf{4 , 7 0 6} \mathrm{fw}$ para as vigas concêntricas e $\mathbf{w}_{\mathbf{A}}=\mathbf{0 , 3 7 4} \mathbf{f w}$ para as excêntricas. 


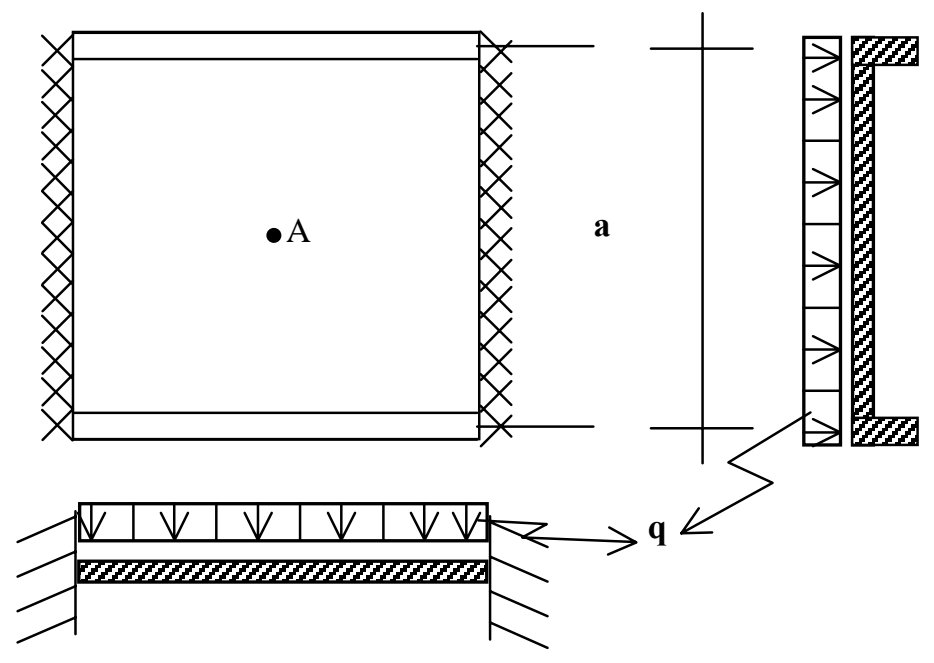

Fig. 6.22a._- Exemplo III

Observa-se que, pelo valor da redução dos deslocamentos quando se considera a excentricidade das vigas, dependendo das características físicas e geométricas do pavimento, o efeito dos enrijecedores pode ser ou não significativo. 


\section{CAPÍTULO 7 - CONDENSAÇÃO ESTÁTICA E ANÁLISE POR SUBESTRUTURAÇÃO}

\section{1 - INTRODUÇÃO}

Num passado muito próximo, as limitações das tecnologias disponíveis em geral para análise de estruturas, estimularam o surgimento de diversas técnicas e métodos que a facilitassem e acelerassem. Os vários métodos de cálculo, como o MDF, o MEF, o MEC, e técnicas de resolução de sistemas, como a eliminação de graus de liberdade internos através da condensação estática, são resultantes do esforço em se incorporar melhorias aos processos de análise de estruturas.

A condensação estática, em linhas gerais, trata-se de uma redução das matrizes de rigidez e de transformação tensão-deformação, concebida como uma extensão da eliminação de Gauss. Conforme dito por WILSON (1974), porém, o método podese estender à redução do número de graus de liberdade do sistema estrutural completo. A análise por subestruturação considera a estrutura total como uma montagem de subestruturas e sobre a qual se pode aplicar a condensação estática. Nos dias de hoje, apesar do desenvolvimento tecnológico, especialmente com relação 
a equipamentos, o uso desta técnica permite, dentre outras coisas, por exemplo, reduzir o número de graus de liberdade do sistema de equações, diminuindo o esforço de avaliação da natureza dos resultados por parte do calculista. Não se deve, também, abandonar uma técnica elaborada para otimizar o processamento e precisão dos resultados, principalmente quando houver associação entre pavimentos de vários níveis. Da forma como neste trabalho os sistemas de equações finais das estruturas são construídos, atendendo à heterogeneidade entre os diversos subdomínios a que representam, seja ela geométrica, constitutiva ou relativa ao método usado na modelagem (MEC ou MEF), pode-se notar a esparsidade entre as sub-matrizes que os compõem (vide capítulos 6 e 7). VENTURINI (1983) comenta a que existem muitos trabalhos desenvolvidos na área de cálculo numérico para resolver este tipo de sistema, como o de CROTTY (1982) que aplica eliminação de Gauss sobre linha

e colunas dos blocos. É possível se estender a este caso os conceitos das técnicas de condensação e sub-estruturação da matriz de rigidez, como foi visto. Portanto, seguir-se-á o mesmo raciocínio para o caso de estruturas compostas por sub-regiões e procedendo-se com os graus de liberdade associados aos nós dos trechos de interfaces como, nestas técnicas, se faz com graus a se eliminar.

\section{2 - SOLUÇÃO DIRETA UTILIZANDO-SE ALGORITMO BASEADO NA ELIMINAÇÃO DE GAUSS}

Através da utilização de exemplo prático, BATHE (1982) apresenta a formulação para a elaboração de algoritmo baseado na eliminação de Gauss para solução de sistemas de equações, representado pelas matrizes de rigidez de barras. Para a pré-resolução do sistema para graus de liberdade internos, comenta o autor, a explicação física é de que a matriz de rigidez resultante é equivalente à matriz de rigidez da barra quando se liberado grau em evidência.

BATHE (1982) também avalia a formulação na forma matricial, como se segue. Suponha-se um sistema formado por barras com graus de liberdade internos a ser suprimidos (representados por vetores e sub-matrizes com sub-índice a ) e com graus de liberdade a serem mantidos na matriz de rigidez reduzida (por sua vez, 
representados pelo sub-índice $\underline{\mathbf{b}}$ ). A equação matricial de equilíbrio (virá do capítulo de $\mathrm{EF}$ ) pode ser escrita de forma particionada como:

$$
\left[\begin{array}{cc}
\mathbf{K}_{\mathrm{aa}} & \mathbf{K}_{\mathrm{ab}} \\
\mathbf{K}_{\mathrm{ba}} & \mathbf{K}_{\mathrm{bb}}
\end{array}\right]\left\{\begin{array}{l}
\mathbf{u}_{\mathrm{a}} \\
\mathbf{u}_{\mathrm{b}}
\end{array}\right\}=\left\{\begin{array}{l}
\mathbf{F}_{\mathrm{a}} \\
\mathbf{F}_{\mathrm{b}}
\end{array}\right\}
$$

Procede-se a eliminação do grau $\mathbf{u}_{\mathrm{a}}$ a partir do primeiro grupo de equações do sistema na forma:

$$
\mathbf{u}_{\mathrm{a}}=\mathbf{K}_{\mathrm{aa}}^{-1}\left(\mathbf{F}_{\mathrm{a}}-\mathbf{K}_{\mathrm{ab}} \mathbf{u}_{\mathrm{b}}\right)
$$

Da substituição de (7.2) na segunda das equações do sistema (7.1), tem-se que:

$$
\underbrace{\left[\mathbf{K}_{\mathrm{bb}}-\mathbf{K}_{\mathrm{ba}}\left(\mathbf{K}_{\mathrm{aa}}^{-1} \mathbf{K}_{\mathrm{ab}}\right)\right]}_{\mathbf{K}^{\prime}} \cdot \mathbf{u}_{\mathrm{b}}=\underbrace{\mathbf{F}_{\mathbf{b}}-\mathbf{K}_{\mathrm{ba}}\left(\mathbf{K}_{\mathrm{aa}}^{-1} \mathbf{F}_{\mathrm{a}}\right)}_{\mathbf{F}^{\prime}}
$$

WILSON (1974) comenta que os termos $\mathbf{K}_{\mathrm{ba}}\left(\mathbf{K}_{\mathrm{aa}}^{-1} \mathbf{K}_{\mathrm{ab}}\right)$ e $\mathbf{K}_{\mathrm{ba}}\left(\mathbf{K}_{\mathrm{aa}}^{-1} \mathbf{F}_{\mathrm{a}}\right)$ da eq.(7.3) representam, respectivamente, a modificação na rigidez da estrutura devido ao alívio dos graus em a e à transferência da força que estava em $\underline{\mathbf{a}}$ para $\underline{\mathbf{b}}$. Note-se que, a matriz de rigidez reduzida [K'] é da ordem da sub-matriz $\left[\mathbf{K}_{\mathrm{bb}}\right]$ e resulta das transformações sofridas pela matriz original. Estas transformações independem das componentes do vetor de forças $\{\mathbf{F}\}$.

O exemplo dado para ilustrar o conceito básico desta técnica utilizou um sistema de equações construído a partir da MEF. Propriedades da matriz de rigidez original, como a simetria e a positividade, são extensíveis às matrizes reduzidas resultantes da eliminação de graus de liberdade, conforme apresentado acima. Podese, portanto, tirar vantagem desta característica quando se trata de economia de armazenamento de dados. 
O mesmo, entretanto, não pode ser dito dos sistemas construídos por formulações mistas, como é o caso do presente trabalho.

A forma matricial como foi apresentado nas eq. (7.1) a (7.3) induz a multiplicações e inversão de matrizes que retira a eficiência do programa computacional. Deve-se, portanto, proceder a condensação estática utilizando-se a eliminação de Gauss seqüencialmente sobre cada grau de liberdade a ser eliminado. E mais: como a matriz de rigidez global é composta pela contribuição de cada elemento individualmente, esta eliminação pode ser iniciada desde a montagem da matriz elementar. Isto nada mais é que a realização de parte da eliminação de Gauss aplicada ao sistema total, porém, no universo do elemento. Muitos autores, como BATHE(1982) e WILSON (1974), apresentam algoritmo para condensação de cada grau de liberdade por vez. Este procedimento reduz a ordem da matriz do sistema final evitando estocagem de dados e diminuindo o esforço computacional. Outra vantagem lembrada por BATHE(1982) diz respeito aos elementos repetitivos e, idênticos, para os quais ele aconselha a criação de bibliotecas.

\section{3 - ANÁliSE POR SUBESTRUTURAÇÃO}

$\mathrm{Na}$ análise por subestruturação, a estrutura total é considerada como uma montagem de subestruturas às quais se pode aplicar a condensação estática. A possibilidade real de existência de elementos ou subestruturas repetitivas facilita o processo. Cada subestrutura é um conjunto de elementos que terá os graus de liberdade internos condensados. O resultado é que, a matriz de rigidez total final é formada, portanto de sub-matrizes condensadas.

Na sub-estruturação, então cada sub-matriz será tratada como macro-elementos, cujos graus de liberdade internos são condensados. Novamente, BATHE (1982) aconselha a criação de bibliotecas para estocar dados relativos a sub-estruturas repetitivas e a definição de níveis de sub-estruturação, para se aumentar a eficiência da análise que se utiliza desta técnica. 
Esta é uma aplicação eficaz da eliminação de Gauss.

Pode-se, portanto, seguir este mesmo raciocínio para o caso de estruturas compostas por sub-regiões e procedendo-se com os graus de liberdade associados aos nós dos trechos de interfaces como aqui se fez com os nós a serem eliminados.

\section{4-MÉTOdo DA CONDENSAÇÃo ESTÁTICA PARA O SISTEMA MODELADO PELA COMBINAÇÃO DO MEC COM O MEF}

A elaboração do processo se dará na forma matricial para ilustração dos conceitos básicos da formulação aplicada ao sistema de equações dele resultante da combinação de sub-regiões modeladas pelo MEC e/ou pelo MEF. A eliminação no universo da sub-região seguirá as técnicas expostas nos itens anteriores deste capítulo.

Suponha-se o exemplo de duas sub-regiões de placas acopladas ao longo de uma interface. Conforme visto no capítulo 6, a equação matricial do sistema final pode ser escrita de forma particionada como:

$$
\left[\begin{array}{cccc}
A_{1} & 0 & H_{12} & G_{12} \\
0 & A_{2} & H_{21} & -G_{21}
\end{array}\right]\left\{\begin{array}{l}
X_{1} \\
X_{2} \\
U_{21} \\
P_{21}
\end{array}\right\}=\left\{\begin{array}{l}
B_{1} \\
B_{2}
\end{array}\right\}
$$

tendo já sido consideradas as condições de contorno, equilíbrio e compatibilidade. Lembrando-se que, para análise de placas, o sistema de equações é composto de pares de equações por nó (uma para $\mathbf{w}$ e outra para $\mathbf{w}_{\mathbf{A}}$ ), considere-se, agora, estas equações agrupadas em sub-matrizes, de forma a se reescrever a eq.(7.4) como: 


$$
\left[\begin{array}{cccc}
\mathbf{A}_{1}^{\mathrm{w}} & \mathbf{0} & \mathbf{H}_{12}^{\mathrm{w}} & \mathbf{G}_{12}^{\mathrm{w}} \\
\mathbf{A}_{1}^{\mathrm{w}_{\mathrm{A}}} & \mathbf{0} & \mathbf{H}_{12}^{\mathrm{w}_{\mathrm{A}}} & \mathbf{G}_{12}^{\mathrm{w}_{\mathrm{A}}} \\
\mathbf{0} & \mathbf{A}_{2}^{\mathrm{w}} & \mathbf{H}_{21}^{\mathrm{w}} & -\mathbf{G}_{21}^{\mathrm{w}} \\
\mathbf{0} & \mathbf{A}_{2}^{\mathrm{w}_{\mathrm{A}}} & \mathbf{H}_{21}^{\mathrm{w}_{\mathrm{A}}} & -\mathbf{G}_{21}^{\mathrm{w}_{\mathrm{A}}}
\end{array}\right]\left\{\begin{array}{l}
\mathbf{X}_{1} \\
\mathbf{X}_{2} \\
\mathbf{U}_{21} \\
\mathbf{P}_{21}
\end{array}\right\}=\left\{\begin{array}{c}
\mathbf{B}_{1}^{\mathrm{w}} \\
\mathbf{B}_{1}^{\mathrm{w}_{\mathrm{A}}} \\
\mathbf{B}_{2}^{\mathrm{w}} \\
\mathbf{B}_{2}^{\mathrm{w}_{\mathrm{A}}}
\end{array}\right\}
$$

Conforme já comentado, os graus de liberdade associados aos nós dos trechos de interface serão condensados, permitindo-se construir um sistema que envolverá apenas $\mathbf{X}_{1}$ e $\mathbf{X}_{\mathbf{2}}$. Viu-se em 7.2, porém, que deve-se evitar a série de inversões e multiplicação de matrizes que este procedimento envolveria. Daí, conclui-se que, a eliminação dos graus de liberdade associados aos pontos de interface, deverá ser feita individualmente a montagem das matrizes de influência de cada sub-região estiver sendo calculada, antes mesmo da montagem do sistema total, ilustrado em eq.(7.5).

De acordo com a sugestão de WILSON (1974), então, suponha-se que todos os graus a serem eliminados, num total de NGLE, estejam agrupados em seqüência, apenas para facilitar o entendimento. No total, o sistema tem NGL graus de liberdade. Então, partindo-se do mesmo princípio do item 7.2, para $\mathbf{n}$ variando de $\mathbf{1}$ a NGLE, seguem-se os passos sucessivos:

1)Elimina-se um grau $\mathbf{u}_{\mathbf{n}}$ com as expressões:

$$
\begin{aligned}
& \mathbf{C}_{\mathrm{n}}=\mathbf{F}_{\mathbf{n}}^{\prime} \mathbf{K}_{\mathrm{nn}}^{\prime} \\
& \mathbf{T}_{\mathrm{nj}} \mathbf{u}_{\mathrm{j}}=\mathbf{K}_{\mathbf{n j}}^{\prime} / \mathbf{K}_{\mathbf{n n}}^{\prime} \\
& \mathbf{u}_{\mathrm{n}}=\mathbf{C}_{\mathbf{n}}-\sum_{\mathbf{j}=\mathbf{n}+\mathbf{1}}^{\mathrm{NGL}} \mathbf{T}_{\mathbf{n j}} \mathbf{u}_{\mathbf{j}}
\end{aligned}
$$

2)O valor de $\mathbf{u}_{\mathbf{n}}$ é substituído nas (NGL-n) equações do sistema restantes, isto é, fazendo-se $\mathbf{i}, \mathbf{j}=\mathbf{n}+\mathbf{1}$, NGL nas seguintes expressões: 


$$
\begin{aligned}
& \mathbf{K}_{\mathrm{ij}}^{\prime \prime}=\mathbf{K}_{\mathrm{ij}}^{\prime}-\mathbf{K}_{\mathrm{in}}^{\prime} \mathbf{T}_{\mathrm{nj}} \\
& \mathbf{P}_{\mathrm{i}}^{\prime \prime} \mathbf{u}_{\mathrm{j}}=\mathbf{P}_{\mathrm{i}}^{\prime}-\mathbf{K}_{\mathrm{in}}^{\prime} \mathbf{C}_{\mathbf{n}}
\end{aligned}
$$

WILSON (1974) demonstra este procedimento em forma de algoritmo, para um universo de um determinado exemplo.

Como foi dito, porém, existem programas e bibliotecas de pacotes matemáticos disponíveis que resolvem sistemas de equações levando em conta a esparcidade das matrizes. A intenção neste capítulo foi a familiarização com processo, pois acredita-se que pode-se tirar vantagens das características do sistema de equações resultante das técnicas utilizadas neste trabalho. Providências para lançar mão destes benefícios devem ser tomadas desde a definição divisão do domínio em estudo em sub-regiões, discretização do contorno e preparo da entrada de dados.

Com base nos aspectos aqui abordados e nas características do sistema de equações resultante, elaborou-se um algoritmo para possibilitar a resolução do sistema do algoritmo desenvolvido neste estudo através da biblioteca matemática DLSLXG do FORTRAN POWERSTATION 4.0 (1994-1995). Esta rotina resolve sistemas esparsos de equações lineares algébricas por eliminação de Gauss. A linha de comando para a chamada desta rotina é:

\section{CALL DLSLXG (N, NZ, A, IROW, JCOL, B, X)}

cujos principais argumentos de entrada são:

$N$ - Números de equações do sistema (Input)

$\mathrm{NZ}$ - Número de coeficientes não-nulos do sistema (Input)

$\boldsymbol{A}$ - Vetor de tamanho NZ contendo os coeficientes não-nulos (Input)

IROW - Vetor de tamanho NZ contendo os números das linhas dos coeficientes não-nulos em A. (Input)

JCOL - Vetor de tamanho NZ contendo os números das colunas dos coeficientes não-nulos em A (Input)

$\boldsymbol{B}$ - $\quad$ Vetor de termos independentes de tamanho N (Input)

$\boldsymbol{X}-\quad$ Vetor de tamanho N a solução do sistema (Output) 


\section{CAPÍTULO 8 - CONCLUSÕES DO TRABALHO}

Conforme mencionado, a idéia deste trabalho foi contribuir com a gama de trabalhos desenvolvidos para a análise de placas com enrijecedores através da combinação entre os métodos dos elementos de contorno e finitos. A principal contribuição se deve à consideração da excentricidade do eixo dos enrijecedores com relação ao nível do acoplamento, somando-se à flexão da estrutura, um estado plano de tensões.

Elaborou-se um algoritmo para descrever o comportamento estrutural das placas através do MEC, com base na teoria de Kirchhoff. Também pelo MEC equacionou-se o elemento de chapas para efeito da combinação do elemento de superfície com barras excêntricas, quando se considera a excentricidade do eixo neutro destas em relação à superfície neutra das placas. Finalmente, as barras foram modeladas pelo MEF e abordam-se neste trabalho, apenas aspectos relevantes à adaptação do sistema gerado para efeito de acoplamento com regiões modeladas pelo MEC. A breve explanação se justifica por se tratar de assunto já bastante difundido.

A técnica escolhida para proceder a combinação foi a das sub-regiões, que estabelece condições de equilíbrio e compatibilidade de deslocamentos na linha de 
interface de união entre estes subdomínios. Vários aspectos e particularidades desta técnica foram estudados no intuito de se atingir muitas das possibilidades de variações de ligações. Exemplos numéricos foram apresentados com resultados satisfatórios, comprovando a total implementação da rotina gerada ao programa principal. Estes exemplos mostraram, também, as discrepâncias nos resultados quando a subdivisão do domínio em estudo é feita inadequada e desnecessariamente. Mais adiante se soma à praticidade do uso desta técnica a otimização do sistema conferida pela técnica da condensação estática com base na eliminação de Gauss. Sua aplicação é facilitada pela primeira, na resolução do sistema de equações gerado. Por saber-se não haver grandes ganhos com relação à velocidade de processamento (o mesmo não se deve dizer com relação aos ganhos em melhoria de resultados), não houve comparação de resultados, tendo sido a biblioteca $\boldsymbol{D L S L X G}$ do FORTRAN POWERSTATION 4.0 (1994-1995) adotada como rotina de resolução de sistemas do programa.

Em seguida, expõem-se aspectos da ligação entre barras e placas assim definidas, para elaborar a consideração do efeito de membrana. Em primeiro lugar, soluções adotadas para acoplamento de barras e chapas com coincidência de eixos é apresentada. Resultados de exemplos numéricos comprovam a validade do algoritmo sugerido. Depois disto, idealiza-se o modelo para a consideração do efeito de membrana no plano da placa. Utiliza-se o PTV ou a simples observação do equilíbrio da força excêntrica e compatibilização dos deslocamentos na região da ligação. Novamente, os resultados de exemplo numérico demonstram o modelo deduzido, atentando-se para a variação nos resultados quando se considera a excentricidade das barras em relação ao plano neutro das placas.

Com base no que aqui se estudou, um proprietário de algoritmo de análise de placas e chapas através do MEC e de barras pelo MEF, pode elaborar uma rotina de acoplamento entre as diversas sub-regiões sem necessariamente precisar modificar as suas formulações básicas. Deve estar atento, porém, à criteriosa definição destes subdomínios a fim de evitar a inclusão desnecessária e muitas vezes inexistente de descontinuidade nas regiões das interfaces. Uma pré-análise das condições físicas e 
geométricas do pavimento também ajuda na interpretação dos resultados dos deslocamentos dos nós de placas enrijecidas. 


\section{REFERÊNCIAS BIBLIOGRÁFICAS}

ALIABADI, M. H., \& ROOKE, D.P. Numerical fracture mechanics: solid mechanics and its applications, Southampton-UK, 1992, CMP and Kluwer Academic Publisher.

ARAÚJO, F. C., MANSUR, W.S, NISHIKOWA, L.K., Determination of 3D time domain responses in latared media by using coupled BE/FE process. XX Boudary Elements, 1998, CMP.

BATHE, K. J. Finite element procedures in enginnering analysis. Englewood Cliffs, Prentice-Hall, 1982.

BEER (1986) Implementation of combined boundary element-finite element analysis with applications in geomechanics, p. 191-225, Developments in boundary elements method-4, chapter 4, Ed. P.K. Banerjee, J.O. Watson. Elsevier Appl. Sci. Publishers.

BÉZINE. G. A boundary integral equation method for plate flexure with conditions inside the domain. Int. J. Num. Meth. Eng., v.17, p.1647-1657. 1981.

BREBBIA \& GEORGIOU (1979) Combination of boundary and finite elements in elastostatics, 212-220, Appl. Math. Modelling, v.3, n.3. June.

BREBBIA, C. A. \& DOMINGUES, J. . Boundary Elements - An introductory course. Southampton, CMP, 1989.

CALDERÓN, E.T. \& VENTURINI, W.S.(1997) Analysis of plates in bending resting on 3-dimensional half space using BEM -Boundary Elements XIX Conference in BEM, Italy, 1997, p. 245-254. 
CHAUDOUET-MIRANDA, A. \& CRISTESCU (1993) - The Castor system-why not use FEM and BEM- Industrial applications of the Boundary Element Method - Southampton, UK, 1993.

CHAVES, E.W. V. Análise de placas com variação de espessura, através do método dos elementos finitos. São Carlos, 1997, Dissertação de Mestrado - Escola de Engenharia de São Carlos, USP.

CHAVES, E.W.V , FERNANDES, G.R., VENTURINI, W . S. (1999)

CHUEIRI(1994) Formulação do MEC para análise elastoplástica de placas. Tese de doutoramento - Escola de Engenharia de São Carlos, USP.

CODA, H. B. Análise tridimensional transiente de estruturas pela combinação entre o MEC e o MEF. São Carlos, 1993, Tese de doutoramento - Escola de Engenharia de São Carlos, USP.

CODA, H. B. \& VENTURINI, W.S. BEM/FEM Non-linear model applied to transient Analysis with viscous damping, J. ofBraz. Soc. Mechanical Science, v. XXI, n. 3, 999, p. 519-536.

CODA, H. B. \& VENTURINI, W.S., KOMATSU, J.S>, Coupling for the continuum media-frame structure interaction, The International Journal of BEM Communications, 1997 , p. 73-78.

CODA, VENTURINI, ALIABADI (1997) A simple coupling of 2D BEM and FEM bar model applied to mass matrix elastodynamic analysis, 137-146, Boundary Elements XIX, Rome, September. Section 3: Structural Dynamics.

FERNANDES, G. R. O método dos elementos de contorno aplicado à análise nãolinear de placas. São Carlos, 1998, Dissertação de Mestrado - Escola de Engenharia de São Carlos, USP. 
FRANGI, A. \& GIUGGIANI, M. Boundary element analisys of Kirchhoff plates with direct evaluation of hypersingular integrals. IJNME, (accepted for publication), v. 46, p. 1845-1863, Aug., 1999.

GUO-SHU, S.; MUKERJEE,S. Boundary element analysis of bending plates of arbitrary shape with general boundary conditions. Eng. Analysis, v.3, p. 36-44, 1986. "

HARTMANN, F. Static analysis of plates. Boundary Element Analysis of plates,. 1991. Springer-Verlag.

HARTMANN, F.; ZOTEMANTEL, R. The direct boundary element method in plate bending. Int. J. Num. Meth. Eng., v.23, p.2049-2069. 1986.

KARAM, V. J. \& TELLES, J. C. F. On boundary elements for Reissner's plate theory. Engineering Analysis, 1988, v. 5, n. 1,Computatinal Mechanics Publications.

MESSAFER \& COATES (1989) An application of FEM/BEM coupling to foundation analysis, 211-223, XI International Conference on BEM-Advances in Boundary Elements-Stress Analysis, Cambridge, USA, v.3, proceedings. Ed. C.A. Brebbia, J. J. Connor. Springer-Verlag/Computational Mechanics Publications.

OLIVEIRA NETO, L. Uma formulação do método dos elementos de contorno com três parâmetros em deslocamentos para placas aplicada a problemas de engenharia estrutural. São Carlos, 1998, Tese de doutoramento - Escola de Engenharia de São Carlos, USP.

PAIVA , J.B. \& VENTURINI, W.S., Alternative technique for the solution of plate bending problems using the BEM. Advances in Engineering Software, 14, 1992, p. 265-271. 
PAIVA , J.B. \& VENTURINI, W.S., Analysis of building structures considering plate-beam-column interactions, Boundary Element Techniques - Applications in Stress Analysis and Heat Transfer, CMP, 1987.

PAIVA, J.B. \& VENTURINI, W.S., Boundary element algorithm for building slabs analysis, BETECH 85, nov., 1985

PAIVA, J. B. Boundary element formulation of building slabs. Engineering Analysis with Boundary Elements, 17, 1996, p. 105-110.

PAIVA, J.B. Formulação do método dos elementos de contorno para a flexão de placas e suas aplicações em engenharia de estruturas. São Carlos, 1987. Tese (Doutorado) - Escola de Engenharia de São Carlos, USP.

PAIVA, J.B.; VENTURINI, W.S. Análise de estruturas em laje cogumelo pelo método dos elementos de contorno. In: COLLÓQUIA 1987, Porto Alegre, 29 jun.-3 jul., 1987. Anais. Porto Alegre, CPGEC-UFRGS, 1987. v.4, p.115123.

PALERMO JR, L. Análise de peças de seção delgada como associação de placas pelo MEC. São Carlos, 1989, Tese de doutoramento - Escola de Engenharia de São Carlos, USP.

ROCHA, J. A. L. Contribuição para a teoria termodinamicamente consistente da fratura. São Carlos, 1999, Tese de doutoramento - Escola de Engenharia de São Carlos, USP.

SILVA, N.A. Análise de pavimentos de edifício utilizando uma formulação do MEC baseada na teoria de Reissner. São Carlos, 1996, Tese de doutoramento (aguardando defesa) - Escola de Engenharia de São Carlos, USP. 
TANAKA \& BERCIN (1997), A boundary element method applied to the elastic bending problem of stiffened plates, 203-212, Boundary Elements XIX, Rome, September. Section 5: Plate bending.

TANG, W. Transforming domain integrals in BEM - Lecture Notes in Engineering, 1988, UK, Springer Verlag.

TIMOSHENKO, S. Theory of plates and shells, 1959, McGraw-Hill.

UMETANI, S-I. Adaptive Boundary Element Method on Elastostatics - Topics in Engineering, v .5, Computational Mechanics Publications, UK, 1988.

VENTURINI, W.S., \& PAIVA, J.B., Plate bending analysis by the boundary element method considering zoned thickness domain, Software for Engineering Workstation, oct., 1988, CMP, v. 4.

VENTURINI, W.S., Um estudo do MEC e suas aplicações em problemas de engenharia, Tese de Livre-Docência, São Carlos, 1988.

VENTURINI, W.S., Further development of boundary element formulation for zoned domain problems, 251-267, XI International Conference on BEMAdvances in Boundary Elements-Stress Analysis, Cambridge, USA, v.3, proceedings. Ed. C.A. Brebbia, J. J. Connor. Springer-Verlag/Computational Mechanics Publications. 1989.

WEARING \& BETTAHAR, A boundary element subregioning scheme for plat bending analysis, 293-302, Boundary element method XVI, Southampton, 1215 July, 1994.

WILSON, E. L. The static condensation algorithm. International Journal for Numerical Methods in Engineering, v. 8, n. 1, p. 198-203, 1974. 
ZIENKIEWICZ, O. C. The finite method in enginnering science, 1971, McGraw Hill, England. 